\title{
ON THE DEVELOPMENT OF PHOTOACOUSTIC IMAGING BIOMARKERS FOR CANCER TREATMENT MONITORING
}

\author{
by \\ Eno Hysi \\ B.Sc., Ryerson University, 2010 \\ M.Sc., Ryerson University, 2012 \\ A thesis \\ presented to Ryerson University \\ in partial fulfilment of the \\ requirements for the degree of \\ Doctor of Philosophy \\ in the program of \\ Biomedical Physics
}

Toronto, Ontario, Canada, 2020

(c) Eno Hysi, 2020 


\section{AUTHOR'S DECLARATION FOR ELECTRONIC SUBMISSION OF A DISSERTATION}

I hereby declare that I am the sole author of this dissertation. This is a true copy of the dissertation, including any required final revisions, as accepted by my examiners.

I authorize Ryerson University to lend this dissertation to other institutions or individuals for the purpose of scholarly research.

I further authorize Ryerson University to reproduce this dissertation by photocopying or by other means, in total or in part, at the request of other institutions or individuals for the purpose of scholarly research.

I understand that my dissertation may be made electronically available to the public.

\section{ENO HYSI}




\title{
ABSTRACT
}

\section{On the development of photoacoustic imaging biomarkers of cancer treatment response}

\author{
Eno Hysi \\ Doctor of Philosophy, Biomedical Physics \\ Department of Physics, Ryerson University, 2020
}

This dissertation describes the development of functional and structural photoacoustic (PA) imaging biomarkers that can be used to monitor cancer treatment response and potentially predict treatment outcome. An imaging method that can indicate individualized treatment success could improve therapeutic outcome. Assessing the effectiveness of therapies as early as possible may spare the patient from unnecessary treatments and save precious clinical resources. In order for PA imaging to enter mainstream radiology and become a treatment monitoring tool, rigorous development of biomarkers that are easy-to-use and representative of the treatment-induced changes in the tumor microenvironment are needed. In this work, I have developed imaging biomarkers that rely on the analysis of the radiofrequency signals in acoustic resolution PA imaging. Specifically, I show through simulations and experiments that biomarkers sensitive to the size, number density and spacing of tumor blood vessels can be extracted through time and frequency domain analysis of PA signals. This information is encoded in the speckle that forms during diffuse optical illumination, which was previously thought to be noise. Moreover, I demonstrate that PA imaging can detect the response of a thermosensitive liposome by measuring a $>10 \%$ drop in the oxygenation of the tumor as early as 30 minutes post-treatment. This change in oxygenation is due to vascular disruption, a phenomenon that can be detected through frequency analysis of the PA signals. The spectral slope parameter decreases by as much as $73 \%$ in 2 hours post-treatment and can be used to differentiate alongside the oxygenation biomarker between responders and non-responders. Lastly, I demonstrate that these PA imaging biomarkers correlate well with the histologically measured biophysical changes of two novel, bubble-based cancer treatments. In this dissertation, PA imaging biomarkers for cancer treatment monitoring are developed, advancing the modality towards clinical translation. 


\section{ACKNOWLEDGMENTS}

As I try and remember the countless people that have assisted me throughout my doctorate, I will start with my supervisor, Prof. Michael Kolios. I highly doubt there is going to be another scientist that I meet in my lifetime that I will want to emulate more than him. Mike Kolios first left an impression on me when I was as a young, first-year undergraduate physics student and suffice it to say, that impression reverberates to this day, 13 years later. During this time, I have had the privilege of witnessing three versions of Mike: the first-year physics prof, the Canada Research Chair and the Associate Dean of Science that somehow has time to supervise a research group of $25+$ people. In every single version, Mike Kolios is the epitome of the ideal teacher, mentor and supervisor. An exemplary scientist addicted to physics and espressos alike, his passion for research is utterly contagious and has played a significant role in my personal development as a scientist. I thank him deeply for giving me immense academic freedom in pursuing my interests, even though they did not always align with my thesis. I feel very fortunate to have had the opportunity to be involved with so many different projects and receive tremendous support throughout every single one of them. I struggle to remember a single meeting with Mike where I don't come out feeling full of energy, optimism and inspired to do better science. In my ongoing plight to present Mike with CCC writing, I learned how to communicate my science through his detailed feedback: I will always be very grateful for that. I feel very fortunate to have trained under a supportive mentor whose biggest priority is always the success of his trainees. My own journey is evidence of that dedication, for which I am immensely indebted. I hope to one day share what Mike Kolios has taught me with others, for that is the most suitable way I can think of expressing my unwavering admiration for a fantastic mentor...

I have been very fortunate to receive generous support from a great number of individuals. I'd like to start with acknowledging my supervisory committee members Drs. Raffi Karshafian, Yuan Xu and Gregory Czarnota. I thank all of you for always challenging me. I particularly enjoyed having two first-authors from one of the most crucial papers of my $\mathrm{PhD}$ as part of my committee. Through their open-ended questions I learned the importance of rigor and scientific skepticism which propelled this project forward. I would also like to thank Prof. Xueding Wang and Dr. Dafna Sussman for being part of my examination committee. 
Ever since I was an unexperienced undergraduate research assistant, I have been very privileged to have access to dedicated postdoctoral fellows. I particularly would like to highlight Dr. Lauren Wirtzfeld. Her endless patience with my obscure coding questions at 3 a.m. and her boundless ultrasound knowledge taught me a great deal. She remains one of the most resourceful scientists I had the pleasure of interacting with and I am thankful I got to learn from her. Special thanks also go to Dr. Tae-Hoon Bok. Although not a part of my thesis, our work on aggregation carried out during my $\mathrm{PhD}$ remains one of my proudest achievements. I cherish our scientific discussions and late experiments during his "lab vacations" from the twins. Dr. Ratan Saha also deserves to be recognized for his numerous discussions over the years on photoacoustic speckle. I learned the intricacies of theoretical simulations through him.

Our collaborators at the Ontario Institute for Cancer Research (now at The University of British Columbia) played a crucial role in starting this project. I gratefully acknowledge Dr. Jon May (now at the University of Southampton), the most talented chemist I know for agreeing to teach me all about thermosensitive liposomes. I learned a great deal from his meticulous experiments, and I am very grateful that we spent as many late nights as we did to see if HaT-DOX and PA would yield anything. I remain very appreciative to the generous support of Dr. Shyh-Dar Li in making this project possible by granting us access to many resources, including the very able Elijus Undzys. This thesis would have looked very different without their involvement. I thank Prof. Agata Exner from Case Western Reserve University immensely for bringing her "nanobubble trouble" north of the $49^{\text {th }}$ parallel: it significantly shaped this work. I am very glad that I got to work with her contrast agents that proved to be very good anticancer vehicles. I am also very indebted to the support of our long-time collaborators at Sunnybrook Health Sciences Centre in Dr. Czarnota's laboratory. Special thanks belong to Anoja Giles and Dr. Azza Al-Mahrouki for always finding time to fit me in their insanely busy schedules. Azza and Anoja's patience with my never-ending requests played an integral part in the success of the experiments. I am also thankful for the technical support I received from FujiFilm VisualSonics, particularly on Sunnybrook experiment days.

This work would have simply not been possible without the immediate impact of several individuals from the Kolios lab which served as a catalyst for lasting friendships. Moe Fadhel played an integral part in the development of the theoretical formulation of photoacoustic speckle 
and assistance with in-vivo nanobubble experiments. I am very appreciative of the fact that he always made time to discuss just about everything that was on my laptop screen and never turn down lunch invites. Dr. Mike Moore's contributions are also gratefully acknowledged, as is his belief in my short-lived wedding emceeing career. I am very thankful for Yan Wang's assistance with the nanobubble formulations and I fully enjoyed learning from her vast resourcefulness in the lab. Joseph Sebastian's assistance with histological analysis is greatly appreciated as is his desire to always talk about academia. I thank Dr. Eric Strohm and Jason Zalev for their help on theoretical modeling. Arthur Worthington and Elizabeth Berndl are also gratefully acknowledged for always being invaluable resources in the lab throughout the years. I have enjoyed interacting with many other members of the Kolios lab (past and present) who have created a stimulating and collegial learning environment. Thank you to all of you for making these years memorable. I also would like to give special thanks to my international colleagues Drs. Sophinese Iskander-Rizk, Joanna Brunker and Bisi Bell for making the annual SPIE and IEEE meetings simulating and fun.

As a longtime Ryersonian, I have been fortunate to encounter many mentors in my [too] long tenure here. Within the Department of Physics, I would like to acknowledge the support I have received from Profs. Pedro Goldman, Ana Pejović-Milić, Margaret Bucky and Dr. Carl Kumaradas throughout the years. I am also extremely appreciative of the endless assistance I have received from Matt Forrest, Graham Pearson, Kevin Liu and Sophia Finos. At YSGS, I encountered an amazing team of dedicated individuals that have played a crucial role in my journey as a grad student. I would like to acknowledge the support of the former Dean Dr. Jennifer Mactavish, current Dean Dr. Cory Searcy as well as the Dr. Samantha Wehbi and Leslie Mutic.

Finally, I am very fortunate to have access to a supportive family that has created the perfect ecosystem for me to be able to embark on a long journey. They are my source of strength and inspiration and I owe everything I have ever accomplished to their never-ending love and belief in me. Through everything I attempted, all I ever wanted was reward their sacrifices: I sincerely hope that I succeeded... 
This thesis is dedicated to Lady Gabs 


\section{TABLE OF CONTENTS}

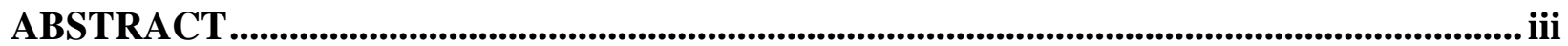

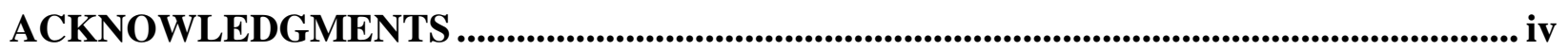

LIST OF TABLES ..........................................................................................................

LIST OF FIGURES .................................................................................................................. xiii

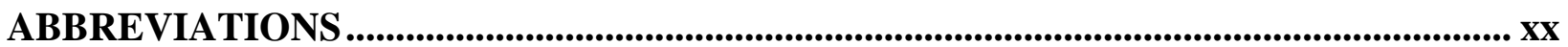

LIST OF SYMBOLS ........................................................................................................... $\times$

CO-AUTHORSHIP STATEMENT .................................................................................. xxii

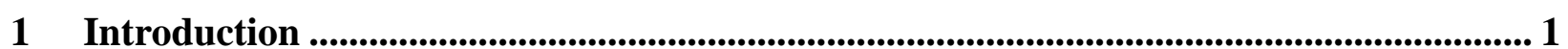

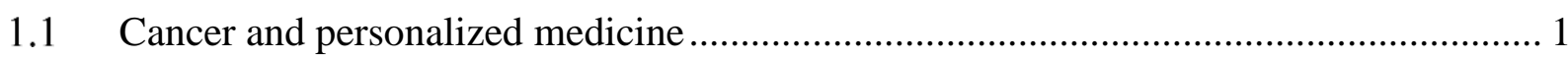

1.2 Personalized treatments require personalized imaging ................................................. 2

1.3 Current imaging standards of treatment monitoring ................................................... 2

1.4 Biomarkers of cancer treatment response ............................................................... 3

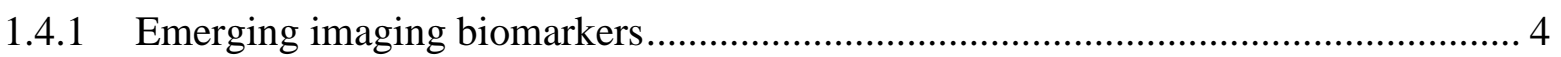

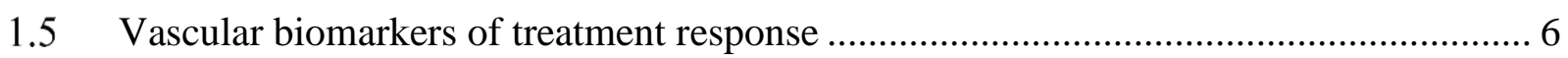

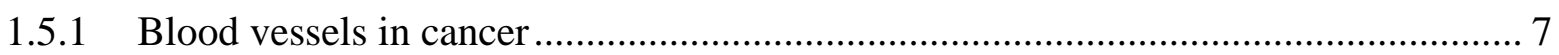

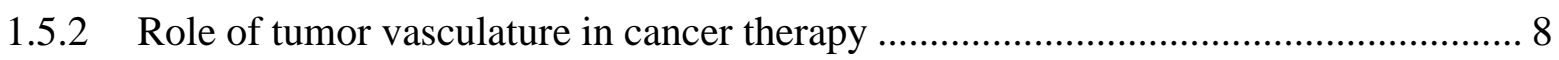

1.6 Photoacoustic imaging and its potential................................................................... 10

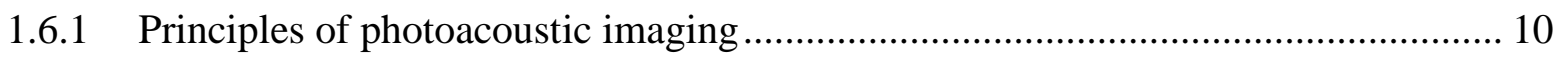

1.6.2 Configurations and applications of PA imaging .................................................... 12

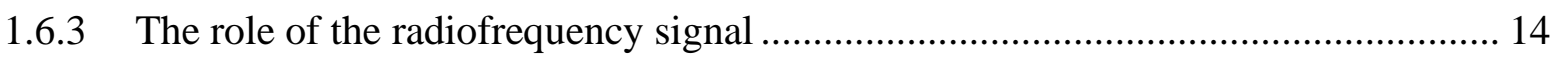

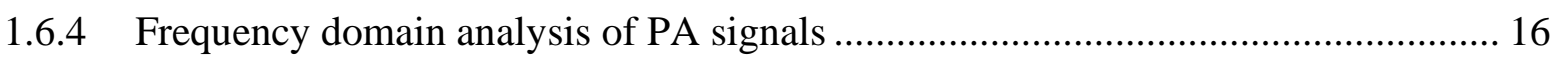

1.6.5 Can PA imaging contribute to treatment monitoring? ............................................. 17

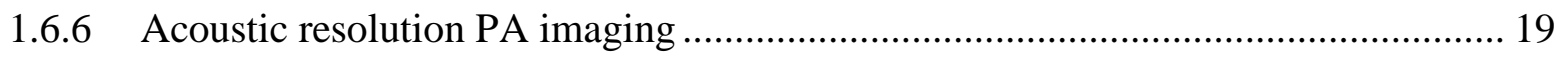

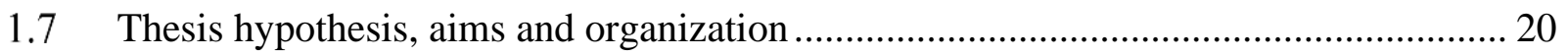

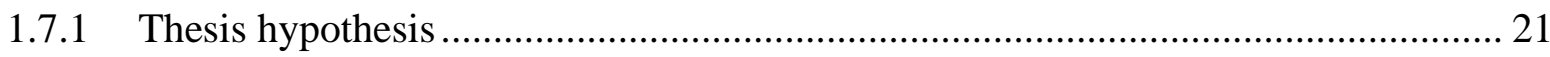

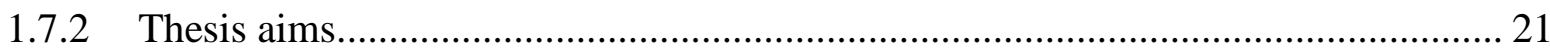

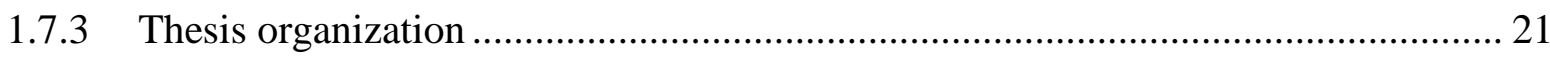

2 Insights into photoacoustic speckle and applications in tumor characterization ........... 24

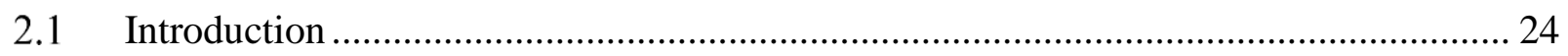

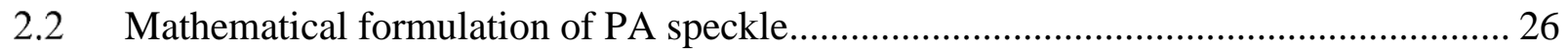

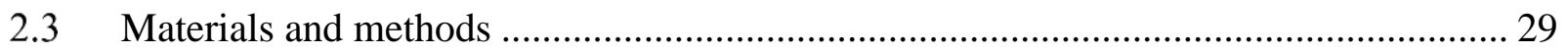

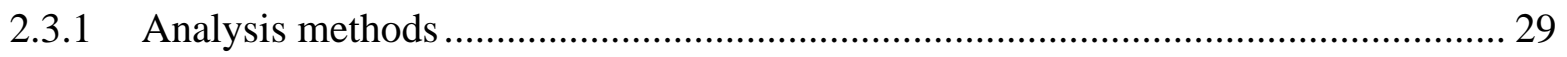




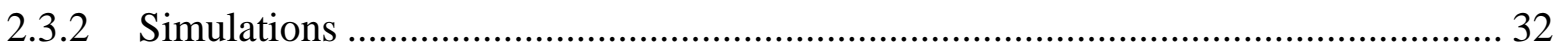

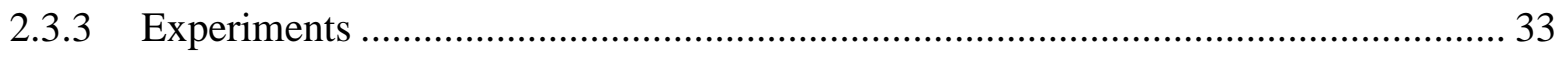

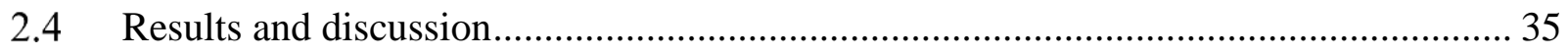

2.4.1 Probing the structural properties of non-resolvable, spherical absorbers ................. 35

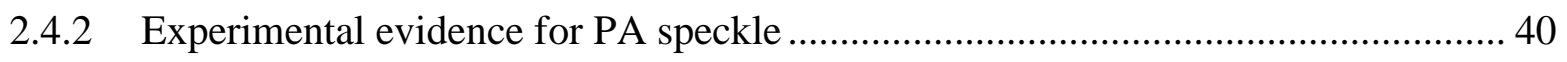

2.4.3 Photoacoustic speckle from tumor vasculature....................................................... 43

2.4.4 Remarks on the nature of speckle in photoacoustic imaging................................... 46

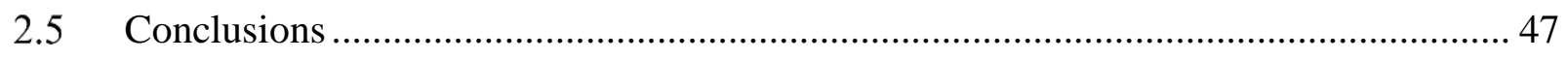

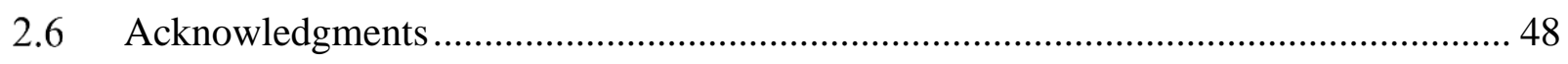

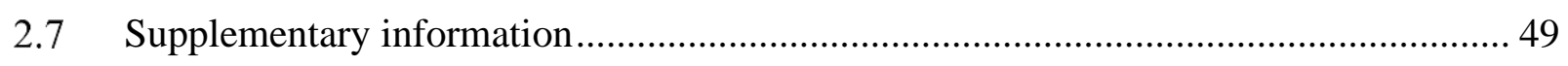

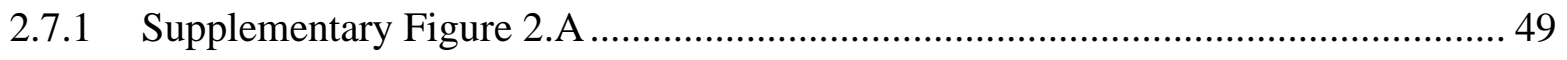

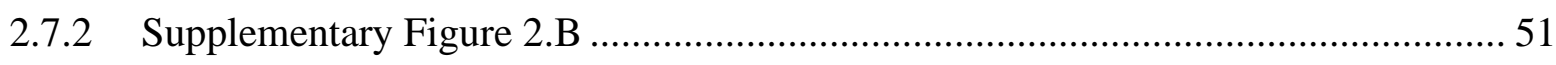

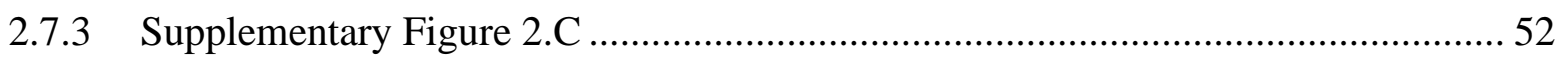

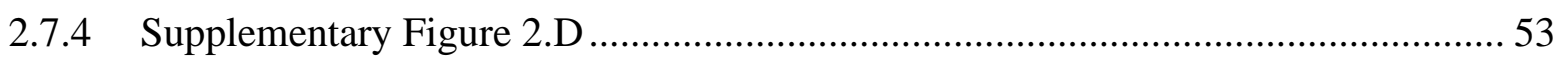

3 Photoacoustic imaging of cancer treatment response: Early detection of therapeutic effect from thermosensitive liposomes......................................................................................... 54

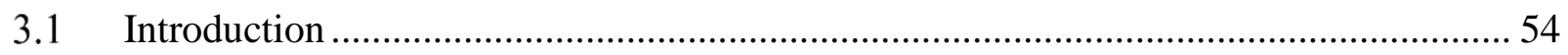

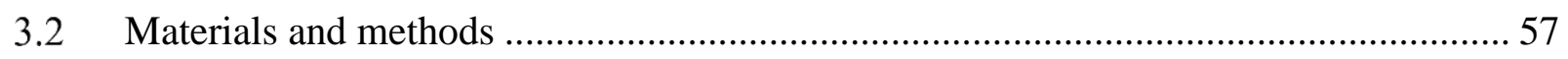

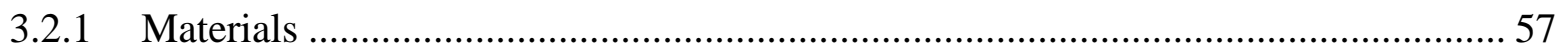

3.2.2 Preparation of HaT-DOX liposomes ................................................................... 57

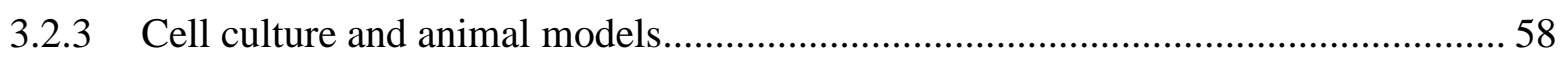

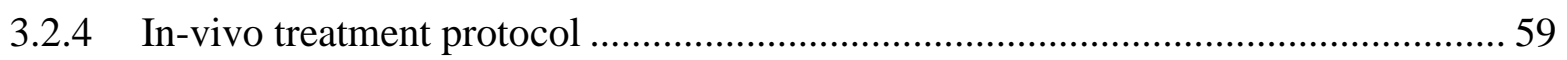

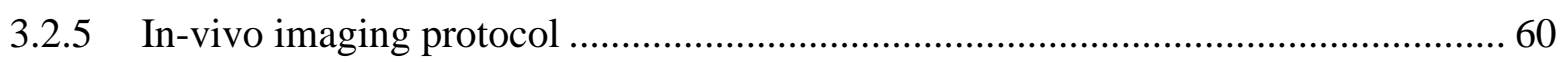

3.2.6 Ultrasound and photoacoustic imaging and data processing ……........................... 60

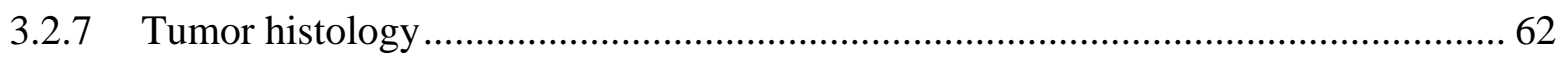

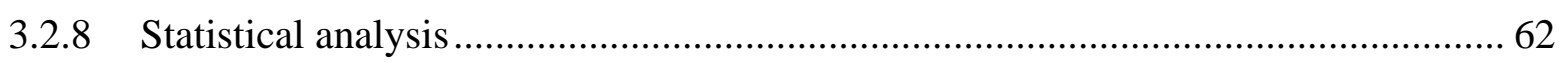

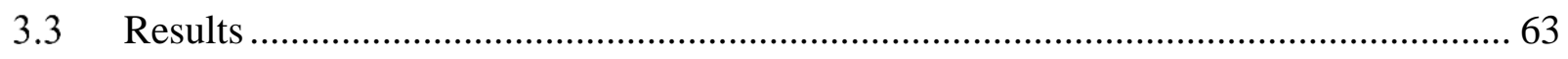

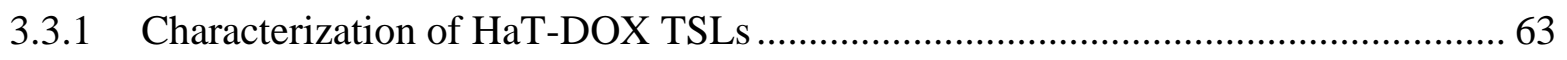

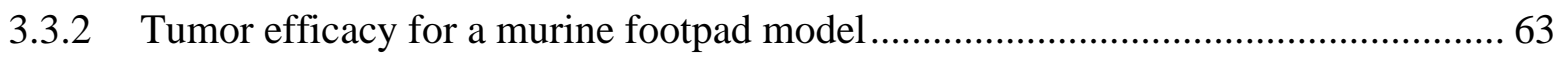

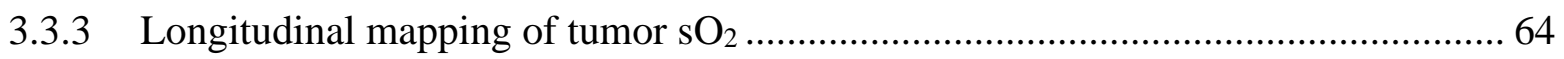

3.3.4 Correlation between early changes in $\mathrm{sO}_{2}$ and treatment efficacy ........................... 69

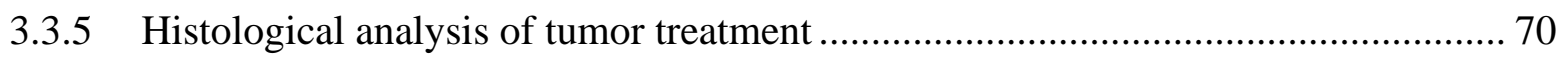

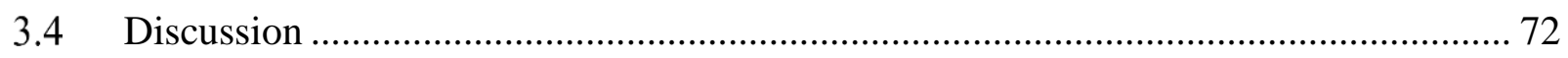

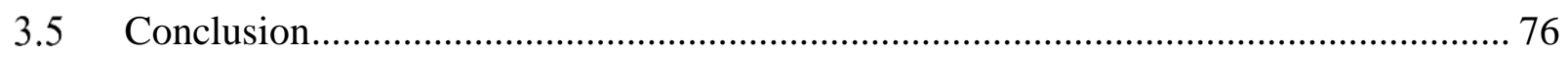

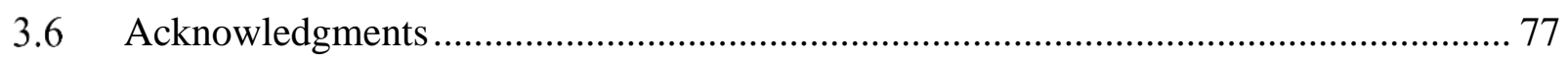




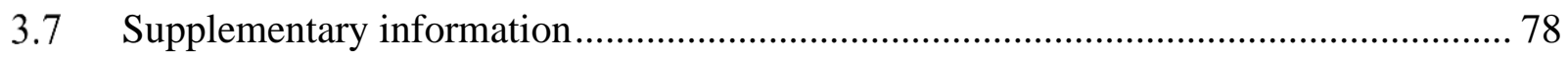

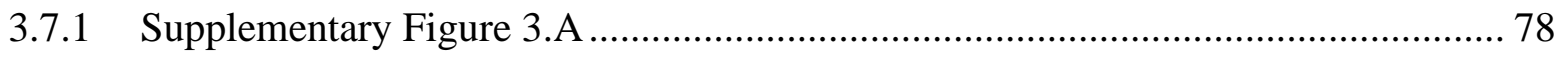

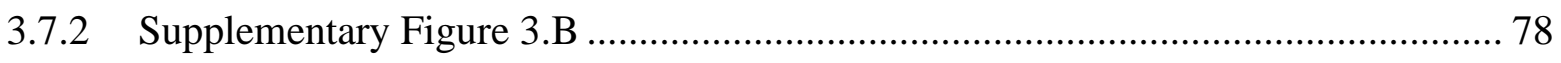

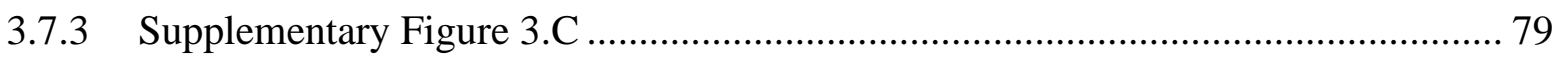

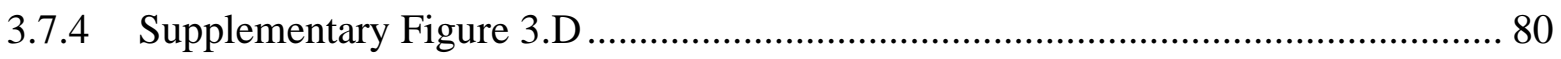

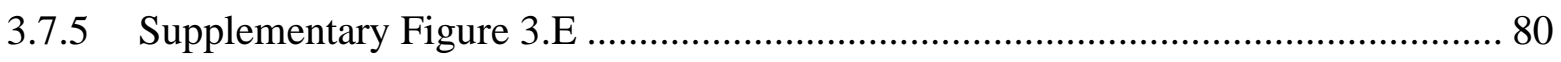

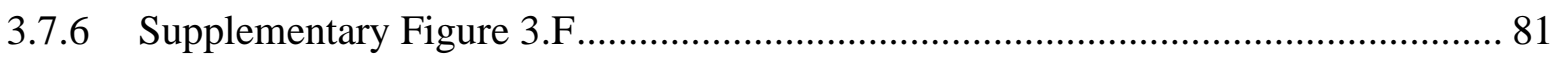

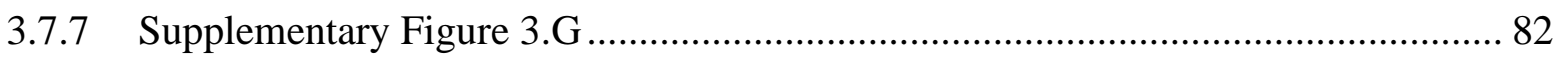

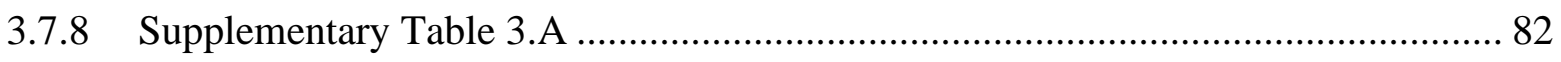

4 Photoacoustic spectral characterization of cancer treatment response: Correlation with changes in tumor oxygenation ............................................................................................................. 83

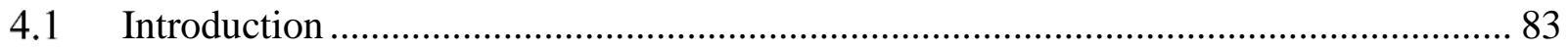

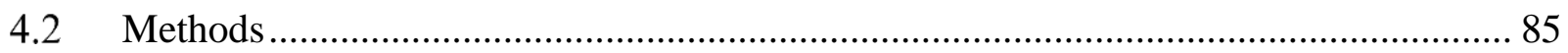

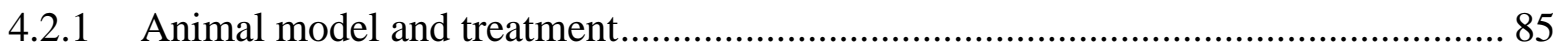

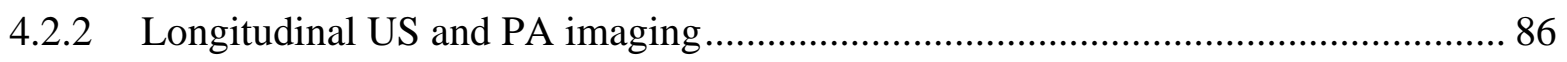

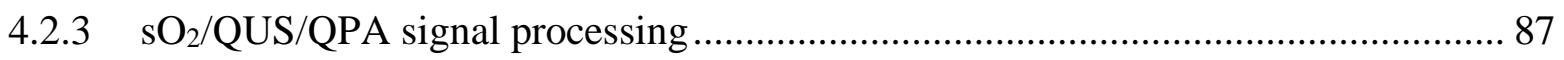

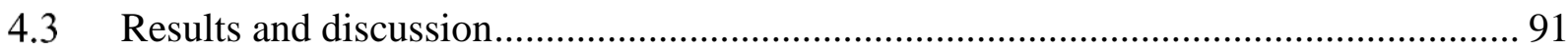

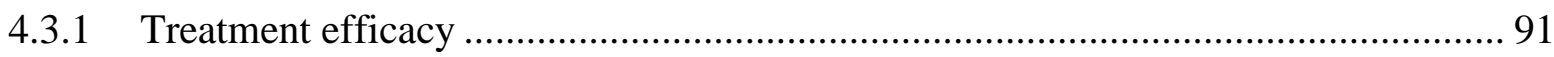

4.3.2 HaT-DOX tumor oxygenation and PA normalized power spectra ........................... 92

4.3.3 Saline tumor oxygenation and PA normalized power spectra ................................. 94

4.3.4 Correlation of QPA parameters with tumor oxygenation ....................................... 95

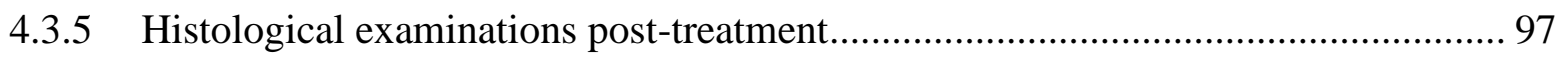

4.3.6 Quantitative ultrasound for detecting tumor cell death.......................................... 99

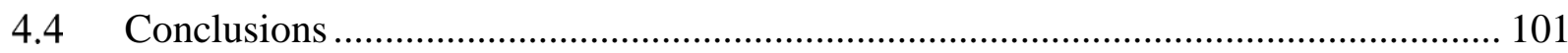

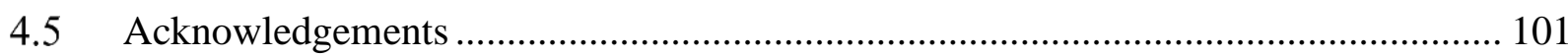

5 Photoacoustic imaging biomarkers for monitoring biophysical changes during nanobubble-mediated radiation treatment ................................................................................. 102

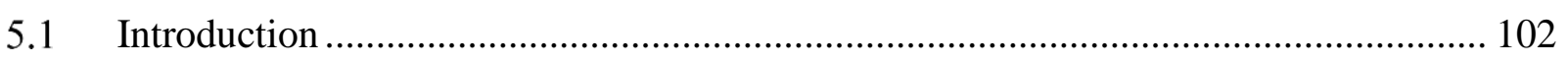

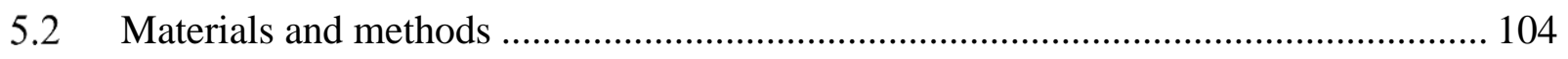

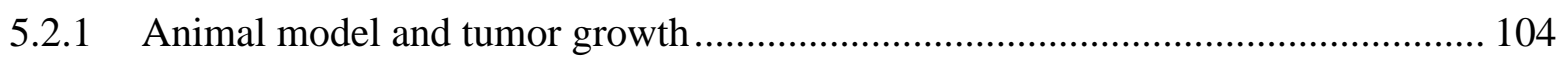

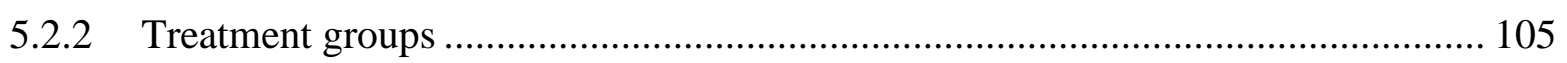

5.2.3 Ultrasound-activated microbubble and nanobubble treatments............................ 105

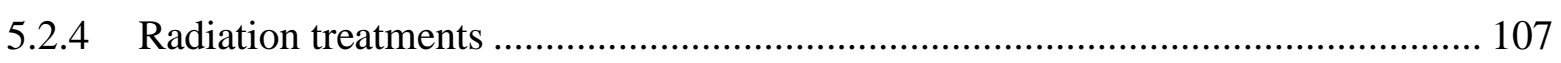

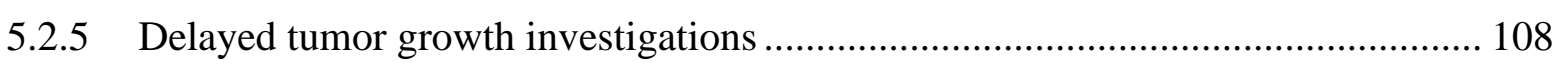

5.2.6 Photoacoustic imaging and biomarkers ............................................................. 108 
5.2.7 Histological analyses and correlations with PA imaging .................................... 111

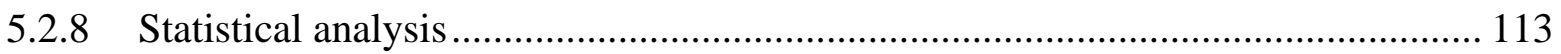

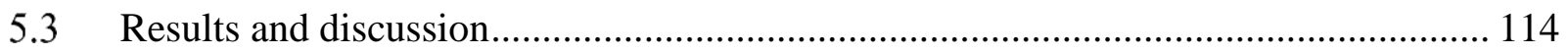

5.3.1 Quantification of micro/nanobubble treatment response ...................................... 114

5.3.2 Changes in functional biomarkers during treatment ........................................... 116

5.3.3 Changes in structural biomarkers during treatments .......................................... 119

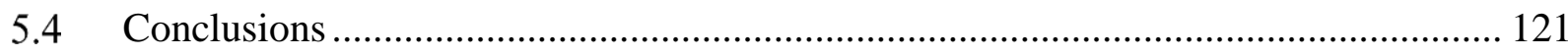

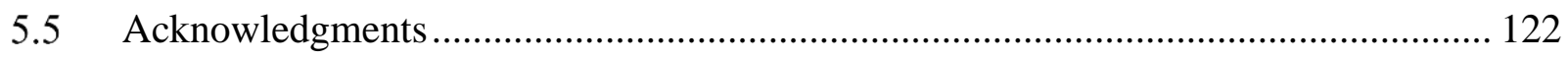

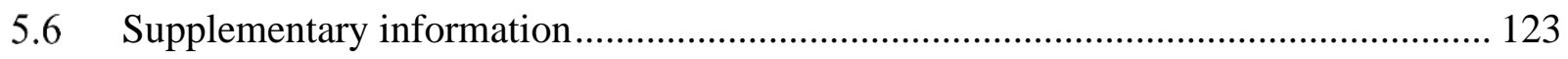

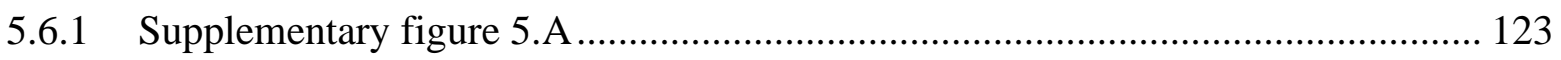

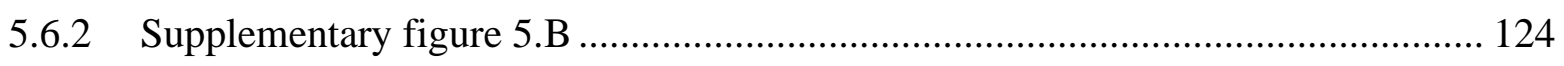

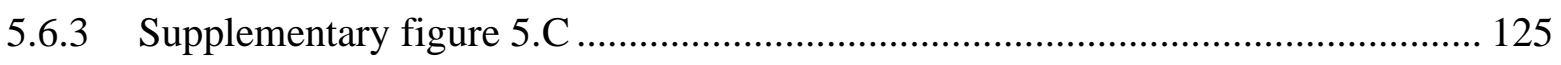

6 Conclusions and future directions.................................................................................. 126

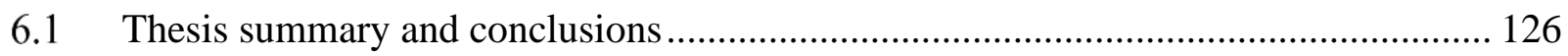

6.1.1 Formation of PA speckle and the derivations of biomarkers................................. 126

6.1.2 Tumor $\mathrm{sO}_{2}$ changes early after HaT-DOX cancer treatment .................................. 127

6.1.3 Oxygenation and frequency biomarkers can differentiate treatment response ....... 128

6.1.4 The biophysical interpretation of PA biomarkers ................................................ 128

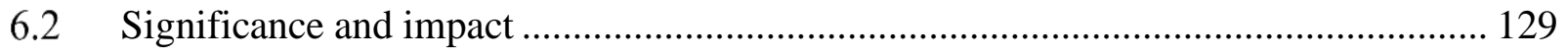

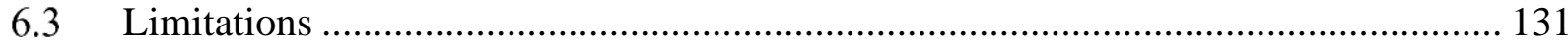

6.4 Future directions............................................................................................ 133

6.4.1 Blood flow and metabolic oxygen consumption measurements............................ 133

6.4.2 Imaging the extracellular matrix of tumors ......................................................... 136

6.4.3 Strengthening the predictive power of PA biomarkers ........................................... 138

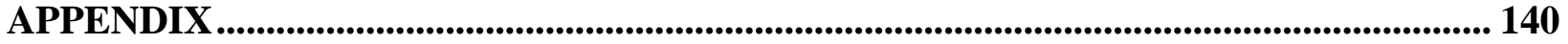

A.1 Permissions to reproduce copyrighted material …………............................................ 140

A.1.1 Chapters 2 and 4 permissions from Elsevier ...................................................... 140

A.1.2 Chapter 3 permission from PLOS....................................................................... 141

REFERENCES............................................................................................................................... 144 


\section{LIST OF TABLES}

Table 1-1: Summary of clinical imaging modalities used for clinical treatment monitoring. Abbreviations: CT - computed tomography; PET - positron emission tomography; SPECT - single positron emission tomography; MRI - magnetic resonance imaging; DCE-US - dynamic enhanced contrast ultrasound.

Table 1-2: Representative imaging biomarkers currently used for clinical decision-making in oncology. Abbreviations: TNM - tumor, lymph node, metastasis; XR - X-ray; DXA - dual-energy X-ray absorptiometry; AUC - area under the curve; FDG fludeoxyglucose; $\mathrm{SUV}_{\max }$ - maximum standardized uptake value of FDG tracer. ${ }^{18} \mathrm{~F}$ -

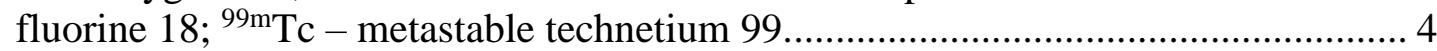

Table 5-1: Experimental treatment groups for the MB and NB experiments......................... 105 


\section{LIST OF FIGURES}

Figure 1-1: Pictorial representation of the tumor microenvironment illustrating the features of tumor blood vessels and implications of their structural organization in cancer therapy. Adapted from reference [56].

Figure 1-2: Overview of PA imaging. Short (nanosecond) laser pulses delivered through an illumination source (denoted by S) illuminate tissues which may contain endogenous or exogenous chromophores. The light absorption from the chromophore biomolecules thermoelastically induces an initial pressure rise which subsequently propagates as ultrasonic waves. An acoustic detector (denoted by D) receives the acoustic waves which are then reconstructed to form a PA image. Adapted from reference [82]. 10

Figure 1-3: Optical absorption coefficients for commonly used endogenous chromophores in PA imaging. Adapted from reference [82]. 11

Figure 1-4: PA imaging configurations. (a) Optical resolution photoacoustic microscopy, (b) Acoustic resolution photoacoustic microscopy and (c) Acoustic resolution photoacoustic tomography. Abbreviations: US - ultrasound; MO - microscope objective, UST - ultrasound transducer, Vis - visible, NIR - near infrared. Adapted from reference [93]. 12

Figure 1-5: Representative clinical PA imaging applications. (a) Overview of the Twente PA mammoscope for breast cancer imaging (left) and a maximum amplitude projection image (right) showing a breast lesion (red). (b) PA imaging of oral vasculature showing blood vessels of the human lip (left), corresponding projection image (middle) and image of a deeper region. (c) 3D PA imaging of palm vessels (left) and the system schematics (right). (d) US/PA imaging of human thyroid (left) and the system schematics (right). Multispectral optoacoustic imaging of (e) inflammatory bowel disease, (f) human carotid artery bifurcation and (g) sentinel lymph node using indocyanine green exogenous contrast agents. Adapted from reference [94]........... 14

Figure 1-6: Typical reconstruction of PA images. (a) Upon the absorption of a single wavelength $\lambda$, the time-domain ultrasound signals recorded from $n$ acoustic detectors are used to reconstruct a PA image which reveals only structural information. (b) This procedure is then repeated for every $\lambda$ used to illuminate the tissue and each pixel amplitude $p o$ at every location in the image $x o, y o$ is (c) spectrally unmixed using dedicated algorithms from known chromophore spectra. (d) The a single-wavelength grayscale frame obtained at $800 \mathrm{~nm}$ reveals the PA image of the forearm of a healthy volunteer while the spectral unmixing quantifies the content of (e) hemoglobin, (f) lipid and (g) water present. The pseudo color image intensity denotes relative chromophore concentration. The scale bars represent $5 \mathrm{~mm}$ while $x$ and $y$ denote the lateral and axial directions relative to the transducer. Adapted from reference [82]. 15

Figure 1-7: (a) Theoretical time domain PA solutions for a fluid layer, cylinder and sphere. The dashed lines show the analytical solutions while the solid lines show the same signals 
after a bandpass filter (200-500 MHz bandwidth) emulating the transducer response is applied. $\tau$ denotes the dimensionless retarded time (courtesy of Dr. Michael Moore, Ryerson University). Experimentally measured time domain PA signals for (b) red blood cells (RBCs) at two different orientations relative to a $400 \mathrm{MHz}$ single element transducer and (c) a melanoma cell. (d) PA power spectra for the signals shown in (b) and (c). Adapted from reference [130]. 17

Figure 1-8: Representative examples of optical (top row) and acoustic (bottom row) resolution PA imaging for cancer treatment monitoring. (a) Single wavelength maximum amplitude projection PA images of tumors before and after treatment with a vascular disrupting agent (adapted from reference [138]). (b) Oxygen saturation $\left(\mathrm{sO}_{2}\right)$ images of tumors before and after a single 9 Gy radiation treatment dose. Scale bars denotes $2 \mathrm{~mm}$ (adapted from reference [137]). 18

Figure 1-9: US and PA B-mode images and RF signals from a gelatin-based phantom containing spherical carbon beads (1-12 $\mu$ m diameter). The phantom is imaged with a $40 \mathrm{MHz}$ center frequency linear array illuminating the sample at $680 \mathrm{~nm}$.

Figure 2-1: Formation of PA speckle. (a) 3D visualization of the geometry of a numerical phantom containing randomly positioned spherical absorbers of different diameter. (b) Typical non-bandlimited PA signals from absorbers of three different diameters. (c) Bandlimited version of PA signals shown in (b). The range of frequencies is determined by the ultrasound detector used. (d) Representative, beamformed PA RF line with contributions from all absorbers. (e) Resultant PA B-mode image obtained

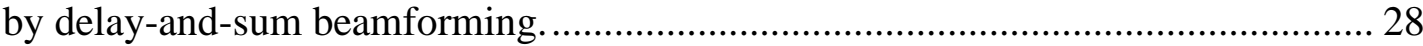

Figure 2-2: Overview of analysis techniques. (a) Representative PA RF signal (red) and its envelope (black). (b) Temporal domain analysis obtained through envelope statistics (top box). (c) Frequency domain analysis obtained through the Fourier transform of PA signals yields radiofrequency spectroscopy (middle box) and cepstral analysis (bottom box). 31

Figure 2-3: Simulation results for the envelope statistics method. (a) PA Bmode images of $5 \mu \mathrm{m}$ spherical bead phantoms for increasing b/rv. (b) Corresponding PA signal envelope histograms fitted to the Rayleigh, Generalized Gamma (GG) and Nakagami (NG) statistical distributions. (c) GG $a$, (d) NG $m$ and (e) NG $\Omega$ parameters plotted as a function of size and concentration. 36

Figure 2-4: Simulation results for the radiofrequency spectroscopy method. PA B-mode images for (a) monodisperse and (b) polydisperse collection of beads at a concentration of 10 beads per resolution volume. SS as a function of (c) bead radius and concentrations and (d) polydispersity (controlled by the $\theta$ parameter). 37

Figure 2-5: Simulation results using the cepstral analysis method. (a) Geometries of phantoms for various grid $(\mathrm{G})$ to random $(\mathrm{R})$ ratio and $(\mathrm{b})$ their corresponding PA B-mode images. (c) Power cepstrum of the 15:85\% phantom with star denoting the location of the first 
peak. (d) Comparison of the estimated absorber with the true spacing for various

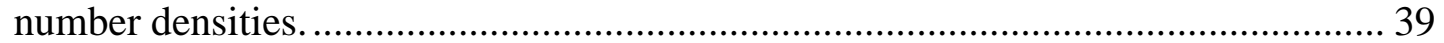

Figure 2-6: Effects of the imaging transducer on PA speckle size. PA B-mode images from speckle phantoms imaged with the (a) Ultrasonix RP, (b) VevoLAZR and the SASAM at (c) 200 and (d) $400 \mathrm{MHz}$. (e) Comparison between estimated speckle size and the spatial resolution of each system. (f) A representative 2D ACVF function from the VevoLAZR. (g) The lateral cross-section of the ACVF was used to estimate speckle size by measuring its FWHM. 41

Figure 2-7: Speckle analysis techniques applied to experimental phantoms. Nagakami fits to the PA signal envelope for the phantoms imaged at (a) $5 \mathrm{MHz}$, (b) $40 \mathrm{MHz}$, (c) $200 \mathrm{MHz}$ and (d) $400 \mathrm{MHz}$. The calculated SNR and Generalized Gamma $m$ parameter are provided on top of each figure. For the VevoLAZR $40 \mathrm{MHz}$ probe, the three analysis techniques presented in this paper, namely (e) Envelope statistics, (f) Radiofrequency spectroscopy and (g) Cepstral analysis were applied to phantoms consisting of 3 and $15 \mu \mathrm{m}$ polystyrene beads. The arrow denotes a peak forming for the $15 \mu \mathrm{m}$ absorber phantom.

Figure 2-8: PA speckle from simulated and experimentally measured tumor vasculature. (a) Geometry of the vascular tree alongside the PA B-mode for the numerical simulations of breast tumor vasculature. The average vessel diameters used for the simulated vasculature were $37.8 \mu \mathrm{m}$ and $48.2 \mu \mathrm{m}$ at 7 and 14 days, respectively, and were all based on histological sectioning of in-vivo tumors. (b) US and PA images $(750 \mathrm{~nm})$ of in-vivo EMT-6 tumors at $7(\mathrm{n}=3)$ and $14(\mathrm{n}=4)$ days post-inoculation. (c)+(e) Spectral slope and (d)+(f) power cepstra for the numerical simulations of tumors modeling vascular growth and the in-vivo EMT-6 tumors. The arrows denote the location of the largest amplitude cepstral peaks. 45

Figure 3-1: Schematic of the mode of action of a temperature sensitive liposome (TSL) for intravascular release. The TSL passes through normal unheated vasculature intact (a), but on reaching the heated tumor (b) drug is released in a burst-release fashion, creating a high local drug concentration which permeates into the tumor tissue...... 55

Figure 3-2: Tumor growth plots for mice treated with 1 hour of hyperthermia (HT) and an intravenous dose of (a) HaT-DOX $(n=10)$ or $(b)$ Saline $(n=12)$. Tumors were grown subcutaneously in the footpad of the right hind limb and changes in size were measured regularly with calipers. HT-HaT-DOX treatments were dosed at $10 \mathrm{mg}$ DOX/kg. The dashed line (-----) represents the endpoint due to tumor load. The $\dagger$ symbol indicates mice which reached a premature endpoint due to tumor ulceration or lack of sufficient mobility. The f symbol indicates all mice that were sacrificed for histology at 7 days post-treatment. 64

Figure 3-3: Representative sO2 maps. These are shown for HT-HaT-DOX (top row) and HTSaline (bottom row) treated tumors at 30 min pre-treatment (1st column), $2 \mathrm{~h}$ posttreatment ( 2 nd column) and 7 days post-treatment ( 3 rd column). The scale bar ( $2 \mathrm{~mm})$ and $\mathrm{sO} 2$ color bar (0-100\%) apply to all sO2 maps shown. 
Figure 3-4:Oxygenation histograms. (a) Average sO2 histograms at 30 min pre-treatment and 30 min, $2 \mathrm{~h}, 5 \mathrm{~h}, 24 \mathrm{~h}$ and 7 day timepoints post-treatment for HT-HaT-DOX $(\mathrm{n}=12)$ and HT-Saline $(n=15)$. Two time courses have been plotted to compare the treatments studied using the mode (b) and mean (c) averages of the histogram data plotted in (a) relative to their starting values at $30 \mathrm{~min}$ pre-treatment. Error bars represent the standard deviation on the pixel count for each $\mathrm{sO} 2$ value from each mouse which had 21 different histograms per imaging timepoint. The black arrows represent the points at which the treatments were made (i.e. defined in this plot as $0 \mathrm{~h}$ ). Datapoints that show a drop in $\mathrm{sO} 2$ which is significantly different to pre-treatment are represented by $*$ where $\mathrm{p}<0.05$.

Figure 3-5: Size and oxygenation relationships. Correlation between the changes in the size of the tumor treated with (a) HT-HaT-DOX and (b) HT-Saline at endpoint (from day 0) and the changes in mean $\mathrm{sO} 2$ between the values observed for 30 min pre-treatment and 2 $\mathrm{h}$ post-treatment. Each point is the average of $21 \mathrm{sO} 2$ histograms at the $2 \mathrm{~h}$ timepoint. The major and minor axes of each ellipse represent the standard deviations of the change in $\mathrm{sO} 2$ and change in tumor size, respectively. $\dagger$ identifies a datapoint for a HT-HaT-DOX treatment that did not show regression, nor a characteristic drop in $\mathrm{sO} 2$ at $2 \mathrm{~h}$ post-treatment. 70

Figure 3-6: Tumor histology. Representative sections of footpad tumor harvested at the $2 \mathrm{~h}$ timepoint and stained with CD31 (red), DAPI (blue) and FITC-lectin (green) for (a) HT-HaT-DOX, and (b) HT-Saline treated mice. The same sections displaying the regions defined as FITC-positive tumor (green), FITC-negative tumor (orange) and normal tissue (maroon), after processing with Definiens software for (c) HT-HaTDOX and (d) HT-Saline. Column scatter plots: Relative FITC-positive areas following quantification with a Definiens analysis at (e) $2 \mathrm{~h}$ and (f) 7 days post-treatment; Significance is represented by $*$ where $\mathrm{p}<0.0005$. 71

Figure 3-7: Proposed mechanism and levels of oxygen saturation (sO2) following treatment with a burst-release TSL such as HaT-DOX triggered with mild-hyperthermia. The timecourse is represented as a number of snapshots which appear sequentially from A-E. 75

Figure 4-1: Schematic for generating tumor oxygenation maps and PA spectral parameters. The ROI segmented from the US images is applied to the co-registered PA images at 750 and $850 \mathrm{~nm}$ which are then used to generate a tumor sO2 map. Oxygenation histograms of every slice within the tumor provide a quantitative distribution of $\mathrm{sO} 2$ values from which the average mode is calculated. The ROI mask is also applied to the reference phantoms at both wavelengths. The frequency information of the PA phantoms is subtracted from the tumor RF spectra and the normalized power spectra are used to retrieve the spectral parameters. For the US normalized spectra, the US image and reference phantom is used to obtain the same parameters. 88

Figure 4-2: Average, relative tumor growth as a function of time post-treatment. Each point represents the average and standard deviation (error bars) of the relative tumor size for 
6 HaT-DOX treated mice and 7 Saline control mice. The HaT-DOX non-responder

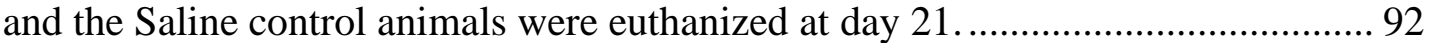

Figure 4-3: HaT-DOX-treated mice (a) tumor oxygenation histograms and the (b) normalized power spectra for PA images obtained at 750 (top) and $850 \mathrm{~nm}$ (bottom) for multiple imaging timepoints. The error bars on the histograms represent the standard deviation on the pixel count for each $\mathrm{sO} 2$ value obtained from 21 different histograms per timepoint per mouse. The power spectra are averages of at least $100 \mathrm{RF}$ lines per 21 tumor slices per timepoint ( 6 total) per mouse ( 5 total). All linear regressions had a Chi-squared value $\geq 0.9$. Treatment is abbreviated as $t x$ 93

Figure 4-4: Saline-treated mice (a) tumor oxygenation histograms and the (b) normalized power spectra for PA images obtained at 750 (top) and $850 \mathrm{~nm}$ (bottom) for multiple imaging timepoints. The error bars on the histograms represent the standard deviation on the pixel count for each sO2 value obtained from 21 different histograms per timepoint per mouse. The power spectra are averages of at least $100 \mathrm{RF}$ lines per 21 tumor slices per timepoint (6 total) per mouse ( 7 total). All linear regressions had a Chi-squared value $\geq 0.9$. Treatment is abbreviated as tx....

Figure 4-5: Oxygen saturation ( $\mathrm{sO} 2)$ mode versus the PA spectral slope (PA SS). The PA SS is computed for $750 \mathrm{~nm}$ (top row) and $850 \mathrm{~nm}$ (bottom row) and for HaT-DOX (a) responders $(n=5)$ and $(b)$ non-responder $(n=1)$ and (c) Saline $(n=7)$. Each dot represents the average SS across at least 100 PSnorm within 21 tumor slices per timepoint, per mouse. 96

Figure 4-6: Tumor histology obtained $2 \mathrm{~h}$ post-treatment. (a) Representative images of HaT-DOXtreated tumor blood vessels ( $2 \mathrm{~h}$ post-treatment) stained with CD-31 (red and yellow) showing the leakage of FITC-lectin (green) in their vicinity (red ellipse). The width of the image is $10 \mathrm{~mm}$ and the scale-bar in the zoomed inset is $100 \mathrm{~mm}$. (b) Representative HaT-DOX and Saline tumors showing the proportion of FITC-lectin leakage (dark gray) outside of the tumor area (orange). Normal tissue is the teal color surrounding the tumor and the slide background is light gray. For more details on how these images were obtained refer to reference [43]. (c) Distribution of the size of tumor blood vessels for HaT-DOX and Saline measured at the $2 \mathrm{~h}$ timepoint. 99

Figure 4-7: The correlation between US SS and sO2 mode for HaT-DOX (a) responders and (b) non-responder and (c) Saline. Each dot represents the average SS across at least 100 $\mathrm{RF}$ spectra within 21 tumor slices per timepoint per mouse. 100

Figure 5-1: Ultrasonic treatment and PA imaging setups. (a) Ultrasonic bubble activation setup. The tumor is positioned at the focus of the therapeutic ultrasonic transducer. (b) PA imaging platform illustrating the prone imaging position of the mouse during imaging. The mice are kept at physiological temperature through a heated stage and a heating lamp. 107

Figure 5-2: PA imaging biomarkers of treatment response. The effects of the combination of ultrasound-simulated $\mathrm{MB}$ or NB treatments with radiation can be probed using PA 
imaging biomarkers (functional and structural) and can be correlated with histological metrics of tumor cells and blood vessels. Treatment and tumor illustrations were created with Biorender.com. Abbreviations: $\mathrm{MB}=$ microbubble; $\mathrm{NB}=$ nanobubble; $\mathrm{sO} 2=$ oxygen saturation; $\mathrm{HbT}=$ total hemoglobin . 110

Figure 5-3: Histological metrics of tumor vessels and cells. (a) CD31 staining of tumor blood vessels can be used to estimate both the (b) vascularity of tumors by measuring the CD31 positive area and (c) the vascular density and vessels size by computing the total number and size of each vessel, respectively. The red pseudo color denotes the vessels and green and yellow denote the background and tissue-free areas, respectively. The cell-free areas are assessed by the presence of the hematoxylin nuclear counterstain. (d) TUNEL staining can be quantified by measuring the (e) TUNEL positive area in the tumor in order to estimate the extent of cell death. Red and yellow pseudo colors represent different intensities of TUNEL staining. Abbreviations: CD31 = cluster of differentiation $31 ;$ AUC $=$ area under curve; TUNEL $=$ Terminal deoxynucleotidyltransferase-mediated-dUNP-biotin nick end labeling. ................. 113

Figure 5-4: PA imaging and histological biomarkers of treatment response. Change in (a) PA estimations in tumor $\mathrm{sO}$, (b) CD31 positive area and (c) TUNEL positive area relative to the untreated control as a function of treatment and imaging timepoint ( $\mathrm{sO} 2$ only). The error bars denote the standard error of the mean change for all animals belonging in that treatment group. Statistical significance is doted by * $(\mathrm{p}<0.05)$ and n.s. denotes not significant. The $\mathrm{sO} 2$ comparisons denote statistical significance between the $2 \mathrm{~h}$ and $24 \mathrm{~h}$ imaging timepoints. For every biomarker, representative sO2 maps, CD31 and TUNEL histological images are shown for the untreated control and the $\mathrm{NB}+\mathrm{US}+8 \mathrm{~Gy}$ treatment. Axial and lateral distances in the sO2 maps are in relation to the imaging transducer. 115

Figure 5-5: Impact of treatments on oxygenation metrics. Correlations between the changes in (a) $\mathrm{HbT}$ and $\mathrm{sO} 2$, (b) CD31 and sO2, (c) CD31 and $\mathrm{HbT}$, (d) TUNEL and sO2, and (e) TUNEL and sO2. Each parameter change is measured at 24 hours post-treatment and is calculated relative to the untreated control, for all treatment groups. Error bars denote the standard error of the mean change for all animals belonging in that treatment group. 117

Figure 5-6: Tumor growth delay data for single nanobubble and radiation treatments. The tumor volumes were normalized to the size of the tumor on day 1 post-treatment, for each respective group. The error bars denote the standard error of the mean. 118

Figure 5-7: Impact of treatments on tumor vascularity. Correlations between the changes in (a) Vessel perimeter and Vascular density, (b) Vascular density and sO2, (c) Vascular density and HbT, (d) Vascular density and SS at $750 \mathrm{~nm}$ and (e) Vascular density and $\mathrm{SS}$ at $850 \mathrm{~nm}$. Each parameter change is measured at 24 hours post-treatment and is calculated relative to the untreated control, for all treatment groups. Error bars denote the standard error of the mean change for all animals belonging in that treatment group. 
Figure 6-1: Schematic highlighting the work in this thesis. The development of PA imaging treatment biomarkers that induce vascular damages enable monitoring of cancer treatment response. Histological assessments of vasculature and tumor cell death enable the identification of treatment responders. Images were adapted from references [150], [151] and [293]. Illustrations were created with Biorender.com. 130

Figure 6-2: (a) Photoacoustic microscopy image of total hemoglobin concentration $\left(\mathrm{C}_{\mathrm{Hb}}\right)$ from the skin of a mouse. Scale bar denotes $500 \mu \mathrm{m}$. (b) $\mathrm{sO}_{2}$ map of the area indicated by the dashed box in the hemoglobin image. (c) PA flow map of dashed box area shown in (b). The pseudo colors indicate the direction of blood flow relative to the scanning direction. Scale bar denotes $125 \mu \mathrm{m}$. (d) Profile of blood flow speed across the dashed line in (c) showing the parabolic flow profiles expected from arteries and veins. Adapted from reference [326]. 135

Figure 6-3: The contributions of collagen to the biological activities of cancer cells. Adapted from

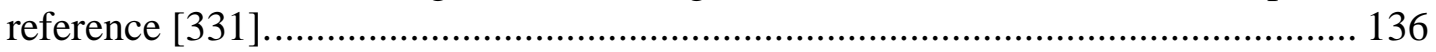

Figure 6-4: (a) PA imaging setup for blood collagen phantom gels. (b) Representative co-registered US and PA images (at $850 \mathrm{~nm}$ ) of a blood collagen phantom. (c) Validation of the unique spectral unmixing algorithm to quantify collagen. Error bars represent the standard deviation of the mean, with 60 measurements per phantom. The $\mathrm{r}^{2}$ denotes the goodness of the linear fit. (d) Representative PA and histology collagen maps of mouse kidneys with progressively higher degrees of fibrosis at day 7 and day 14 compare to sham.

Figure 6-5: (a) Representative US B-mode of a locally advanced breast cancer tumor and (b) its corresponding ROI for the tumor core (red) and a $5 \mathrm{~mm}$ margin thickness (blue). (c) The corresponding spectral intercept (SI) parametric image which can differentiate (d) good vs. (e) poor treatment response. Scale bar denotes $1 \mathrm{~cm}$. Adapted from reference [336]. 139 


\section{ABBREVIATIONS}

\begin{tabular}{|c|c|}
\hline $\mathrm{ACVF}$ & Autocovariance function \\
\hline $\mathrm{BF}$ & Beamformed \\
\hline $\mathrm{BL}$ & Bandlimited \\
\hline B-mode & Brightness mode \\
\hline CD31 & Cluster of differentiation 31 \\
\hline $\mathrm{CT}$ & Computed tomography \\
\hline $\mathrm{dB}$ & Decibel \\
\hline DOSI & Diffuse optical spectroscopy imaging \\
\hline EMT-6 & Epithelial mesenchymal transition breast cancer cells \\
\hline F-mode & Frequency mode \\
\hline FWHM & Full width at half maximum \\
\hline GG & Generalized gamma \\
\hline $\mathrm{H} \& \mathrm{E}$ & Hematoxylin and eosin \\
\hline HaT-DOX & Heat activated cytotoxic doxorubicin \\
\hline $\mathrm{Hb}$ & Deoxyhemoglobin \\
\hline $\mathrm{HbO}$ & Oxyhemoglobin \\
\hline HBS & Hepes buffered saline \\
\hline HT & Hyperthermia \\
\hline LTSL & Lyso-lipid temperature sensitive liposome \\
\hline MB & Microbubble \\
\hline MBF & Midband fit \\
\hline MRI & Magnetic resonance imaging \\
\hline NB & Nanobubble \\
\hline NG & Nakagami \\
\hline OA & Optoacoustics \\
\hline $\mathrm{OPO}$ & Optical parametric oscillator \\
\hline PA & Photoacoustics \\
\hline PAM & Photoacoustic microscopy \\
\hline PBS & Phosphate buffered saline \\
\hline PC3 & Prostate cancer cells \\
\hline PET & Positron emission tomography \\
\hline QPA & Quantitative photoacoustics \\
\hline QUS & Quantitative ultrasound \\
\hline $\mathrm{RF}$ & Radiofrequency \\
\hline ROI & Region of interest \\
\hline SASAM & Saarland scanning acoustic microscope \\
\hline $\mathrm{SD}$ & Standard deviation \\
\hline SNR & Signal to noise ratio \\
\hline $\mathrm{sO}_{2}$ & Oxygen saturation \\
\hline SS & Spectral slope \\
\hline TSL & Temperature sensitive liposome \\
\hline TUNEL & Deoxynucleotidyltransferase-mediated-dUNP-biotin nick end labeling \\
\hline US & Ultrasound \\
\hline uTSL & Ultrafast temperature sensitive liposome \\
\hline
\end{tabular}




\section{LIST OF SYMBOLS}

$\begin{array}{ll}\text { Symbol } & \text { Definition } \\ \phi & \text { Velocity potential }[\mathrm{m} / \mathrm{s}] \\ \beta & \text { Isobaric thermal expansion coefficient }[1 / \mathrm{C}] \\ \rho & \text { Density }\left[\mathrm{kg} / \mathrm{m}^{3}\right] \\ C_{P} & \text { Specific heat capacity }[\mathrm{J} / \mathrm{K}] \\ r & \text { Spatial position }[\mathrm{m}] \\ H & \text { Heating function }\left[\mathrm{J} / \mathrm{m}^{3}\right] \\ t & \text { Time }[\mathrm{s}] \\ p & \text { Pressure }[\mathrm{Pa}] \\ \lambda & \text { Optical wavelength of illumination } \\ \mu_{a}^{\lambda} & \text { Optical absorption coefficient }\left[\mathrm{cm}^{-1}\right] \\ g_{T} & \text { Transducer time domain impulse response } \\ \varpi_{0} & \text { Transducer center frequency }[\mathrm{MHz}] \\ \xi & \text { Transducer }-6 \text { dB bandwidth }[\mathrm{MHz}] \\ D(\alpha) & \text { Transducer directivity function } \\ \alpha & \text { Angle relative to the center of each transducer element }[\circ] \\ J_{1} & \text { First order Bessel function } \\ k & \text { Wavenumber }[1 / \mathrm{m}] \\ R & \text { Transducer aperture }[\mathrm{m}] \\ p_{B L}^{B F}(t) & \text { Directivity-corrected, beamformed, bandlimited pressure } \\ \tau_{n} & \text { Time delay at the } \mathrm{n}^{\text {th }} \text { transducer element } \\ I & \text { Reconstructed photoacoustic image } \\ \mathcal{H} & \text { Hilbert transform } \\ P S(f) & \text { Power spectrum } \\ P S_{f i t}(f) & \text { Normalized power spectrum } \\ f & \text { Frequency }[\mathrm{MHz}] \\ U\left(\varpi_{x}, \varpi_{z}\right) & \text { Two-dimensional discrete Fourier transform of a photoacoustic image } \\ \sigma & \text { Rayleigh distribution fit parameter } \\ m & \text { Nakagami parameter } \\ \Omega & \text { Nakagami scaling parameter } \\ c / v & \text { Generalized gamma distribution fit parameters } \\ \theta & \text { Standard deviation of absorber size distribution } \\ \mathcal{F} & \text { Fourier transform } \\ C(t) & \text { Power cepstrum } \\ \varepsilon_{H b} & \text { Extinction coefficient of deoxyhemoglobin }\left[\mathrm{M}^{-1} \cdot \mathrm{cm}^{-1}\right] \\ \varepsilon_{H b o} & \text { Extinction coefficient of oxyhemoglobin }\left[\mathrm{M}^{-1} \cdot \mathrm{cm}^{-1}\right] \\ p_{S A} & \text { Photoacoustic signal amplitude } \\ U(f, z) & \text { Ultrasound power spectrum as a function of frequency } f \text { at location } z \\ U S_{n o r m} & \text { Normalized ultrasound power spectrum } \\ P(f, z, \lambda) & \text { Photoacoustic power spectrum as a function of optical wavelength } \lambda \\ P S_{n o r m P A} & \text { Normalized photoacoustic power spectrum } \\ & \end{array}$




\section{CO-AUTHORSHIP STATEMENT}

This dissertation contains materials published $(n=3)$ or submitted for publication $(n=1)$ in the following four peer-reviewed journals:

1. E Hysi, MN Fadhel", MJ Moore*, J Zalev, EM Strohm and MC Kolios. "Insights into photoacoustic speckle and applications in tumor characterization" Photoacoustics 2019; 14 3748 - doi.org/10.1016/j.pacs.2019.02.002

*These authors contributed equally to this work.

$\mathrm{EH}$ and MCK were responsible for the conception of the study. EH developed the theoretical formulation of speckle and planned, supervised and executed all experiments including all formal analysis. MNF assisted with numerical simulation developments and MJM performed the photoacoustic microscopy experiments. JS assisted with the development of the vascular tree models and EMS assisted with the planning and analysis of microscopy measurements. EH wrote the original draft of the manuscript with review and editing contributed by all authors. This work was performed under the supervision of MCK.

2. JP May*, E Hysi*, LA Wirtzfeld, E Undzys, S-D Li and MC Kolios. "Photoacoustic imaging of cancer treatment response: Early detection of therapeutic effect from thermosensitive liposomes" PLOS ONE 2019; 11(10): e0165345 - $\underline{\text { doi.org/10.1371/journal.pone.0165345 }}$

*These authors contributed equally to this work.

EH, JPM, SDL and MCK conceptualized the study and designed the experiments. EH, JPM and LAW executed all experiments with EU helping with animal injections. JPM and SDL designed the thermosensitive liposome formulation. EH developed and implemented the in vivo oxygen saturation algorithm and performed all formal analysis. EH and JPM wrote the original draft of the manuscript with review and editing provided by all authors. This work was performed out under the supervision of SDL (drug formulation) and MCK (photoacoustic imaging). 
3. E Hysi, LA Wirtzfeld, JP May, E Undzys, S-D Li and MC Kolios. "Photoacoustic spectral characterization of cancer treatment response: Correlation with changes in tumor oxygenation" Photoacoustics 2017; 5: 25-35 - $\underline{\text { doi.org/10.1016/j.pacs.2017.03.003 }}$

EH conceptualized the analysis method and developed frequency-based approach. EH, LAW, JPM and EU executed all experiments. JPM and SDL designed the thermosensitive liposome formulation. EH performed all formal analysis with assistance from LAW. EH wrote the original draft of the manuscript with editing and review contributions provided by all authors. This study was performed under the supervision of MCK.

4. E Hysi, MN Fadhel", Y Wang *, JA Sebastian, A Giles, GJ Czarnota, AE Exner and MC Kolios. "Photoacoustic imaging biomarkers for monitoring biophysical changes during nanobubblemediated radiation treatment" Photoacoustics 2020, under review.

*These authors contributed equally to this work.

$\mathrm{EH}$ and MCK were responsible for the conception of the study. MNF assisted with in vivo imaging and YW was responsible for nanobubble formulations. JA assisted with analysis of the histological data. AG was responsible for tumor growth and execution of bubble and radiation treatments. GJC provided access to the animal imaging and treatment facilities as well as scientific guidance. AAE designed the original formulation of nanobubbles and assisted with the experimental design. EH developed all (imaging and histological) biomarker algorithms, performed all formal analysis and wrote the original draft of the manuscript. MCK supervised all aspects of the study. 


\section{CHAPTER 1}

\section{Introduction}

\subsection{Cancer and personalized medicine}

Cancer is a complex disease that is being treated with multiple approaches. Despite the fact that treatments such as surgery, chemotherapy and radiation therapy have become standards of care [1], overall survival rates remain modest. The five year survival rates for pancreatic (4\%), lung (15\%), liver (7\%) and glioblastoma (5\%) still remain "abysmally low" [2]. Even breast and prostate cancers which are highly responsive to treatments (5-year survival rate $>80 \%)$, respond very poorly at later stages. In Canada, women with locally advanced breast cancer exhibit only a 5-10\% complete response rate, dying within 2 to 5 years [3]. Prostate cancer treatment prolongs life but often causes impotence, incontinence, hot flashes and even risk of bone fractures [4]. Moreover, cancer treatments are accompanied by a wide array of side effects. The systemic cytotoxicity of chemotherapy regimens often results in acute and delayed nausea, ulcerations of the mouth and mild cognitive impairments. Furthermore, the rapid increase in the number of older adults ( 25 to 35 million in 1980-2000, 72 million by 2030) suggests that there will be a significant increase in cancer diagnoses [5].

These statistics and shortcomings have prompted a shift in the paradigm of cancer care. Treatment approaches are no longer simply driven from tumor histological grading. Human genome mapping advances suggest that "access to genetic information will radically and dramatically improve the way medicine is practiced..." [6]. Understanding cancer causation and progression has resulted in the discovery and development of molecularly targeted drugs for personalized, precision treatments. These rely on individualized drug administration based on prognostic and predictive markers of response [7]. The importance of the aforementioned approaches to cancer treatment has prompted the announcement of the Precision Medicine Initiative ${ }^{\circledR}$, a 2016 enterprise which will invest $\$ 215$ million in disease (including cancer) management by taking into account the generic variability, environment and lifestyle of each patient [8]. The ultimate hope is that such a personalized strategy in cancer therapy will ultimately replace the "one-size-fits-all" conventional approach and increase the overall survival rates. 


\section{CHAPTER 1}

\subsection{Personalized treatments require personalized imaging}

New drugs or experimental treatments challenge clinicians to understand efficacy on a patient-bypatient basis. In order for a new treatment to gain enough traction, its preliminary effectiveness must be assessed with inexpensive and readily available means. Personalized cancer treatments have already revealed problems caused by the tumor molecular heterogeneity which in turn leads to individualized and dynamic treatment-induced changes in tumor morphology and structure [9]. Current treatment assessment approaches might not be suitable for handling the complexities of personalized cancer treatments. Personalized imaging for treatment monitoring should be sensitive to biophysical changes in tissue related to the proposed drug's activity. There is a clear need for imaging modalities which are sensitive to specific, early biomarkers (structural and functional) of treatment response and that can be used to provide fast, reliable feedback on treatment efficacy.

\subsection{Current imaging standards of treatment monitoring}

The goal of treatment monitoring is the classification of interventional outcome. Conventionally, solid tumor response is assessed by standard anatomical-based imaging [10]. The Response Evaluation Criteria In Solid Tumors (RECIST) has standardized how response should be assessed when determining the effect of cytotoxic agent(s) [11]. The endpoint of any cancer treatment must be measured in a uniform, objective and reproducible manner using a standardized method of assessment [12]. RESIST is a resource which can be implemented using readily available standardized imaging modalities. These criteria can be used to identify ineffective therapies, which should be abandoned early in favor of treatments which benefit patients and reduce the burden on the health-care system [13]. As such, medical imaging has played an integral role is utilizing RECIST to determine the treatment response [14] and key modalities are reviewed in Table 1-1. The use of these modalities in oncology has given rise to imaging biomarkers which are described in detail in Section 1.4. 


\section{CHAPTER 1}

Table 1-1: Summary of clinical imaging modalities used for clinical treatment monitoring. Abbreviations: CT - computed tomography; PET - positron emission tomography; SPECT single positron emission tomography; MRI - magnetic resonance imaging; DCE-US - dynamic enhanced contrast ultrasound.

\begin{tabular}{|c|c|}
\hline Modality & Features/Examples of treatment monitoring/Challenges \\
\hline X-Ray CT & $\begin{array}{l}\text { - } \\
\text { - } \\
\text { - } \\
\text { - }\end{array}$ \\
\hline PET/SPECT & $\begin{array}{l}\text { - Capture of positrons (PET) or gammas (SPECT) from intravenous isotopes } \\
\text { - Low spatial resolution (6-8 mm) requires co-registration with CT [13], [15] } \\
\text { - FDG uptake can differentiate response within } 24 \text { hours but limited to slowly } \\
\text { growing tumors (prostate, thyroid and endocrine) [16] } \\
\text { - Expensive on-site facilities, SPECT isotopes hard to couple to probes [10] }\end{array}$ \\
\hline MRI & $\begin{array}{l}\text { - } \text { Proton relaxation measures rate of water exchange within cells } \\
\text { - Apoptosis-induced membrane size and integrity measured at } 36 \text { hours [17] } \\
\text { - Contrast agents can assess angiogenesis and vessel permeability [18] but high } \\
\text { concentrations are needed since resolution depends on signal to noise ratio } \\
\text { [13] }\end{array}$ \\
\hline DCE-US & $\begin{array}{l}\text { - Can assess blood volume, vessel size and flow speed changes through } \\
\text { microbubble contrast agents } \\
\text { - Metastatic renal carcinoma responders identified } 2 \text { weeks post-treatment [19] } \\
\text { - Imaging only limited to intravascular space due to size of microbubbles }\end{array}$ \\
\hline
\end{tabular}

\subsection{Biomarkers of cancer treatment response}

Broadly defined, a biomarker is any objectively and quantitatively measured characteristic that can serve as an indicator of "normal biological processes, pathologic processes, or pharmacologic responses to a therapeutic intervention" [20]. The current FDA-NIH Biomarker working group recognizes examples of biomarkers to be molecular, histological, imaging or physiological characteristics [21]. The measurement of biomarkers during cancer therapies has the potential to relate clinical responses to the effect that intervention has on molecular and cellular pathways. Moreover, biomarkers can aid in the evaluation of novel cancer therapies [22], thus assisting with a more rigorous selection of drug compounds for clinical trials. Table 1-2 summarizes a selected list of imaging biomarkers which have been use in various aspects of clinical oncology. These biomarkers have found widespread use in oncology whether its screening for the disease, diagnosis and staging, targeting and guiding surgical/radiotherapy treatments and predicting and monitoring therapeutic efficacy and toxicity [23]. With the exception of US imaging, the remainder of the modalities summarized in Table 1-2 are relatively expensive, thus limiting the availability and 


\section{CHAPTER 1}

practicability of these modalities for treatment response monitoring. Moreover, conventional approaches using MRI or CT rely on gross changes in tumor size, limiting their feasibility as volumetric changes in the size of tumors sometimes require months of therapy even in the presence of a positive pathological response [24]. Functional imaging biomarkers such as PET require long scan times and the administration of exogenous contrast agents to detect response-related changes in tumors thus constraining how often a patient can be scanned during the course of months-long treatments [25], [26].

Table 1-2: Representative imaging biomarkers currently used for clinical decision-making in oncology. Abbreviations: TNM - tumor, lymph node, metastasis; XR - X-ray; DXA - dual-energy X-ray absorptiometry; AUC - area under the curve; FDG - fludeoxyglucose; $\mathrm{SUV}_{\max }$ - maximum standardized uptake value of FDG tracer. ${ }^{18} \mathrm{~F}$ - fluorine $18 ;{ }^{99 \mathrm{~m}} \mathrm{Tc}$ - metastable technetium 99.

\begin{tabular}{|c|c|c|}
\hline $\begin{array}{l}\text { Imaging } \\
\text { biomarker }\end{array}$ & Imaging modality & Decision-making role \\
\hline Clinical TMN stage & $\begin{array}{l}\text { XR, CT, MRI, } \\
\text { PET, SPECT, US, } \\
\text { endoscopy }\end{array}$ & Prognostic in nearly all cancers \\
\hline Bone scan index & SPECT & Prognostic in nearly all cancers \\
\hline Objective response & CT, MRI, PET & $\begin{array}{l}\text { Guides decision to continue, discontinue, or switch } \\
\text { therapy }\end{array}$ \\
\hline $\begin{array}{l}\text { ACR BI-RADS } \\
\text { breast morphology }\end{array}$ & Mammography & Diagnosis of breast cancer \\
\hline $\begin{array}{l}\text { Left ventricular } \\
\text { ejection fraction }\end{array}$ & Scintigraphy, US & Safety biomarker which can also guide therapy \\
\hline T-Score & DXA & $\begin{array}{l}\text { Safety biomarker which can guide prescription of } \\
\text { medication for therapy-induced bone and hair loss }\end{array}$ \\
\hline $\begin{array}{l}\text { Uptake of }{ }^{99 \mathrm{~m}} \mathrm{Tc}- \\
\text { sestamibi }\end{array}$ & SPECT & $\begin{array}{l}\text { Rapid clearance can predict lack of tumor response } \\
\text { to chemotherapy }\end{array}$ \\
\hline Splenic volume & CT, MRI & $\begin{array}{l}\text { Assessment of response in patients with } \\
\text { myelofibrosis }\end{array}$ \\
\hline Microbubble AUC & US & $\begin{array}{l}\text { Pharmacodynamic and putative predictive imaging } \\
\text { biomarker }\end{array}$ \\
\hline$\Delta^{18}$ F-FDG SUV $\max$ & PET & $\begin{array}{l}\text { Monitoring imaging biomarkers for other of } \\
\text { therapies and used in dose-finding and to provide } \\
\text { evidence of treatment efficacy }\end{array}$ \\
\hline
\end{tabular}

\subsubsection{Emerging imaging biomarkers}

In this section, two imaging modalities that have shown potential for non-invasive, contrast-free cancer treatment monitoring are described. Both modalities can generate biomarkers that overcome some of the limitations described in Section 1.4. 


\section{CHAPTER 1}

\subsubsection{Quantitative ultrasound}

Quantitative ultrasound (QUS) is a variant of conventional ultrasound imaging. It relies on the analysis of the frequency components of the radiofrequency (RF) signals whose envelope amplitude is typically used to generate B-mode images. Since its inception in the 1970's, QUS has been used to provide more quantitative, system independent readings [27]-[29]. US images are affected by a large number of parameters such as gain compensation, low-pass filters, transducer aperture and dynamic range compression. One cannot rely on the B-mode images for quantitative comparisons between different machines or even the same machine when different settings are used. The latter becomes important in treatment monitoring where multiple users/settings might be utilized for longitudinal imaging sessions [30]. QUS removes the system dependencies by relying on reference phantoms whose images are used to normalize each acquisition. As such, post-normalization QUS can be used to generate parameters that provide information about the size, concentration, shape and spatial distribution of ultrasonic scatterers from a wide array of tissue types [31].

In cancer treatment monitoring, QUS has been used to detect chemotherapy-induced cellular death (apoptosis) [32]-[34]. This led to recent clinical studies where QUS parameters yield meaningful information about the structural properties of cancer tissue and cells as they undergo treatment [24], [35]. The technique has been successful in detecting treatment response of locally advance breast cancer patients with $100 \%$ sensitivity and $93 \%$ specificity one week after the administration of treatment. However, QUS can only measure the tissue structural changes that occur during treatment. There is evidence in the literature that these structural changes are dependent on the tumor and treatment type. This may not allow QUS to be extended towards all treatment types [36]. Moreover, as functional and metabolic changes may appear at earlier time points, QUS may not be able to detect these changes, thus limiting the sensitivity and robustness of the approach for detecting treatment response [37].

\subsubsection{Diffuse optical spectroscopy imaging}

Diffuse optical spectroscopy imaging (DOSI) represents a family of optical imaging techniques that use diffused, near-infrared light to probe the absorption and scattering properties of tissue for the purposes of diagnosis and cancer treatment monitoring [38]. DOSI techniques quantify the 


\section{CHAPTER 1}

photon migration within tissue to calculate bulk tissue optical properties [39], [40]. Unlike QUS, DOSI is sensitive to the metabolic activity of tissue and the detected signals can be related to changes in the water, lipid, and hemoglobin concentrations.

DOSI does not require exogenous contrast agents and it has found broad applications in providing functional information of brain activity [41]. It has also been used to monitor the response of breast cancer patients during neoadjuvant chemotherapy [42]-[44]. These landmark studies suggest that treatment-induced functional and metabolic changes appear earlier than structural changes, thus allowing DOSI to differentiate treatment responders as early as 24 hours post-treatment. An oxyhemoglobin flare present in treatment responders is attributed to the rapid increase in cellular metabolism due to chemotherapeutic-induced cellular damage [43]. At 4 weeks post-treatment, the non-responders show little-to-no changes in the water, oxy/deoxyhemoglobin and scattering power optical properties [42]. The combination of QUS spectral parameters and DOSI-estimated markers of response showed $100 \%$ sensitivity/specificity at 1 week post-treatment [44]. The correlations of DOSI parameters with vascular changes (ex. vessel collapse or angiogenesis) reinforce the idea that it is possible to follow treatment progression by relying on non-anatomical changes in tumors.

However, as an optical imaging modality, DOSI suffers from insufficient spatial resolution (comparable to PET) due to scattering of photons in the ballistic regime, on the order of centimeters. DOSI can probe centimeters deep into tissue, but the chaotic path of diffuse photons in tissue due to scattering renders the image reconstruction mathematically ill-posed, leading to poor spatial resolution [45]. It remains quite difficult for pure optical imaging to attain spatial resolution at depths beyond the optical diffusion limit that surpasses conventional imaging modalities [46]. This presents challenges in identifying the anatomical source of the metabolic changes without assistance from external modalities such as US, mammography, or MRI. Additionally, the commercially available DOSI system is limited to breast studies as it is restricted by the geometry of multiple source/detector pairings [44].

\subsection{Vascular biomarkers of treatment response}

Both QUS and DOSI demonstrate that it is possible to develop imaging biomarkers for cancer treatment response that do not rely on conventional RESIST-based changes in tumor volume. It is 


\section{CHAPTER 1}

well known that many solid tumors are relatively resistant to ionizing radiation, chemotherapy and other non-surgical treatment modalities [47]. This lack of responsiveness is attributed to genetic factors that influence how certain cancer cells respond to drugs or physiological properties created primarily from inadequate and non-uniform vascular networks [48]. In this section, the role of the tumor vasculature in cancer and its implications for therapies are discussed. The need for imaging biomarkers that can monitor the vascular response to cancer treatments is also identified.

\subsubsection{Blood vessels in cancer}

The importance of the vasculature in tumor growth and metastasis has been long recognized [49]. Angiogenesis, or the formation of new blood vessels from pre-existing ones, is a hallmark of cancers. In 1971 Folkman hypothesized that the growth of tumors is angiogenesis dependent, permitting the new blood vessels to bring in fresh nutrients and growth factors thus enabling the tumor mass to expand beyond 1-2 $\mathrm{mm}$ in diameter [50]. This seminal observation has significant implications in understanding cancer development and has been dogma of nearly 50 years of active research in cancer biology and oncology [51]. At the microvascular level, there are significant differences in the organization of tumor vasculature compared normal tissue with implications for therapeutic outcome, as shown in Figure 1-1. Normal tissue vessels branch in a hierarchical fashion to ensure adequate delivery of nutrients to all cells. On the other hand, chaotic tumor angiogenesis creates vascular trees with uneven diameters, random patterns of interconnectivity and shunts thus making some regions of the tumor impermeable to drugs [52]. Furthermore, oversized vascular pores cause fluid leakage into the interstitum. This also creates irregular blood flow patterns which produce conditions of hypoxia and high acidity [53], [54]. At a molecular level, the angiogenesis of tumors may be triggered by hypoxia, hypoglycemia and acidosis, all of which activate proangiogenic proteins that promote the formation of new capillaries by recruiting and simulating endothelial cells [55]. 


\section{CHAPTER 1}

Malformed vasculature inside a tumor turns a bad situation worse (boxes). Flaws in the organization and functioning of blood vessels create barriers that prevent therapies from reaching tumor cells and foster an environment where those treatments are less effective. These unnatural internal conditions also contribute to malignant properties of the cancer itself.

VESSEL ORGANIZATION

II Oversize diameter and chaotic layout create irregular blood flow - Absent or immature vessels make some tumor regions impenetrable
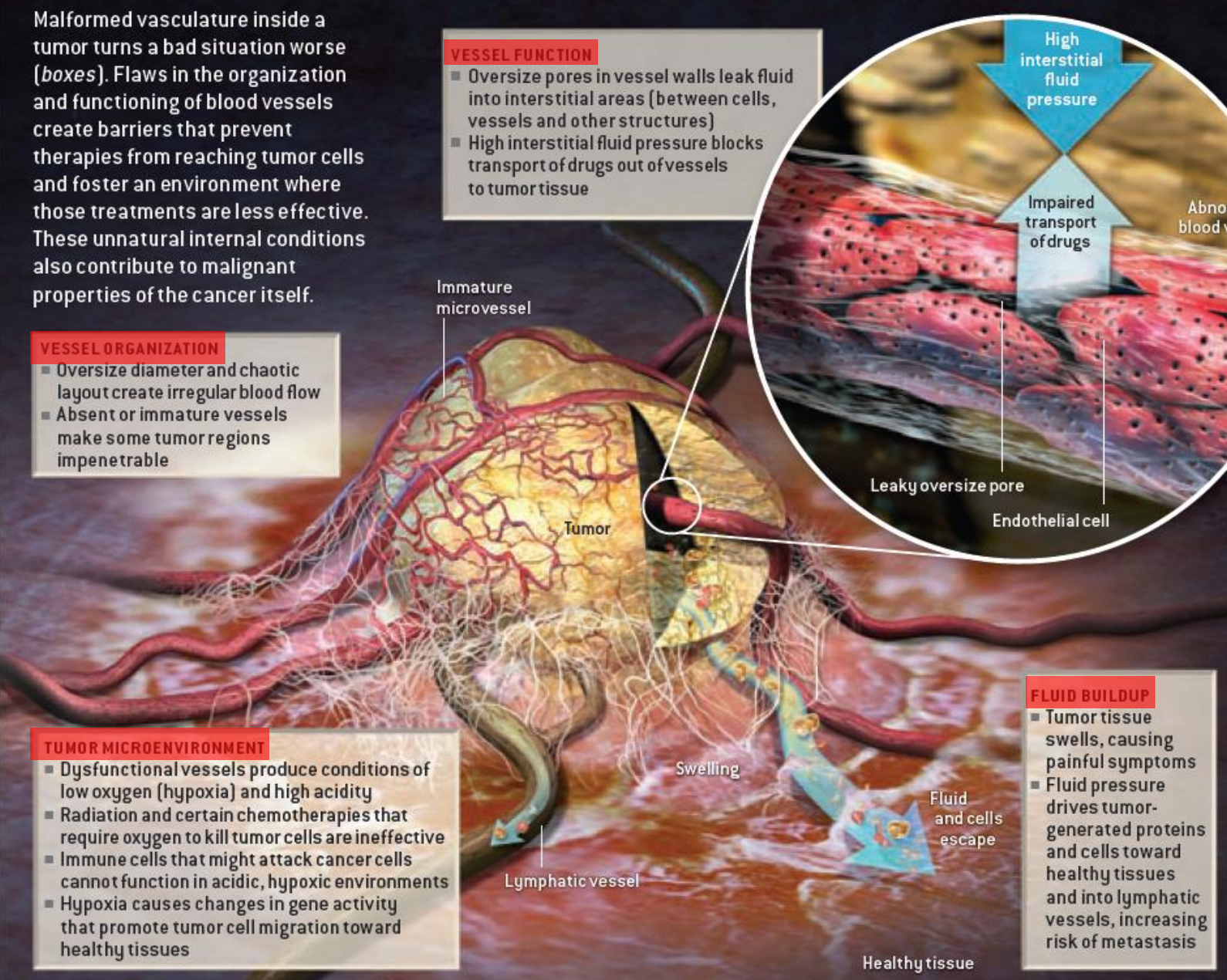

Figure 1-1: Pictorial representation of the tumor microenvironment illustrating the features of tumor blood vessels and implications of their structural organization in cancer therapy. Adapted from reference [56].

\subsubsection{Role of tumor vasculature in cancer therapy}

The tumor vascular architecture has significant implications in cancer therapy (Figure 1-1). The hypoxic areas present significant challenges for radiation therapy since radiation requires the presence of oxygen to function effectively [57]. The highly acidic environment prevents the immune response of natural defense mechanisms and contributes to overall resistance [50]. In addition, hypoxia affects gene activity and promotes metastasis and angiogenic factors [58]. In conjunction with complex hemodynamic flow patterns within the tumor vasculature, it is well recognized that hypoxia and the lack of perfusion leads to poor overall survival rates [48], [59][61]. In fact, the imaging of tumor hypoxia has been proposed as a method for improving our 


\section{CHAPTER 1}

ability to predict outcome in patients that respond poorly to radiotherapy [62]. The interstitial fluid pressure buildup blocks the transport of drugs outside of vessels, preventing therapeutic molecules from reaching the tumor tissue. The fluid buildup also causes swelling pain and physically drives proteins and cells from the tumor towards healthy tissues through lymphatic pathways, promoting metastasis [63]. Several therapeutic approaches have been developed to either shut down the tumor nutrient supply (anti-angiogenic therapy) [64] or re-establish regular flow patterns (normalizing therapy) [65]. The first approach attempts to prevent angiogenesis in tumor growth, while the latter aims to improve the delivery of drugs uniformly throughout the tumor therefore maximizing therapeutic efficiency [66]. Antiangiogenic drugs targeting the vascular endothelial growth factor have been approved by the FDA either as standalone therapies or in combination with chemotherapy for six tumor types [67]. However, their full potential has yet to be realized as the clinical trials have revealed that many cancer patients are either intrinsically resistant to antiangiogenic drugs and do not respond at all. Other patients who respond initially, acquire therapeutic (evasive) resistance after several months of clinical benefit [68], [69]. Moreover, conventional therapies such as radiation and chemotherapy impact the vasculature of tumors, inducing changes to endothelial cells [70]-[73] as well as the blood oxygenation via alterations in hemoglobin levels due to tumor cell metabolism and blood vessel density [74]-[76].

The above-mentioned properties of tumor vasculature suggest that that monitoring vascular changes during treatments could be of considerable benefit. Therapy-induced vascular changes have the potential of serving as surrogate markers of therapeutic efficacy. Biomarkers that revolve around blood vessels can be useful in monitoring angiogenesis, quantifying drug activity/inactivity, facilitating the development of combination therapies and identifying treatment resistance [77]. An imaging modality that is: (1) easy-to-use, (2) inexpensive/accessible and (3) can measure changes that the tumor vasculature undergoes shortly after the administration of treatment does not exist. Given the importance of blood vessels on tumor growth and response to therapy, a modality that can detect in-vivo changes in tumor vasculature could provide clinicians

with immediate feedback, potentially maximizing the impact of the treatment and improving patient outcomes. 


\section{CHAPTER 1}

\subsection{Photoacoustic imaging and its potential}

\subsubsection{Principles of photoacoustic imaging}

The origins of photoacoustic (PA) imaging (which is also referred to as optoacoustic imaging) can be traced back to the 1880 discovery of Alexander Graham Bell who demonstrated the conversion of optical energy to audible sound [78]. No significant progress was made until the invention of the laser which enabled flexibility in the illumination of the sample. First the approach was used in gas spectroscopy and then later in biomedical applications [79], when PA became a modality that combines ultrasonic resolution with optical contrast. Figure 1-2 shows a schematic overview of the PA effect in biological tissue. PA imaging utilizes optical absorption of pulsed laser light to generate mechanical sound waves from the thermoelastic expansion of chromophores [80]. The subsequent sound wave travels within the medium carrying an infinite number of frequencies which are filtered by the transducer or the intervening tissue. Once the sound wave is produced, it follows the same propagation principles in both US and PA imaging. Commercially available US transducers can be used in PA imaging systems [81].

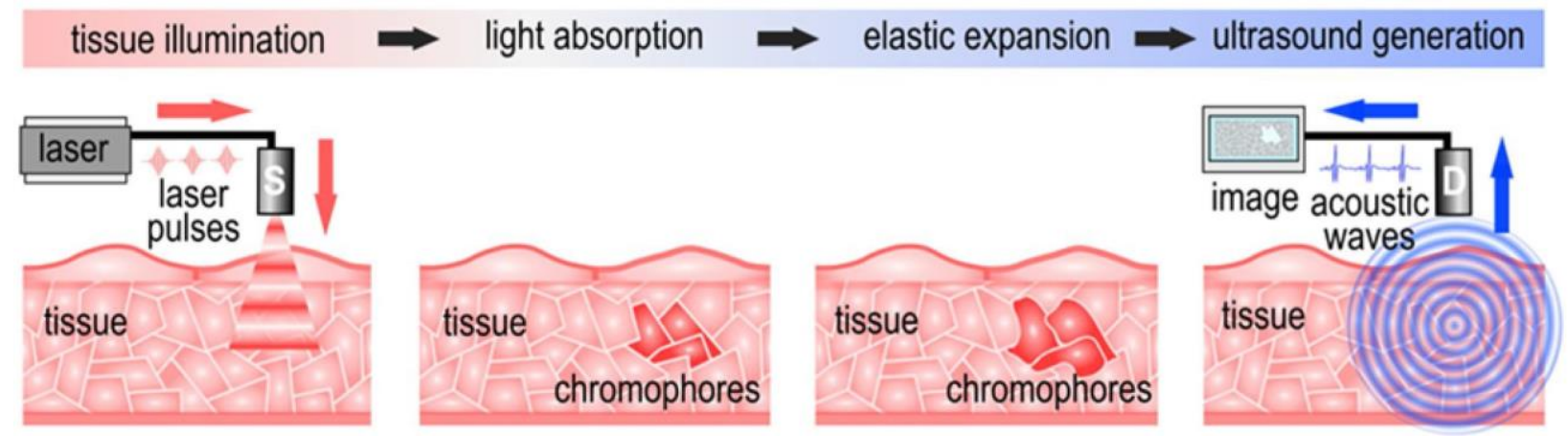

Figure 1-2: Overview of PA imaging. Short (nanosecond) laser pulses delivered through an illumination source (denoted by S) illuminate tissues which may contain endogenous or exogenous chromophores. The light absorption from the chromophore biomolecules thermoelastically induces an initial pressure rise which subsequently propagates as ultrasonic waves. An acoustic detector (denoted by $\mathrm{D}$ ) receives the acoustic waves which are then reconstructed to form a PA image. Adapted from reference [82].

In US imaging, the incident sound wave scatters due to differences in the structural composition of the target and the background (i.e. density and speed of sound). In PA imaging, the strength of the signal heavily depends on the tissue chromophore identity and concentration. In addition to the structural/anatomical information provided by single wavelength illumination, PA imaging also takes advantage of the differential optical absorption of tissue chromophores (Figure 1-3) to probe 


\section{CHAPTER 1}

for the presence of multiple chromophores simultaneously by sweeping the wavelength of illumination [83]. Of great interest is hemoglobin $(\mathrm{Hb})$, the second-most abundant highly absorbing protein (after melanin) localized inside red blood cells (RBCs; 280 million Hb proteins per RBC). With its two oxygenated and deoxygenated states, $\mathrm{Hb}$ exhibits an oxygen-dependent absorption profile [84], introducing a source of contrast in PA images. Due to the abundance of blood in tissue, contrast in PA imaging is typically higher than US images. This has allowed PA imaging to provide functional information by sweeping the laser source to measure the concentration and spatial distribution of various chromophores [85]. Additionally, the US resolution and reduced sound scattering (approximately 1000 smaller than optical scattering) provides PA imaging with better contrast and penetration depth compared to pure optical imaging. The latter relies on the detection of diffuse photons whose chaotic paths in tissue limit spatial resolution and its penetration depth in typical biomedical applications [83].

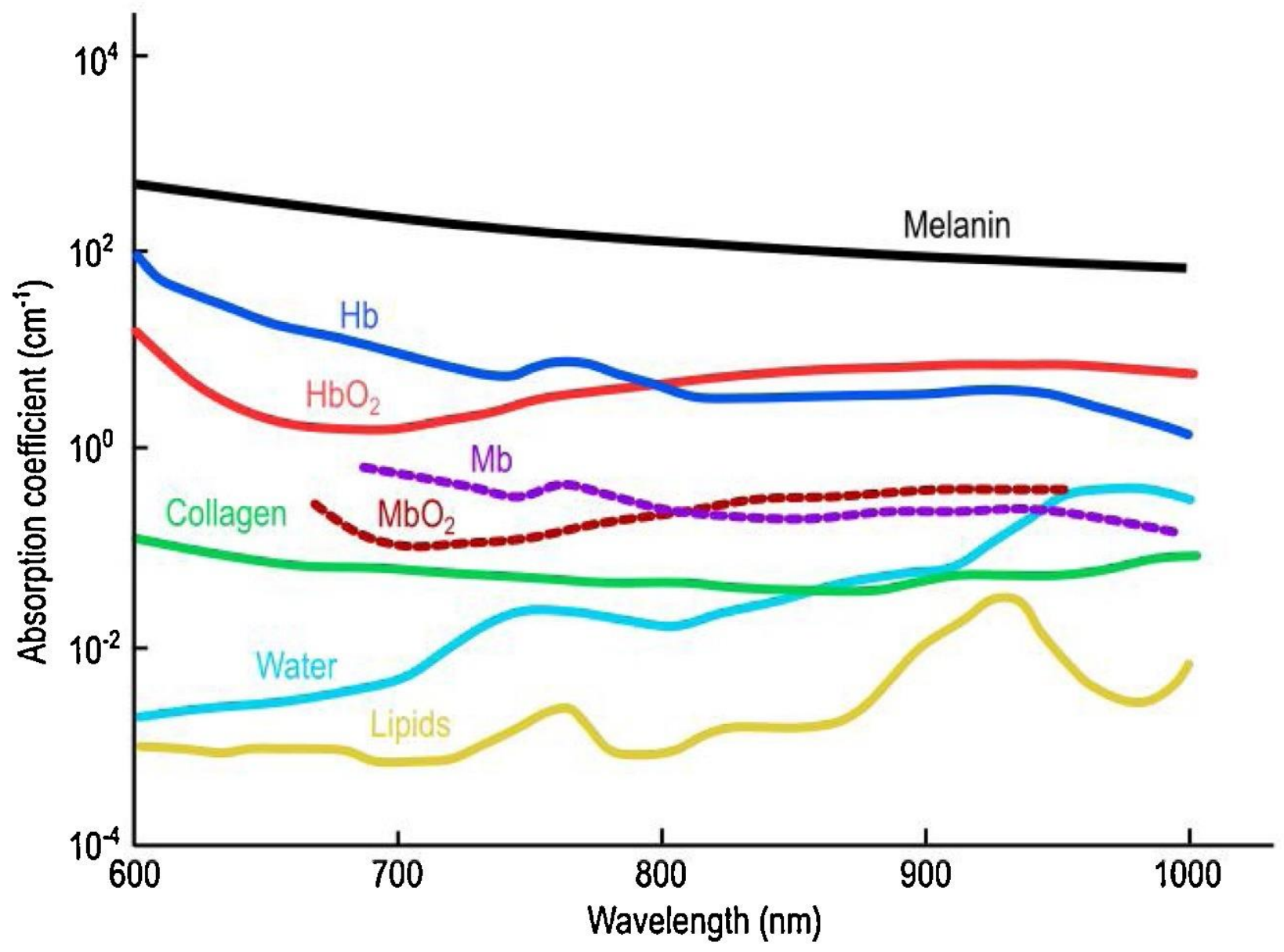

Figure 1-3: Optical absorption coefficients for commonly used endogenous chromophores in PA imaging. Adapted from reference [82]. 


\section{CHAPTER 1}

\subsubsection{Configurations and applications of PA imaging}

Since the late 1980's the field has exponentially grown in terms of publication output as well as breadth of biomedical applications [86]. Advances in light source technologies have contributed to PA imaging systems that are more mobile, tunable, compact and affordable [87]. The scalability of PA imaging illumination and acoustic detection schemes has given rise to multiple configurations which are broadly depicted in Figure 1-4. The focus of the laser source, choice of acoustic transducer and physical orientation relative to one another dictates the biological length scale which can be probed [88]. In optical resolution PA microscopy (Figure 1-4a), the optical excitation focus is co-aligned with the acoustic transducer focus through a layer of silicone oil that acts as an optical/acoustical splitter [89]. This approach can achieve sub-micron resolution but it is restricted to a few hundred microns in depth in order to maintain the optical focusing (depth-toresolution ratio of about 200) [90]. In acoustic resolution PA microscopy (Figure 1-4b), the light is delivered around the transducer to achieve uniform illumination, which also allows for greater penetration. The acoustic focal region determines the spatial resolution (axial and lateral) of the system [91]. To achieve deeper imaging depth required for clinical applications, neither the light nor the sound is focused in acoustic resolution PA tomography (Figure 1-4c), achieving submillimeter resolution at depths of several centimeters through beamforming reconstructions [92].

(a)

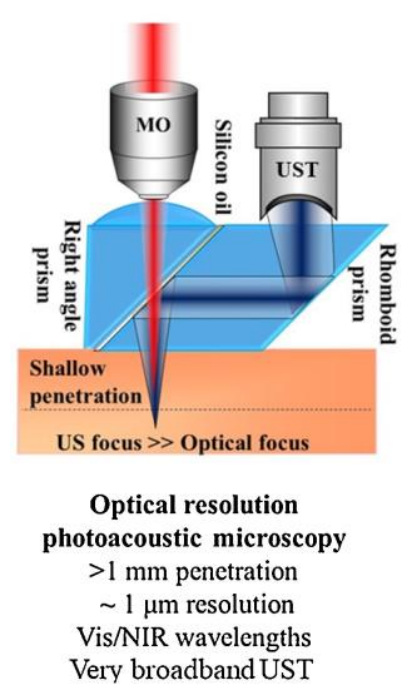

(b)

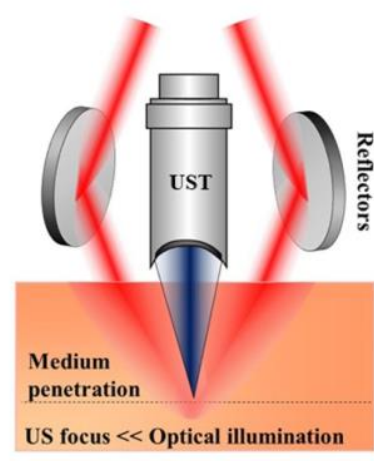

$$
\begin{aligned}
& \text { Acoustic resolution } \\
& \text { photoacoustic microscopy } \\
& \text { Several mm penetration } \\
& \sim 50 \mu \mathrm{m} \text { resolution } \\
& \text { Vis/NIR wavelengths } \\
& \text { BroadbandUST }
\end{aligned}
$$

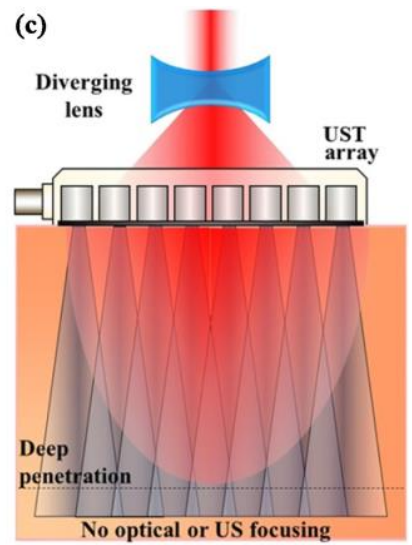

\footnotetext{
Acoustic resolution photoacoustic tomography Several $\mathrm{cm}$ penetration $>250 \mu \mathrm{m}$ resolution NIR wavelengths Standard UST
}

Figure 1-4: PA imaging configurations. (a) Optical resolution photoacoustic microscopy, (b) Acoustic resolution photoacoustic microscopy and (c) Acoustic resolution photoacoustic tomography. Abbreviations: US - ultrasound; MO - microscope objective, UST - ultrasound transducer, Vis - visible, NIR - near infrared. Adapted from reference [93]. 


\section{CHAPTER 1}

Broadly speaking, the microscopy variants of PA imaging shown in Figure 1-4a and Figure 1-4b have already made significant contributions to fundamental life sciences research at multiple biological length scales from the single vessel, single cell, or even subcellular levels [94], [95]. Among numerous applications, worth highlighting is photoacoustic microscopy (PAM) for the non-invasive imaging of the variations in hemoglobin oxygen saturation $\left(\mathrm{sO}_{2}\right)$ in the subcutaneous microvasculature of rats in vivo [96]. Additionally, Wang and colleagues used high-speed, high frame rate PAM to measure the release of oxygen from single red blood cells in the mouse brain [97]. Recent work has demonstrated the capabilities of PAM to explore the intrinsic sensitivity of DNA and RNA for label-free, histology-like assessment of tumor margins in human lumpectomy specimens [98]. Single pulse panoramic photoacoustic computed tomography and spiral volumetric optoacoustic tomography have been developed to achieve real-time, whole-body small animal imaging capabilities for tracking circulating tumor cells [99] and the mapping of exogenous contrast agent distribution [100], respectively. Functional PA imaging contributions include the mapping of the resting-state neuronal functional connectivity in the mouse brain [101]. Genetically encoded, photo-switchable molecular probes with near infrared absorption have been optimized or engineered as PA contrast agents [102]. Optical pH indicators encapsulated inside nanoparticles have been developed to quantify the tumor acidity using multispectral PA imaging [103].

In addition to pre-clinical/fundamental research applications, PA imaging has made significant strides towards clinical translation, achieving imaging depths far beyond those of optical PA microscopy. Figure 1-5 summarizes a few representative PA imaging applications in humans. The early detection of breast cancer through imaging of hypoxia and angiogenesis have yielded very promising clinical data that have the potential to replace mammography (Figure 1-5a) [104]-[109]. Portable imaging systems have also been developed for the superficial detection of oral cancers (Figure 1-5b, [110]) and skin cancer/eczema/dermatitis (Figure 1-5c, [111]). The clinical potential of acoustic resolution PA imaging has also been demonstrated by imaging thyroid tumor vasculature to a depth of $2 \mathrm{~cm}$ (Figure 1-5d, [112]). Multispectral optoacoustic tomography has shown clinical utility in the assessment of Crohn's disease by evaluating the hemoglobin content present during intestinal wall inflammation (Figure 1-5e, [113]). More recently, a hand-held PA

imaging probe allowed for a $2 \mathrm{~cm}^{3}$ field of view imaging of the human carotid artery, opening doors to early assessment of stroke risk through plaque imaging (Figure 1-5f, [114]). Lastly, the 


\section{CHAPTER 1}

use of clinically approved optical contrast agents such as indocyanine green for the PA imaging aids in the identification of sentinel lymph node metastases from melanoma patients [115].

(a)
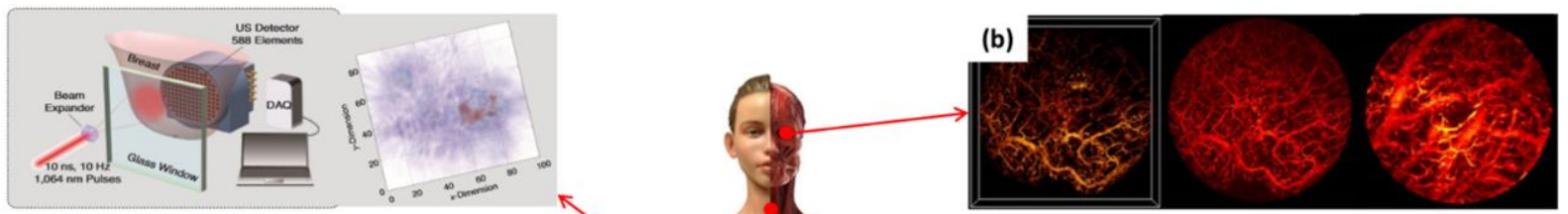

(c)
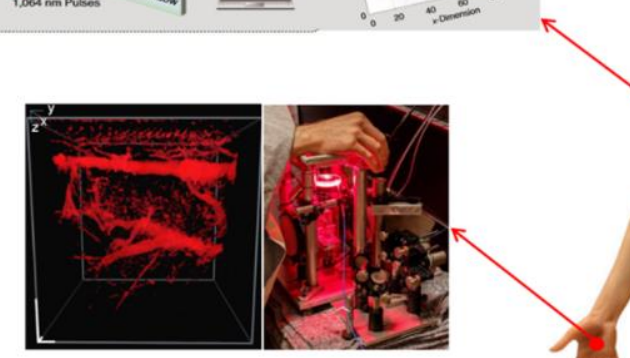

(e)
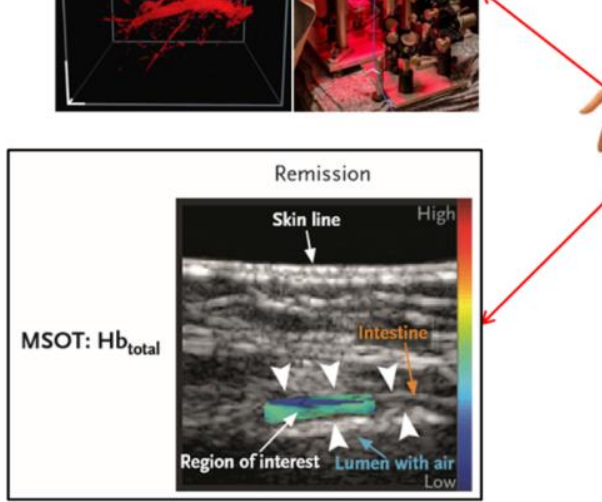

Figure 1-5: Representative clinical PA imaging applications. (a) Overview of the Twente PA mammoscope for breast cancer imaging (left) and a maximum amplitude projection image (right) showing a breast lesion (red). (b) PA imaging of oral vasculature showing blood vessels of the human lip (left), corresponding projection image (middle) and image of a deeper region. (c) 3D PA imaging of palm vessels (left) and the system schematics (right). (d) US/PA imaging of human thyroid (left) and the system schematics (right). Multispectral optoacoustic imaging of (e) inflammatory bowel disease, (f) human carotid artery bifurcation and (g) sentinel lymph node using indocyanine green exogenous contrast agents. Adapted from reference [94].

\subsubsection{The role of the radiofrequency signal}

All applications summarized in Section 1.6.2 rely on the detection of acoustic signals as a result of the thermoelastic conversion of light absorption into pressure waves. Figure 1-6 shows the steps followed for the reconstruction of structural and functional PA images. The temporal acoustic signals are detected using passive ultrasonic detectors, typically operating in the megahertz range to ensure sufficient spatial resolution [116]. Collectively, these signals are referred to as radiofrequency or RF signals. The RF signals collected at a single wavelength are reconstructed to form a PA image (Figure 1-6a). Various image reconstruction algorithms are available depending on the biomedical application, illumination scheme and acoustic detection employed [117]. 


\section{CHAPTER 1}

Irrespective of the reconstruction algorithm used to generate a PA image, the input to each algorithm will always be the time domain PA signal recorded by a device that measures pressure transients, which is typically an ultrasound transducer. The universal back-projection algorithm developed by $\mathrm{Xu}$ and Wang in 2005 has been the most commonly used inversion approach reconstructing the initial PA pressure distribution from a collection of absorbers [118]. This has given rise to multiple types of inversion schemes, including model-based inversion [119]. Additionally, it has been extended to finite sized detection elements through selective-plane detection [120], virtual detector concept [121] and line detector setups [122]. Limited-view geometries arising from instances when the imaged region is not fully accessible are also dealt with reconstruction approaches proposed by $\mathrm{Xu}$ et al in 2004 [123].

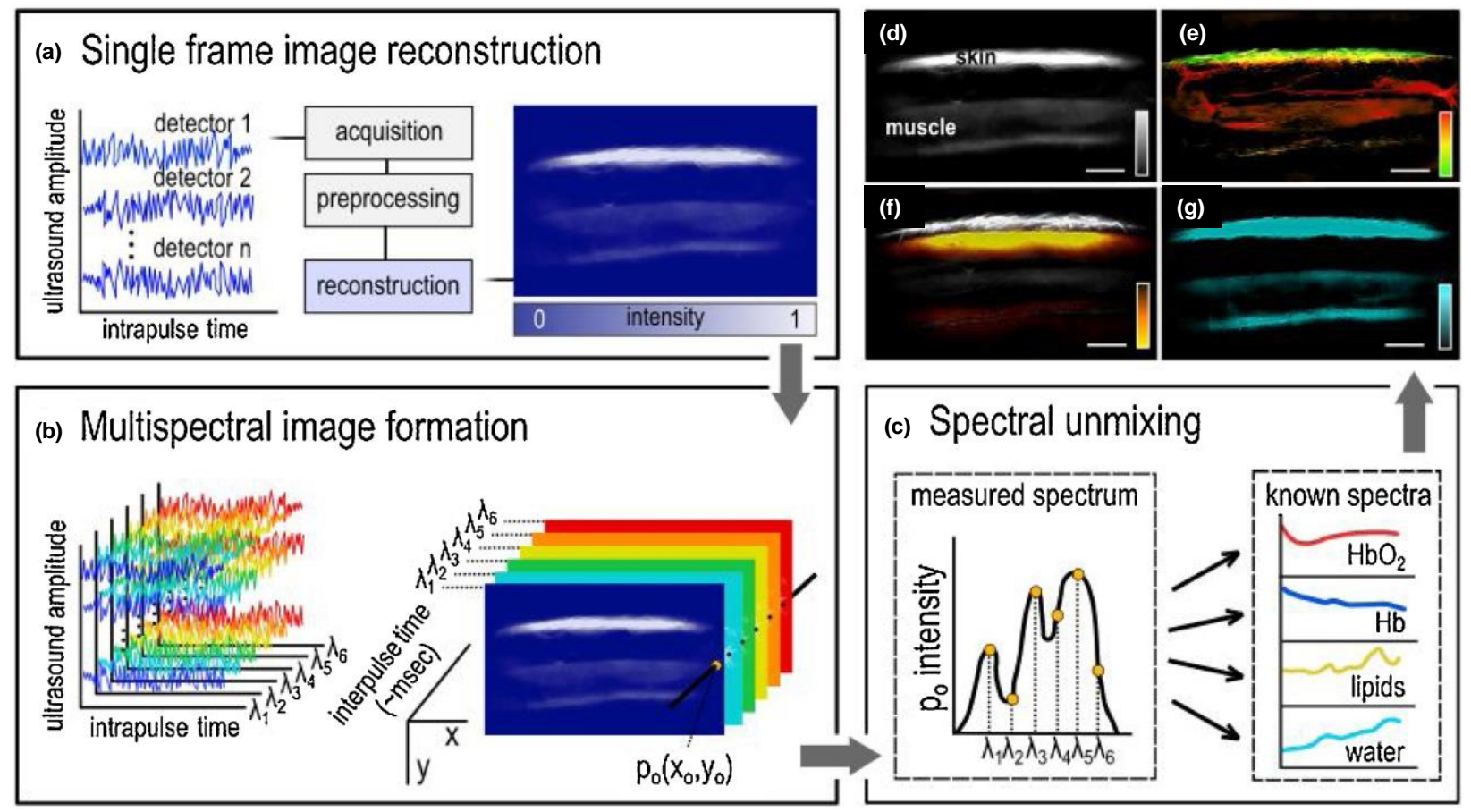

Figure 1-6: Typical reconstruction of PA images. (a) Upon the absorption of a single wavelength $\lambda$, the time-domain ultrasound signals recorded from $n$ acoustic detectors are used to reconstruct a PA image which reveals only structural information. (b) This procedure is then repeated for every $\lambda$ used to illuminate the tissue and each pixel amplitude $p_{o}$ at every location in the image $\left(x_{o}, y_{o}\right)$ is (c) spectrally unmixed using dedicated algorithms from known chromophore spectra. (d) The a single-wavelength grayscale frame obtained at $800 \mathrm{~nm}$ reveals the PA image of the forearm of a healthy volunteer while the spectral unmixing quantifies the content of (e) hemoglobin, (f) lipid and (g) water present. The pseudo color image intensity denotes relative chromophore concentration. The scale bars represent $5 \mathrm{~mm}$ while $x$ and $y$ denote the lateral and axial directions relative to the transducer. Adapted from reference [82]. 


\section{CHAPTER 1}

Single wavelength illumination produces PA images that reveal information about the absorber structure. This often serves as the baseline image used to inform the overall anatomy of the system [94]. In order to obtain functional information regarding the chromophores, multiwavelength illuminations are required in accordance with the optical absorption spectra of the chromophores of interest. The RF signals acquired at each wavelength are used to reconstruct PA images as shown in Figure 1-6b. To recover the relative or absolute concentrations of each chromophores, spectral unmixing is employed on the RF-reconstructed PA image [117]. The per-pixel unmixing is used to calculate the contribution of each chromophore relative to their known optical absorption (Figure 1-6c). This enables the quantification of the spatial distributions of each chromophore of interest such as hemoglobin (Figure 1-6e), lipids (Figure 1-6f) and water (Figure 1-6g) while providing anatomical information (Figure 1-6d).

\subsubsection{Frequency domain analysis of PA signals}

In the aforementioned applications and reconstructions of PA images, only the amplitude of the time-domain signals is utilized. Figure 1-7a shows the theoretical solutions (dashed line) for a fluid layer, a cylinder and a sphere. These temporal waveforms easily differentiate the shapes from one another as the broadband nature of PA signals contains virtually infinite frequencies [124]. However, ultrasonic transducers and their acquisition hardware act as lowpass or bandpass filters, suppressing the high frequency components, altering the PA waveforms. The solid lines of Figure 1-7a show the impact of bandlimiting the PA waveforms, making it difficult to differentiate between objects with different morphologies based only on the time domain RF signals [125]. Sole reliance on the time domain becomes even more challenging during acoustic resolution PA imaging (Figure 1-4c) where numerous PA sources of various sizes, shapes and orientations may be present within the illumination/resolution volume. Their presence results in constructive and destructive interference of the bandlimited temporal PA RF signals [126], leading to the formation of PA speckle.

Generally considered detrimental for resolving individual PA absorbers, the grainy appearance of images that contain speckle obscures boundaries between tissues. Speckle is typically suppressed through image post-processing tools to preserve spatial resolution [127]. In doing so, PA researchers ignore the information that is encoded in the PA RF signals beyond the time domain. 


\section{CHAPTER 1}

However, just like for the US RF signals in QUS, the frequencies of the PA signals carry information on the structure (size, shape and orientation) of the underlying absorber [124], [128], [129]. Figure 1-7b and Figure 1-7c show the time domain PA RF signals from RBCs (at two different orientations relative to the transducer) and a melanoma cell. It is difficult to identify the source of each time domain signal until they are brought into the frequency domain through Fourier transformations (Figure 1-7d). Distinct spectral features in the frequency domain (maxima and minima) are now evident, allowing for differentiation of the cell types and their orientation. The frequency-based analyses methods have been used in high frequency (> $100 \mathrm{MHz}$ ) PA microscopy to characterize biological samples [128], [130], [131] and develop a new imaging mode called frequency or F-mode to study zebrafish vasculature [132].

(a)

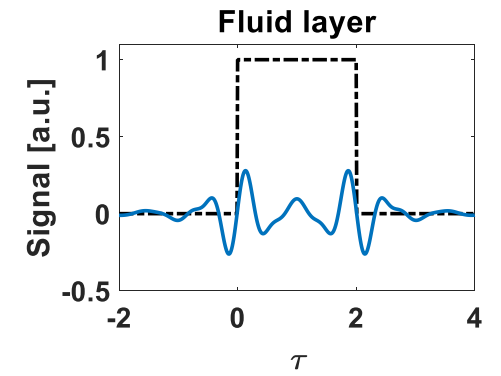

(b)

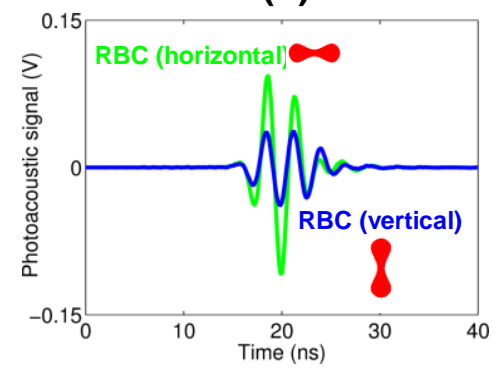

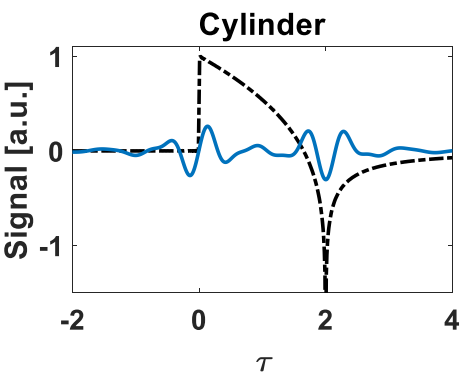

(c)

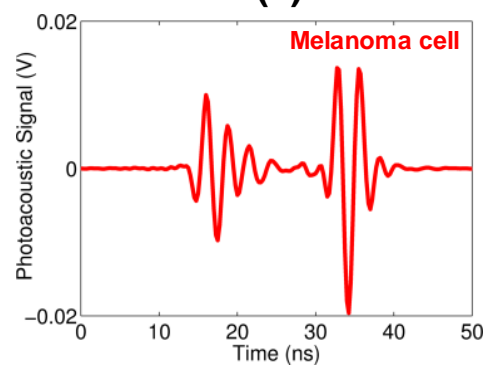

Sphere

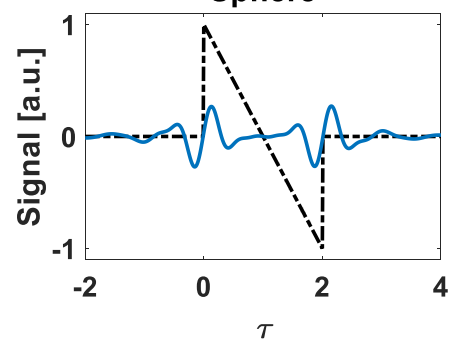

(d)

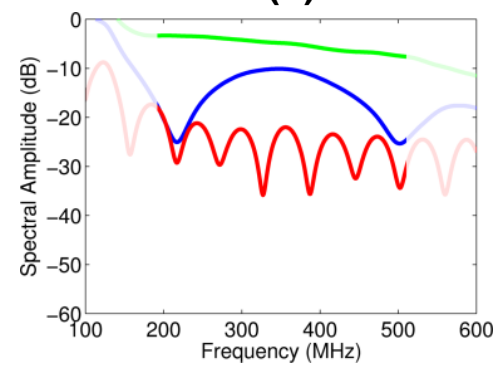

Figure 1-7: (a) Theoretical time domain PA solutions for a fluid layer, cylinder and sphere. The dashed lines show the analytical solutions while the solid lines show the same signals after a bandpass filter (200-500 MHz bandwidth) emulating the transducer response is applied. $\tau$ denotes the dimensionless retarded time (courtesy of Dr. Michael Moore, Ryerson University). Experimentally measured time domain PA signals for (b) red blood cells (RBCs) at two different orientations relative to a $400 \mathrm{MHz}$ single element transducer and (c) a melanoma cell. (d) PA power spectra for the signals shown in (b) and (c). Adapted from reference [130].

\subsubsection{Can PA imaging contribute to treatment monitoring?}

Despite impressive advances, applications of PA imaging to cancer treatment monitoring have been limited to a few feasibility studies (excluding work from this dissertation and published concurrently/after this work) which have focused on the mapping of tumor oxygenation changes 


\section{CHAPTER 1}

post-treatment (assessed through surrogate measurements of oxygen saturation) [133]-[137]. The studies from Bohndiek [134] and Johnson [138] demonstrated the visualization of individual blood vessels with exquisite resolution but were limited to sub-millimeter depths using PA microscopy illumination and detection approaches (Figure 1-8a). The remainder of the studies focused on acoustic-resolution PA imaging without resolving the individual tumor vessels, thus being able to image preclinical tumor models up to $10 \mathrm{~mm}$ in diameter (Figure 1-8b). All studies attempted to quantify the longitudinal, post-treatment relative changes in tumor $\mathrm{sO}_{2}$, hemoglobin levels and PA signal using the only the information provided by the PA signal amplitude. These studies have established the potential of PA imaging for cancer treatment monitoring, particularly due to its ability to study both the anatomical changes in tumor vasculature as well as metabolic/functional changes provided by multiwavelength spectroscopy [94].

\section{(a) Optical resolution PA imaging}

Pretreatment

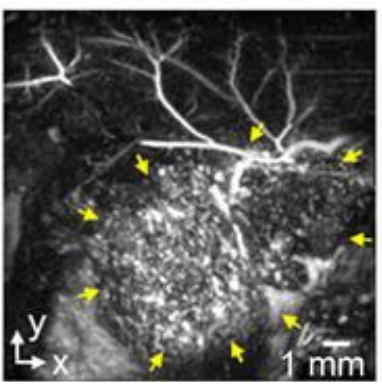

Day 1

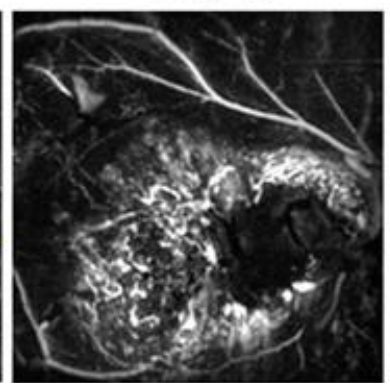

Day 2

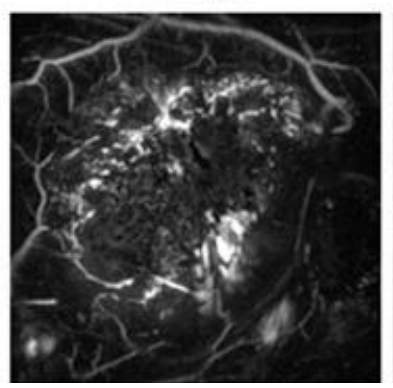

Day 3

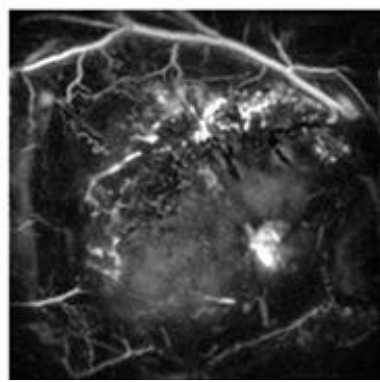

(b) Acoustic resolution PA imaging Baseline

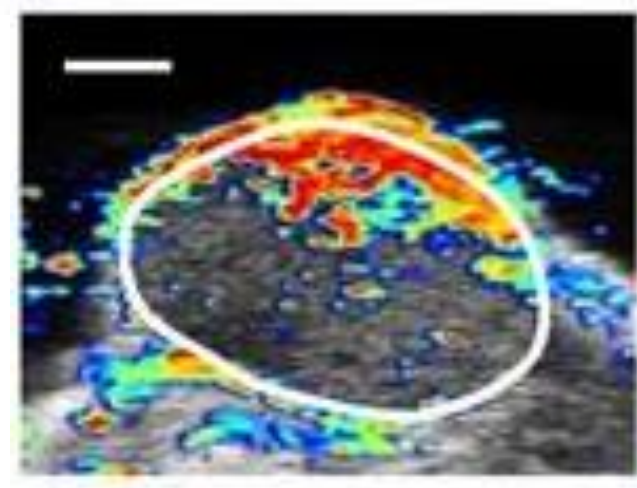
Post 9 Gy

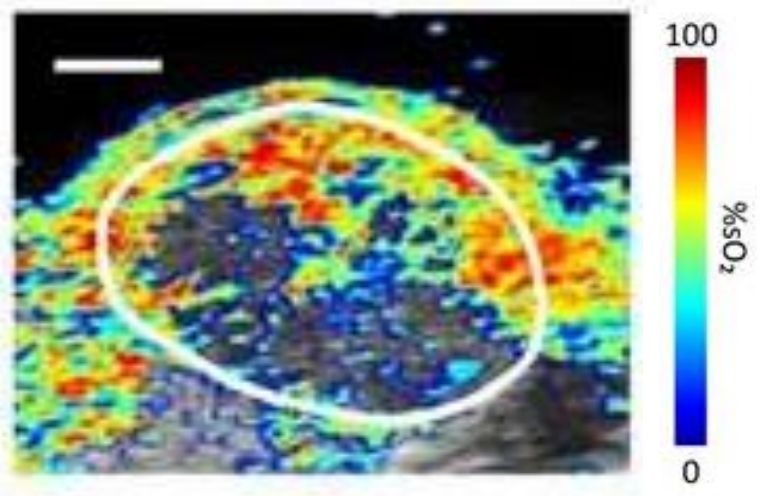

Figure 1-8: Representative examples of optical (top row) and acoustic (bottom row) resolution PA imaging for cancer treatment monitoring. (a) Single wavelength maximum amplitude projection PA images of tumors before and after treatment with a vascular disrupting agent (adapted from reference [138]). (b) Oxygen saturation $\left(\mathrm{sO}_{2}\right)$ images of tumors before and after a single 9 Gy radiation treatment dose. Scale bars denotes $2 \mathrm{~mm}$ (adapted from reference [137]). 


\section{CHAPTER 1}

\subsubsection{Acoustic resolution PA imaging}

Acoustic resolution PA imaging allows for illuminating a larger field of view, flexibility in targeting multiple anatomical sites and achieving more clinically relevant penetration depths [93]. However, as seen in Figure 1-8b, one cannot resolve individual vessels within the tumor but only view the resultant image arising from the superposition of signals from multiple vascular length scales within the field of view [139]. This typically occurs when more than $\sim 10$ absorbers per resolution volume are simultaneously illuminated by a laser pulse and cannot be individually resolved. This is demonstrated in the US and PA imaging of phantoms of Figure 1-9. In this example, the scattering and photoacoustic sources are the same and the images both contain fully developed speckle [140]. The formation of PA speckle is governed by the same physical principles that produce the conventional "grainy" US images of tissue [141].

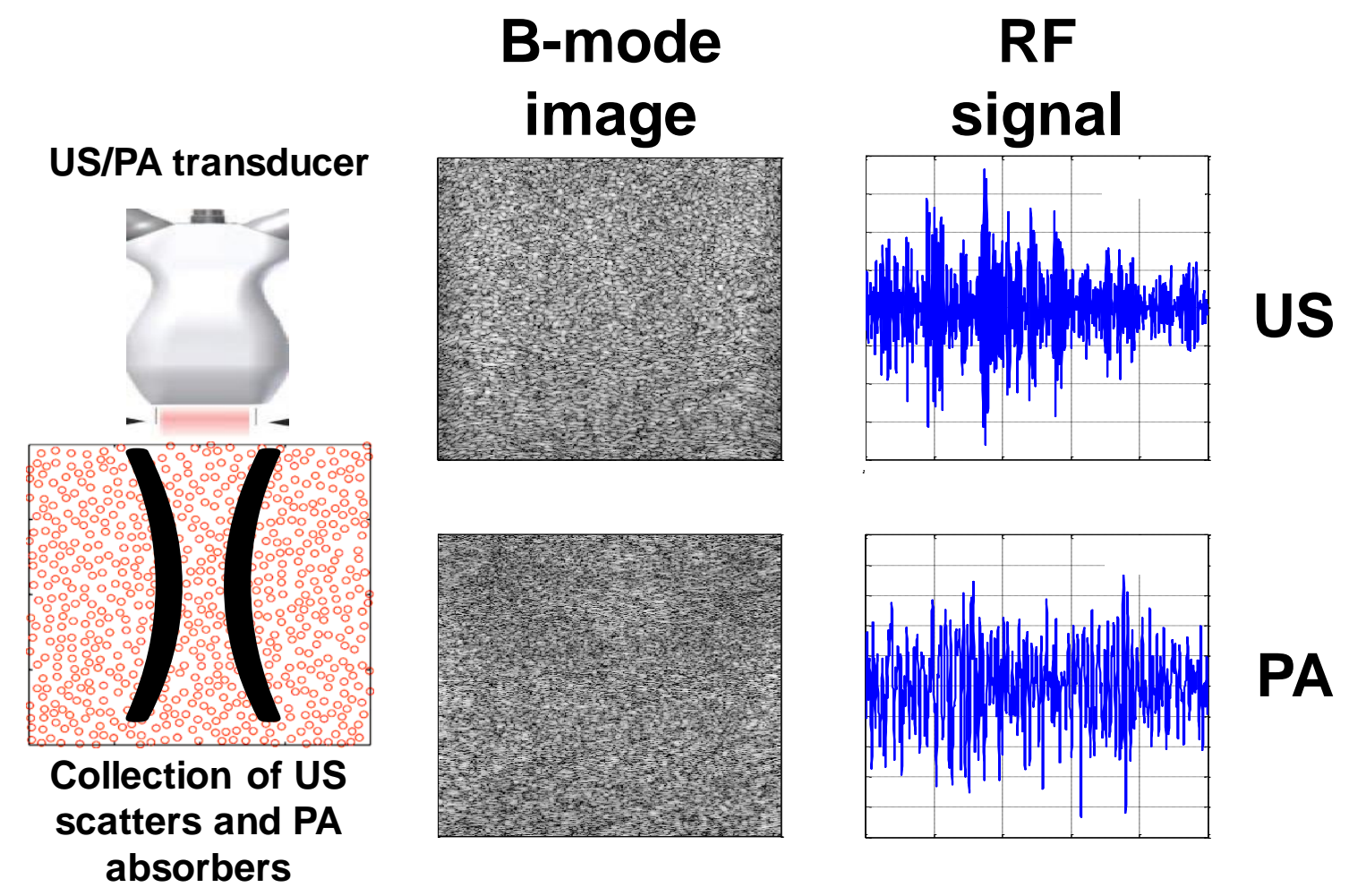

Figure 1-9: US and PA B-mode images and RF signals from a gelatin-based phantom containing spherical carbon beads (1-12 $\mu \mathrm{m}$ diameter). The phantom is imaged with a $40 \mathrm{MHz}$ center frequency linear array illuminating the sample at $680 \mathrm{~nm}$.

The RF signals in both US and PA imaging contain information regarding the shape/size/orientation of the underlying absorbers. This is dictated by the physics of ultrasonic 


\section{CHAPTER 1}

scattering [142], [143] or the physics of PA wave generation [95], [128]. Therefore, analysis of the RF signals in acoustic resolution PA imaging can be used to retrieve properties of the unresolved optical absorbers. In the context of cancer treatment monitoring, the focus of this dissertation, it is possible to combine the functional aspects of PA imaging (based on multiwavelength spectroscopy) with the functional information that would be provided from detailed analysis of the RF signals.

While our group and others have explored the use of RF analysis beyond the time domain for various biomedical applications [95], [144]-[146], this approach has not been used to study vascular changes during cancer treatments. Given the large optical absorption cross-section of RBCs [147], it is reasonable to expect that vascular structures will dominate the PA signal generated from cancerous tumors. In order to develop clinically relevant PA biomarkers of treatment response based on the analysis of the RF signals, the histopathology of tumors must be analyzed to identify the vascular structures giving rise to the PA signals, and how these change with treatment. The latter is crucial in assessing the feasibility of using such biomarkers for quantifying the biophysical changes that occur during cancer treatments. This is particularly important for facilitating the clinical translation of PA imaging so that it can enter mainstream radiology.

\subsection{Thesis hypothesis, aims and organization}

The research presented in this dissertation reports on development of novel biomarkers of cancer treatment response in acoustic resolution photoacoustic imaging. I demonstrate the feasibility of a family of RF-based analysis techniques for identifying and quantifying the early changes to tumor vasculature following treatment. This was shown through simulations of PA absorbers, fractal modeling of tumor vasculature, in vitro tissue mimicking phantoms and in vivo experiments on preclinical models of cancer. The in vivo treatments included a variant of a temperature-sensitive liposome that is currently in clinical trials in addition to an experimental nanobubble-mediated, radiation treatment targeting tumor vasculature. 


\title{
CHAPTER 1
}

\subsubsection{Thesis hypothesis}

The hypothesis guiding this investigation is that photoacoustic imaging biomarkers can quantify the structural and functional vascular changes during cancer treatments that can then be used to monitor cancer therapies.

\subsubsection{Thesis aims}

The aims of this thesis are:

\begin{abstract}
Aim 1: To apply a mathematical formulation for deriving structural and functional parameters that can characterize photoacoustic sources.

Aim 2: To monitor the early therapeutic effect of cancer therapies using biomarkers derived from Aim 1.

Aim 3: To determine if histologically measured metrics of tumor cell death and vasculature correlated with photoacoustic-derived biomarkers of treatment response.
\end{abstract}

\subsubsection{Thesis organization}

The hypothesis and aims of this thesis are addressed in a series of four papers which have either been published (Chapters 2, 3 and 4) or submitted for publication (Chapter 5). The contents of each paper are described in the following sections:

\section{Chapter 2: Insights into photoacoustic speckle and applications in tumor characterization}

In ultrasound imaging, speckle arises from the spatiotemporal superposition of pressure waves backscattered by randomly distributed scatterers. Perhaps due to the dominance of optical resolution photoacoustic microscopy where speckle is suppressed, PA was generally thought to be a speckle-free imaging modality. In this chapter, I present theoretical and experimental evidence for the formation of speckle in acoustic resolution PA imaging. More importantly, I demonstrate through numerical and physical phantoms that PA speckle carries information related to the unresolved absorber structure in a manner similar to US speckle derived from unresolved scattering structures. A fractal-based model of the tumor vasculature was used to study PA speckle from unresolved cylindrical vessels. I show that speckle characteristics and the frequency content 


\section{CHAPTER 1}

of PA signals can be used to monitor changes in average vessel size, linked to tumor growth. These parameters form the theoretical and experiment basis for PA biomarkers that can be used to characterize the unresolved vasculature in acoustic-resolution PA imaging from murine tumors.

\section{Chapter 3: Photoacoustic imaging of cancer treatment response: Early detection of therapeutic effect from thermosensitive liposomes}

The goal of this chapter was to develop a functional biomarker of early treatment response and in the process demonstrate how PA imaging can be used to assess the success of an individualized treatment course, during or immediately following the treatment. The latter has the potential to improve therapeutic outcomes. In this chapter, I used thermosensitive liposomes to deliver chemotherapeutics to a localized tumoral area heated to mild-hyperthermia. The doxorubicin inside the liposomes was burst released into the tumor vasculature, inducing hemorrhaging, blood coagulation and vascular shut down. To probe the effects of this treatment, I developed an oxygen saturation algorithm based on the amplitude of the PA signals at two wavelengths. This histogrambased approach to quantify the distribution of oxygenation across the entire tumor volume as well as longitudinally following treatment (from 30 minutes to 7 days post-treatment). The PA functional biomarker developed in this chapter could be used to monitor the effects of the temperature sensitive liposome by measuring the oxygenation drop as a function of time posttreatment. The latter led to tumor regression in $90 \%$ of the cases. The changes in tumor oxygenation (as assessed from the oxygen saturation values) were further corroborated with histological data using vascular perfusion markers. These results allowed me to propose a plausible mechanism of the cellular events taking place in the chemotherapeutic-treated tumor regions over the first 24 hours post-treatment. The work shown here demonstrates for the first time the potential of PA-derived oxygenation biomarkers as a surrogate prognostic marker for predicting the therapeutic outcome of a vascular targeting cancer treatment.

\section{Chapter 4: Photoacoustic signal characterization of cancer response: Correlation with changes in tumor oxygenation}

In Chapter 4, I develop a new imaging biomarker of cancer response which is based on the frequency analysis of the PA RF signals. Building on the findings of Chapter 2, I tested whether

the spectral slope of the normalized PA power spectra acquired at various timepoints post 


\section{CHAPTER 1}

thermosensitive liposome treatments changes as a function of time. This parameter showed sensitivity to absorber size in numerical simulations, in vitro phantoms and in vivo tumor vasculature. Most importantly, in combination with the tumor oxygenation biomarkers (i.e. oxygen saturation) developed in Chapter 3, treatment responders were identified as early as 30 minutes post-treatment. It was shown that the spectral slope parameter correlated with treatment-induced hemorrhaging, which we postulate increased the optical absorber effective size via interstitial red blood cell leakage. Combining frequency analysis and oxygen saturation estimates differentiated treatment responders from non-responders/control animals by probing the treatment-induced structural changes of blood vessels.

\section{Chapter 5: Photoacoustic imaging biomarkers for monitoring biophysical changes during nanobubble-mediated radiation treatment}

In this chapter, I explore the feasibility of using PA biomarkers to quantify therapeutic response to a novel nanobubble-mediated radiation treatment. This was done using new class of submicron, vascular disrupting, ultrasonically simulated nanobubbles which were used to test whether they could enhance radiation therapy. In vivo experiments were conducted on mice bearing prostate cancer tumors. Nanobubble and radiation treatments were compared against conventional microbubbles and radiation alone. Histology provided metrics of tumor vascularity and tumoral cell death, both of which were compared to PA-derived biomarkers (structural and functional). PA metrics of oxygen saturation reveal a $20 \%$ reduction within 24 hours post-treatment and that the spectral slope could separate the tissue response to the nanobubble treatments from the microbubble counterparts. Histopathological assessment was found to correlate well with PA biomarkers of treatment response, demonstrating their practicality in PA imaging for studying the biophysical changes during cancer treatments.

Finally, in Chapter 6 of this thesis I will provide an overview and summary of the findings and conclusions of Chapters 2-5. I will also address the limitations of this work and provide a roadmap for future studies on PA of cancer treatment monitoring. The thesis Appendix provides the journalspecific permissions to reproduce copyrighted published materials. 


\section{CHAPTER 2}

\section{Insights into photoacoustic speckle and applications in tumor characterization}

The contents of this chapter have been previously published in Photoacoustics. Permission to reproduce this article was granted by Elsevier and is provided in Appendix A.1.1.

E Hysi, MN Fadhel", MJ Moore*, J Zalev, EM Strohm and MC Kolios. "Insights into photoacoustic speckle and applications in tumor characterization" Photoacoustics 2019; 14 37-48 https://doi.org/10.1016/j.pacs.2019.02.002

*These authors contributed equally to this work.

\subsection{Introduction}

Photoacoustic (PA) imaging allows for high contrast visualization of the vasculature [83], pharmacokinetic drug distribution [148] and neuronal functional connectivity [101]. Clinical applications include melanoma detection [149], assessment of Crohn's disease [113], breast imaging [106] and cancer treatment monitoring [133], [150]-[153]. PA images are affected by the light illumination geometry, choice of optical wavelength and ultrasonic detection characteristics. Speckle is a ubiquitous property of all coherent modalities such as laser imaging [154], ultrasound (US) [155] and optical coherence tomography [46]. Due to the similarities between the two techniques, PA speckle is analogous to US speckle, which results from the spatiotemporal superposition of waves backscattered from randomly positioned objects within the imaging transducer's resolution volume. At clinical frequencies, ultrasound speckle is considered fully developed for a scatter density of $>100 / \mathrm{mm}^{3}$ or, more generally, when there are at least 10 scatterers per transducer resolution volume [156]. In US, acoustic impedance mismatches from sub-resolution inclusions scatter the incident US pulse. The scatterer' spatial position gives rise to phase differences which produce fluctuations in image intensity (i.e. speckle) due to wave superposition. Such patterns are deterministic and described by first and second-order statistics. As long as the position of the scattering sources does not change with time, the speckle patterns remains unchanged. Therefore, speckle encodes spatial information about the scatterers. The distribution of signal amplitudes (first-order statistics) is independent of the transducer aperture [157]. Second-order statistics, e.g. the spatial autocovariance function (ACVF), provide an estimate of speckle size, which in turns depends on pulse bandwidth, beamwidth, transducer fnumber, working distance and consequently, the spatial resolution of the US system [158]. 


\section{CHAPTER 2}

The physics of PA acoustic wave propagation is the same as US wave propagation. The main difference between the signals produced by both modalities lies in the broadband nature of PA imaging. Theoretically, PA signals are infinitely broadband [124] while the frequency content of backscattered US signals is only a subset of the frequencies emitted in the initial transmit pulse from the transducer [140]. While PA signals are also bandpassed by the receiving transducer, they contain a larger range of frequencies than in the case of US signals. This might be desirable in approaches involving frequency analysis of PA signals and images such as F-Mode [159] or radiofrequency spectroscopy [95]. For this reason, frequency-dependent attenuation, diffraction

and the transducer focus, geometry, frequency and bandwidth affect PA images [160]. PA pressure waves from multiple absorbers also generate constructive and destructive interference [126]. At boundaries between media, constructive interference typically produces a high amplitude signal, while both constructive and destructive interference occurs between boundaries. The signal from the boundary usually saturates the dynamic range of the resultant PA images giving the impression that acoustic resolution PA imaging is a speckle-free modality [127], [161], [162] even though speckle is present in applications involving non-resolvable absorbers [144], [163]. The prominence of this boundary "edge-effect" is strongly influenced by the transducer properties, system response and absorber concentration. Our group has demonstrated through simulations [126], [164] and experiments [145], [165], [166] that the signals giving rise to speckle are dependent on the reconstruction approach, spatial distribution of PA sources and the bandwidth of the ultrasound detector.

In US imaging, the "grainy" appearance of speckle makes it difficult to differentiate between different types of soft tissues which suffer from low contrast due to the narrow range in their mechanical properties. To overcome this limitation, quantitative ultrasound (QUS) has emerged as a system-independent approach relying on the analysis of the radiofrequency (RF) signals from the backscattered US waves [30]. QUS has been used to quantify scatterer shape, size, concentration, spatial organization or mechanical properties [167] and characterize blood [168] or breast cancer treatment response [169]. Given the parallels between US and PA imaging, it is hypothesized that the similar analysis techniques can be applied to extract quantitative parameters in PA without having to resolve the individual absorbing structures. 


\section{CHAPTER 2}

PA speckle has been largely considered to be a detrimental aspect of imaging and most illumination and detection geometries as well as image post-processing tools attempt to suppress its formation [83]. However, in cases where high resolution PA images cannot be attained, and speckle is present, the deterministic nature of speckle permits quantitative analysis of the underlying absorbers. In this work, we demonstrate the formation of PA speckle in limited-view transducer geometries and demonstrate how speckle patterns are affected by the imaging transducer properties. Theoretical and experimental evidence shows that PA speckle encodes structural information about non-resolvable absorbers. Lastly, we illustrate how tumor vascular development can be monitored in cases where PA speckle is present in acoustic-resolution PA imaging. In this paper, we establish the mathematical formulation of PA speckle in limited-view transducer geometries and demonstrate experimentally how speckle patterns are affected by imaging transducer properties. We also discuss how the structural information encoded in PA signals can be extracted by RF analysis of signals in the temporal and frequency domain and how this approach can be applied to monitor tumor vascular development.

\subsection{Mathematical formulation of PA speckle}

PA speckle arises from the linear superposition of the pressure waves generated as a result of the short-pulse, optical excitation of a medium consisting of optical absorbers. Figure 2-1 shows a visual representation of PA speckle formation from a collection of randomly positioned absorbers (Figure 2-1a) imaged with a linear array transducer with $N$ elements. The mathematical formation of PA speckle begins with derivations of the time-dependent PA emission obtained by solving the wave equation for pressure $p$ [124], [139], [170]. Using Green's functions for spherical and cylindrical absorbers, the solution for the wave equation can be represented in terms of a velocity potential $\phi$ :

$$
\phi(\boldsymbol{r}, t)=-\frac{\beta}{4 \pi \rho C_{P}} \int_{0}^{t} d t^{\prime} \int g\left(\boldsymbol{r}, t \mid \boldsymbol{r}^{\prime}, t^{\prime}\right) H\left(\boldsymbol{r}^{\prime}, t^{\prime}\right) d \boldsymbol{r}^{\prime}
$$

where, $\beta$ is the thermal expansion coefficient, $\rho$ is the density, $C_{P}$ is the heat capacity per unit mass, $r$ is the spatial position, $H$ is the heating function defined as the energy per unit volume and time $t$ deposited by the incident radiation beam in the sample. The pressure is the first time 


\section{CHAPTER 2}

derivative of the velocity potential, namely $p=-\rho \partial \phi / \partial t$ (Figure 2-1b). In our model, the time domain impulse response of the transducer as a function of center frequency is given by [164]:

$$
g_{T}\left(\omega_{0}, \xi, t\right)=\frac{\xi}{\sqrt{2 \pi}} \exp \left(-\frac{\xi^{2} t^{2}}{2}\right) \cos \left(\omega_{0} t\right)
$$

where, $\varpi_{0}$ is the center frequency, $\xi$ is the $-6 \mathrm{~dB}$ bandwidth. To account for the limited-view geometry, a directivity function $D(\alpha)$ was computed for each element as a function of angle $\alpha$ relative to the center of each transducer element [171]:

$$
D(\alpha)=\left|\frac{2 \mathcal{J}_{1}(k R \sin \alpha)}{k R \sin \alpha}\right|
$$

where, $\mathcal{J}_{1}$ is the first order Bessel function, $k$ is the wavenumber and $R$ is the transducer aperture. The bandlimited (BL) PA signal from $M$ absorbers (Figure 2-1c) is the temporal convolution of the directivity-corrected pressure from each absorber $p_{m}(\boldsymbol{r}, t)$ with Eq. (2.2):

$$
p_{B L}(t)=\sum_{m}^{M}\left[p_{m}(\boldsymbol{r}, t) \times D(\theta)\right] * g_{T}\left(\omega_{0}, \xi, t\right)
$$

In acoustic-resolution PA imaging using linear array transducers, the image is generated from the recorded PA signals using delay-and-sum beamforming by dynamically focusing to a point in the imaging space using $N_{A}$ sub-aperture elements. The $p_{n}^{B L}(t)$ generated from an absorbing source located at location $\boldsymbol{r}_{m}$ will arrive at the $n^{\text {th }}$ transducer element at time $t=\left(\left|\boldsymbol{r}_{n}-\boldsymbol{r}_{m}\right|\right) / v_{s}$. At the $(n+1)^{t h}$ element, the RF signal from the same source will be delayed by $t=\left(\left|\boldsymbol{r}_{n+1}-\boldsymbol{r}_{m}\right|-\right.$ $\left.\left|\boldsymbol{r}_{n}-\boldsymbol{r}_{m}\right|\right) / v_{s}$ due to the path difference created by the spatial separation of adjacent elements. Signals received from the spatial position $\boldsymbol{r}_{m}$ were compensated for the relative delays on subaperture $N_{A}$ and adding their contributions. The beamformed (BF), bandlimited PA RF line (Figure 2-1d) then becomes:

$$
p_{n}^{B L}(t)=\sum_{n=0}^{N_{A}-1} p_{n}^{B L}\left(t-\tau_{n}\right)
$$




\section{CHAPTER 2}

The summation/interference of all time-delayed PA signals is the source of PA speckle, as is the case in US imaging [155]. The phase of each wavelet is determined by the time delay, and thus spatial position, of each absorber within the transducer's resolution volume. The summation of the wavelets contributes to an interference pattern. The interference pattern depends on the spatial distribution and physical properties of the absorbers and can be analyzed using speckle envelope statistics [172] and frequency-based techniques [169], as described in the next section. Following beamforming, the PA images are displayed using the logarithmically compressed Hilbert transform of the RF matrix at each scanning location. The final, reconstructed image shown in (Figure 2-1e) is derived by taking the envelope of the RF signals using the Hilbert transform $\mathcal{H}$ :

$$
I(x, z)=20 \log _{10}(A)=20 \log _{10}\left|\mathcal{H}\left\{p_{B L}^{B F}(x, z)\right\}\right|
$$

The spatial coordinates $(x, z)$ are obtained using the transducer pitch and speed of sound in the lateral $(x)$ and axial $(z)$ directions, respectively.

(a)

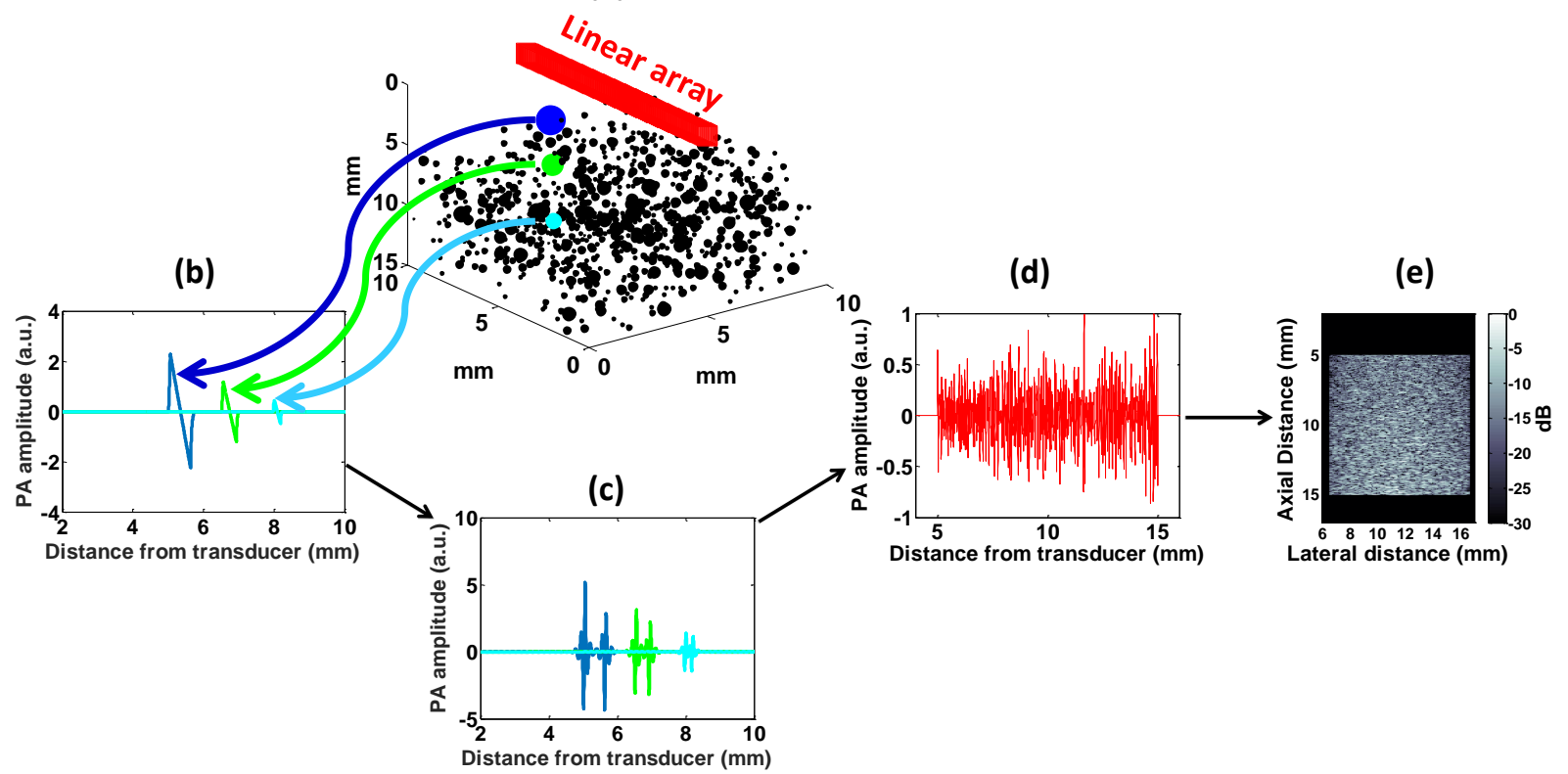

Figure 2-1: Formation of PA speckle. (a) 3D visualization of the geometry of a numerical phantom containing randomly positioned spherical absorbers of different diameter. (b) Typical nonbandlimited PA signals from absorbers of three different diameters. (c) Bandlimited version of PA signals shown in (b). The range of frequencies is determined by the ultrasound detector used. (d) Representative, beamformed PA RF line with contributions from all absorbers. (e) Resultant PA B-mode image obtained by delay-and-sum beamforming. 


\section{CHAPTER 2}

\subsection{Materials and methods}

In this section, three analysis techniques will be introduced for probing the morphological properties of non-resolvable absorbing structures giving rise to speckle. Simulations of media containing spherical and cylindrical absorbers will be used to demonstrate the formation of PA speckle. Experimental phantoms containing non-resolvable absorbers will illustrate the effect of the imaging transducer spatial resolution on speckle size. As the primary source of the photoacoustic signals in tissues is red blood cells, simulations of vascular trees and PA images of mouse tumors will be used to illustrate changes in speckle characteristics in growing tumors.

\subsubsection{Analysis methods}

Figure 2-2 shows an overview of the three techniques used in this paper for analyzing PA signals. Each method was applied to both simulations and experimentally acquired PA images containing speckle from spherical and cylindrical absorbers. The major distinction between the techniques was whether the signal analysis was performed in the temporal or frequency domain. In combination, these techniques will be used to evaluate the changes in PA images due to variations in the concentration and size of unresolvable absorbers (Sections 2.4.1.1 and 2.4.1.2) and identify periodic patterns present in PA images containing speckle (Section 2.4.1.3). Each analysis technique is described below:

(i) The envelope statistics method models the amplitude of the envelope of the RF signals by a statistical distribution. The distribution characteristics depend on the physical size, concentration, and spatial distribution of absorbing structures [172]. The amplitude of the envelope of the RF signals, $A$, for each RF signal in a phantom was compared to three statistical distributions: Rayleigh, Generalized Gamma, and Nakagami (Figure 2-2b). Fully developed speckle was established when the ratio of the mean to the variance of the amplitude (also known as the speckle signal to noise ratio, SNR) is equal to 1.91 [173]. The fit parameters of each distribution were recorded as a function of absorber size and concentration. 


\section{CHAPTER 2}

(ii) The radiofrequency spectroscopy technique is based on the analysis of the frequency content of the PA signals which changes based on the size and shape of the absorbing structures [124]. The spectral slope (SS) of the normalized or non-bandlimited power spectrum (Figure 2-2c, middle box) was computed by linear regression within the $-6 \mathrm{~dB}$ bandwidth of the transducer for a range of absorber sizes and increasing absorber size polydispersity. The normalized PA power spectra were obtained either from the nonbandlimited PA frequency spectra in the case of the simulations, or by normalizing the power spectra by spectra acquired from a reference signal for experiments. This approach is similar to what is used in ultrasound tissue characterization [29]. A straight line was fit to the resultant normalized spectra within the $-6 \mathrm{~dB}$ bandwidth of the transducer in order to extract the spectral parameters:

$$
P S_{f i t}(f)=S S \times f+Y_{i n t}
$$

where, $P S_{f i t}$ is the result of performing linear regression on the normalized spectra, $S S$ is the spectral slope measured in $\mathrm{dB} / \mathrm{MHz}$ and $Y_{\text {int }}$ is the y-intercept of the fit measured in $\mathrm{dB}$. The average SS was computed for all the spectra included in a ROI, for various of absorber sizes and increasing absorber size polydispersity.

(iii) The cepstral analysis technique uses the frequency content of the RF signals to estimate the absorber spacing [174]. This technique determines the temporal location of the dominant (first) cepstral peak which can be converted to a physical distance by accounting for the speed of sound within the medium, $1540 \mathrm{~m} / \mathrm{s}$ in this case (Figure 2-2c, bottom box). The Euclidean norm was used to calculate the true absorber spacing from spatial coordinates. 
(a)

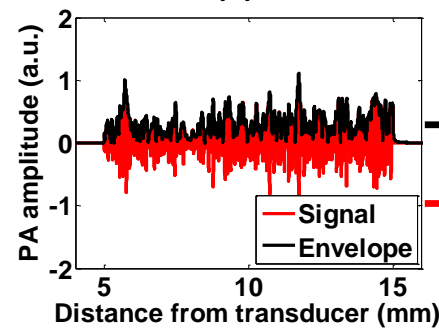

(c)

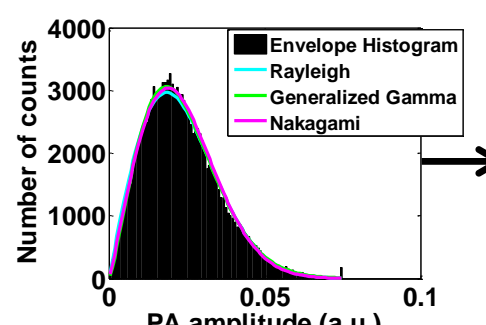

PA amplitude (a.u.)

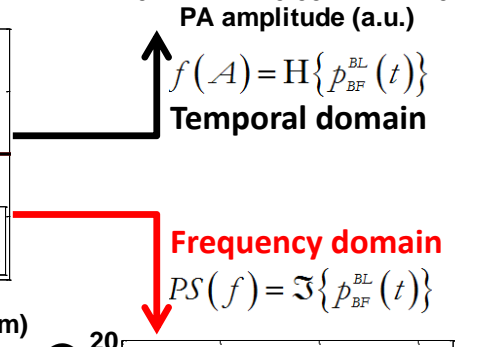
Distance from transducer $(\mathrm{mm})$

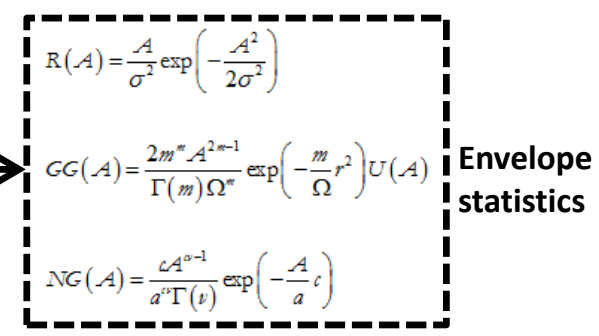

(1)
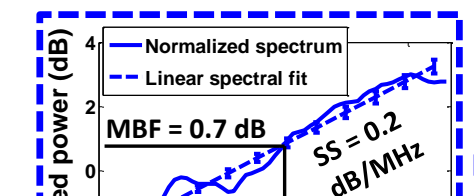
Radiofrequency spectroscopy

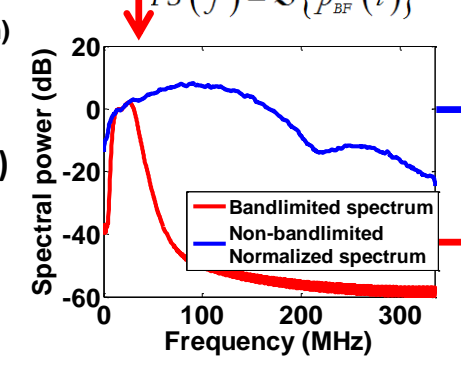

Figure 2-2: Overview of analysis techniques. (a) Representative PA RF signal (red) and its envelope (black). (b) Temporal domain analysis obtained through envelope statistics (top box). (c) Frequency domain analysis obtained through the Fourier transform of PA signals yields radiofrequency spectroscopy (middle box) and cepstral analysis (bottom box).

For each technique, the PA B-mode images obtained from Eq. (2.6) were divided into overlapping (75\%), square-shaped ROIs whose size was $\sim 10$ ultrasonic wavelengths (estimated from the center frequency of the imaging transducer). The average size of the speckle in a PA B-mode image was calculated by the 2-D ACVF which is a descriptor of the spatial correlation of texture [158]. In the frequency domain, the ACVF is given by:

$$
\operatorname{ACVF}\left(\omega_{x}, \omega_{z}\right)=U\left(\omega_{x}, \omega_{z}\right) U^{*}\left(\omega_{x}, \omega_{z}\right)=\left|U\left(\omega_{x}, \omega_{z}\right)\right|^{2}
$$

where, $U\left(\omega_{x}, \omega_{z}\right)$ is the 2-D Discrete Fourier transform of the PA image obtained from Eq. (2.6) which contained within the ROI over the spatial frequencies $\omega_{x}$ and $\omega_{z}$, and $U^{*}\left(\omega_{x}, \omega_{z}\right)$ is its complex conjugate. After transforming the $\operatorname{ACVF}\left(\omega_{x}, \omega_{z}\right)$ back to the spatial domain through an 


\section{CHAPTER 2}

inverse Fourier transform, the cross-sections of the corresponding 2-D ACVF in the axial and lateral directions is taken to calculate the respective speckle sizes.

\subsubsection{Simulations}

\subsubsection{Modeling spherical absorbers}

Numerical phantoms of dimensions $10 \times 10 \times 10 \mathrm{~mm}$ were constructed within a $1000 \mathrm{~mm}^{3}$ region of interest (ROI). Spherical beads of radius $a$ were randomly positioned within the phantom. PA signals were computed from the time derivative of Eq. (2.1). The transducer modeled was the VevoLAZR (Fujifilm-VisualSonics Inc., Toronto, Canada) which is a $21 \mathrm{MHz}$ linear array probe (LZ250 model, 256 elements, $90 \mu \mathrm{m}$ pitch, 9-33 MHz -6dB bandwidth, $5.44 \times 10^{6} \mu \mathrm{m}^{3}$ resolution volume) [151], [175], [176]. It was positioned $5 \mathrm{~mm}$ above the phantom. A total of 86 distinct, numerical phantoms were simulated to test analysis techniques described in Section 2.3.3:

(i) The envelope statistics phantoms (27 phantoms) were simulated by changing the size (5$60 \mu \mathrm{m})$ and the concentration of beads $(0.1,1$ and 10 beads per resolution volume).

(ii) The radiofrequency spectroscopy phantoms (50 phantoms) were simulated by changing the bead radius from 5 to $135 \mu \mathrm{m}$ to test the effect of size and polydispersity (achieved by changing the standard deviation ( $\theta$ parameter) of a Generalized Gamma distribution for bead size).

(iii) The cepstrum analysis phantoms (11 phantoms) were simulated with different populations of periodic and random absorbing structure (defined as the grid-to-random or G:R ratio). The beads were either placed in a 3D grid of defined spacing (100:0\% ratio) or partially randomized (50:50\% and 15:85\% ratios). For the latter, the beads concentration ranged from 8 to $1469 / \mathrm{mm}^{3}$.

\subsubsection{Modeling cylindrical absorbers}

A 3D computational vascular network model was generated to investigate the impact of the geometrical arrangement of cylindrical absorbers (blood vessels) on the formation of PA speckle. 


\section{CHAPTER 2}

The approach is based on a fractal geometrical model described elsewhere [139], [177], [178]. Briefly, the network begins with a parent vessel bifurcating to give rise to two daughter branches which bifurcate further. Using cylindrical segments, the vascular trees evolved successively down to the arteriole level (diameter $\sim 20 \mu \mathrm{m}$ ). For the purposes of this study, chaotic tumor vasculature [179] was modeled by randomly changing the branching angle between $25-140^{\circ}$ [178]. It was not our intention to model the complex vascular morphology of a tumor but rather to investigate the effects that increasing the vessel diameter, branching order and vessel length might have on PA speckle signals. These changes in vessel characteristics are well documented in animal models of tumor growth [180], [181].

The simulation parameters were based on histological measurements of vessel size from in-vivo mouse tumors at 7 and 14 days post-inoculation (sees Section 2.3.3.2) [150], [151]. The following simulation parameters were used to achieve a mean diameter of all cylinders as close to the experimentally measured vessel size:

(i) Day 7 tumors: $184 \mu \mathrm{m}$ parent vessel diameter; $0.75 \mathrm{~mm}$ parent vessel length; $37.8 \mu \mathrm{m}$ mean diameter; $360 \mu \mathrm{m}$ mean vessel length; branching order of 9; 508 total vessels.

(ii) Day 14 tumors: $460 \mu \mathrm{m}$ parent vessel diameter; $1 \mathrm{~mm}$ parent vessel length; $48.2 \mu \mathrm{m}$ mean diameter; $352 \mu \mathrm{m}$ mean vessel length; branching order of 12; 4092 total vessels.

For every cylinder in the vascular tree, the PA signals were computed by solving Eq. (2.1) for the radii $r$ and lengths $L$ listed above. For these simulations, the transducer modeled was a VevoLAZR $40 \mathrm{MHz}$ linear array probe (LZ550 model, 256 elements, $50 \mu \mathrm{m}$ pitch, 9-50 MHz -6 dB bandwidth).

\subsubsection{Experiments}

\subsubsection{Construction and imaging of physical phantoms}

To study the dependence of PA speckle dimensions on the properties of the imaging transducer, three physical phantoms were constructed and imaged with four transducers $(5-400 \mathrm{MHz}) \mathrm{using}$ three different PA imaging systems, the Ultrasonix RP linear array, the VevoLAZR US/PA linear 


\section{CHAPTER 2}

array, and the SASAM PA microscope (Supplementary Figure 2.A). The dimension of the phantoms, the acoustic properties of the imaging transducers and the phantom preparation methods are summarized in Supplementary Figure 2.B. The optical absorbers for each system were either black glass (5 MHz transducer) or black polystyrene (40/200/400 MHz transducer) beads. The phantoms were constructed so that there were at least 10 beads per resolution volume of each transducer. For images acquired using the 5, 40, and 200/400 MHz transducers, the speckle size was estimated by computing a single ACVF for 305, 315, and 120 different overlapping ROIs within the image, respectively. All ACVFs from the same image were then averaged to obtain the mean speckle size. For each phantom, envelope statistics techniques were applied to obtain the SNR and the Nakagami $m$ parameter. For the $40 \mathrm{MHz}$ transducer, each of the analysis techniques described in Section 2.3.1 were performed for two different spherical absorber diameters (3.5 and $15 \mu \mathrm{m})$.

\subsubsection{In-vivo imaging of tumors}

All experimental protocols were approved by the University Health Network (Toronto, Canada) Animal Care Committee. A murine breast cancer cell line (EMT-6) was inoculated subcutaneously on the footpad of BALB/c mice. Volumetric PA images of the tumors were acquired using $750 \mathrm{~nm}$ illumination with the VevoLAZR system (Fujifilm-VisualSonics, Toronto, Canada). Imaging was performed 7 days $(n=3$ mice) and 14 days $(n=4$ mice) post-inoculation with mice sacrificed after imaging. CD31 histological staining was used to compute the average vessel size at each imaging time point for comparisons with the vascular trees (see Section 2.3.2.2). A PA reference phantom consisting of black carbon beads (1-12 $\mu \mathrm{m}$ diameter) was used as a measure of the system dependencies and to remove the system response for RF spectroscopy [151], as described in Section 2.3.1. The size of speckle (estimated from the 2D ACVF) from images of in-vivo tumors as well as simulated vascular trees were compared to the transducer spatial resolution. 


\section{CHAPTER 2}

\subsection{Results and discussion}

\subsubsection{Probing the structural properties of non-resolvable, spherical absorbers}

\subsubsection{Envelope statistics}

Figure 2-3a shows PA B-mode images from collections of randomly positioned, spherical absorbers for increasing bead concentration. The images are normalized to the maximum image pixel amplitude of the image containing approximately 10 beads per resolution volume (b/rv) of the imaging transducer $\left(5.44 \times 10^{6} \mu \mathrm{m}^{3}\right)$. As absorber concentration increased, the PA signal amplitude increased, resulting in a shift of the envelope histograms to the right (Figure 2-3b). The envelope histograms can be used to assess the presence of fully developed speckle by relying on the either SNR metric and/or the fit to the Rayleigh probability density function (PDF) [172]. Fully developed speckle was observed as the speckle SNR approached the value of 1.91 with increasing bead concentration (Figure 2-3b). The other two statistical models, the Generalized Gamma and Nakagami provide better fits than Rayleigh in cases of lower number densities $(0.1$ and $1 \mathrm{~b} / \mathrm{rv})$ and become identical to Rayleigh for $\geq 10 \mathrm{~b} / \mathrm{rv}(\mathrm{SNR}=1.91)$. These results agree well with those obtained with envelope statistical analysis in US imaging [182] and support the assertion that speckle statistics can be used to characterize acoustic-resolution PA images. Studies from US imaging show that the Generalized Gamma $a$ parameter is sensitive to scatterer size and concentration. Additionally, the Nakagami $m$ parameter approaches 1 for fully developed speckle, independent of scatterer size [183]. Our simulations demonstrate that the same trend holds when considering PA emission from uniformly illuminated spheres. Specifically, the a parameter increases monotonically with absorber radius (Figure 2-3c) and the $m$ parameter becomes 1 for $>100$ absorbers $/ \mathrm{mm}^{3}$ (Figure 2-3d). The $\Omega$ parameter (Figure 2-3e) increases 36x for the range of number densities examined. In US imaging, the Nakagami fit parameters have been used to characterize the scatter properties in malignant and benign breast tumors [183]. We hypothesize that application of similar statistical analysis techniques to experimentally acquired PA data can be used for characterizing changes during vascular-targeted cancer treatments [65]. 
(a)

$0.1 \mathrm{~b} / \mathrm{rv}$

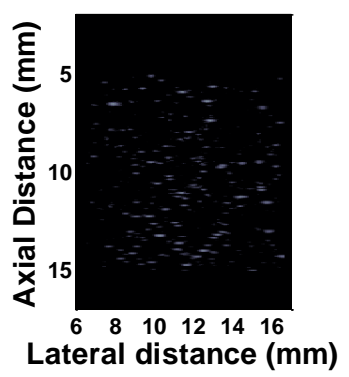

(b)

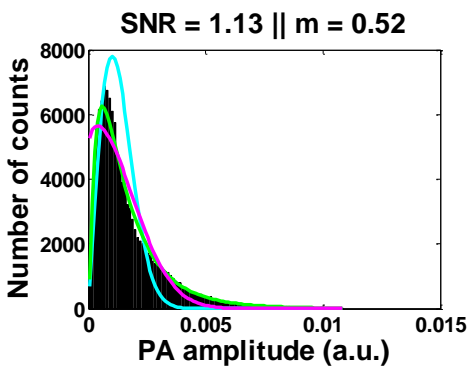

(c)

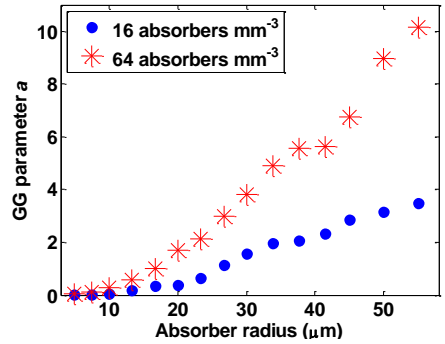

$1 \mathrm{~b} / \mathrm{rv}$
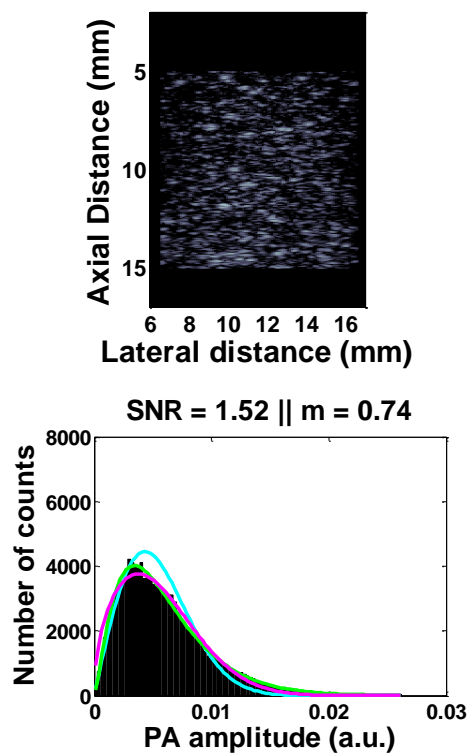

(d)

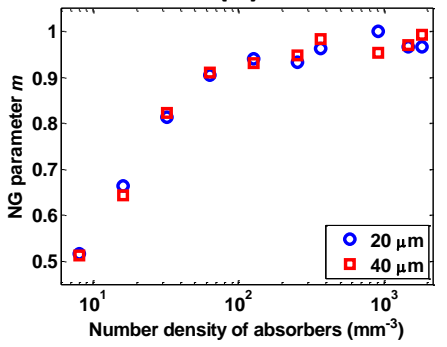

$10 \mathrm{~b} / \mathrm{rv}$
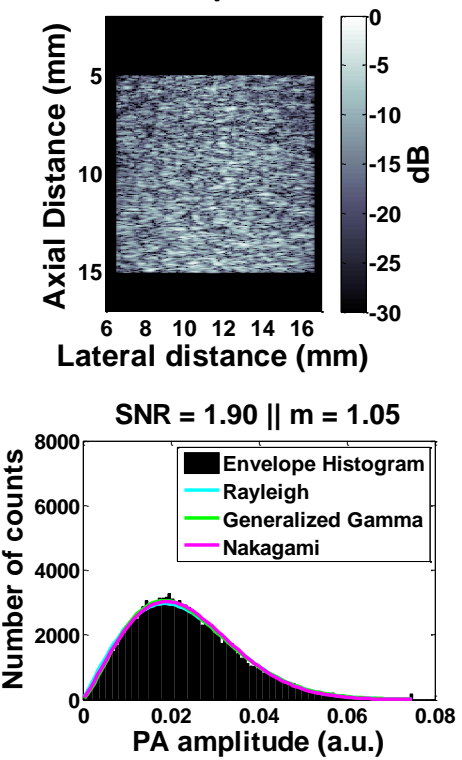

(e)

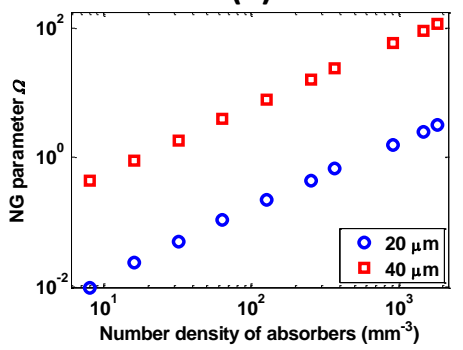

Figure 2-3: Simulation results for the envelope statistics method. (a) PA Bmode images of $5 \mu \mathrm{m}$ spherical bead phantoms for increasing b/rv. (b) Corresponding PA signal envelope histograms fitted to the Rayleigh, Generalized Gamma (GG) and Nakagami (NG) statistical distributions. (c) GG $a$, (d) NG $m$ and (e) NG $\Omega$ parameters plotted as a function of size and concentration.

\subsubsection{Radiofrequency spectroscopy}

Given that PA signal amplitude is affected by absorber morphology, size, concentration, optical absorption and laser fluence [160], it can be difficult to isolate the impact that any one of these factors has when imaging biological tissue [184]. Here, we investigate a subset of these parameters (size and concentration) in a well-controlled simulation. Figure 2-4a and Figure 2-4b show PA speckle in simulated PA B-mode images for various absorber sizes and degree of polydispersity. The mean image intensity increased with size $(14.5 \mathrm{x}$ from 10 to $30 \mu \mathrm{m})$ and polydispersity $(2 \mathrm{x}$ from 0.2 to $12.6 \mu \mathrm{m}$ ). Figure $2-4 \mathrm{c}$ shows the PA spectral slope (SS) as a function of bead radius for two different absorber concentrations. Increasing absorber size decreases the SS regardless of concentration, suggesting that the PA RF spectra contain information about the absorber size. This 


\section{CHAPTER 2}

is consistent with previous studies in which the SS was used to quantify microstructure in biological tissue and for red blood cell aggregation in blood suspensions [145], [185]-[187].

(a)
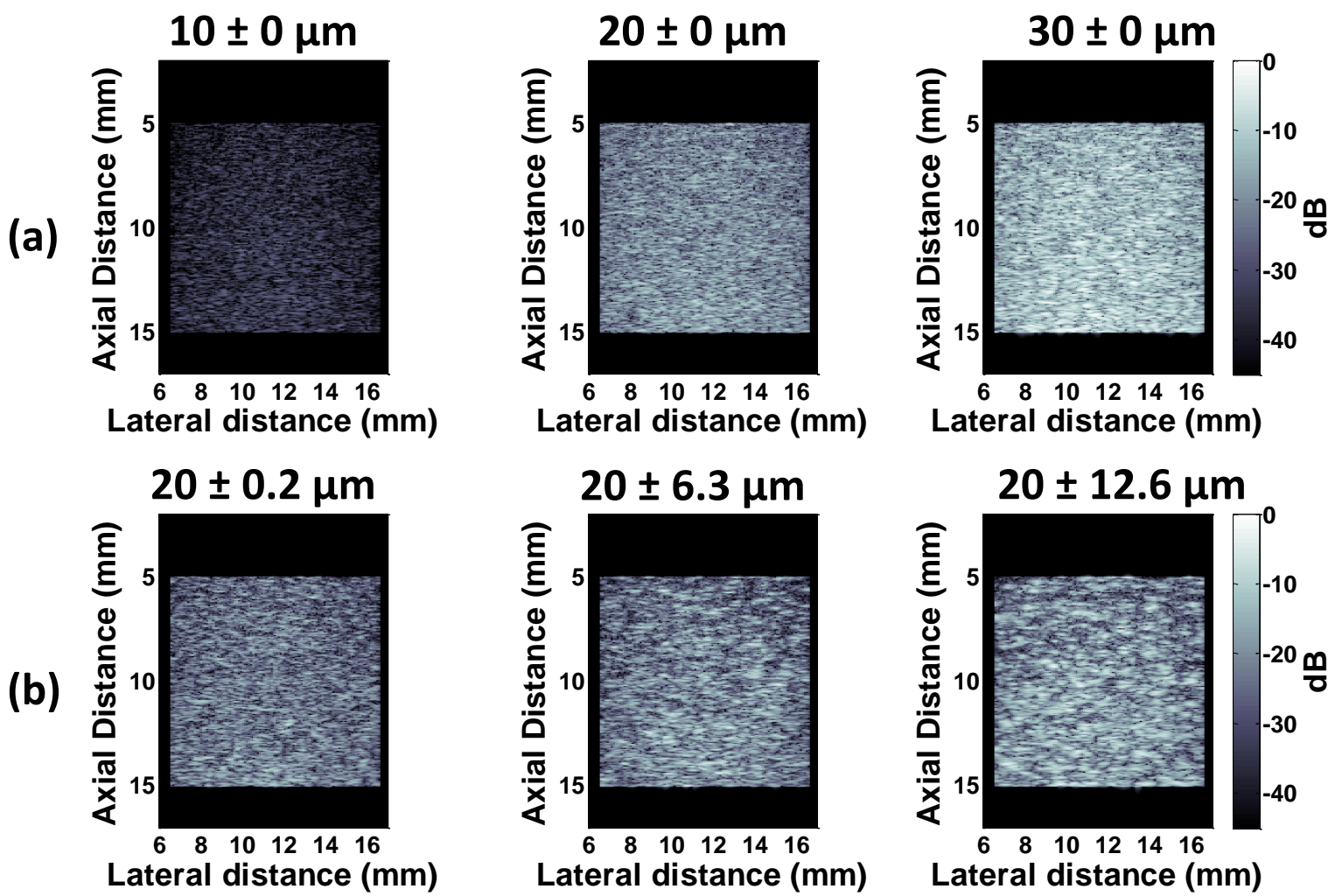

(c)

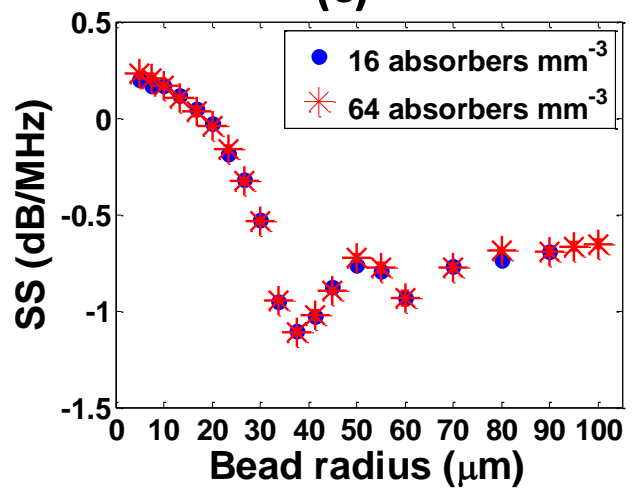

(d)

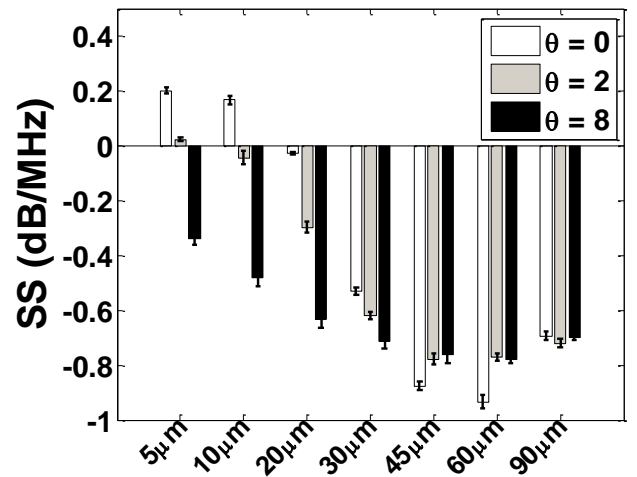

Figure 2-4: Simulation results for the radiofrequency spectroscopy method. PA B-mode images for (a) monodisperse and (b) polydisperse collection of beads at a concentration of 10 beads per resolution volume. SS as a function of (c) bead radius and concentrations and (d) polydispersity (controlled by the $\theta$ parameter).

These results suggest that the PA SS can be used to monitor changes in size without resolving absorbing structures. This is in agreement with previous PA studies at lower ultrasonic frequencies [188]. Figure $2-4 \mathrm{c}$ reveals that beyond $30 \mu \mathrm{m}$, the $\mathrm{SS}$ remains constant with increasing size for the 


\section{CHAPTER 2}

frequencies considered in these simulations. Thus, it may be possible to determine when the absorber radius is above or below a certain threshold (30 $\mu \mathrm{m}$ in this case) by computing the SS parameter from an experimentally acquired PA B-mode image. The SS curve flattening for larger sphere size seen in Figure $2-4 c$ is due to the relation of absorber size to the wavelength of the detected US wave. In US imaging, Rayleigh and Faran scattering regimes are defined for scatterering structures whose size is smaller and similar to/larger than the US wavelength, respectively [140]. Due to the broadband nature of PA signals, frequencies from equivalent regimes are present in PA imaging. For $k a>1$ (where $2 \pi f / c$ is the ultrasonic wavenumber of the detected ultrasound as determined by the transducer characteristics), the SS does not

significantly change with absorber size and no variations in spectral features (minima and maxima) are apparent. This is seen in Figure 2-4d where the PA SS becomes more negative for increasing polydispersity, but only up to $30 \mu \mathrm{m}$. In biological tissues, polydispersity exists at multiple biological length scales [95] but is difficult to identify in PA B-mode images alone (Figure 2-4b). The PA SS provides a tool for assessing the presence of polydispersity (observing a decreasing SS as a function of size) and quantifying its degree (changing magnitude of slope) with potential applications in monitoring vascular therapies longitudinally [133], [150]-[153].

\subsubsection{Cepstral analysis}

Figure 2-5a shows the geometries of numerical phantoms with various percentages of regularly spaced $10 \mu \mathrm{m}$ absorbers (denoted by the grid-to-random or G:R ratio). The number density of absorbers was $8 / \mathrm{mm}^{3}$. B-mode images are shown in Figure 2-5b. When all the absorbers are arranged in a grid pattern (i.e. $\mathrm{G}: \mathrm{R}=100: 0 \%$ ), their periodicity enhances the PA signal at specific spatial positions of the absorber (or, equivalently, absorbers separated by specific distances). It should be noted that the apparent size of the beads in Figure 2-5b is not an accurate representation of their physical dimensions $(10 \mu \mathrm{m})$ but determined by the transducer's point spread function.

As the percentage of randomly positioned absorbers increases, the PA image begins to look like the B-modes shown in Figure 2-3 and Figure 2-4 but fully developed speckle does not form (SNR $<$ 1.91). A challenge with such images is identifying whether the underlying tissue structure contains periodicity in the spatial arrangement of absorbers. This is of particular interest in imaging the vascular development in tumors [65]. The tumor vasculature does not have the structural 


\section{CHAPTER 2}

organization characteristic of normal tissue. PA imaging of whole tumors could in principle be used to quantify such changes in organization in cases where individual vessels cannot be fully resolved.

(a)
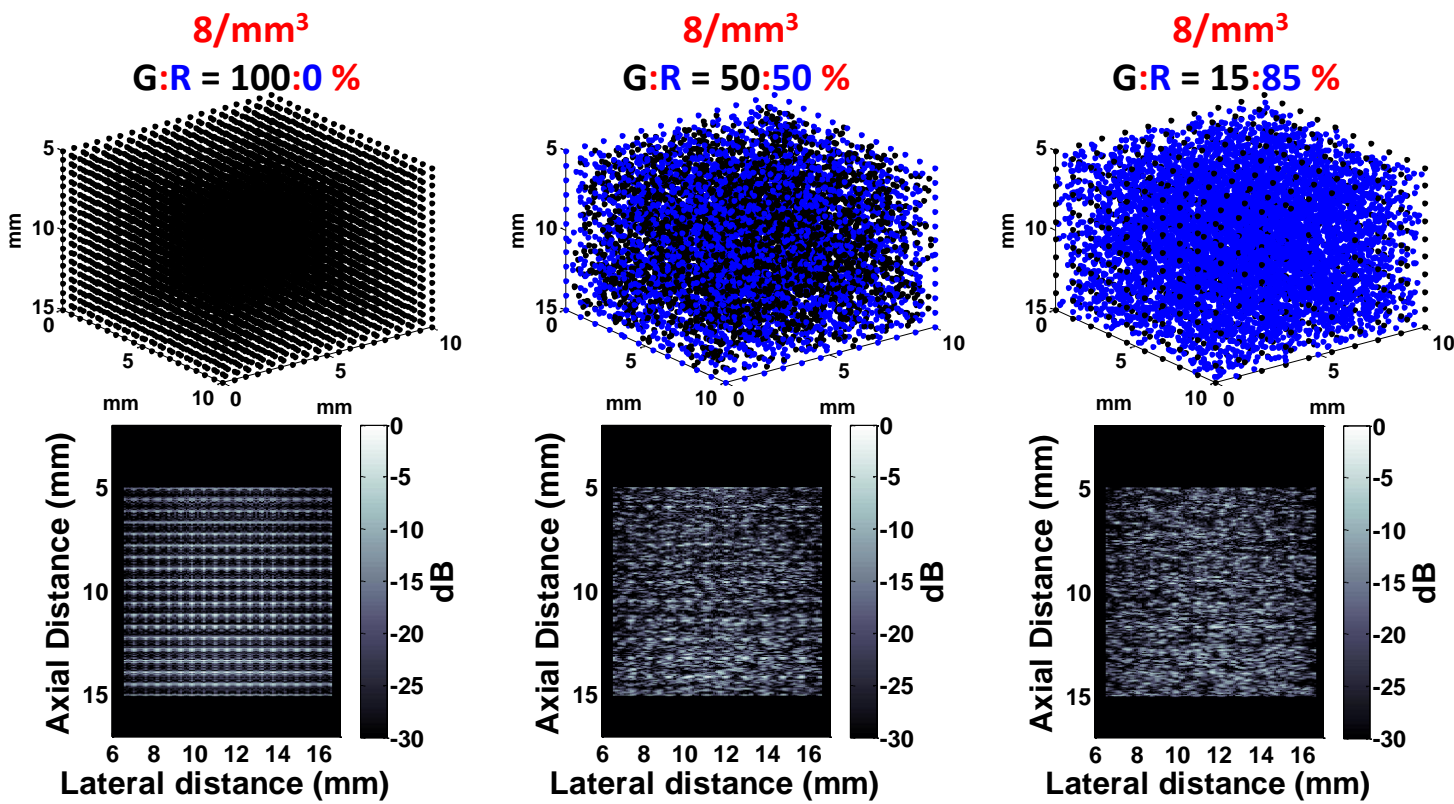

(c)
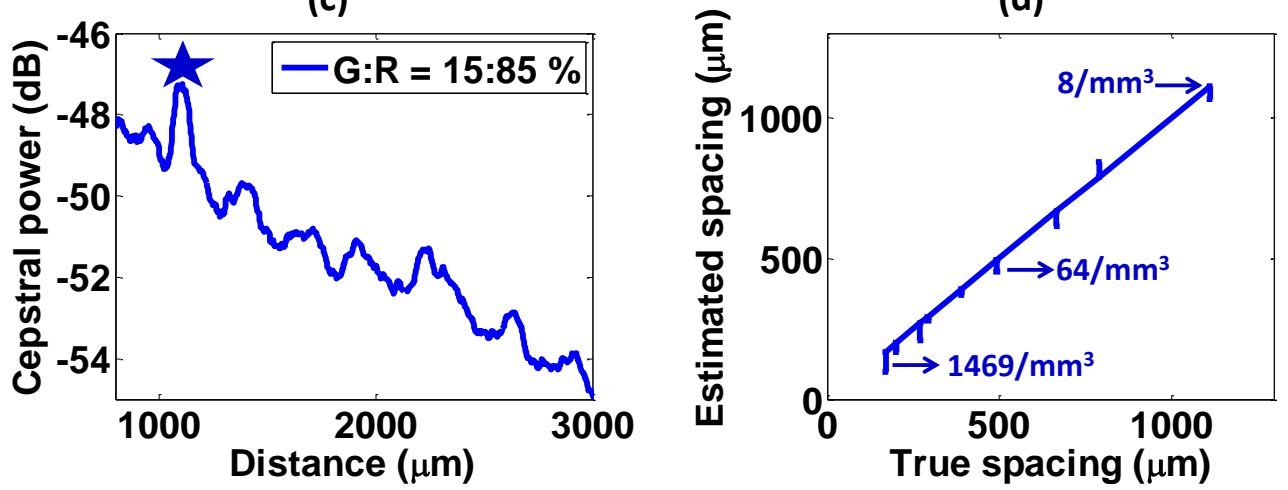

Figure 2-5: Simulation results using the cepstral analysis method. (a) Geometries of phantoms for various grid $(\mathrm{G})$ to random $(\mathrm{R})$ ratio and $(\mathrm{b})$ their corresponding PA B-mode images. (c) Power cepstrum of the 15:85\% phantom with star denoting the location of the first peak. (d) Comparison of the estimated absorber with the true spacing for various number densities.

Figure $2-5 \mathrm{c}$ shows the average power spectrum when $15 \%$ of all absorbers are arranged periodically. The location of the first cepstral peak (denoted by the star) is a measure of the most commonly occurring spacing between absorbers [174]. In the 15:85\% case, the periodic absorbers were spaced approximately $1 \mathrm{~mm}$ apart, with randomly positioned absorbers occupying the space between. The spacing calculated through spectral analysis in Figure 2-5c agrees well with the a priori known grid spacing. The cepstral-estimated absorber spacing was compared for various 


\section{CHAPTER 2}

absorber concentrations ranging from $8 / \mathrm{mm}^{3}$ to $1469 / \mathrm{mm}^{3}$ (fully developed speckle). The results, shown in Figure 2-5d, suggest that the technique can be used to estimate the most predominant spacing of an ensemble of sub-resolution absorbers (without having to resolve individual constituents). This demonstrates the potential of speckle cepstral analysis in biomedical applications such as monitoring therapeutic microwave lesions in the liver [189]. We believe that this approach can find applications in PA imaging using limited-view geometries for understanding the physical properties of various biological tissues such cancerous tumors [152] and liver [144].

\subsubsection{Experimental evidence for PA speckle}

Figure 2-6 shows experimental results of PA speckle formation in imaging systems of multiple transducer frequencies (5-400 MHz). Speckle is ubiquitous to all images and extends from the centimeter (Ultrasonix RP Figure 2-6a) to the micrometer (SASAM, Figure 2-6d) length scales. For the Ultrasonix RP (Figure 2-6a), the large-scale pattern with speckle forms in accordance to the shape of the diffuse light distribution profile (see Supplementary Figure 2.A(a)). Additionally, because of the limited-view geometry, speckle size changes as a function of depth. These effects are also present in the VevoLAZR system (Figure 2-6b) with light emission from two rectangular strips oriented at $30^{\circ}$ relative to the acoustic aperture [175]. SASAM images at 200 and $400 \mathrm{MHz}$ (Figure 2-6c and Figure 2-6d, respectively) reveal different speckle patterns for the same phantom. The speckle size at $400 \mathrm{MHz}$ is smaller than the $200 \mathrm{MHz}$ image, consistent with the effects of transducer properties on image speckle. 


\section{CHAPTER 2}

(a)

(b)

(c)
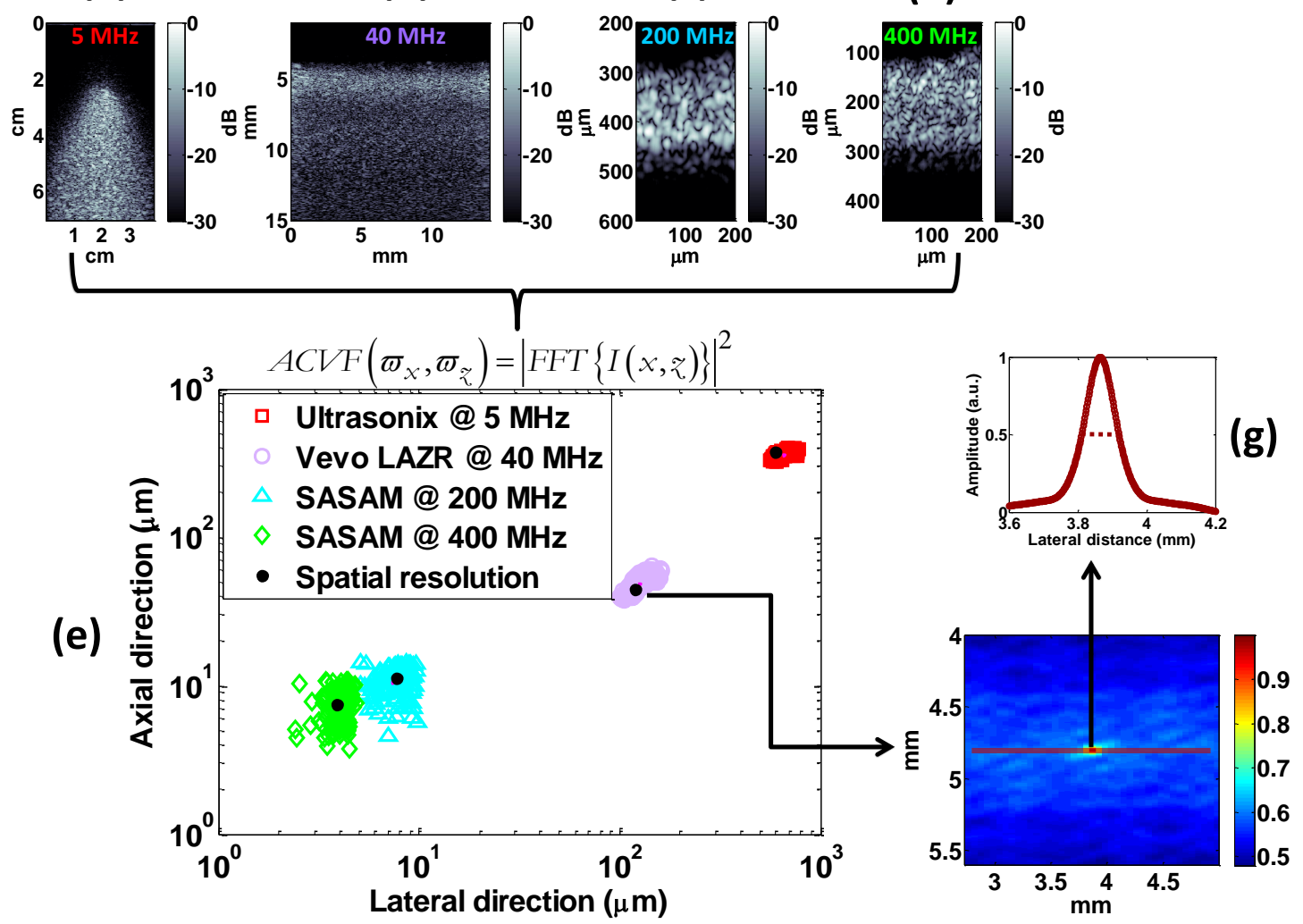

(f)

Figure 2-6: Effects of the imaging transducer on PA speckle size. PA B-mode images from speckle phantoms imaged with the (a) Ultrasonix RP, (b) VevoLAZR and the SASAM at (c) 200 and (d) $400 \mathrm{MHz}$. (e) Comparison between estimated speckle size and the spatial resolution of each system. (f) A representative 2D ACVF function from the VevoLAZR. (g) The lateral cross-section of the ACVF was used to estimate speckle size by measuring its FWHM.

Figure 2-6e shows the speckle size estimations for all transducers. The speckle size is obtained from the 2D ACVF (Figure 2-6f) along with the lateral profile of the ACVF maximum amplitude (Figure 2-6g) shown for the $40 \mathrm{MHz}$ probe. For each transducer, the speckle size was estimated from the FWHM of the horizontal and vertical line profiles through the center of the ACVF. The speckle size estimates for all systems were within $8.5 \%$ of the spatial resolution (see Supplementary Figure 2.C). The speckle size in US imaging predominantly depends on the physical properties of the imaging transducer [173]. The transducer pulse bandwidth and beam width significantly affect the qualitative appearance of US speckle texture. As the size of the transducer focal zone decreased, the lateral width of the speckle, similar to US imaging. This was observed in images of the same phantom with two different transducers (Figure 2-6c and Figure 2-6d). Moreover, the calculated ACVF is comparable to the resolution volume [158], with axial 


\section{CHAPTER 2}

speckle size inversely proportional to pulse bandwidth and the lateral speckle size increasing with range and beam width.

Figure 2-7 shows the application of the three analysis techniques (outlined in Figure 2-2) to PA phantom images. The statistical distributions of the signal envelope histograms (Figure 2-7a to Figure 2-7d) establish that fully developed speckle formed across all the imaging systems spanning frequencies of 5 to $400 \mathrm{MHz}$. The criteria for fully developed speckle [172], namely the Rayleigh SNR equal to 1.91 and the Nakagami $m$ parameter approaching 1, were met for all phantoms. This agrees with the simulation results (Figure 2-3). When comparing two different sizes of absorbers (e.g. 3.5 and $15 \mu \mathrm{m}$ ), the Generalized Gamma $a$ fit parameter to the envelope histograms (Figure $2-7 \mathrm{e}$ ) increases by $4.5 \mathrm{x}$ with increasing size. The PA SS of the normalized spectra decreases by a factor of $1.4 \mathrm{x}$ with increasing absorber size. The decrease in SS is consistent with the simulation results shown in Figure 2-4. The power cepstra (Figure 2-7f) reveal the presence of a peak for the $15 \mu \mathrm{m}$ phantom around $220 \mu \mathrm{m}$ while no discernable peak is visible for the phantom containing 3 $\mu \mathrm{m}$ beads. Even though these phantoms contain a randomized distribution of absorbers, it might be possible that periodicities might arise within the phantom for higher concentration of absorbers. The location of the cepstral peak would depend on spacing of the absorbers within one resolution volume [174]. Moreover, the prominence/amplitude of the peak would be affected by the degree of randomness in the distribution of absorbers. Comparing these peaks to the simulation results shown in Figure 2-5c, one might postulate that the number density of periodically spaced absorber is most likely smaller than $15 \%$ in the entire phantom. 


\section{CHAPTER 2}

(a)

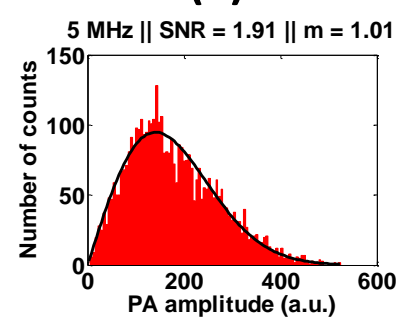

(b)

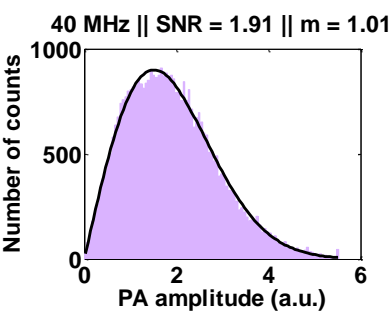

(c)

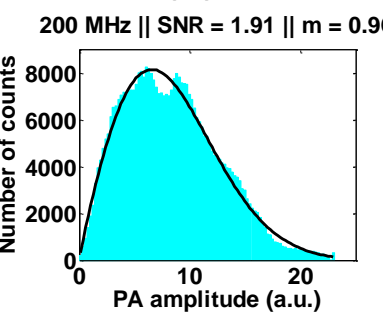

(d)

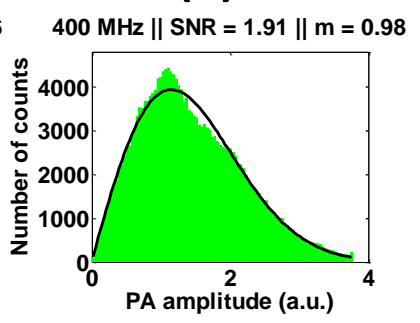

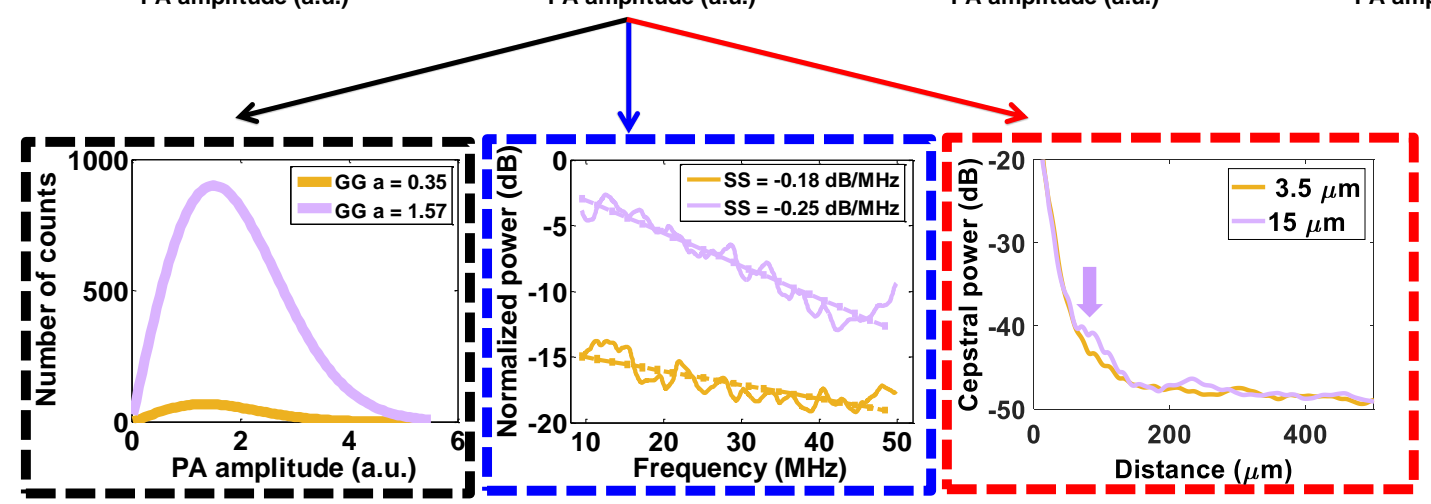

\section{Envelope statistics}

(e)
Radiofrequency spectroscopy
Cepstral analysis

(g)

\section{(f)}

Figure 2-7: Speckle analysis techniques applied to experimental phantoms. Nagakami fits to the PA signal envelope for the phantoms imaged at (a) $5 \mathrm{MHz}$, (b) $40 \mathrm{MHz}$, (c) $200 \mathrm{MHz}$ and (d) 400 MHz. The calculated SNR and Generalized Gamma $m$ parameter are provided on top of each figure. For the VevoLAZR $40 \mathrm{MHz}$ probe, the three analysis techniques presented in this paper, namely (e) Envelope statistics, (f) Radiofrequency spectroscopy and (g) Cepstral analysis were applied to phantoms consisting of 3 and $15 \mu \mathrm{m}$ polystyrene beads. The arrow denotes a peak forming for the $15 \mu \mathrm{m}$ absorber phantom.

\subsubsection{Photoacoustic speckle from tumor vasculature}

\subsubsection{B-mode images from simulated and measured tumor blood vessels}

Tumor angiogenesis is required to sustain the metabolic demands of the tumor growth [64]. The tumor vasculature typically does not have the hierarchical organization present in normal tissue [177]. Fractal models of the vasculature offer a means of studying vascular growth by focusing on the size, shape and orientation of each vessel while modeling the PA wave propagation from vascular trees. The effect of vessel size and number was studied using models of vascular architecture for breast tumors [150], [151]. Figure 2-8a shows the simulation geometry and PA Bmode images of simulated vascular trees at 7- and 14-days post-inoculation. Using histologically 


\section{CHAPTER 2}

measured data of vessel diameters for breast tumors in mice [151], we simulated a representative subset of the vascular architecture. The simulated PA B-mode images of the vascular trees show the presence of speckle arising from several hundred non-resolvable vessels at various orientations contained within the imaging resolution volume. Much like the spherical absorbers shown in Figure 2-3 - Figure 2-6, cylindrical PA sources also give rise to speckle when a sufficient number are contained within the resolution volume of the imaging transducer. Speckle is also present in the US and PA images of the in-vivo mouse tumor (Figure 2-8b). It is present at all stages of mouse tumor growth vessel growth. Changes in the vasculature with tumor growth were modeled by increasing the branching order (and therefore the total number of vessels), consistent with previous in-vivo reports [180], [181].

The US and PA speckle patterns are similar to each another for the in-vivo mouse tumors (Figure 2-8b). Supplementary Figure 2.D shows that estimates of the speckle size for US and PA images

of tumors are within $10 \%$ of the spatial resolution of the VevoLAZR transducer used to image these tumors. However, the speckle texture differs between PA simulations and experiments. While the experimental tumor speckle size is comparable to the transducer spatial resolution, the lateral size of the simulated speckle (estimated from Eq. (2.8)) is larger than the lateral resolution. This could be because the directivity of the linear array implemented in the simulations through Eq. (2.3) might differ from the true directivity of the transducer. Additionally, the effects of light fluence [190] and acoustic attenuation [191] on PA images are not taken into account, potentially affecting the pattern observed.

\subsubsection{Radiofrequency spectroscopy analysis from tumor vasculature}

Figure 2-8c - Figure 2-8f show analysis of the growing tumor vasculature using radiofrequency spectroscopy and cepstral analysis. In simulations and in-vivo tumors, the PA SS (Figure 2-8c and Figure 2-8e) decreases by $20 \%$ and $17 \%$, respectively as the tumor vasculature network grows in size from 7 to 14 days. The decrease in SS suggests that the average absorber size increases as the tumor grows [180]. Our previous work with vascular targeted treatments [151] has shown that the PA SS can be used to monitor changes in vessel size within tumors post vascular disrupting treatment. These results suggest that PA radiofrequency spectroscopy has the potential to differentiate changes in tumor vasculature (either due to tumor growth or treatment). This is an 


\section{CHAPTER 2}

area of interest to multiple research areas, including drug discovery [192], where modalities such as PA imaging can have a translational impact.

(a)

Numerical simulations of tumors
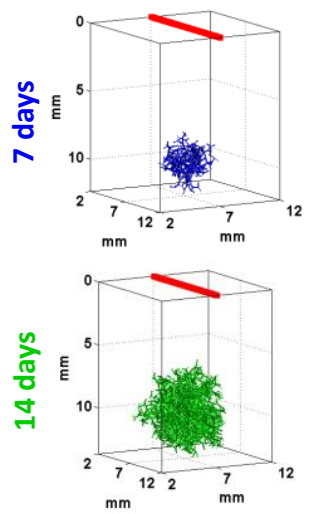

(c)

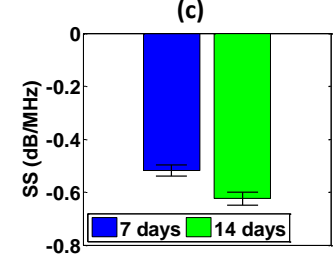

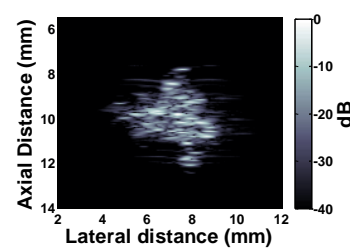

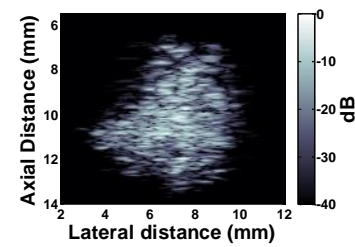

(d)

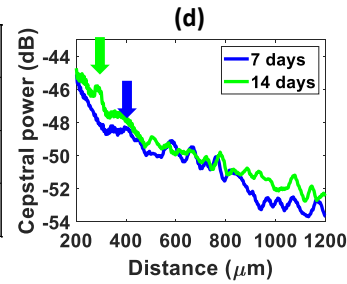

(b)

\section{In-vivo measurements of tumors}
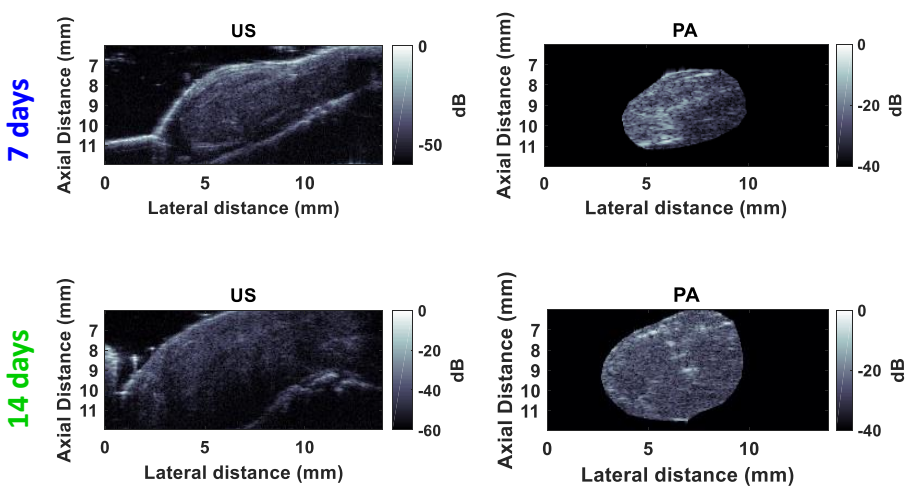

(e)
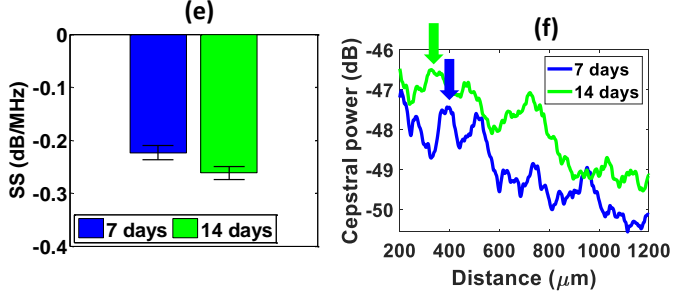

Figure 2-8: PA speckle from simulated and experimentally measured tumor vasculature. (a) Geometry of the vascular tree alongside the PA B-mode for the numerical simulations of breast tumor vasculature. The average vessel diameters used for the simulated vasculature were $37.8 \mu \mathrm{m}$ and $48.2 \mu \mathrm{m}$ at 7 and 14 days, respectively, and were all based on histological sectioning of invivo tumors. (b) US and PA images $(750 \mathrm{~nm})$ of in-vivo EMT-6 tumors at $7(\mathrm{n}=3)$ and $14(\mathrm{n}=4)$ days post-inoculation. (c)+(e) Spectral slope and (d)+(f) power cepstra for the numerical simulations of tumors modeling vascular growth and the in-vivo EMT-6 tumors. The arrows denote the location of the largest amplitude cepstral peaks.

\subsubsection{Cepstral analysis from tumor vasculature}

Cepstral techniques can be used to probe the spatial arrangement of tumor blood vessels by identifying periodicities arising from non-resolvable absorbing structures. Figure 2-8d and Figure 2-8f show the average cepstra for simulated mouse xenograft breast tumor vasculature networks as well as in-vivo tumors. Both simulation and experiment contain an increasing number of total vessels from 7 to 14 days post-inoculation. As the overall number of vessels increased, the location of the most prominent (largest amplitude) peak in the cepstra decreased by $100 \mu \mathrm{m}$ for both simulations (Figure 2-8d) and experiments (Figure 2-8f). The presence of ceptral peaks suggests the existence of periodicities in the vessel spatial distribution. These periodicities can arise due to 


\section{CHAPTER 2}

specific vessel separation distances in the chaotic tumor vasculature being more common than others. Increasing the number of blood vessels in simulations or allowing the tumors to grow to 14 days (in-vivo) reduces the physical separation between each vessel. This is expected to result in a decrease in the mode (i.e. most commonly occurring) of the blood vessel separation distance. While tumor vasculature is known to lose much of its periodicity and order [177], [179], it is possible that scant organization detectable with the cepstral analysis technique remains. While it may be the case that fewer than $10 \%$ of vessels have organized, periodic spacing, the results shown here point to differences between the periodic spacing of vessels in the 7 and 14-day old tumors.

According to the simulations and experiments, it might be possible to study the spatial separation of non-resolvable blood vessels in a tumor using cepstral analysis [174]. The PA signal detected is a superposition of PA waves, with greater contributions to the signal energy from vessels of the relevant length scale (as determined by the ultrasound wavelengths associated with the detection transducer bandwidth). The branching level of the vascular tree that would principally contribute to the PA signal is determined by a combination of the PA signal strength of the vessel size at that branching level and the detection bandwidth of the US transducer [193]. The former is itself a combination of the size and number of such vessels within the transducer resolution volume. Cepstral peaks could be associated with spacings at that branching level. Future studies will focus on understanding the location of cepstral peaks and how these are related to the structural organization of vessels within growing tumors [194].

\subsubsection{Remarks on the nature of speckle in photoacoustic imaging}

Our experimental findings show that PA speckle arises when PA waves from sub-resolution sources interfere. PA speckle is deterministic and can be described using speckle statistics. There are numerous references that describe PA imaging as a "speckle-free" modality [127], [161], [162]. The results of this work show that is not always the case. The presence of speckle in PA imaging depends on the imaging approach, as it is present in acoustic-resolution PA imaging but not in the optical resolution PA imaging. The pressure of the waves generated in PA imaging are more broadband compared to the backscattered waves in US imaging. The transducers and acquisition hardware act as lowpass or bandpass filters suppressing the high frequency components of the inherently broadband PA signals. In acoustic-resolution resolution PA imaging there are instances 


\section{CHAPTER 2}

in which speckle is suppressed compared to the signal from boundaries. For example, when imaging individual blood vessels with diameters larger than the acoustic resolution volume, the vessel boundaries are accentuated, and the comparatively weak signal from speckle in the vessel interior cannot be readily appreciated. These effects are especially prominent in the cases of images with limited dynamic range [127] and occur in US imaging as well. However, as we demonstrate here, the inability to visualize PA speckle in such cases does not preclude its existence.

Formation of PA speckle from the tumor vasculature using limited-view geometries demonstrates the fundamental nature of the concept in-vivo. Much like the spherical absorbers shown in Figure 2-1 through Figure 2-6, cylindrical PA sources also give rise to speckle when a sufficient number are located within a resolution volume of the imaging transducer. PA speckle texture is also affected by acoustic attenuation [191] and light fluence [190] which were not incorporated into the theoretical model. Additionally, in-vivo tumors contain an unknown, but rather significantly large number of capillary beds [64] that would increase the number of non-resolvable PA absorbers in the transducer resolution volume. Moreover, for some imaging resolution volumes, vessels of the order larger than the resolution volumes exist. These parameters would influence the appearance of the speckle texture. Accounting for these effects would allow for more direct comparisons to experimentally measured tumors using limited-view PA geometries [133], [136], [137], [150], [151], [195]. The presence of speckle in PA imaging may be perceived as undesirable noise, as it is sometimes in US imaging, where it can obscure boundaries. However, since it is a deterministic signal, it has potential applications in the tissue characterization and speckle tracking [196].

\subsection{Conclusions}

Speckle in PA imaging arises from the spatiotemporal superposition of non-resolvable absorbers. In this work, we demonstrate that speckle carries information about the underlying absorber structure of individual sub-resolution sources. This paper introduced several analysis techniques (envelope statistics, radiofrequency spectroscopy and cepstral analysis) that can be applied to acoustic-resolution PA imaging with speckle. A fractal-based vascular model of tumors revealed that PA speckle also arises from complex tumor geometries consisting of cylindrical sources. These models were corroborated using PA data acquired from mouse tumors in-vivo. We also demonstrated the feasibility of the analysis techniques in quantifying absorber size and 


\section{CHAPTER 2}

distribution. These findings have potential applications in monitoring changes in vessel size during vascular targeted cancer therapies.

\subsection{Acknowledgments}

This work was funded by the Natural Sciences and Engineering Research Council of Canada (NSERC), the Terry Fox Foundation and the Canada Research Chairs Program. EH is supported by an NSERC Vanier Canada Graduate Scholarship. The authors would like to acknowledge Drs. Ping Gong, Sankar Narasimhan, Ratan Saha and Subhajit Karmakar for their insights in the theoretical discussions of speckle. The assistance of Drs. Jonathan May, Lauren Wirtzfeld and Shyh-Dar Li and Elijus Undzys for the in-vivo experiments is gratefully acknowledged. 


\subsection{Supplementary information}

\subsubsection{Supplementary Figure 2.A}

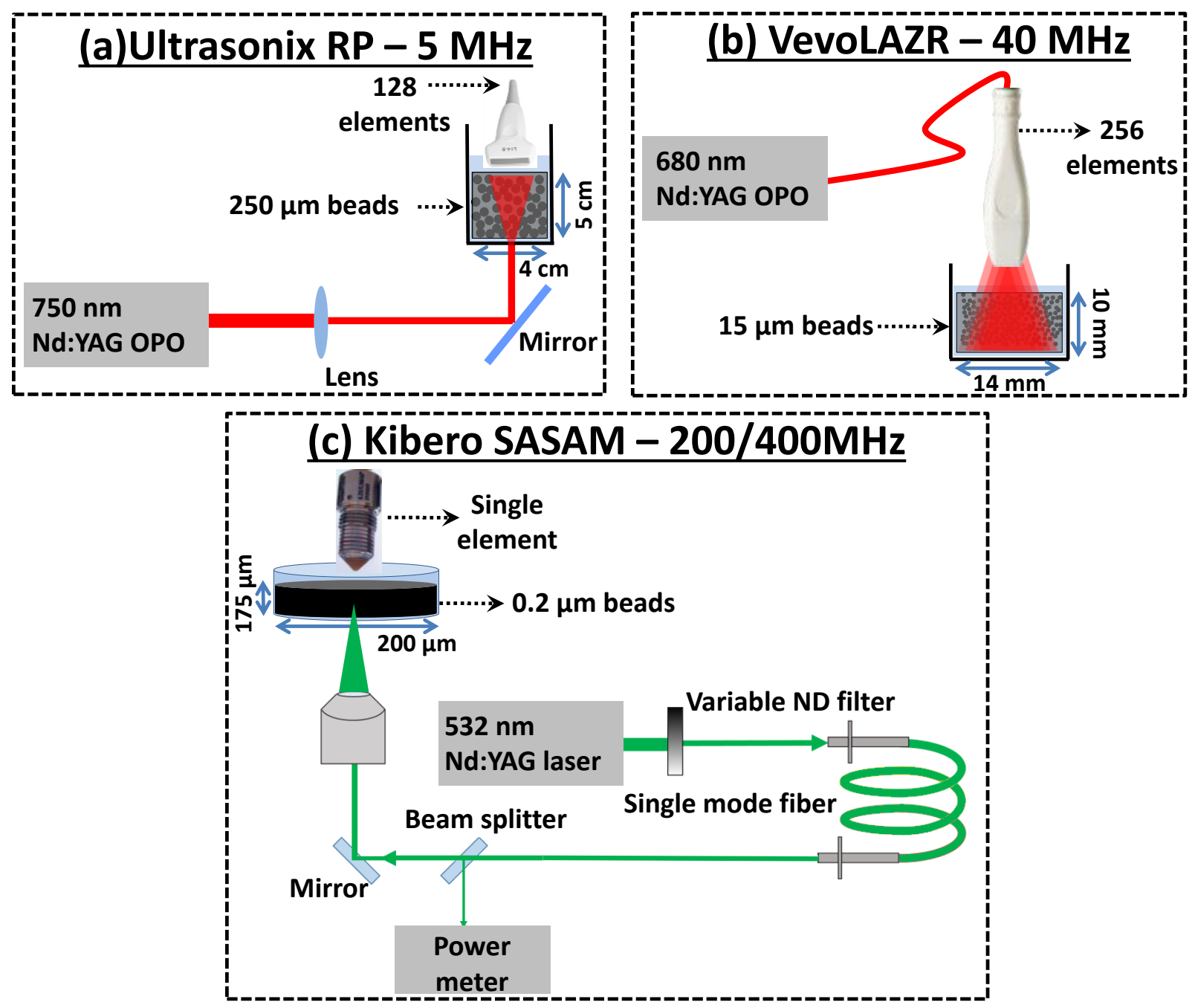

PA imaging setups for the (a) Ultrasonix RP (5 MHz linear array, 128 elements) (b) VevoLAZR (40 MHz linear array, 256 elements). (c) Kibero SASAM (200 MHz and $400 \mathrm{MHz}$ single element transducers).

(a) Ultrasonix RP clinical system (BK Ultrasound, Richmond, BC, Canada)

The laser source was an Nd:YAG-pumped optical parametric oscillator (OPO, Opotek Inc., Carlsband, CA, USA) operating at $760 \mathrm{~nm}$. It was focused into the phantom using a focusing lens and a mirror tilted at $45^{\circ}$. The laser was operated at a $10 \mathrm{~Hz}$ pulse repetition frequency (PRF), with each pulse emission triggering the acquisition of a passive $5 \mathrm{MHz}$ center frequency linear array 


\section{CHAPTER 2}

transducer with $7 \mathrm{MHz}-6 \mathrm{~dB}$ bandwidth. The non-beamformed PA signals were acquired using an external DAQ attached to the RP unit. A total of 15 frames were acquired and beamformed offline using a delay-and-sum approach prior to temporal averaging of all frames at $14 \mathrm{~Hz}$ frame rate.

\section{(b) VevoLAZR pre-clinical system (Fujifilm-VisualSonics Inc., Toronto, ON, Canada)}

A linear array probe with 256 elements and $40 \mathrm{MHz}$ center frequency (41 MHz -6 dB bandwidth) with an integrated laser operating at $680 \mathrm{~nm}$ was used to image the phantom through water. The laser source was a Nd:YAG pumped OPO (Opotek Inc.) operating at $10 \mathrm{~Hz}$ PRF [175]. The light is emitted from two rectangular strips on both sides of the acoustic aperture of the transducer, at a $30^{\circ}$ angle relative to the imaging plane. A total of 5 non-beamformed PA frames were acquired, beamformed and averaged offline.

\section{(c) Kibero SASAM photoacoustic microscope (Kibero GmbH, Saarbrucken, Germany)}

The SAarland Scanning Acoustic Microscope (SASAM) was used with two different single element focused transducers operating at center frequencies of $200 \mathrm{MHz}$ (aperture diameter/focal depth $500 \mu \mathrm{m}, 138 \mathrm{MHz}-6 \mathrm{~dB}$ bandwidth) and $400 \mathrm{MHz}$ (aperture diameter/focal depth $350 \mu \mathrm{m}$, $218 \mathrm{MHz}-6 \mathrm{~dB}$ bandwidth). A fiber-coupled $532 \mathrm{~nm} \mathrm{Nd:YAG} \mathrm{laser} \mathrm{(Teem} \mathrm{Photonics,} \mathrm{Meylan,}$ France) operating at $4 \mathrm{kHz}$ PRF was used to generate the PA waves within the phantom. A variable neutral density (ND) filter (Thorlabs Inc., Newton, NJ, USA) was used to vary the energy at the input end of a single mode fiber (Costal Connections, Venura, CA, USA). The laser output was passed through a beam splitter; the transmitted portion was focused onto the phantom using a $4 \mathrm{X}$ microscope objective, and the reflected portion was directed into a Mach6 energy meter (Gentec Electro-Optics Inc., Quebec City, QC, Canada). The transducer and laser were coaxially aligned and a phantom region of $200 \mu \mathrm{m} \times 100 \mu \mathrm{m}$ was scanned through the confocal zone. A total of five B-mode images were acquired $20 \mu \mathrm{m}$ apart and were spatially averaged. The same phantom was imaged using both the 200 and $400 \mathrm{MHz}$ transducers. 


\section{CHAPTER 2}

\subsubsection{Supplementary Figure 2.B}

(a)

\begin{tabular}{||c|c|c|c||}
\hline $\begin{array}{c}\text { Imaging system } \\
\text { Transducer frequency }\end{array}$ & $\begin{array}{c}\text { Transducer design } \\
\text { Laser characteristics }\end{array}$ & $\begin{array}{c}\text { Bead } \\
\text { diameter }\end{array}$ & $\begin{array}{c}\text { Imaging field } \\
\text { of view }\end{array}$ \\
\hline \hline Ultrasonix $/ / 5 \mathrm{MHz}$ & 128 element array//750 nm OPO & $250 \mu \mathrm{m}$ & $5 \times 4 \mathrm{~cm}$ \\
VevoLAZR//40 MHz & 256 element array//680 nm OPO & $15 \mu \mathrm{m}$ & $10 \times 14 \mathrm{~mm}$ \\
$\mathrm{SASAM} / / 200 \mathrm{MHz}$ & 1 element, f\# $1 / / 532 \mathrm{~nm} \mathrm{Nd:YAG}$ & $200 \mathrm{~nm}$ & $175 \times 200 \mu \mathrm{m}$ \\
$\mathrm{SASAM} / / 400 \mathrm{MHz}$ & 1 element, f\#1//532 nm Nd:YAG & $200 \mathrm{~nm}$ & $175 \times 200 \mu \mathrm{m}$ \\
\hline
\end{tabular}

(b)

(c)
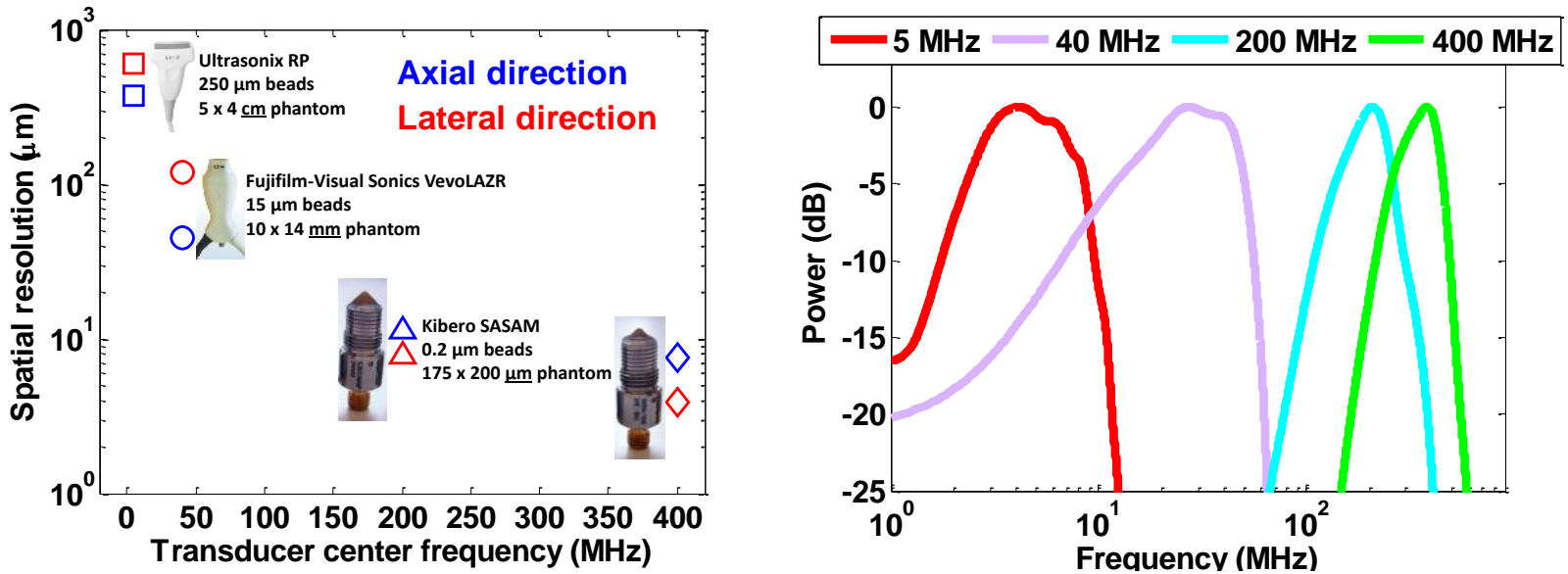

(a) Summary of the characteristics of the phantoms and the PA imaging system. (b) Spatial resolution of each transducer as a function of its center frequency. (c) PA frequency response for the transducers using in this study measured using a $200 \mathrm{~nm}$ gold film.

\section{Phantom preparation}

Phantoms imaged by the linear arrays were prepared using $10 \%(\mathrm{w} / \mathrm{v})$ porcine skin gelatin while the phantom imaged by the single element system consisted of 1.5\% (w/v) agarose (Sigma-Aldrich Co., St. Louis, MO, USA) as the suspending medium. The concentration of gelatin was chosen in order to achieve a frequency dependent attenuation of approximately $0.25 \mathrm{~dB} \mathrm{~cm}^{-1} \mathrm{MHz}^{-1}$. The optical absorbers for each system were: (i) $5 \mathrm{MHz}$ linear array: $250 \mu \mathrm{m}$ black glass beads (Corpuscular Inc., Cold Spring, NY, USA); (ii) $40 \mathrm{MHz}$ linear array: $15 \mu \mathrm{m}$ black polystyrene beads (Merck Millipore, Pithiviers, France); (iii) 200/400 MHz single elements: $0.2 \mu \mathrm{m}$ black polystyrene beads (Polysciences Inc., Warrington, PA, USA). The number of beads per each phantom was calculated based on the criterion that at least 10 beads per resolution volume of each imaging transducer.

In order to construct the phantoms imaged by the linear array systems, degassed water was heated to $35^{\circ} \mathrm{C}$ along with the beads before the gelatin powder was slowly added. This suspension was 


\section{CHAPTER 2}

magnetically stirred in order to achieve homogeneity until it reached $65^{\circ} \mathrm{C}$ and the gelatin was entirely dissolved. It was then placed inside a custom-made rotisserie for 3 hours at room temperature to ensure homogenous mixing of the beads inside each phantom. Each phantom was then kept at $4^{\circ} \mathrm{C}$ for 24 hours prior to imaging in order to achieve full solidification of the gelatin. The $0.2 \mu \mathrm{m}$ beads for the single element transducer phantom were centrifuged, supernatant aspirated and then re-suspended in molten agarose. The agarose was then sandwiched between two microscope coverslips and kept at $4^{\circ} \mathrm{C}$ for 15 minutes.

\subsubsection{Supplementary Figure 2.C}
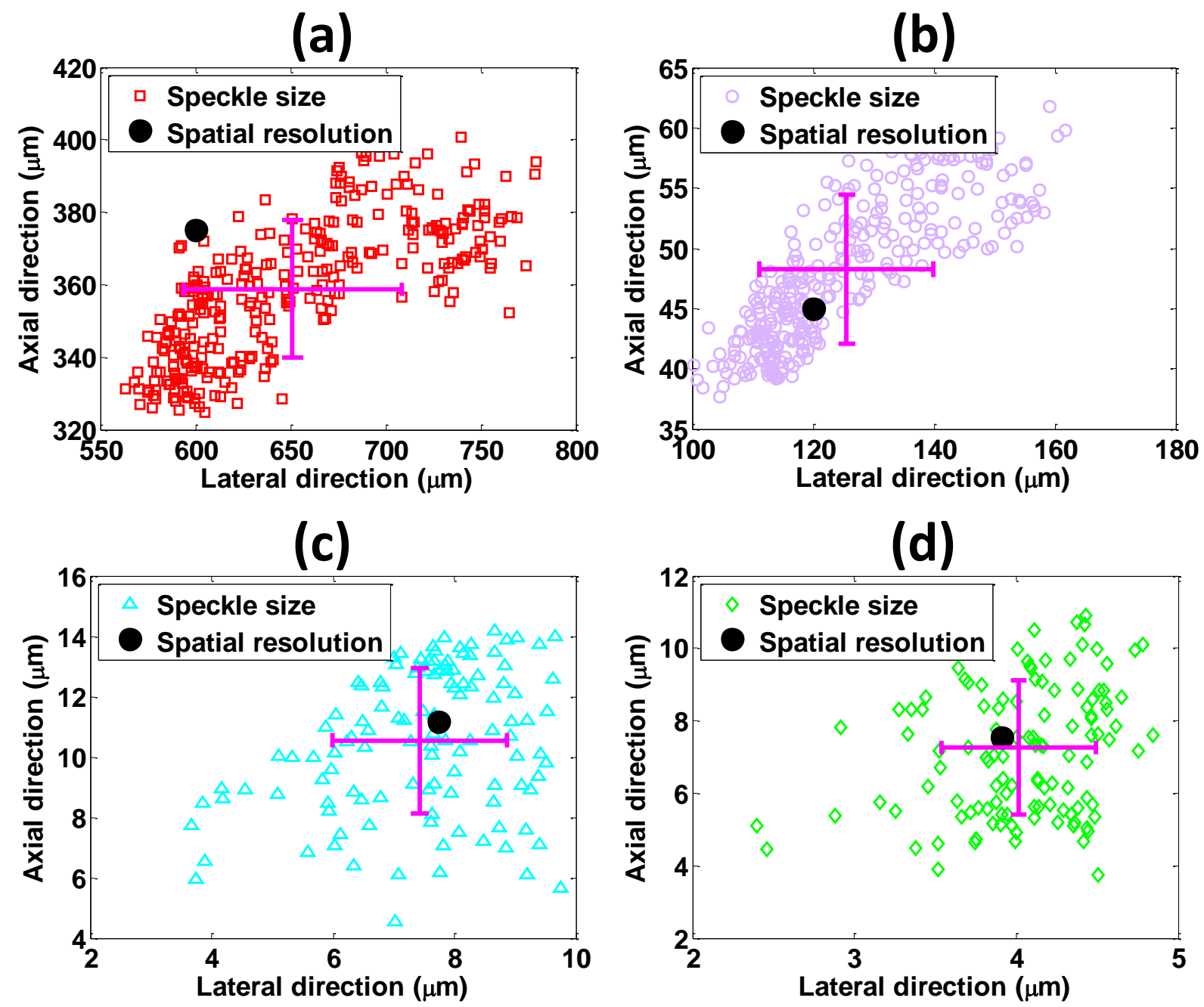

Speckle size estimations and comparison with spatial resolution for (a) the Ultrasonix RP, (b) the VevoLAZR and the Kibero SASAM at (c) $200 \mathrm{MHz}$ and (d) $400 \mathrm{MHz}$ in the axial and lateral directions. Each symbol represents the average speckle size estimated from a sliding window ROI through each image. Cross bars represent the standard deviation of the mean speckle size estimates in both dimensions. 


\section{CHAPTER 2}

\subsubsection{Supplementary Figure 2.D}
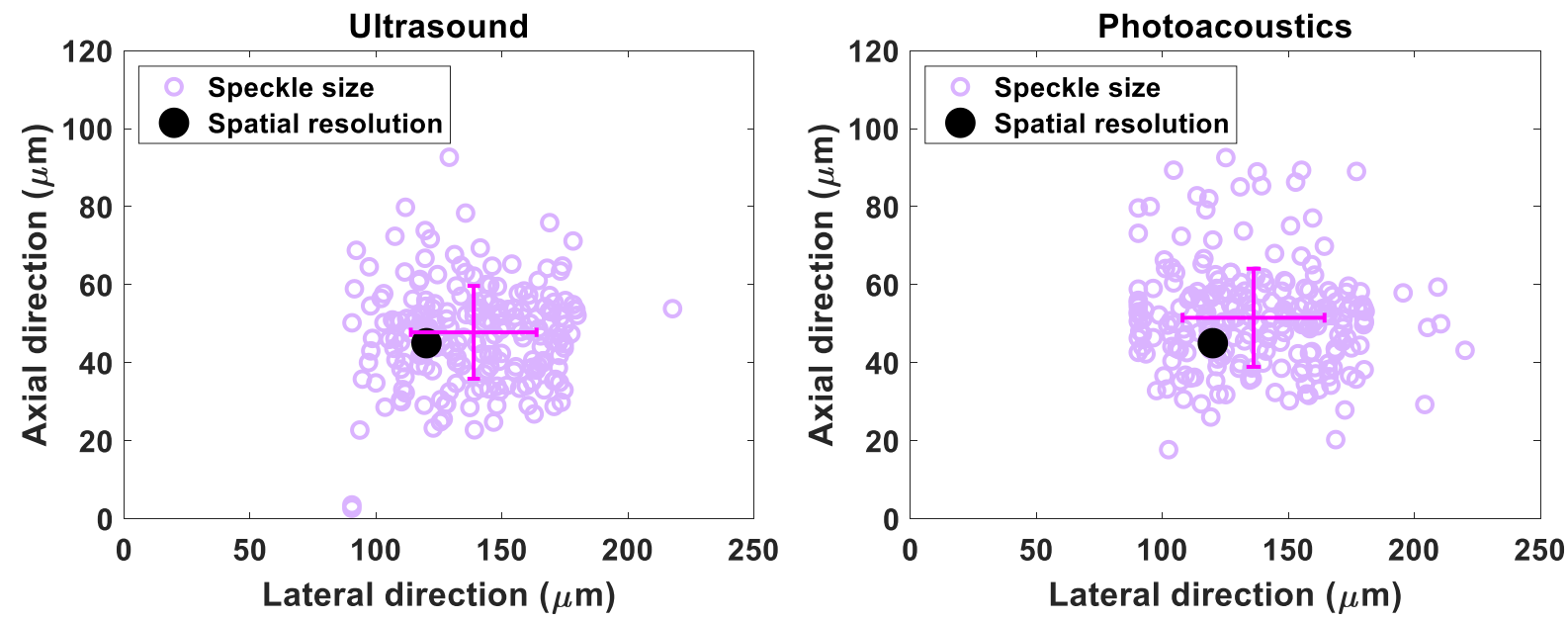

Ultrasound and photoacoustic speckle size estimates for a representative Balb/c mouse EMT-6 tumor imaged 14 days post-inoculation. The images were acquired with the VevoLAZR system at $40 \mathrm{MHz}$ and $750 \mathrm{~nm}$ illumination. Each circle represents the average speckle size estimated from a sliding window ROI through each image. Cross bars represent the standard deviation of the mean speckle size estimates in both dimensions. 


\section{Photoacoustic imaging of cancer treatment response: Early detection of therapeutic effect from thermosensitive liposomes}

The contents of this chapter have been previously published in PLOS ONE. Permission to reproduce this article was granted by the Public Library of Science and is provided in Appendix A.1.2.

JP May ${ }^{*}$, E Hysi ${ }^{*}$, LA Wirtzfeld, E Undzys, S-D Li and MC Kolios. "Photoacoustic imaging of cancer treatment response: Early detection of therapeutic effect from thermosensitive liposomes" PLOS ONE 2019; 11(10): e0165345 - https://doi.org/10.1371/journal.pone.0165345

*These authors contributed equally to this work.

\subsection{Introduction}

During cancer treatment it is normal practice to monitor the tumor for changes indicative of treatment response. Conventionally, studies monitor volumetric changes in tumor size which typically occur weeks after the administration of treatment and thus are not suitable as markers of early treatment response [197]. Instead, dynamic or functional imaging techniques capable of monitoring the relative effectiveness of drug delivery [198]-[201], or better still detecting the corresponding therapeutic effect, during or immediately after treatment are highly sought after [202]. With this in mind, we recognized that many drugs and delivery therapies induce changes to the tumor microenviroment long before the overall volume visibly changes, and through further investigation it might be possible to use these for an early detection method of therapeutic effect.

TSLs are a drug delivery technology that allows the targeting of a drug payload to a localized area through the application of mild-hyperthermia $\left(39-42^{\circ} \mathrm{C}\right)$ [203], [204]. The release temperature of a TSL can be tuned though the incorporation of lipids with different transition temperature $\left(\mathrm{T}_{\mathrm{m}}\right)$ or by adding other compounds (e.g. lyso-lipids, surfactants) to the lipid membrane. This approach has the potential to be particularly effective in cancer treatment, where heating (and so the drug release) can be confined to just the tumor area. This minimizes the uptake of drug elsewhere in the body and significantly reduces any unwanted side-effects associated with chemotherapy regimens [205]. A feature of the most clinically advanced ultra-fast temperature sensitive liposomes (uTSLs) is their ability to rapidly burst-release their drug payload (in seconds) when entering an area heated 


\section{CHAPTER 3}

to mild-hyperthermia, but remain intact and retain the majority of their payload (for more than an hour) at normal physiological temperatures (Figure 3-1). Hence, mild hyperthermia is usually applied within the first hour or two of uTSL treatment, for maximized concentration of encapsulated drug in circulation.

(a)

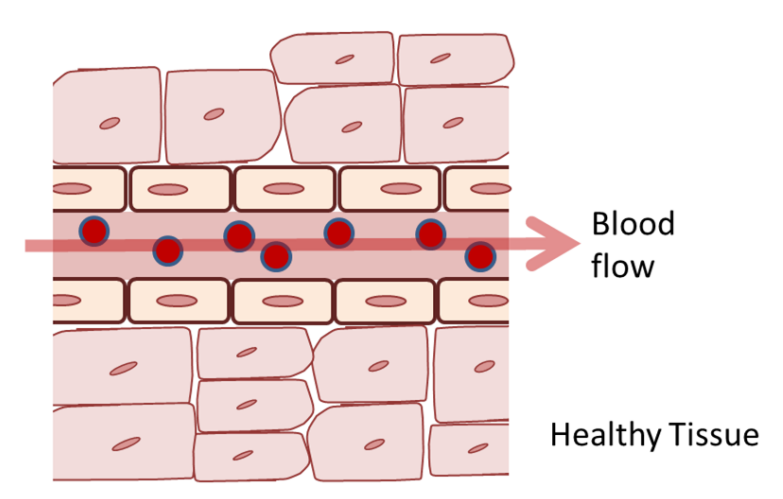

= Drug loaded TSL

$\mathrm{O}=$ Empty TSL

$\bullet=$ Released drug (b) $39-42^{\circ} \mathrm{C}$

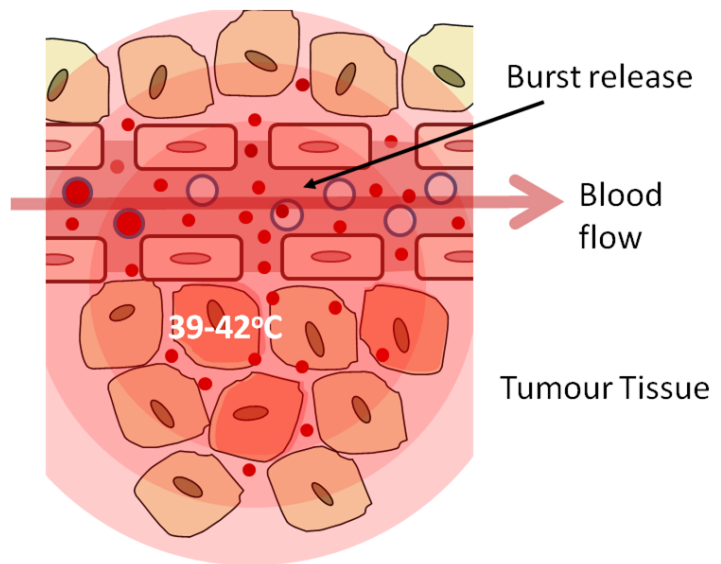

Figure 3-1: Schematic of the mode of action of a temperature sensitive liposome (TSL) for intravascular release. The TSL passes through normal unheated vasculature intact (a), but on reaching the heated tumor (b) drug is released in a burst-release fashion, creating a high local drug concentration which permeates into the tumor tissue.

Lyso-lipid Temperature Sensitive Liposome (LTSL; DPPC/MSPC/DSPE-PEG, 86:10:4 mol\%) is an example of such a burst-release TSL formulation [205], [206], which has progressed into late stage clinical trials delivering the drug doxorubicin (ThermoDox ${ }^{\circledR}$, Celsion Corporation, Lawrenceville, NJ). This formulation is currently in clinical trials for hepatocellular carcinoma (phase III), for recurrent chest wall breast cancer (phase I/II) and for liver cancer (proof-ofprinciple study). Our group has previously reported on an improved TSL formulation, HaT (ㅌeatactivated cytoToxic, DPPC:Brij78, 86:4 mol\%), which exhibited increased release of doxorubicin (DOX) relative to LTSL (2-fold at $40^{\circ} \mathrm{C}$ and 1.2-fold greater at $41^{\circ} \mathrm{C}$ ) [207]-[210]. In vivo, these release increments translated to improved tumor regression for HaT-DOX relative to LTSL-DOX. The mechanism of action for an ultrafast TSL has been investigated and discussed in the literature for LTSL-DOX [211], [212]. These studies suggest that the mechanism and biological sequelae of 


\section{CHAPTER 3}

events are different for an ultrafast TSL-DOX treatment compared to an infusion of free DOX, when each is combined with mild-hyperthermia. More pronounced activity is observed with the TSL treatment, where the rapid drug release leads to high levels of DOX in the vasculature. Subsequently, the drug attacks endothelial cells, and diffuses into the interstitial space, leading to tumor cell death. In such a case, the damaged endothelial cells may no longer offer the necessary support to contain the blood and its contents. This leads to vascular hemorrhage across the microvessel boundary, blood coagulation and vascular shutdown [207], [210]. These effects are more prominent for tumor vessels due to their inherent permeability, structural immaturity, and high proliferation in these regions of active angiogenesis [212]. Therefore, it is hypothesized that these events could be used as an endogenous physiological marker for an effective treatment and a potential surrogate marker of therapeutic effect. One such marker is the tumor blood oxygenation, and we sought to investigate this parameter in the context of relative levels of oxygen saturation $\left(\mathrm{sO}_{2}\right)$ throughout the treatment period with HaT-DOX and mild-hyperthermia.

Longitudinal monitoring of blood $\mathrm{sO}_{2}$ as a function of treatment response requires an imaging modality which can non-invasively monitor oxygenation with sufficient spatial resolution and accessibility. Optical imaging techniques are capable of measuring oxygenation, but are severely limited in their penetration depth and spatial resolution due to the dominance of ballistic photon scattering [43]. Blood Oxygenation Level Dependent contrast (BOLD) MRI and oxygen-enhanced (OE) MRI are the only clinical imaging modalities capable of assessing volumetric tumour oxygenation [213]-[215]. However, these techniques are limited by their cost and accessibility which renders them impractical for assessing the early changes in oxygenation. Photoacoustic (PA) imaging has shown a great deal of promise in combining the most advantageous features of optical modalities (contrast) and ultrasound (US) technologies (resolution) [83], [87], [190], [216]-[218]. Indeed, there are also recent reports correlating PA imaging data with that of the aforementioned MRI methods [136], [219]. PA images are acquired by detecting the ultrasonic pressure waves which are generated from the thermoelastic expansion of tissue as a result of short laser illumination. Sweeping of the optical wavelengths of illumination allows for functional PA imaging as selective absorption of the tissue chromophores would give rise to the multiple sources

of contrast contained within the PA data. In the case of oxygenation, PA imaging has been able to compute absolute values of $\mathrm{sO}_{2}$ by taking advantage of the oxygen-dependent optical absorption 


\section{CHAPTER 3}

of the hemoglobin $(\mathrm{Hb})$ inside red blood cells [160]. Photoacoustic imaging has yielded a great deal of interest over the past few years, with its ability to provide co-registered structural and functional information on a wide variety of biomedical applications. However, most approaches have focused on engineering advances with the aim of improving the spatial resolution of the technique [184]. The application of PA imaging to the treatment monitoring problem has only begun recently with several encouraging studies demonstrating the potential of the technique for detecting changes of $\mathrm{sO}_{2}$ in the tumor vasculature as a function of treatment [133] as well as during tumor development [220] and even imaging vascular perfusion [221].

Our study utilizes PA imaging to provide new insights into understanding the mechanism of action of the HaT-DOX TSL formulation and investigates the feasibility of PA imaging for cancer treatment monitoring. We utilize PA imaging to map the changes in the oxygenation of multiple slices within a murine breast cancer model in the footpad treated with our TSL formulation, HaTDOX. The efficacy of this treatment was studied relative to a saline control, and all treatments were combined with an application of mild hyperthermia (HT). Very early changes in $\mathrm{sO}_{2}$ were examined for each tumour and these were correlated to the long-term treatment outcomes.

\subsection{Materials and methods}

\subsubsection{Materials}

1,2-Dipalmitoyl-sn-glycero-3-phosphatidylcholine (DPPC) was purchased from Avanti Polar Lipids (Alabaster, AL). Brij78 [(polyethyleneglycol-20) stearyl ether], sepharose CL-4B and FITC-lectin were bought from Sigma Aldrich (Oakville, ON, Canada). DOX was purchased from Tocris Bioscience (Ellisville, MO). All other reagents were of analytical grade.

\subsubsection{Preparation of HaT-DOX liposomes}

HaT liposomes were prepared by thin lipid-film hydration followed by membrane extrusion to control size, as described in previous reports [207], [208]. Briefly, $45 \mathrm{mg}$ of lipids (DPPC/Brij, 96:4 mol\%) were dissolved in isopropanol and the solvent was evaporated under a flow of nitrogen gas at $\sim 60^{\circ} \mathrm{C}$. The resultant lipid film was dried further under high vacuum overnight to remove any residual organic solvent. Lipid films were hydrated with $300 \mathrm{mM}$ citric acid (1 mL) to form 


\section{CHAPTER 3}

multilamellar vesicles, which were then extruded 21 times through polycarbonate filters (pore size: $0.1 \mu \mathrm{m})$ at $65^{\circ} \mathrm{C}$ to adjust the liposome size. Following extrusion, formulations were cooled to room temperature and checked for size and polydispersity index (PDI) by dynamic light scattering (DLS).

To load DOX into the liposomes, a pH gradient was used to obtain a high loading of drug via a remote loading strategy. This method concentrates the drug into the liposome core through the protonation and trapping of DOX upon entering the liposome core. The $\mathrm{pH}$ gradient was generated by first exchanging the exterior buffer with HBS (25 mM HEPES buffered saline, $\mathrm{pH}$ 7.4) via dialysis (Slide-A-lyzer 10 kDa MWCO, Pierce Biotechnology, Rockford, IL). The dialysis buffer $(500 \mathrm{~mL})$ was exchanged every hour for 3 hours, at which point the $\mathrm{pH}$ was checked to ensure it was close to neutral. The liposome and DOX were then incubated at $37^{\circ} \mathrm{C}$ for $90 \mathrm{~min}$ at a $20: 1$ ratio (w/w), respectively. Following incubation, the un-encapsulated DOX was removed by purification with a sepharose CL-4B column eluting with HBS. The liposome fraction was analyzed for any change in size, PDI and drug content. DOX concentration was determined using fluorescence (excitation: $485 \mathrm{~nm}$; emission: $590 \mathrm{~nm}$ ), before and after liposome membrane disruption (Triton X-100) using a fluorescence plate reader (Hidex, Finland) as described previously [207], [208]. Particle size distributions were measured by dynamic light scattering (Zetasizer Nano-ZS, Malvern Instruments Ltd, UK). All experiments were performed with freshly prepared formulations.

\subsubsection{Cell culture and animal models}

The murine breast cancer cell line EMT-6 was purchased from ATCC (Manassas, VA). EMT-6 cells were maintained in DMEM supplemented with $10 \% \mathrm{FBS}$, penicillin $(100 \mathrm{U} / \mathrm{mL})$ and streptomycin $(100 \mu \mathrm{g} / \mathrm{mL})$ at $37^{\circ} \mathrm{C}$ with $5 \% \mathrm{CO}_{2}$. Female BALB/c mice (aged 5-6 weeks, 18-20 g) were purchased from Harlan (Mississauga, ON, Canada). All experimental protocols in this study were approved by the Animal Care Committee of the University Health Network (Toronto, ON, Canada) in accordance with the policies established in the Guide to the Care and Use of

Experimental Animals prepared by the Canadian Council of Animal Care. Mice were housed in individually ventilated cages (up to 5 mice per cage) supplied with acidified automatic watering system. Teklad irradiated rodent diet \#7912 ad lib, autoclaved corn cob bedding or iso-PADS 


\section{CHAPTER 3}

bedding was used to minimize agitation of tumors. Every cage provided with autoclaved enrichment (a translucent, red polycarbonate house and nestlets for nest building). The animal room operates at $20-22^{\circ} \mathrm{C}, 40-70 \%$ relative humidity, with a light/dark cycle of $12 / 12 \mathrm{hr}$. All animals were sacrificed with Isoflurane anesthetic followed by $\mathrm{CO}_{2}$ asphyxiation. If any signs of pain or suffering were observed, then analgesics were applied. Animals were under veterinary observation on a routine basis.

\subsubsection{In-vivo treatment protocol}

The murine breast cancer cell line, EMT-6, was inoculated $\left(1 \times 10^{6}\right.$ cells $/ 50 \mu \mathrm{L}$ medium $)$ subcutaneously into the footpad of BALB/c mice. The footpad thickness was monitored, and after $\sim 7$ days a measurable change in thickness (1.0-2.0 mm) was observed due to tumor growth. At this point, the mice were deemed ready to undergo the treatment/imaging protocol as depicted in Supplementary Figure 3.A. Following the initial pre-treatment image, each mouse was treated with one of the formulations: HaT-DOX (10 mg DOX/kg, $\mathrm{n}=13$ ) or Saline (HBS pH 7.4, $\mathrm{n}=15)$, via intravenous tail vein injection. This was immediately followed by localized heating of the tumorbearing hind limb footpad with a water bath at $43^{\circ} \mathrm{C}$ for $1 \mathrm{~h}$ (Supplementary Figure 3.A(a)). This temperature and time period was determined to be optimal for maintaining the tumor in the mildhyperthermia range in previous studies [207]-[210]. During the treatment period, mice were anesthetized with a flow of isoflurane $(1.5 \%)$ in oxygen $(0.5-1 \mathrm{~L} / \mathrm{min})$. Following treatment, the mice were returned to their cage and monitored closely to ensure full recovery and taken for imaging at further timepoints as described below. Mice were monitored regularly (every 1-2 days) for changes in footpad thickness (measured by standard calipers) and body weight. Mice were euthanized when the tumors reached double their original size (original size $=$ treatment day $=$ day 0 ) in a single dimension or reached endpoint via some other means (e.g. open tumor, 20\% body weight loss, immobility etc.). If a treatment showed a reduction in tumor size at endpoint relative to the tumor's original size at day 0 , this was defined as regression. Regression rate for a particular group was defined as the number of mice that showed regression divided by the total number of mice for each particular group. 


\section{CHAPTER 3}

\subsubsection{In-vivo imaging protocol}

Imaging of each animal was performed with the Vevo LAZR US/PA small animal imaging device (Fujifilm VisualSonics Inc., ON, Canada). This is a commercial system that consists of a 256element, $40 \mathrm{MHz}$ center frequency, linear array US/PA probe coupled to an Nd:YAG laser operated through an optical parametric oscillator with a 6 ns pulse length, $20 \mathrm{~Hz}$ pulse repetition frequency and 680-970 nm output. For the purposes of this study, the tumors were independently illuminated with 750 and $850 \mathrm{~nm}$ wavelengths. These two wavelengths were chosen to probe the optical properties of blood either side of the isosbestic point $(805 \mathrm{~nm}$, the optical wavelength at which the absorption of oxygenated and deoxygenated blood is the same). During imaging, all mice were anesthetized with a flow of isoflurane $(1.5 \%)$ in oxygen $(0.5-1 \mathrm{~L} / \mathrm{min})$. Clear ultrasonic gel was used to acoustically couple the footpad of each animal with the imaging probe, while the core body temperature was maintained at $\sim 37^{\circ} \mathrm{C}$ using a heating platform (Supplementary Figure 3.A(b)). Co-registered, 3D US and PA images were acquired by scanning the imaging probe over the entire tumor volume ( 81 frames, $80 \mu \mathrm{m}$ apart).

Imaging was performed at set timepoints before and after treatment (Supplementary Figure 3.A(c)). A pre-treatment image was taken 30 min prior to treatment for each animal. Each animal then received its dose of formulation and was immediately placed in the water bath heating set-up to receive localized mild hyperthermia (HT) to the tumor bearing hind limb as described above. Following treatment, the animal was imaged at 5 further timepoints: at $30 \mathrm{~min}, 2 \mathrm{~h}, 5 \mathrm{~h}, 24 \mathrm{~h}$ and 7 days post-treatment.

\subsubsection{Ultrasound and photoacoustic imaging and data processing}

At each imaging timepoint, a total of $21 \mathrm{US} / \mathrm{PA}, 2 \mathrm{D}$ B-mode frames (80 $\mu \mathrm{m}$ apart) were analyzed (10 on either side of the anatomical center of the tumor determined from the US B-mode image). Each 2D US image was used to anatomically segment the tumor in each frame, while avoiding the skin, bone and artifacts. The same region of interest (ROI) was applied to segment the PA images acquired at $750 / 850 \mathrm{~nm}$ for all of the 21 frames. The energy of each pulse at the two wavelengths was measured in real-time using an energy meter (Ophir-Spiricon, North Logan, Utah, USA) that was coupled to the image acquisition sequence. The PA images at each wavelength were 


\section{CHAPTER 3}

normalized by their respective, real-time energies in order to remove the wavelength-dependent laser energy variations present within the system. The PA pressure in tissue is directly proportional to the absorbed energy in tissue with the same constant of proportionality (Grüneisen parameter) throughout tissue. Given that the tumors were small and superficial, no corrections were made for the differences in tissue optical fluence for the two wavelengths. Oxygen saturation $\left(\mathrm{sO}_{2}\right)$ maps were generated by measuring the PA signal at 750/850 nm for each pixel within the tumor ROI. The $\mathrm{sO}_{2}$ was calculated based on the underlying assumption that the PA signal at the two wavelengths is primarily dominated by the optical absorption of hemoglobin $(\mathrm{Hb})$ in its

oxygenated $\left(\mu_{H b O}\right)$ and deoxygenated $\left(\mu_{H b}\right)$ forms. Eq. (3.1) shows the derivation of the $\mathrm{sO}_{2}$ from the relationship between optical absorption and chromophore concentration (oxygenated hemoglobin [HbO], or deoxygenated hemoglobin [Hb]) [222], [223],

$$
\begin{gathered}
P A_{S A}\left(\lambda_{1}\right) \propto \mu\left(\lambda_{1}\right)=[H b] \varepsilon_{H b}\left(\lambda_{1}\right)+[H b O] \varepsilon_{H b o}\left(\lambda_{1}\right) \\
P A_{S A}\left(\lambda_{2}\right) \propto \mu\left(\lambda_{2}\right)=[H b] \varepsilon_{H b}\left(\lambda_{2}\right)+[H b O] \varepsilon_{H b o}\left(\lambda_{2}\right) \\
s O_{2}=\frac{[H b O]}{[H b O+H b]}=\frac{P A_{S A}\left(\lambda_{2}\right) \times \varepsilon_{H b}\left(\lambda_{1}\right)-P A_{S A}\left(\lambda_{1}\right) \times \varepsilon_{H b}\left(\lambda_{2}\right)}{P A_{S A}\left(\lambda_{1}\right) \times \Delta \varepsilon_{H b}\left(\lambda_{2}\right)-P A_{S A}\left(\lambda_{2}\right) \times \Delta \varepsilon_{H b}\left(\lambda_{1}\right)} \\
\Delta \varepsilon(\lambda)=\varepsilon_{H b o}(\lambda)-\varepsilon_{H b}(\lambda)
\end{gathered}
$$

where, $\mu_{a}$ is the optical absorption coefficient, $P A_{S A}(\lambda)$ is the photoacoustic signal amplitude at a particular wavelength of illumination $(\lambda)$, calculated as the envelope of the time-domain PA signal within the region of interest; $\varepsilon_{H b}$ and $\varepsilon_{H b O}$ are the extinction coefficients of deoxygenated and oxygenated hemoglobin, respectively; $\Delta \varepsilon$ represents the difference in extinction coefficient between the oxygenated and deoxygenated hemoglobin. The wavelengths $\lambda_{1}$ and $\lambda_{2}$ correspond to 750 and $850 \mathrm{~nm}$, respectively.

A schematic of the algorithm used to compute the $\mathrm{sO}_{2}$ maps and histograms is shown in Supplementary Figure 3.B. In order to quantify the $\mathrm{sO}_{2}$ distribution of each tumor slice, a novel approach was employed where the $\mathrm{sO}_{2}$ intensity of all pixels within a given frame was represented in the form of a histogram. At each imaging timepoint, for each mouse, the average of 21 histograms was computed along with the standard deviation of the pixel count of each $\mathrm{sO}_{2}$ value. The resultant plot represents the temporal change in $\mathrm{sO}_{2}$ for each tumor, which were used to 


\section{CHAPTER 3}

quantify the changes in tumor $\mathrm{sO}_{2}$ as a function of time and treatment type. This approach allows for quantification of the tumor oxygenation without relying on image processing algorithms that might affect the estimated $\mathrm{sO}_{2}$. All image and signal processing were performed in Matlab2014a (The MathWorks Inc., Natick, MA).

\subsubsection{Tumor histology}

For each treatment, at least 6 mice were used to study tumor histology at two key timepoints; $2 \mathrm{~h}$ and 7 days post-treatment. These mice were randomly pre-selected for FITC-imaging prior to treatment with 3 mice being used for each timepoint of a particular treatment. Timepoints were chosen to represent both a very early timepoint post-treatment and a timepoint sufficiently late enough to begin to observe the early signs of treatment efficacy via conventional tumor measurement methods. Animals used for tumor histology were injected intravenously with FITClectin $(0.25 \mathrm{mg} / \mathrm{mL}, 200 \mu \mathrm{L})$ following their final US/PA image and returned to their cage. After $1 \mathrm{~h}$, mice were euthanized and their footpad tumors were removed and cryogenically frozen in Optimal Cutting Temperature (OCT) gel for sectioning, staining and processing by the Pathology department at the STTARR facility (Toronto, ON, Canada). Sections were stained with H\&E; fluorescent immunohistochemical stains for the vascular marker, CD31; and cell nuclei marker, DAPI. The sections were then scanned to identify the presence of FITC-lectin (green channel), cyanine dye labelled CD31 Ab (red channel) and DAPI (blue channel). Images were then processed using the Definiens software package (Munich, Germany) to quantify the intensity and distribution of each stain.

\subsubsection{Statistical analysis}

All data are expressed as mean \pm standard deviation (S.D.). Statistical analysis was conducted with the two-tailed unpaired $t$ test for two-group comparison, or one-way ANOVA, followed by the Tukey multiple comparison test by using GraphPad Prism (for three or more groups). A $p$-value of less than 0.05 was considered to be statistically significant. 


\section{CHAPTER 3}

\subsection{Results}

\subsubsection{Characterization of HaT-DOX TSLs}

The HaT-DOX liposomes were prepared as described in our earlier publications [207]-[209]. These liposomes were studied for size and drug loading to ensure all batches of liposomes possessed comparable physical characteristics (Supplementary Table 3.A). In all cases, the size observed by dynamic light scattering was within $90-100 \mathrm{~nm}$ with a PDI of $\sim 0.06$. Drug loading efficiency was generally very high $(\sim 100 \%)$ for the remote loading method used, and final drug loaded liposome concentrations were adjusted to $1 \mathrm{mg} / \mathrm{mL}$ with a drug-to-lipid ratio of $\sim 0.05$ (w/w).

\subsubsection{Tumor efficacy for a murine footpad model}

Previous studies have demonstrated improved efficacy with the HaT-DOX treatment relative to LTSL-DOX or DOX [207]-[210]. In preliminary studies, the DOX fluorescence of EMT-6 tumors was measured after treatment with equal doses $(10 \mathrm{mg}$ DOX $/ \mathrm{kg})$ of each of these 3 treatments (Supplementary Figure 3.C). HaT-DOX showed considerably greater tumoral drug uptake, and on this evidence, together with work from previous studies [207]-[210], HaT-DOX was chosen as the TSL for further study in this work. The therapeutic effect of the HaT-DOX (10 mg DOX/kg) and Saline control (sterile HBS pH 7.4) formulations were studied in a subcutaneous footpad EMT6 tumor model with mild-hyperthermia (HT) for a period of $1 \mathrm{~h}$. Tumor size and animal body weights were then followed to assess the relative efficacy of each treatment. All treatments that show a reduction in tumor size at endpoint relative to day 0 were classified as showing regression. The relative tumor sizes (\%) over the study period were plotted for each animal (Figure 3-2). It is worth mentioning that many treatments underwent a transient inflammation and swelling of the treated area for a few days post-treatment - this was particularly noticeable for many of the HTHaT-DOX treated mice (Figure 3-2a). 


\section{CHAPTER 3}
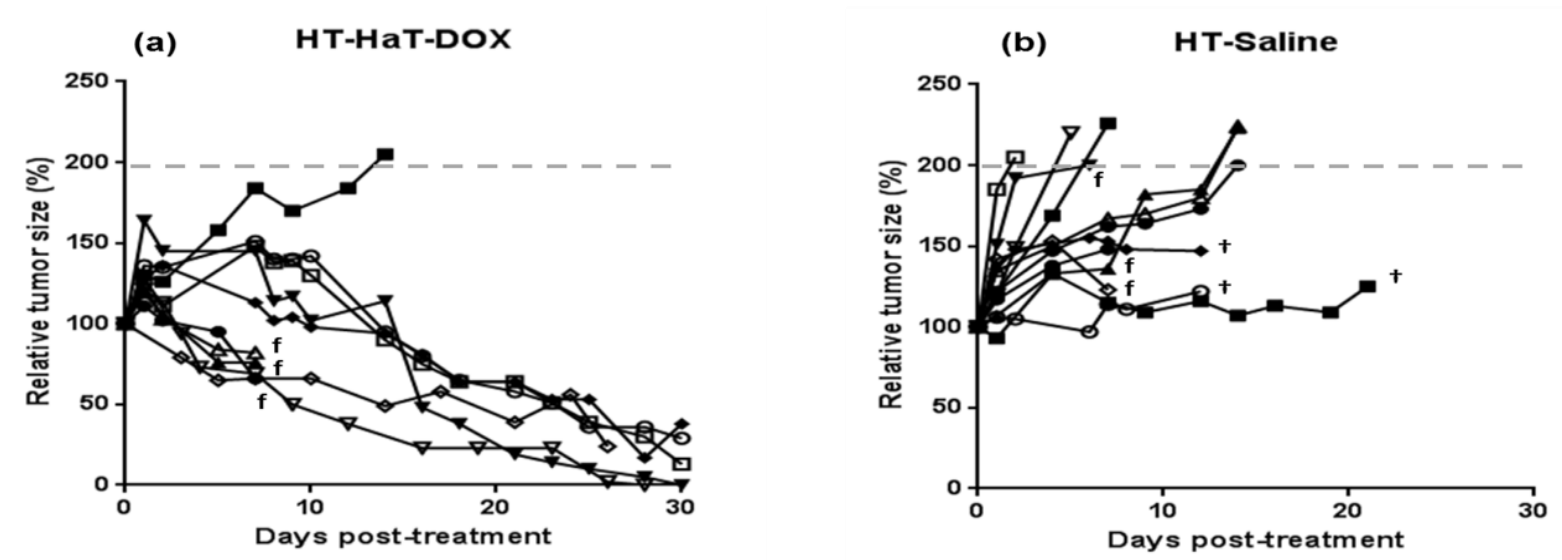

Figure 3-2: Tumor growth plots for mice treated with 1 hour of hyperthermia (HT) and an intravenous dose of (a) HaT-DOX $(n=10)$ or (b) Saline $(n=12)$. Tumors were grown subcutaneously in the footpad of the right hind limb and changes in size were measured regularly with calipers. HT-HaT-DOX treatments were dosed at $10 \mathrm{mg}$ DOX/kg. The dashed line (-----) represents the endpoint due to tumor load. The $\uparrow$ symbol indicates mice which reached a premature endpoint due to tumor ulceration or lack of sufficient mobility. The f symbol indicates all mice that were sacrificed for histology at 7 days post-treatment.

The HT-Saline treated mice may also have shown some inflammation although it was more challenging to differentiate this from tumor growth. Although there was some natural variation with each treatment, it can be seen that HT-HaT-DOX treatments generally resulted in good tumor regression (9/10 mice showed regression over 25 days), whereas HT-Saline treatments (Figure 3-2b) showed no regression, and the majority of these tumors reached endpoint by 14 days posttreatment (tumor size $200 \%$ relative to the size on day 0 ). The trends are consistent with results reported previously by our group and others for TSL and buffer control treatments combined with mild-hyperthermia [204], [208]. None of the treatments demonstrated signs of significant toxicity as represented by each of the animal's changes in body weight ( $<10 \%$ variation) during the treatment/imaging course (Supplementary Figure 3.D).

\subsubsection{Longitudinal mapping of tumor $\mathrm{SO}_{2}$}

In previously unpublished preliminary work (Supplementary Figure 3.E), the HT-HaT-DOX treatment was studied with a window chamber tumor model indicating what appeared to be localized hemorrhage and bleeding in the vicinity of the tumor. This not only highlighted to us an interest in imaging a blood dependent parameter, but also provided guidance for the selection of the appropriate timepoints for the subsequent study. Hence, the HT-HaT-DOX and HT-Saline 


\section{CHAPTER 3}

treated animals in this study were imaged at several timepoints using non-invasive, co-registered ultrasound and photoacoustic methods in order to test the hypothesis that $\mathrm{sO}_{2}$ might be a surrogate prognostic marker for effective HT-HaT-DOX treatment.

The $\mathrm{sO}_{2}$ maps derived from two-wavelength photoacoustic imaging of tumors represent the relative spatial distribution of oxygen saturation within the tumor (Supplementary Figure 3.F). A time-dependent change of $\mathrm{sO}_{2}$ was observed for the HT-HaT-DOX group and to demonstrate this visually the overall trend of the group can be represented nicely in just a few imaging timepoints from one of these animals (Figure 3-3). Each 2D map denotes the $\mathrm{sO}_{2}$ of blood inside the segmented tumor ROI at 30 min pre-treatment, and $2 \mathrm{~h}$ and 7 days post-treatment. For HT-HaTDOX treated mice, a significant drop in the tumor $\mathrm{sO}_{2}$ was observed at $2 \mathrm{~h}$ post-treatment compared to the 30 min pre-treatment image. At $2 \mathrm{~h}$ post-treatment, while some blood within the tumor still contained moderate to high $\mathrm{sO}_{2}$ values (orange/red color in Figure 3-3), the majority of the tumor exhibited very low $\mathrm{sO}_{2}$ (blue color in Figure 3-3). It is important to note that the significant drop in the tumor $\mathrm{sO}_{2}$ for the HT-HaT-DOX treated mice was apparent as early as 30 min post-treatment and it remained at these levels for more than $5 \mathrm{~h}$ (Supplementary Figure 3.F).
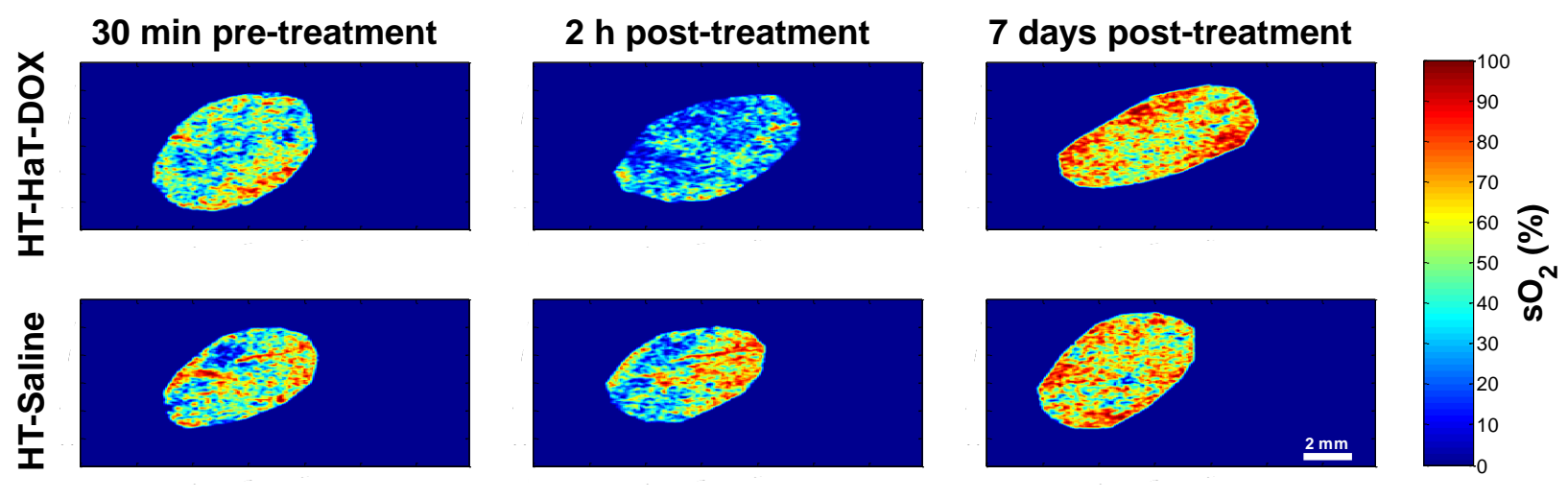

Figure 3-3: Representative sO2 maps. These are shown for HT-HaT-DOX (top row) and HTSaline (bottom row) treated tumors at 30 min pre-treatment (1st column), 2 h post-treatment ( $2 \mathrm{nd}$ column) and 7 days post-treatment (3rd column). The scale bar $(2 \mathrm{~mm})$ and sO2 color bar (0$100 \%$ ) apply to all sO2 maps shown.

The HT-Saline group received injections of HBS ( $\mathrm{pH} 7.4$ ) prior to undergoing an identical mildhyperthermia treatment protocol to the other animals that received drug formulation. $\mathrm{The}_{\mathrm{sO}}$ for 


\section{CHAPTER 3}

the HT-Saline group did not show the decrease in $\mathrm{sO}_{2}$ observed for the HT-HaT-DOX mice at $2 \mathrm{~h}$ post-treatment (Figure 3-3, bottom row). For the specific HT-Saline treated mouse represented in this figure, the $\mathrm{sO}_{2}$ shows a slight increase from the $30 \mathrm{~min}$ pre-treatment timepoint to the $2 \mathrm{~h}$ posttreatment timepoint image, as represented qualitatively by the increase in red and decrease in blue color in the image.

The trends represented in Figure 3-3 were consistent across each respective group, i.e. HT-HaTDOX treatment led to an immediate drop in tumor $\mathrm{sO}_{2}$ which was sustained for at least the first 5 h post-treatment, while for HT-Saline no such drop in $\mathrm{sO}_{2}$ was observed and levels remained relatively constant for the same period with minor fluctuations (Supplementary Figure 3.F). Upon reaching the 7-day timepoint both groups exhibited a similar behavior, where the overall $\mathrm{sO}_{2}$ rose above the level observed for their respective $30 \mathrm{~min}$ pre-treatment images.

\subsubsection{Oxygen saturation $\left(\mathrm{sO}_{2}\right)$ histograms and quantification of the changes in oxygenation}

In order to quantify the relative $\mathrm{sO}_{2}$ of the blood in tumors and capture the heterogeneity of the entire tumor volume, histograms of the distribution of $\mathrm{sO}_{2}$ values (number of pixels with a certain $\mathrm{sO}_{2}$ value as a function of that $\mathrm{sO}_{2}$ value) were calculated (Figure 3-4). The histogram of every imaging slice was combined to create an average histogram representing a treatment group at a given timepoint (30 min pre-treatment and at $30 \mathrm{~min}, 2 \mathrm{~h}, 5 \mathrm{~h}, 24 \mathrm{~h}$ and 7 days post-treatment, Supplementary Figure 3.B). 


\section{CHAPTER 3}

(a)

\section{HT-HaT-DOX}

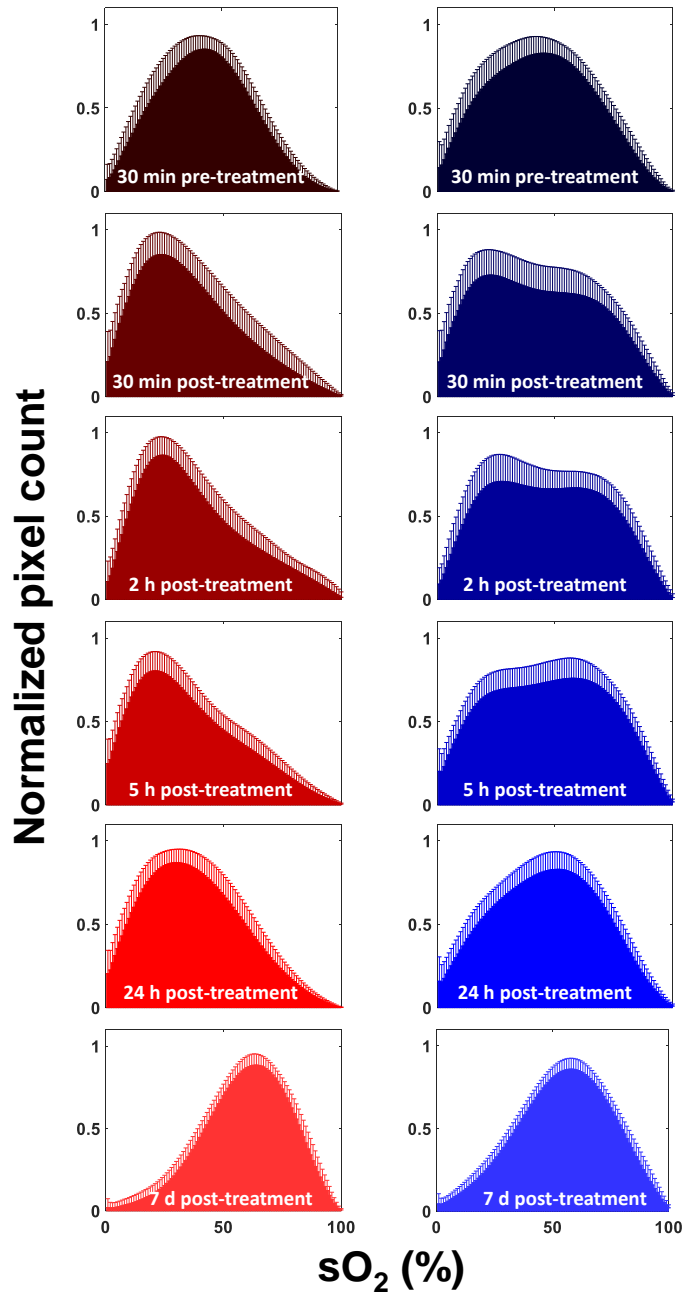

(c)
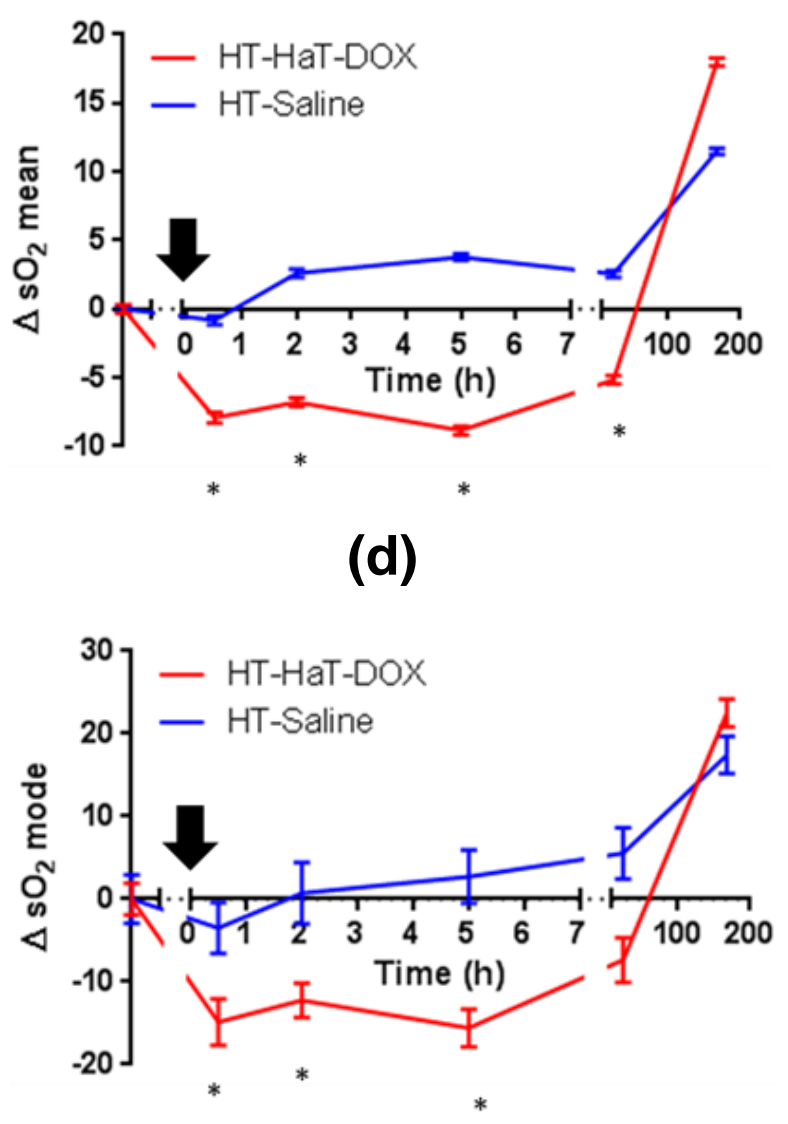

Figure 3-4:Oxygenation histograms. (a) Average $\mathrm{sO} 2$ histograms at 30 min pre-treatment and 30 min, $2 \mathrm{~h}, 5 \mathrm{~h}, 24 \mathrm{~h}$ and 7 day timepoints post-treatment for HT-HaT-DOX $(\mathrm{n}=12)$ and HT-Saline $(\mathrm{n}=15)$. Two time courses have been plotted to compare the treatments studied using the mode (b) and mean (c) averages of the histogram data plotted in (a) relative to their starting values at 30 min pre-treatment. Error bars represent the standard deviation on the pixel count for each $\mathrm{sO} 2$ value from each mouse which had 21 different histograms per imaging timepoint. The black arrows represent the points at which the treatments were made (i.e. defined in this plot as $0 \mathrm{~h}$ ). Datapoints that show a drop in $\mathrm{sO} 2$ which is significantly different to pre-treatment are represented by $*$ where $\mathrm{p}<0.05$.

Regardless of the timepoint or treatment type, all histograms showed a distribution of $\mathrm{sO}_{2}$ values. The histogram of the HT-HaT-DOX treated animals revealed a significant shift to the left during the first few timepoints, representing a drop (>15\% in histogram mode) in oxygen saturation (Figure 3-4a). This shift was apparent as early as $30 \mathrm{~min}$ post-treatment and it persisted for the 


\section{CHAPTER 3}

first $5 \mathrm{~h}$, in agreement with the $\mathrm{sO}_{2}$ map images (Figure 3-3 and Supplementary Figure 3.F). The histogram shifted to higher $\mathrm{sO}_{2}$, approaching that of pre-treatment levels by $24 \mathrm{~h}$, and by 7 days it had surpassed the values of the pre-treatment histogram. This dynamic shift in the distribution of blood $\mathrm{sO}_{2}$ values within the tumor was quantitatively represented by the early drop (30 min to $5 \mathrm{~h}$ post-treatment) and late (7 day) increase in tumor $\mathrm{sO}_{2}$ observed following HT-HaT-DOX treatment.

Histograms for the HT-Saline group exhibited comparable 30 min pre-treatment $\mathrm{sO}_{2}$ distributions to the HT-HaT-DOX group (modes $~ 40 \%$ ). However, there was no significant drop in the average $\mathrm{sO}_{2}$ (either mean or mode) during the first $5 \mathrm{~h}$ post-treatment, and again an increase was observed by 7 days post-treatment. In general, the post-treatment histograms of the HT-Saline group appeared to have broader distributions and displayed increased bimodal character (e.g. $2 \mathrm{~h}$ histogram for HT-Saline in Fig 4a), than observed for the HT-HaT-DOX group.

Given that a distribution of $\mathrm{sO}_{2}$ values exists within the tumor, one must quantify the changes in the $\mathrm{sO}_{2}$ over time by focusing on the statistics of the histograms. The mode of the $\mathrm{sO}_{2}$ within the tumor, represented by the peak of a histogram, can be representative of how the $\mathrm{sO}_{2}$ distribution varies over time, and this is particularly effective for HT-HaT-DOX, but this is less meaningful for the broad distributions for the HT-Saline treatment, particularly when bimodal character is observed. For this reason, we studied both the mode and mean $\mathrm{sO}_{2}$ change over time (Figure 3-4b and Figure 3-4c). For the HT-HaT-DOX treated mice, a significant drop in the mean $(\sim 10 \%)$ and mode $(\sim 15 \%)$ of the histogram was observed from $30 \mathrm{~min}$ pre-treatment to $30 \mathrm{~min}$ post-treatment. This is indicative of an overall shift of the entire tumor region to reduced $\mathrm{sO}_{2}$ after treatment, and is visually represented as a shift of the entire histogram distribution to the left (Figure 3-4a). The drop in the mean $\mathrm{sO}_{2}$ value was sustained for the first $5 \mathrm{~h}$. Following this initial period, a gradual increase in mean and mode $\mathrm{sO}_{2}$ for HT-HaT-DOX was observed until day 7 , reaching levels 15 $20 \%$ above those at 30 min pre-treatment.

The changes in mode and mean of the $\mathrm{sO}_{2}$ histogram for the HT-Saline group (Figure 3-4b and Figure 3-4c) were quite different to those of the HT-HaT-DOX group over time. The histogram mode showed very little change from pre-treatment during the first $5 \mathrm{~h}$ post-treatment. The mean 


\section{CHAPTER 3}

$\mathrm{sO}_{2}$ also varied much less than for HT-HaT-DOX but showed a slight increase (2-3\%) in $\mathrm{sO}_{2}$ over the first $5 \mathrm{~h}$ post-treatment relative to pre-treatment. By the 7 day timepoint, the mean $\mathrm{sO}_{2}$ had increased to $\sim 10 \%$ above pre-treatment levels.

\subsubsection{Correlation between early changes in $\mathrm{SO}_{2}$ and treatment efficacy}

The relationship between the change in tumor size (at endpoint relative to day 0 ) and the change in the mean $\mathrm{sO}_{2}$ (at $2 \mathrm{~h}$ post-treatment relative to 30 min pre-treatment) was plotted for the mice of both treatment groups (Figure 3-5). From analysis of the HT-HaT-DOX group there was a significant separation between the mice which showed regression and the one that did not. The mice that responded to treatment exhibited a decrease in tumor size of at least $50 \%$ by their endpoint and their mean $\mathrm{sO}_{2}$ at $2 \mathrm{~h}$ had dropped by an average of 10-15\% from 30 min pretreatment values. This figure demonstrates how a large drop $(>10 \%)$ in mean $\mathrm{sO}_{2}$ at $2 \mathrm{~h}$ posttreatment was typically correlated with a large tumor regression by endpoint. The majority (90\%) of animals from the HT-HaT-DOX group are contained within one standard deviation of the mean. The HT-Saline treated mice did not show such a clear trend as represented by the wide distribution of data points and larger standard deviation on both axes.

A single HT-HaT-DOX treated mouse (marked $\dagger$ in Figure 3-5) did not show the characteristic drop in $\mathrm{sO}_{2}$ of more than $10 \%$ by $2 \mathrm{~h}$ post-treatment as observed for the other HT-HaT-DOX treated animals. In fact, this treatment displayed no significant decrease in $\mathrm{sO}_{2}$ throughout the first $5 \mathrm{~h}$ post-treatment. Furthermore, no regression was observed for this particular treatment, with the tumor increasing in size to $>200 \%$ in just 7 days. 


\section{CHAPTER 3}

(a)

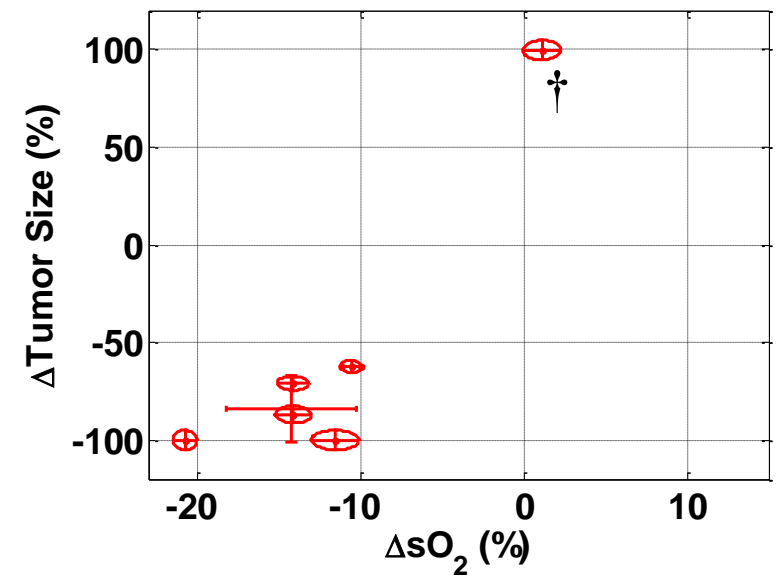

(b)

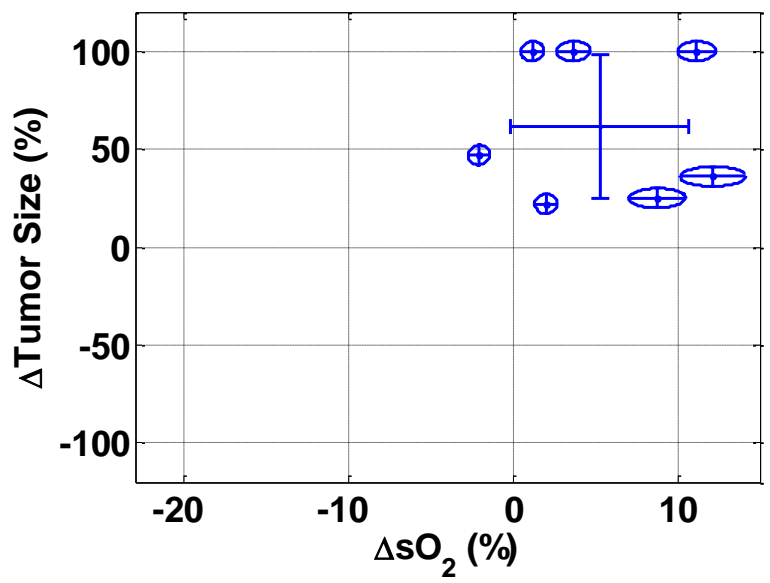

Figure 3-5: Size and oxygenation relationships. Correlation between the changes in the size of the tumor treated with (a) HT-HaT-DOX and (b) HT-Saline at endpoint (from day 0) and the changes in mean $\mathrm{sO} 2$ between the values observed for 30 min pre-treatment and $2 \mathrm{~h}$ post-treatment. Each point is the average of $21 \mathrm{sO} 2$ histograms at the $2 \mathrm{~h}$ timepoint. The major and minor axes of each ellipse represent the standard deviations of the change in $\mathrm{sO} 2$ and change in tumor size, respectively. $\dagger$ identifies a datapoint for a HT-HaT-DOX treatment that did not show regression, nor a characteristic drop in $\mathrm{sO} 2$ at $2 \mathrm{~h}$ post-treatment.

\subsubsection{Histological analysis of tumor treatment}

To investigate the cause for the observed changes in $\mathrm{sO}_{2}$, a second experiment was performed where animals were sacrificed at two distinct timepoints post-treatment ( $2 \mathrm{~h}$ and 7 days) following treatment with mild-hyperthermia and either HaT-DOX or Saline. These timepoints were chosen in order to represent the key changes observed with PA imaging. Sections of HT-HaT-DOX treated tumor showed significant FITC-lectin perfusion and leakage from the vasculature at the $2 \mathrm{~h}$ timepoint (Figure 3-6a and Figure 3-6c). This level of FITC leakage was not observed for the HTSaline group at $2 \mathrm{~h}$ or 7 days (Figure 3-6b and Figure 3-6d), nor for the HT-HaT-DOX treated mice at 7 days post-treatment. The area of FITC positive tumor was analyzed with Definiens software to provide quantitative results (Figure 3-6e and Figure 3-6f), demonstrating an average area of $\sim 60 \%$ FITC positive tumor for the HT-HaT-DOX mice at $2 \mathrm{~h}$, while only $20-40 \%$ was observed for all timepoints with the HT-Saline treated animals. The level of vessel perfusion was also studied and showed a similar trend between treatments and timepoints (Supplementary Figure 3.G). This data also correlates well with observations using the window chamber model (Supplementary Figure 3.E) with significant FITC-leakage/bleeding observed in both cases at the $2 \mathrm{~h}$ post-treatment timepoint. 


\section{CHAPTER 3}
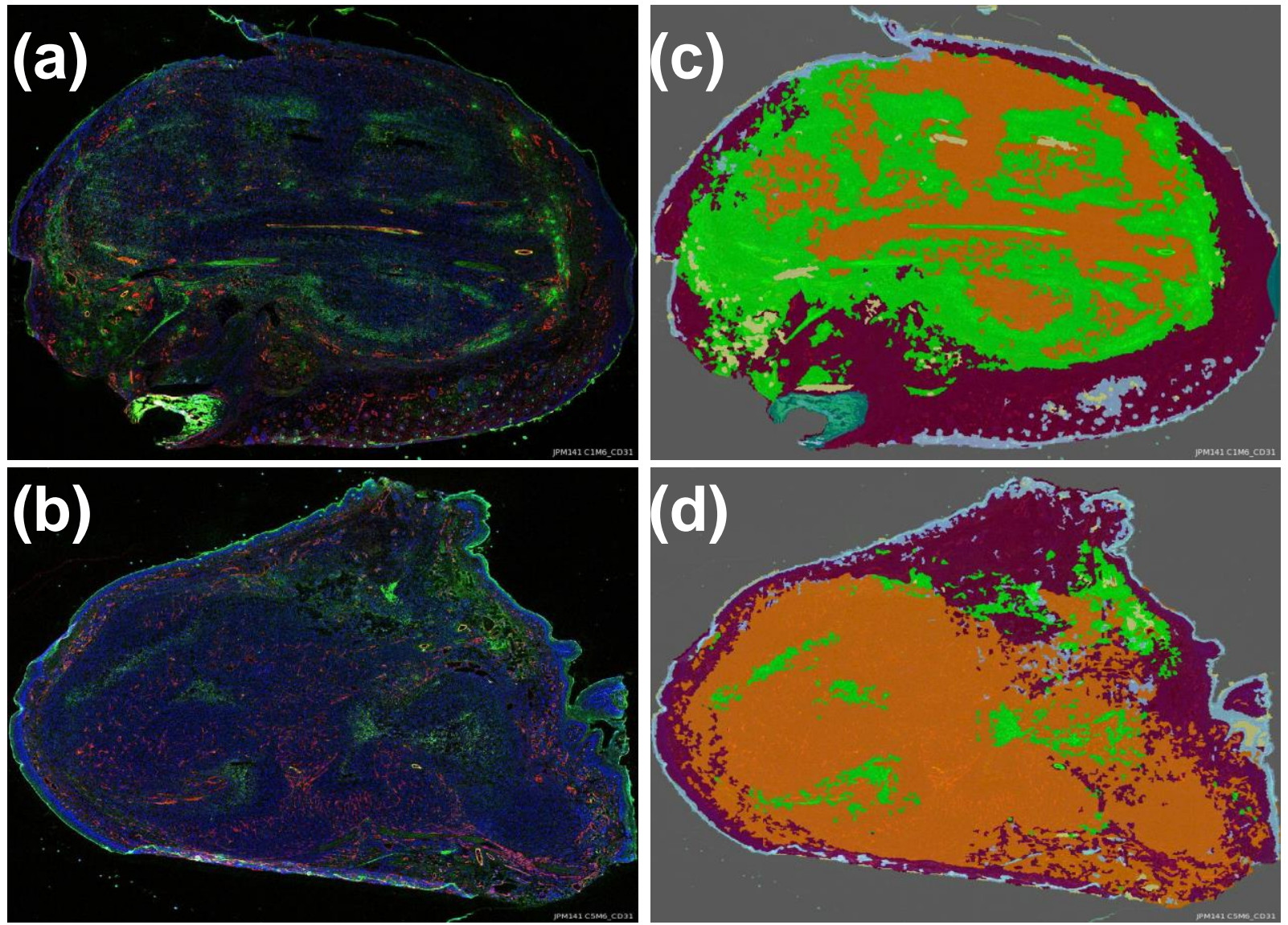

(e) $2 \mathrm{~h}$ post-treatment

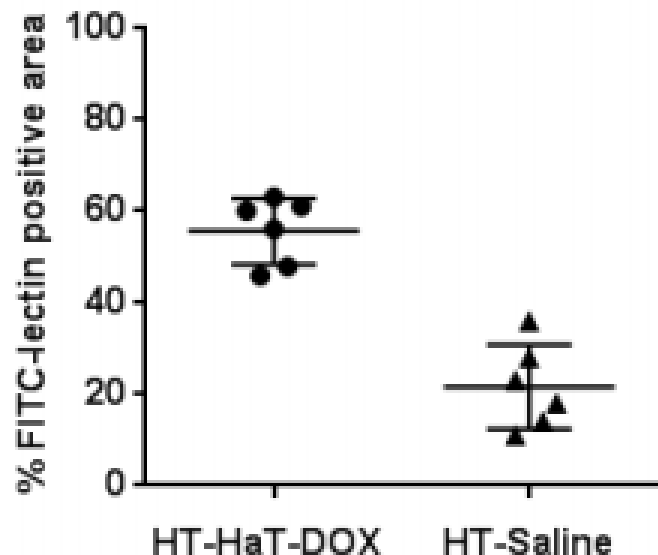

7 days post-treatment

(f)

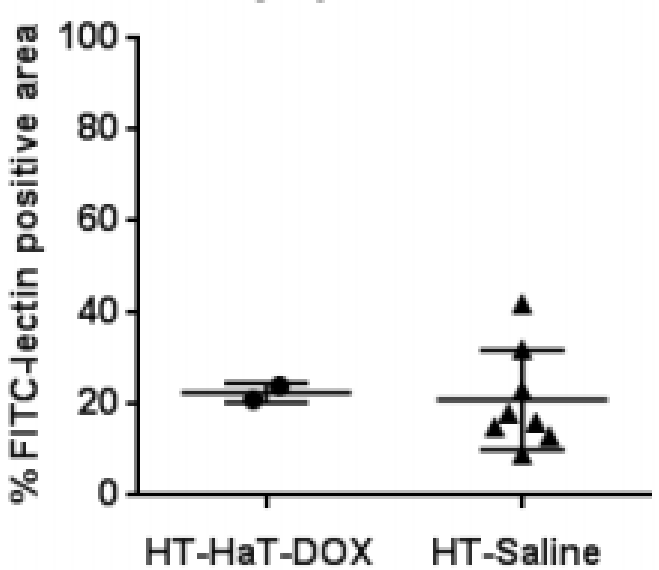

Figure 3-6: Tumor histology. Representative sections of footpad tumor harvested at the $2 \mathrm{~h}$ timepoint and stained with CD31 (red), DAPI (blue) and FITC-lectin (green) for (a) HT-HaTDOX, and (b) HT-Saline treated mice. The same sections displaying the regions defined as FITCpositive tumor (green), FITC-negative tumor (orange) and normal tissue (maroon), after processing with Definiens software for (c) HT-HaT-DOX and (d) HT-Saline. Column scatter plots: Relative FITC-positive areas following quantification with a Definiens analysis at (e) $2 \mathrm{~h}$ and (f) 7 days post-treatment; Significance is represented by $*$ where $\mathrm{p}<0.0005$. 


\section{CHAPTER 3}

\subsection{Discussion}

There is a need for chemotherapies that are localized in their action; this could provide a means of limiting toxicity to normal tissue and improve the therapeutic window of the drugs involved [224]. Triggered release nanoparticles provide one way this could be achieved [225]-[227], an example of which are TSLs [203]. Regardless of the treatment type, emerging evidence suggests that early assessment of therapeutic effect has the potential to have a significant clinical impact [43], [228][232]. The early readout of treatment efficacy could potentially be achieved directly through measurements of relative levels of endogenous biomarkers [233], [234]; an approach which is warranted by the complex nature of cancer growth and treatment response that requires personalized therapies as well as personalized means of assessing treatment outcome [234]. Even with the impressive advancements in personalized medicine and nanotechnology, current practice for assessment of cancer treatment efficacy is often limited to the anatomical information obtained through imaging studies using magnetic resonance or computed tomography. These methods can be prohibitively costly, often require the use of contrast agents with lengthy scan times, and most commonly measure the change in tumor size which may not be apparent until weeks after treatment. The motivation for this study stemmed from the desire to assess therapeutic effect shortly after treatment $(<6 \mathrm{~h})$ in order to make reasonable estimates of treatment prognosis and potential success on a personalized level.

Here, we have studied a TSL developed in our lab (HaT-DOX), designed to release its payload at mild-hyperthermia $\left(\mathrm{HT}, 39-42^{\circ} \mathrm{C}\right)$, and probed the tumor region in vivo with US-guided PA imaging throughout the course of the treatment period (from 30 min pre-treatment to 7 days posttreatment). We sought to demonstrate improved treatment efficacy with the HaT-DOX formulation over that of Saline when each was combined with mild-hyperthermia. In addition to demonstrating therapeutic effect, we also investigated the structural and functional changes taking place within the tumor during and following treatment, by using the non-invasive methods of ultrasound (US) and photoacoustics (PA).

HaT-DOX TSLs were prepared in a similar manner to that described previously [208], [209], and studied with a tumor footpad model, allowing the application of mild-hyperthermia $\left(43^{\circ} \mathrm{C}\right)$ localized to just the tumor-bearing hind limb. In doing so, drug delivery was targeted to the tumor 


\section{CHAPTER 3}

region and compared with Saline (HBS pH 7.4) control using the same mild-hyperthermia heating method (HT). HT-HaT-DOX showed good efficacy with $90 \%$ of tumors demonstrating significant regression by endpoint. Meanwhile the HT-Saline treatment was essentially ineffective, with no treatments displaying regression. These data closely match responses observed in previous reports with this formulation [208] and other related intravascular "burst-release" TSLs [235]. It is worth noting that while tumor inflammation most likely influenced absolute measurements of tumor volume in the first 3-10 days post-treatment, but this had no effect on the relative change at the study endpoint necessary to classify tumor response.

After demonstrating the differences in therapeutic effect, our study investigated the potential of US-guided PA imaging for non-invasive cancer treatment monitoring. PA imaging is relatively new to this field, but it offers a great deal of promise in being able to provide co-registered functional and structural information without any endogenous contrast [220], [236]. Within the resolution limits of our imaging system (45 $\mu \mathrm{m}$ axial, $90 \mu \mathrm{m}$ lateral), PA imaging was capable of capturing the oxygenation of the blood up to a depth of $11 \mathrm{~mm}$ at $40 \mathrm{MHz}$. At lower frequencies, PA has even been shown to map the location of vessels as deep as $40 \mathrm{~mm}$ in breast tissue [88].

The added spatial resolution at clinically relevant depths yields a distinct advantage of PA imaging over other optical methods that are limited to sub-micron depths due to ballistic photon scattering [87].

Based on preliminary window chamber data (Supplementary Figure 3.E), prior studies, and the proposed TSL mechanism of action (vide supra), it was hypothesized that the drug induced damage to the vasculature and tumor tissue could lead to the entrapment of deoxygenated red blood cells (known as blood pooling) in the perivascular space of the HaT-DOX treated tumors. These deoxygenated red blood cells would likely remain in this state until injury repair mechanisms start to regenerate the treatment area. Hence, during this period it should be possible to observe a distinct drop in oxygen saturation $\left(\mathrm{sO}_{2}\right)$ for all successful TSL treatments and this would be detectable with non-invasive PA imaging. Therefore, imaging timepoints ranging from 30 minutes to 7 days post-treatment were chosen based on the preliminary window chamber data collected, and the prior work on the mechanism of TSL drug action on the tumor vasculature [204]. 


\section{CHAPTER 3}

The potential for using the change in the $\mathrm{sO}_{2}$ as a surrogate marker for therapeutic effect following TSL treatment was investigated. Following treatment with the TSL HaT-DOX, we found a strong correlation between the early changes in the $\mathrm{sO}_{2}$ of a tumor, and the change in tumor volume in the longer-term. While the $\mathrm{sO}_{2}$ maps for both the HT-HaT-DOX and HT-Saline treated mice appeared very similar at 30 min pre-treatment, there was a clear change between the two groups at the 30 min post-treatment timepoint. At this early timepoint, the mode of the $\mathrm{sO}_{2}$ values was seen to drop significantly ( 10-20\%) for the HT-HaT-DOX treated mice, but not for the HT-Saline group; an effect that lasted for up to $5 \mathrm{~h}$ post-treatment. The drop in $\mathrm{sO}_{2}$ for the HT-HaT-DOX treatment correlated well with a significant tumor regression (90\% regression rate) 28 days posttreatment. No such drop in $\mathrm{sO}_{2}$ or tumor regression was observed for the HT-Saline treatment, indicating the significance of the HaT-DOX component. To the best of our knowledge this is the first time an imaging modality has been used to study the effect of a TSL on the tumor environment in order to predict the long-term therapeutic outcome.

The proposed mechanism for an ultrafast burst-release TSL (such as HaT-DOX) provides sufficient information to account for the significant drop in $\mathrm{sO}_{2}$ observed for the HT-HaT-DOX treated tumors between $30 \mathrm{~min}$ to $5 \mathrm{~h}$ post-treatment, as well as agreeing with the histological data obtained. Once vessels are disrupted due to the physiological effects of the HT-HaT-DOX treatment (likely more pronounced for the neo-vasculature of the tumor region), their ability to circulate oxygenated red blood cells is diminished, resulting in a drop of tumor $\mathrm{sO}_{2}$ levels (Figure 3-7). The high concentrations of DOX released inside the tumor vasculature leads to damage of both endothelial and tumor cells as the drug rapidly permeates out of the vessels and into the surrounding tissue, aided by the damaged vasculature as demonstrated by the increased levels of FITC-lectin observed for these tumors (Figure 3-6) and significant hemorrhage within the tumor (as observed in the preliminary window chamber study (Supplementary Figure 3.E). The observed low $\mathrm{sO}_{2}$ environment persists for more than $5 \mathrm{~h}$, after which a gradual increase in tumoral $\mathrm{sO}_{2} \mathrm{starts}$ to be observed. During this time, the natural repair mechanisms of the body will be prevalent, leading to recruitment of macrophages and immune cells for clean-up and regeneration of the damaged vasculature and surrounding tissue [237]. This was observed as a transient inflammation and swelling of the treated area for the first few days post-treatment. Approximately three to ten days later this inflammation had begun to subside, and it appeared the vasculature had been 


\section{CHAPTER 3}

repaired, accounting for the increase in $\mathrm{sO}_{2}$ observed at 7 days. These findings are further supported by the relatively small changes in tumor size over the first week for the HT-HaT-DOX group, which could also be explained by such inflammation and tissue regeneration.

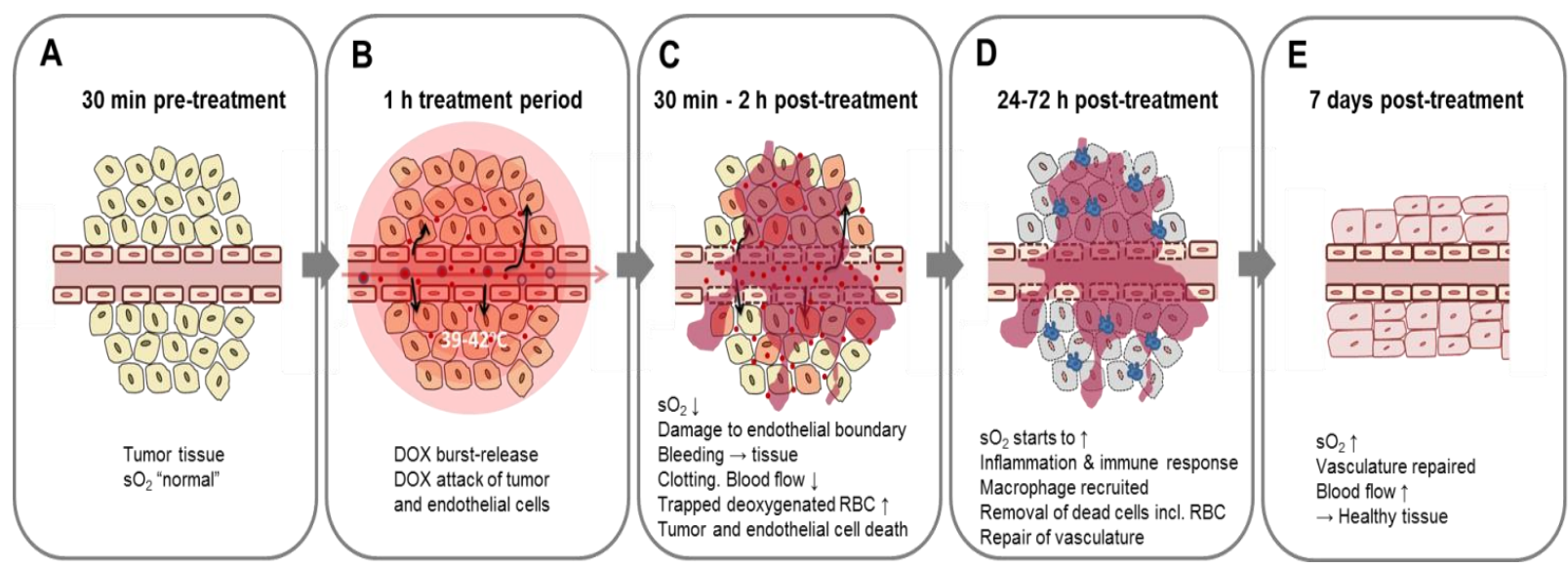

Figure 3-7: Proposed mechanism and levels of oxygen saturation (sO2) following treatment with a burst-release TSL such as HaT-DOX triggered with mild-hyperthermia. The timecourse is represented as a number of snapshots which appear sequentially from A-E.

Unlike the HT-HaT-DOX group, the HT-Saline group showed no regression, very little change in $\mathrm{sO}_{2}$ (30 min to $5 \mathrm{~h}$ post-treatment, Figure 3-4) and in most cases a significant increase in tumor size was observed. However, much like the HT-HaT-DOX group, by 7 days the HT-Saline group also showed a significant increase in $\mathrm{sO}_{2}$. In this case we speculate that the increase is due to the recruitment of new vessels required to maintain tumor growth. Consequently, the key difference observed in this study between the HT-HaT-DOX and HT-Saline groups remains the drop in $\mathrm{sO}_{2}$ observed for HT-HaT-DOX within the first 5 hours after treatment, which corresponded to the desired therapeutic effect. In future work, it would be of interest to further investigate the mechanism of action by computing the total hemoglobin concentration as a function of time. Utilizing spectral unmixing approaches [195] might elucidate the up-conversion of oxyhemoglobin to its deoxygenated counterpart and provide further evidence of vascular shutdown due to HT-HaT-DOX.

From studying the distribution of results expressed as change in tumor size (day 0 to endpoint) versus change in mean $\mathrm{sO}_{2}$ (30 min pre-treatment to $5 \mathrm{~h}$ post-treatment) a clear segregation between efficacious and non-efficacious treatments was observed. We propose that with further work a threshold $\mathrm{sO}_{2}$ drop could be identified, which would represent the "cut-off" drop in $\mathrm{sO}_{2}$ 


\section{CHAPTER 3}

required for a treatment to show significant tumor regression. For example, in the current study a single HT-HaT-DOX treated animal may have been identified as an ineffective treatment; not reaching a threshold drop in $\mathrm{sO}_{2}$ and also not demonstrating a characteristic tumor regression response (Figure 3-2 and Figure 3-5). Treatment response is affected by a wide array of factors that stem from ineffective delivery of the targeted therapeutic payload, to biological variability in the tumor's biology [238]. In the case of this HT-HaT-DOX treated animal, the tumor grew to $200 \%$ of its original size in 2 weeks, while the mean change of the $\mathrm{sO}_{2}$ histogram mean between $30 \mathrm{~min}$ and $2 \mathrm{~h}$ post-treatment was not significantly different to values recorded pre-treatment ( $p$ not less than 0.05). It is encouraging that this HT-HaT-DOX treated animal, which showed no regression, also did not reach the threshold $\mathrm{sO}_{2}$ that this work suggests is necessary for a therapeutic response. This reinforces our hypothesis that $\mathrm{sO}_{2}$ has the potential to be used as a predictor of therapeutic effect for ultrafast TSL treatments like HaT-DOX. The results of this study suggest that there is indeed added value to probing the tumor $\mathrm{sO}_{2}$ at depths that are clinically meaningful and may not be reached by means other than PA imaging.

As PA imaging begins to make its transition into the treatment monitoring arsenal, it is encouraging to see that other treatment types, (namely photodynamic therapy, antiangiogenic approaches or novel vascular strategies for augmentation of radiation therapy) are capable of inducing changes in tumor vasculature that might also be detectable with PA imaging. As Mallidi and colleagues demonstrate in their recent study, treatment response following photodynamic therapy was strongly correlated with a significant drop in $\mathrm{sO}_{2}$ several hours post-treatment [133]. Our current study builds on the findings of that work, as we demonstrate that PA imaging of oxygen saturation is able to predict the therapeutic effect of a burst-release TSL just a few hours post-treatment. We believe that our work, combined with that of others, highlights the considerable potential of PA imaging for the rapid assessment of such treatments at a personalized level.

\subsection{Conclusion}

This work provides the first example of the use of PA imaging for predicting the therapeutic effect of an ultrafast burst-release TSL treatment, though the study of endogenous $\mathrm{sO}_{2}$ values between $30 \mathrm{~min}$ to $5 \mathrm{~h}$ post-treatment. Our TSL (HaT-DOX) was studied with mild-hyperthermia (HT) and demonstrated a significantly improved therapeutic effect (regression rate of $90 \%, \mathrm{n}=10$ ), relative 


\section{CHAPTER 3}

to HT-Saline (regression rate: 0\%, $\mathrm{n}=12$ ). Simultaneously, HT-HaT-DOX and HT-Saline treatments were probed with US-guided PA imaging and a significant drop in $\mathrm{sO}_{2}(>10 \%)$ was observed for every treatment that demonstrated tumor regression by experiment endpoint (i.e. $90 \%$ of HT-HaT-DOX treatments). No such drop in $\mathrm{sO}_{2}$ was observed for any HT-Saline treatment; nor the single HT-HaT-DOX treatment that showed no regression following treatment. From this data, we suggest a threshold $\mathrm{sO}_{2}$ drop can be identified which would be necessary to achieve an effective treatment, and through further investigation, we anticipate this methodology could provide a reliable means for predicting therapeutic outcome within the first few hours of TSL treatment.

\subsection{Acknowledgments}

We would like to acknowledge the support received from the STTARR facility (University Health Network, Toronto, ON) for use of the VisualSonics VevoLAZR instrument and pathology service. We would like to acknowledge Dr. Ralph DaCosta (Princess Margaret Cancer Centre, University Health Network, Toronto) for his help with using the murine dorsal window chamber tumor model to obtain preliminary data supporting the work described in the paper (see Supplementary Material). 


\subsection{Supplementary information}

\subsubsection{Supplementary Figure 3.A}

(a)

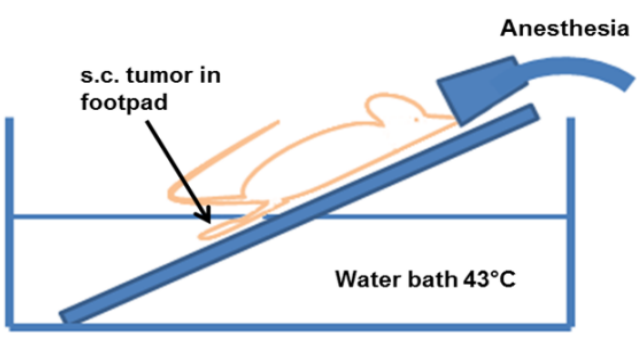

(c)

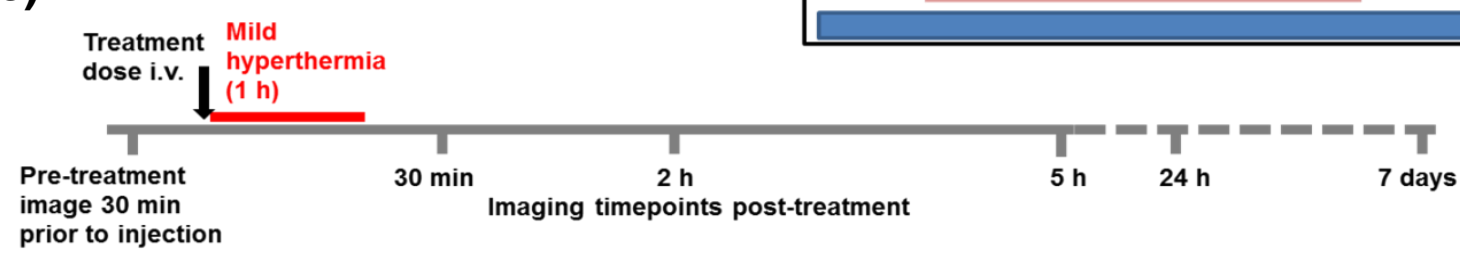

(b)

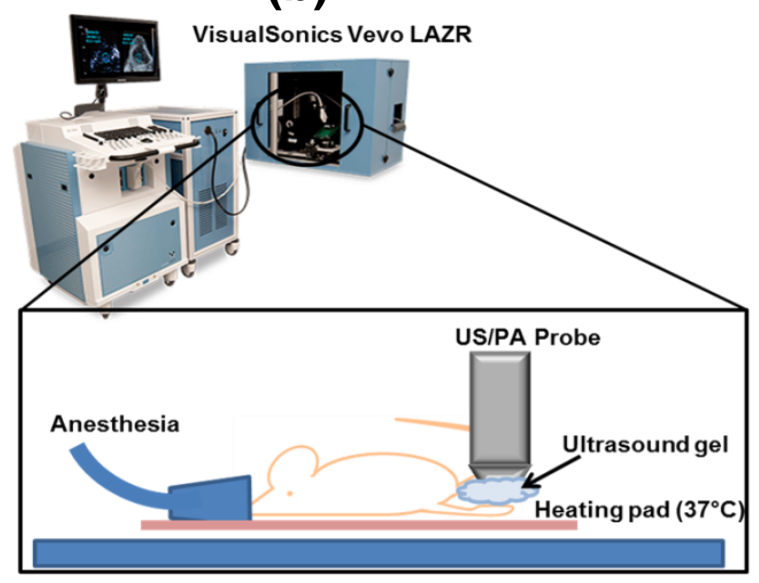

Schematic representation of the experimental set-ups. (a) The TSL treatment water bath, (b) the US/PA imaging configuration and (c) a schematic of a representative treatment and imaging time course with imaging timepoints indicated on the $\mathrm{x}$-axis.

\subsubsection{Supplementary Figure 3.B}

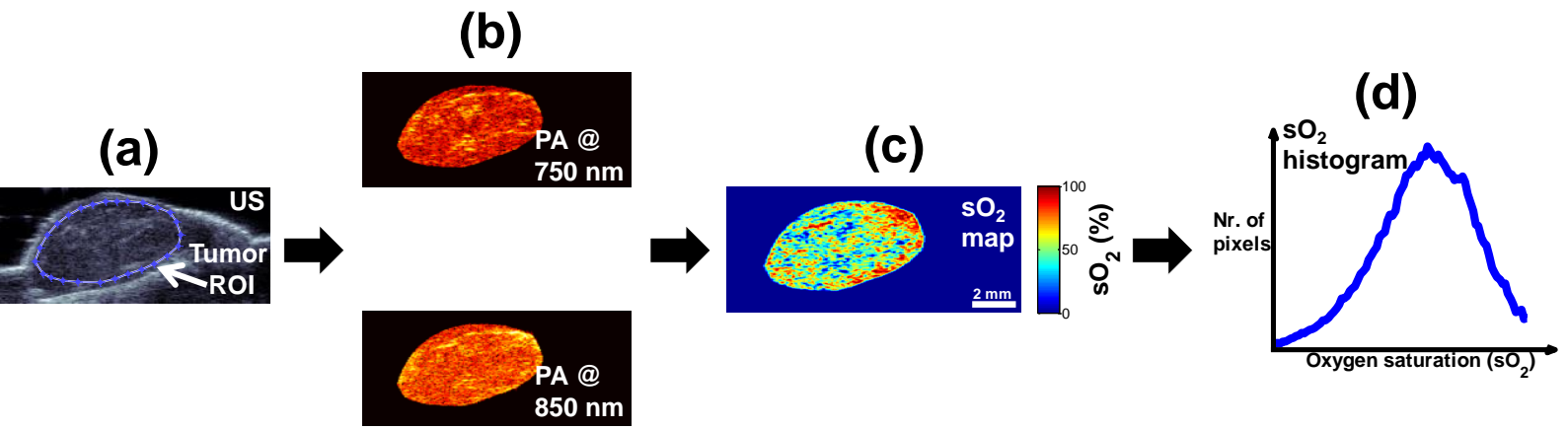

Schematic showing the process for generating tumor sO2 maps and histograms. (a) US image of a mouse footpad tumor used for anatomically segmenting the tumor ROI; (b) ROI is applied to the PA images acquired from the $750 \mathrm{~nm}$ (top) and $850 \mathrm{~nm}$ (bottom) illuminations; (c) The sO2 map is reconstructed using the algorithm described in Section 3.2.6; (d) Oxygen saturation histograms were created from the $\mathrm{sO} 2$ map data for $212 \mathrm{D}$ slices within a given tumor. 


\section{CHAPTER 3}

\subsubsection{Supplementary Figure 3.C}

\section{HaT-DOX}

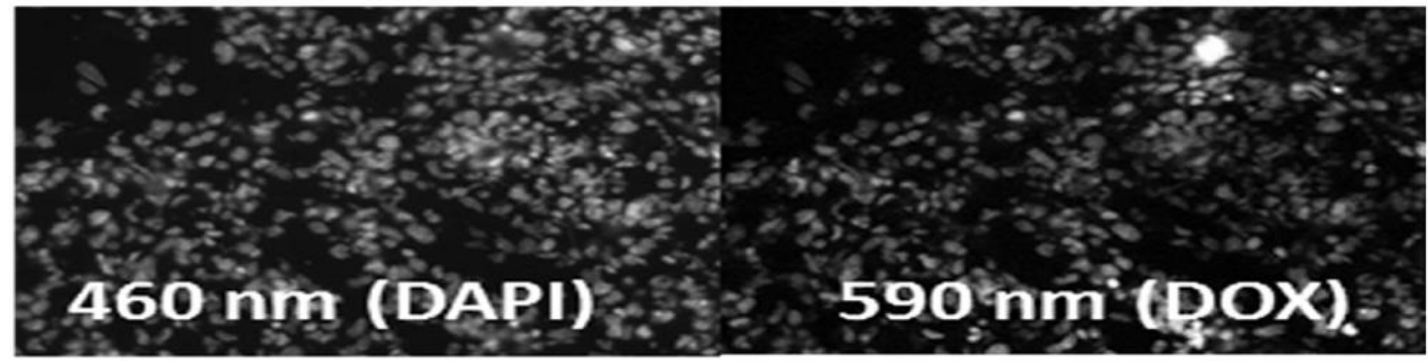

\section{LTSL-DOX}

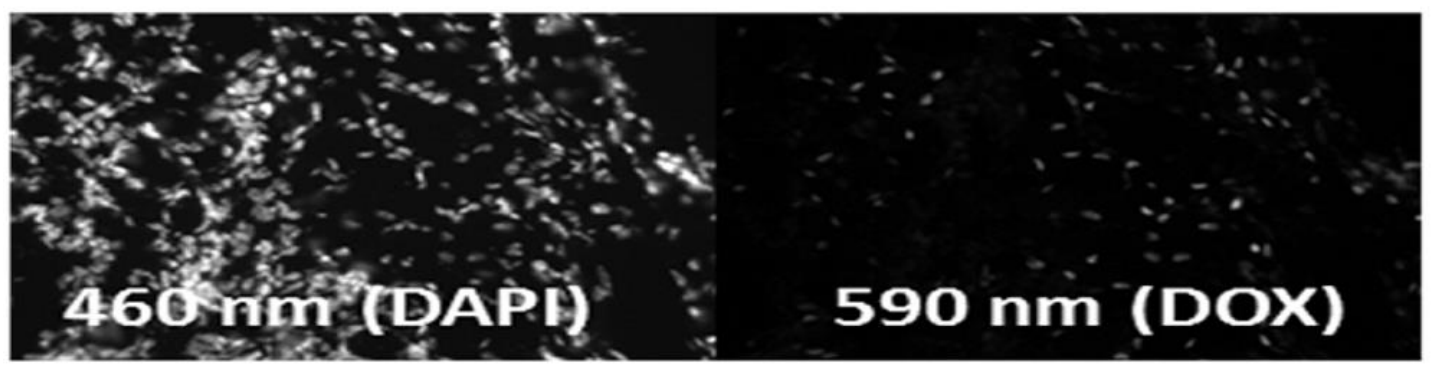

\section{DOX}

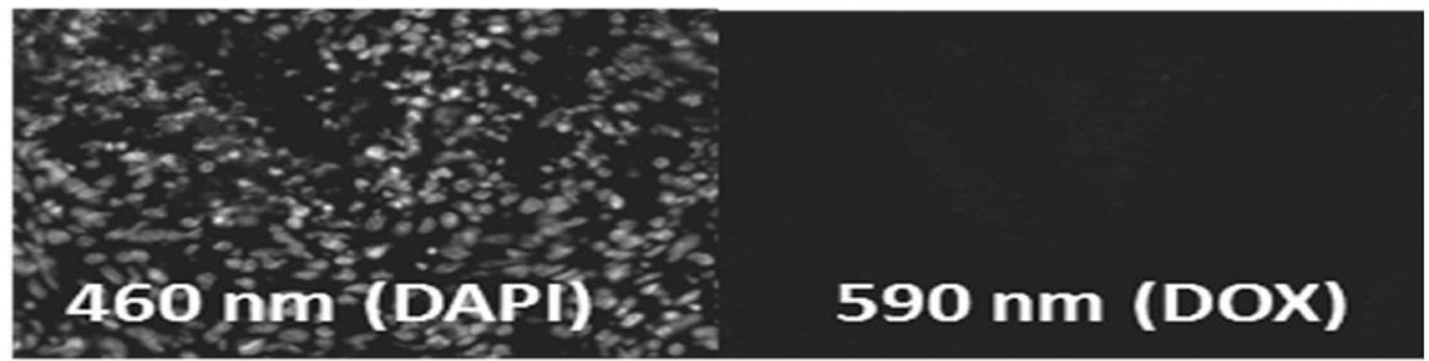

Fluorescent microscopy of tumors treated with HaT-DOX, LTSL-DOX and DOX. Each treatment dosed i.v. (10 $\mathrm{mg}$ DOX/kg) and exposed to $1 \mathrm{~h}$ of mild hyperthermia. Following this, tumors were removed, sectioned and nuclei were stained with DAPI. Sections were then studied by fluorescent microscopy to ascertain the relative amounts of DOX present in each tumor. 


\subsubsection{Supplementary Figure 3.D}

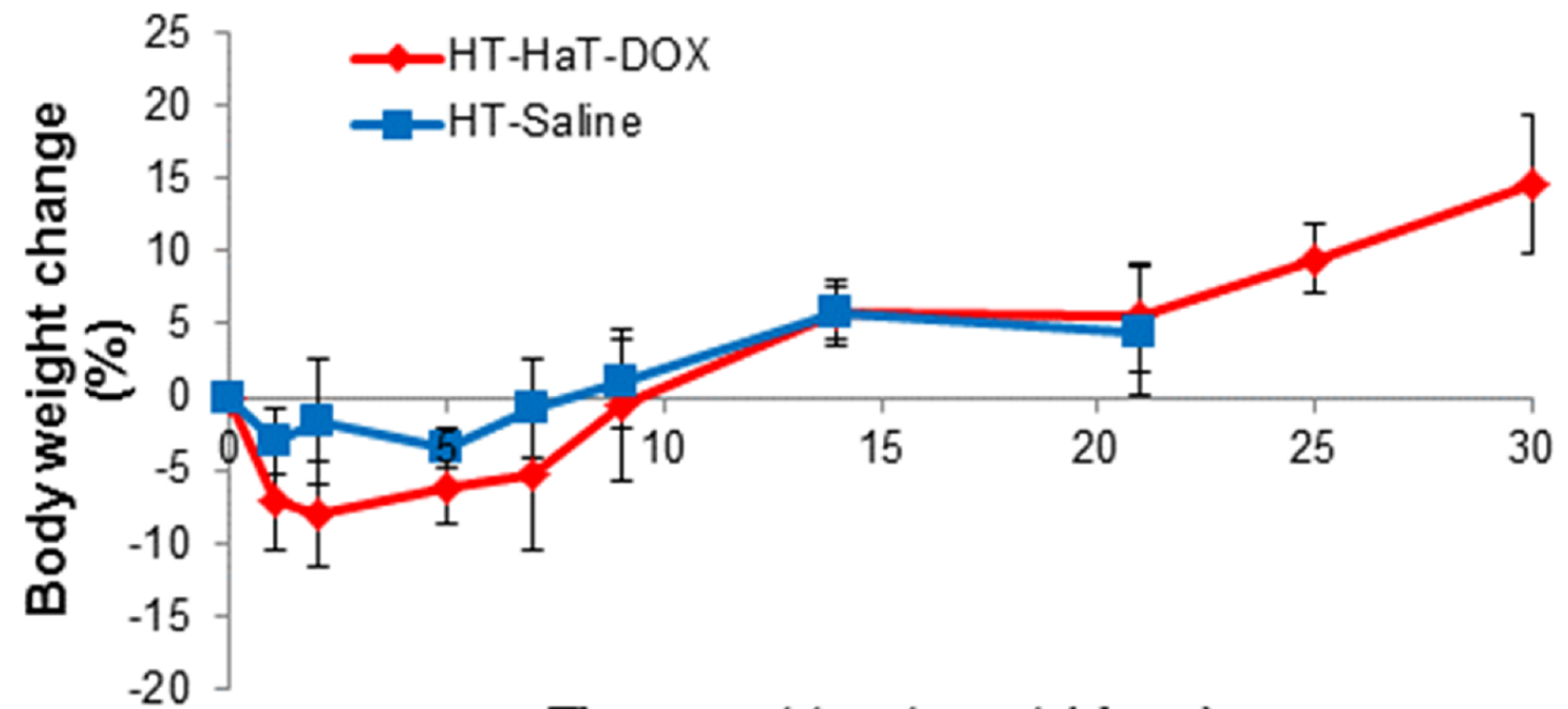

\section{Time post treatment (days)}

Body weight plot for the 2 formulations studied. Animals were dosed with either HaT-DOX or Saline and treated with mild hyperthermia $(1 \mathrm{~h})$. Data points are the average of 5 or more animals \pm S.D.

\subsubsection{Supplementary Figure 3.E}
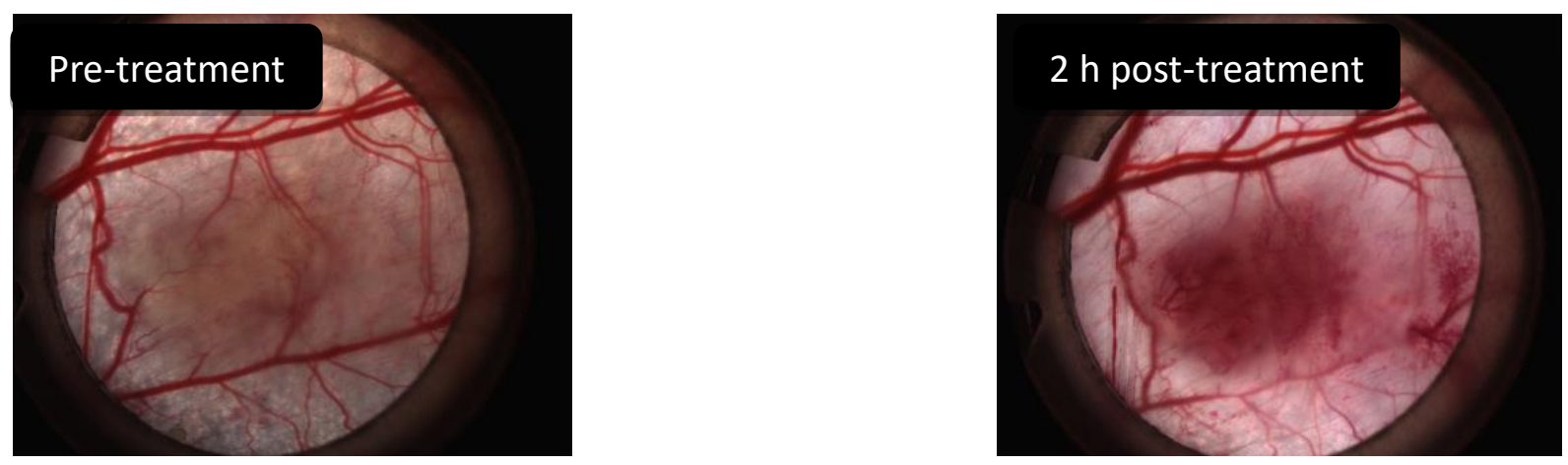

Window chamber model of HaT-DOX treatments. During our previous investigations into the HaT-DOX treatment we studied a window chamber model and observed what appeared to be localized hemorrhage and bleeding within the tumor area at $2 \mathrm{~h}$ following treatment with HT-HaTDOX. From the timepoints we studied, it appeared that this effect occurred within the first few hours post-treatment. This not only gave us good reason to explore these early timepoints posttreatment, but also suggested that suitable markers for detection of this effect could be something related to the blood-the oxygen saturation of hemoglobin ( $\mathrm{sO} 2)$ appeared to be a suitable endogenous marker, which could be studied quantitatively with non-invasive PA imaging. 


\section{CHAPTER 3}

\subsubsection{Supplementary Figure 3.F}

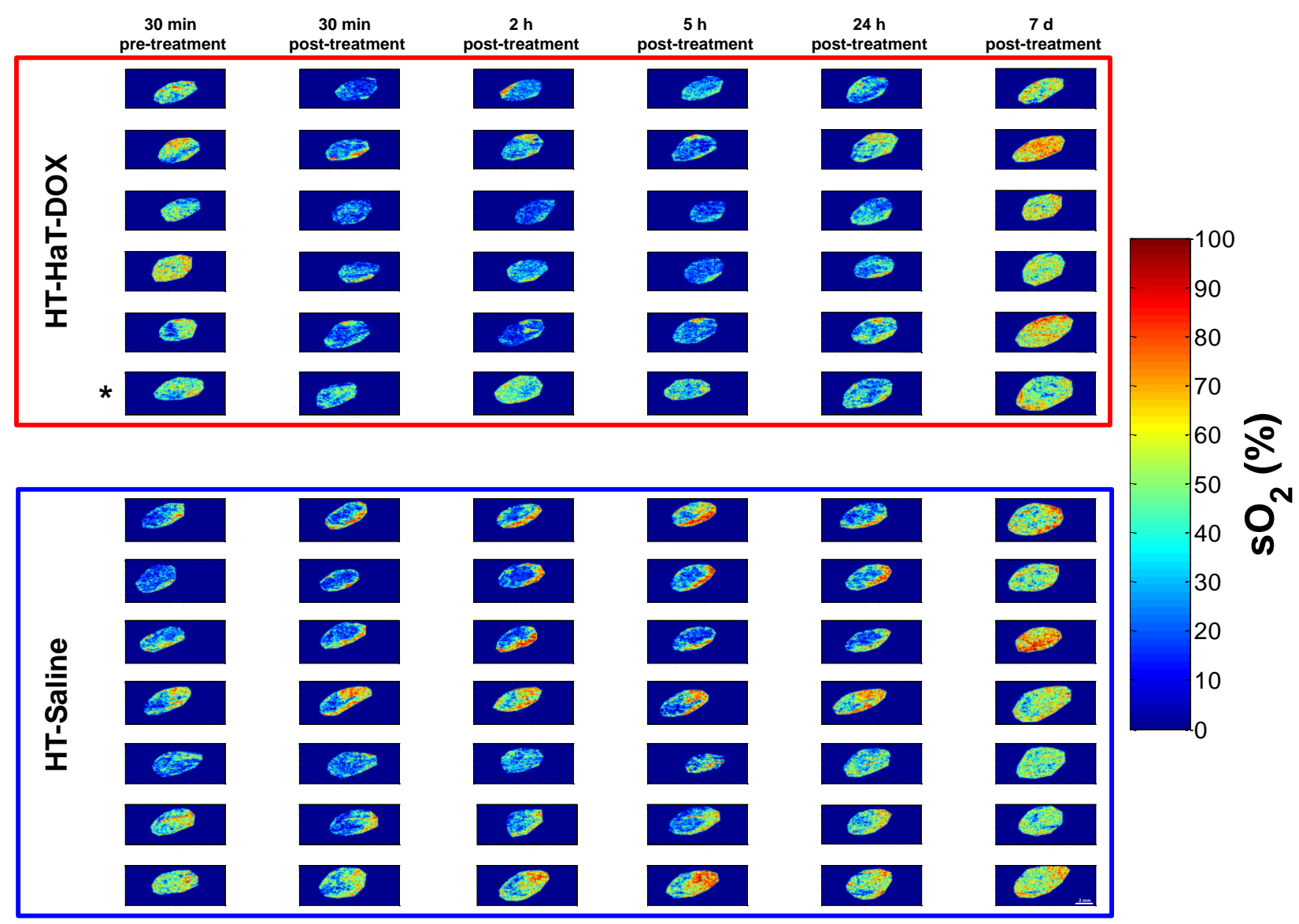

Representative sO2 maps for mice whose endpoint was greater than 7 days. The denotes the HaTDOX-treated mouse that did not respond to treatment and whose tumor grew $100 \%$ in size. The scale $(2 \mathrm{~mm})$ and color bar $(0-100 \%)$ apply to all images. 


\section{CHAPTER 3}

\subsubsection{Supplementary Figure 3.G}
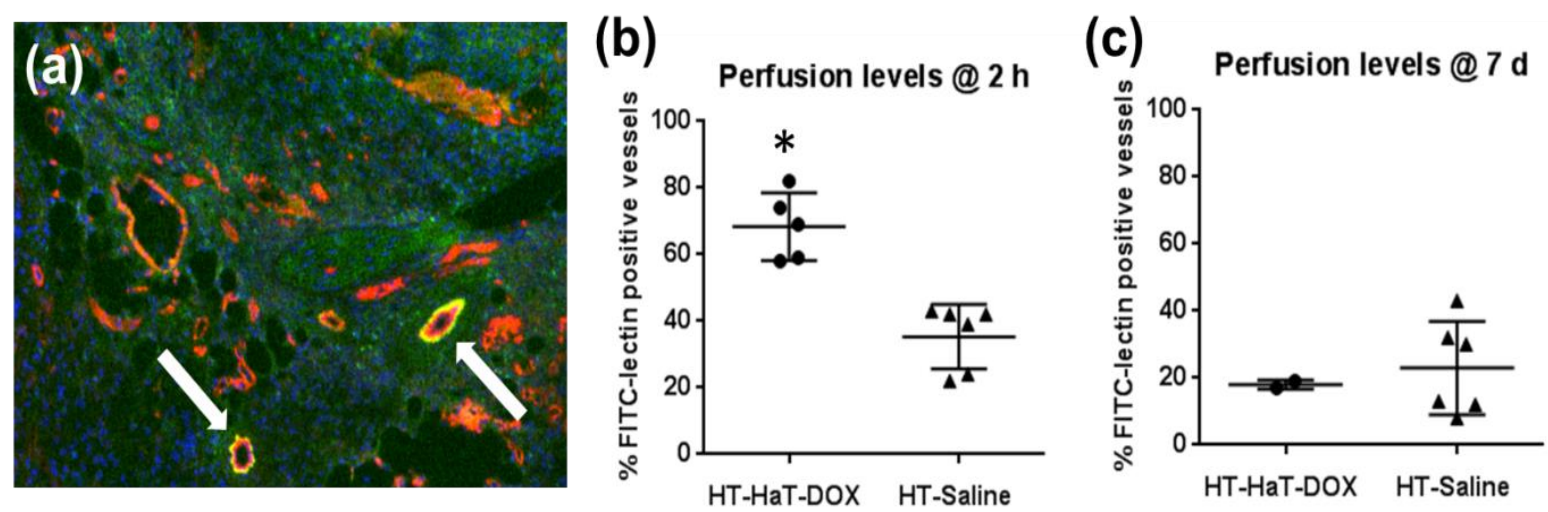

Assessments of vessel perfusion. Vessel perfusion for the HT-HaT-DOX treatment is indicated by the white arrows in the magnified image (a), where the overlap of FITC and CD31 appears yellow. Relative number of FITC-perfused vessels following quantification with a Definiens analysis at (b) $2 \mathrm{~h}$ and (c) 7 days post-treatment. Significance is represented by where $\mathrm{p}<0.0005$.

\subsubsection{Supplementary Table 3.A}

\begin{tabular}{|l|l|l|c|l|l|}
\hline $\begin{array}{l}\text { Formulation } \\
\text { name }\end{array}$ & $\begin{array}{l}\text { Z-Ave size } \\
\text { (nm) }\end{array}$ & $\begin{array}{l}\text { Polydispersity } \\
\text { Index } \\
\text { (PDI) }\end{array}$ & $\begin{array}{l}\text { Loading } \\
\text { efficiency } \\
(\%)\end{array}$ & $\begin{array}{l}\text { Drug } \\
\text { concentratio } \\
\text { n (mg/mL) }\end{array}$ & $\begin{array}{l}\text { Drug-to-lipid } \\
\text { ratio } \\
\text { (w:w) }\end{array}$ \\
\hline HaT-DOX & $91.8 \pm 1.1$ & $0.06 \pm 0.01$ & $\sim 100 \%$ & 1.0 & 0.05 \\
\hline
\end{tabular}

Physical parameters of the HaT-DOX liposomes used in this study. Values are mean \pm S.D. 


\section{Photoacoustic spectral characterization of cancer treatment response: Correlation with changes in tumor oxygenation}

The contents of this chapter have been previously published in Photoacoustics. Permission to reproduce this article was granted by Elsevier and is provided in Appendix A.1.1.

E Hysi, LA Wirtzfeld, JP May, E Undzys, S-D Li and MC Kolios. "Photoacoustic spectral characterization of cancer treatment response: Correlation with changes in tumor oxygenation" Photoacoustics 2017; 5: 25-35 - https://doi.org/10.1016/j.pacs.2017.03.003

\subsection{Introduction}

Personalized medicine initiatives aim to develop optimal cancer treatments that will direct patients towards "the right drug at the right dose at the right time" [239]. This is contingent upon not only personalized results of a highly specific/sensitive diagnostic test but also on assessing the effectiveness of the therapy post-administration. The cost of development of new drugs and treatments is estimated between $\$ 0.5-2$ billion [240]. In order for such treatments to gain enough traction, preliminary effectiveness must be assessed with cost effective and readily available imaging tools. Conventional medical imaging is already in high demand and very expensive, making the addition of treatment monitoring within existing infrastructure a challenge [6]. In addition, imaging modalities commonly used to assess treatment efficacy become even more onerous when they are used on cancer patients for whom the physical and psychological toll of the treatment prohibits multiple imaging sessions. Often, contrast agents are required causing practical limitations to have the same patient imaged at multiple imaging timepoints after treatment [7], [241]. There is a need for imaging modalities which are sensitive to early treatment-induced structural and functional changes. These technologies could generate imaging biomarkers to be used in conjunction with personalized medicine treatment regimens and therefore would provide a means for an oncologist to assess treatment response rapidly.

Quantitative ultrasound (QUS) [24], [35], [232], [242], [243] and Diffuse Optical Spectroscopy Imaging (DOSI) [26], [43], [44], [74], [244] are two promising modalities in early cancer treatment monitoring. QUS utilizes the frequency content of the ultrasound radiofrequency (US RF) signals to extract relevant information about the changes in the structural properties of cancer tumor tissue 


\section{CHAPTER 4}

during treatment [24]. 2D parametric maps scatterer size and concentration can be constructed. Ongoing clinical studies have used textural characterization of QUS spectral parametric maps to differentiate treatment response with $100 \%$ sensitivity and $93 \%$ specificity 1 week after treatment [232]. Despite its successes, QUS is only capable of measuring the structural changes of tumor cell morphology that occur during treatment. Evidence suggests that these structural changes are dependent on tumor and treatment type and may not allow this technique to be robustly extended towards all cancer types [37]. Moreover, DOSI has shown that functional and metabolic changes (ex. hemoglobin concentration) may appear at earlier timepoints in patients with locally advanced breast cancer. An oxyhemoglobin flare has been reported 24 hours post-treatment in responding patients due to the rapid increase in cellular metabolism from chemotherapeutic-induced cellular damage [43]. However, DOSI suffers from low spatial resolution rendering the anatomical localization of the metabolic changes difficult without assistance from external modalities such as MRI [244], or ultrasound and mammography [74]. Anatomical imaging must be merged with functional measures of tumor response for guiding therapy and avoiding an over-reliance on the "one-size-fits-all" conventional treatment and imaging approach.

Photoacoustic (PA) imaging has successfully mapped structural and functional aspects of biological tissue [87], [148], [217], [245], [246] such as neuronal functional connectivity [101], breast carcinoma patterns [247] and early detection of malaria [248]. Translation of PA imaging into the mainstream of clinical radiology requires well-suited clinical applications, most likely in combination with another anatomical imaging modality. Of particular importance is the similar nature of PA imaging with US whose images are naturally co-registered when the same transducers are used. Just as with the US RF signals in QUS, the frequencies of the PA signals carry information on the structure (size, shape and orientation) of the underlying absorber [124], [128], [129]. Recently published work has shown how QUS based on analysis of RF backscatter data can be used for biological tissue characterization of sub-resolution scattering structures [30]. In a similar manner, analysis of the PA radiofrequency signals has the potential of offering a system independent method of inferring sub-resolution tissue structural properties [187], [249]. As red blood cells contained within vascular tissues generate the PA signal, the quantitative photoacoustic (QPA) method would be sensitive to the spatial distribution of RBCs contained within unresolved blood vessels (or locally hemorrhaged). This added sensitivity is of importance for pushing the 


\section{CHAPTER 4}

sensitivity of acoustic resolution PA imaging beyond the resolution limit dictated by the transducer's bandwidth. Such advances would accelerate the clinical translation of PA imaging where clinically relevant ultrasound detection frequencies are used. In the past five years, several groups have employed the use of PA radiofrequency analysis to differentiate prostate adenocarcinoma tumors from normal tissue [250], detect the presence of red blood cell aggregation [187], [251], identify various stages of liver disease [249], [252] and characterize bone and joint microstructure in osteoporosis [253] and rheumatoid arthritis [254]. Theoretical models have also been developed for understanding the spectral features that arise from tissue microstructural changes [126], [139], [186], [188], [255]-[257]. Combining functional aspects of PA imaging (based on optical spectroscopy) with structural information (based on RF frequency analysis) can potentially be used to better monitor cancer treatment response.

The use of PA imaging for cancer treatment monitoring is a relatively new endeavor [133], [150], [258]. In this study, we correlate the spectral information of the PA RF data (QPA) with the oxygen saturation $\left(\mathrm{sO}_{2}\right)$ of in-vivo tumors treated with a novel thermosensitive liposome. The ultimate goal would be the development of robust quantitative imaging techniques capable of monitoring cancer treatments and predicting long term outcome. This will rely on the structural and functional changes that occur in tumor vasculature hours after the administration of treatment.

\subsection{Methods}

\subsubsection{Animal model and treatment}

Female, BALB/c mice (5-6 weeks old; 18-20 grams) were purchased from Harlan Sprague Dawley Inc. (Mississauga, Canada). The left hind footpad of each animal was inoculated with $1 \times 10^{6}$ murine breast cancer (EMT-6, ATCC, Manassas, MA) cells in $50 \mu \mathrm{L}$ DMEM medium. The tumor was grown for 7 days post-inoculation until a 1-2 $\mathrm{mm}$ increase in footpad thickness was measured. Animal protocols implemented in this study were approved by the Animal Care Committee of the University Health Network (Toronto, Canada) in accordance with the policies established by the Guide to the Care and Use of Experimental Animals (Canadian Council of Animal Care, Ottawa, Canada). 


\section{CHAPTER 4}

Treatment consisted of tail vein injections of the thermosensitive liposome containing doxorubicin (DOX) developed by our group (labeled $\underline{H}$ eat-activated cytoToxic - HaT-DOX) or control Saline. HaT-DOX preparation has been described elsewhere [204], [207]-[209]. Briefly, DOX inside the $100 \mathrm{~nm}$ liposome was rapidly (< 20 seconds) released at mild-hyperthermic temperatures (39$42^{\circ} \mathrm{C}$ ). HaT-DOX has shown improved release kinetics and tumor uptake relative to Lysolipid Temperature Sensitive Liposome (ThermoDox ${ }^{\circledR}$, Celsion Corporation, Lawrenceville, NJ), which has progressed into late stage clinical trials for hepatocellular carcinoma [206], [259].

HaT-DOX (10 mg DOX/kg) or Saline (HBS, pH = 7.4) was intravenously injected to 13 and 15 animals, respectively. The tumor-bearing footpad was then immediately placed in a water bath at $43^{\circ} \mathrm{C}$ for 1 hour under general anesthesia. The animals were separated into 3 groups based on posttreatment sacrifice timepoints: 2 hours (HaT-DOX n=3, Saline n=4), 7 days (HaT-DOX n=3, Saline $\mathrm{n}=4$ ) and beyond 7 days (HaT-DOX $n=7$, Saline $n=7$ ). Body weight and caliper measurements of the tumor thickness were recorded every few days until the study termination (28 days post-treatment) or at endpoints defined in the animal handling protocol.

Tumor histology was obtained for two imaging timepoints of interest, 2 hours and 7 days posttreatment. Prior to sacrifice, an intravenous injection of FITC-lectin $(0.25 \mathrm{mg} / \mathrm{mL}, 200 \mu \mathrm{L})$ was administered to 6 HaT-DOX treated animals and 8 Saline treated animals. This perfusion stain was allowed to circulate for 60 minutes before euthanizing the animals and surgically removing the tumors. Multiple sections were stained with H\&E and DAPI for tumor cell localization and CD31 for endothelial cell presence. The FITC-lectin was used to establish the vessel structural integrity. Image analysis and quantification of each stain was performed using Definiens software (Definiens, Munich, Germany) at the STTARR facility (University Health Network, Toronto, Canada).

\subsubsection{Longitudinal US and PA imaging}

Co-registered US and PA images of the tumors were obtained longitudinally. The Vevo LAZR system (Fujifilm VisualSonics Inc., Toronto, Canada) was used to acquire 3D images at $40 \mathrm{MHz}$ using a 256 element linear array transducer. The coaxial US cable and optical fiber bundle were integrated in a special enclosure [175]. The fiber bundle delivered wavelengths $(680-970 \mathrm{~nm})$ from 


\section{CHAPTER 4}

an optical parametric oscillator $(20 \mathrm{~Hz}$ repetition rate) coupled to a tunable $\mathrm{Nd}$ :YAG laser $(30 \mathrm{~mJ}$ per pulse, $10 \mathrm{~ns}$ pulse length). The optical fiber bundle bifurcated into two $14 \times 1.25 \mathrm{~mm}$ rectangular strips $30^{\circ}$ relative to the imaging plane. With the PA imaging mode on the system, US acquisition was synchronized with the laser output to ensure image co-registration.

Anesthetized mice were placed prone on a heating platform at $37^{\circ} \mathrm{C}$. Clear, ultrasound gel acoustically coupled the tumor with the US/PA probe. Imaging was performed at six timepoints: 30 minutes pre-treatment (Pre tx), 30 minutes post-treatment (30 min post tx), 2 hours posttreatment ( $2 \mathrm{~h}$ post $\mathrm{tx}), 5$ hours post-treatment ( $5 \mathrm{~h}$ post tx), 24 hours post-treatment ( $24 \mathrm{~h}$ post tx) and 7 days post-treatment ( $7 d$ post tx). At each timepoint, 3D US/PA data were acquired by mechanically scanning ( $80 \mu \mathrm{m}$ step size) the transducer to cover the entire tumor volume. For each step, a 2D B-mode image was beamformed using 4 laser pulses at 750/850 nm at $5 \mathrm{~Hz}$ frame rate. The energy of the beam was monitored in real time. A total of $812 \mathrm{D}$, US and PA frames were acquired for each animal at each imaging timepoint.

Reference phantoms were used to remove the system dependencies and compute spectral parameters using techniques developed in QUS [31], [260]. The US reference phantom was constructed with glass beads (diameter $6.15 \pm 1.15 \mu \mathrm{m}$, Potters Industries, Parsippany, NJ, US) in a gelatin background [261]. The top of the phantom was covered with $128 \mu \mathrm{m} \mathrm{TPX}^{\circledR}$ (Matsui Chemicals America, Inc., Rye Brook, NY, USA) for its tissue-like acoustic impedance [262]. The acoustic properties were measured using single element transducers $(1541 \mathrm{~m} / \mathrm{s}$ speed of sound; $0.723 \mathrm{~dB} / \mathrm{cm}$ attenuation at $40 \mathrm{MHz}$ ). The PA reference counterpart contained black, carbon spheres (diameter 1-12 $\mu \mathrm{m}$ ) suspended in a gelatin background with similar acoustic properties to the US phantom [176]. Both reference phantoms were imaged at the end of each day using exacty the same settings as those used for the tumors.

\subsection{3 $\mathrm{SO}_{2} / \mathrm{QUS} / \mathrm{QPA}$ signal processing}

A schematic of the signal processing that is performed using the US and PA tumor images are shown in Figure 4-1. 


\section{CHAPTER 4}

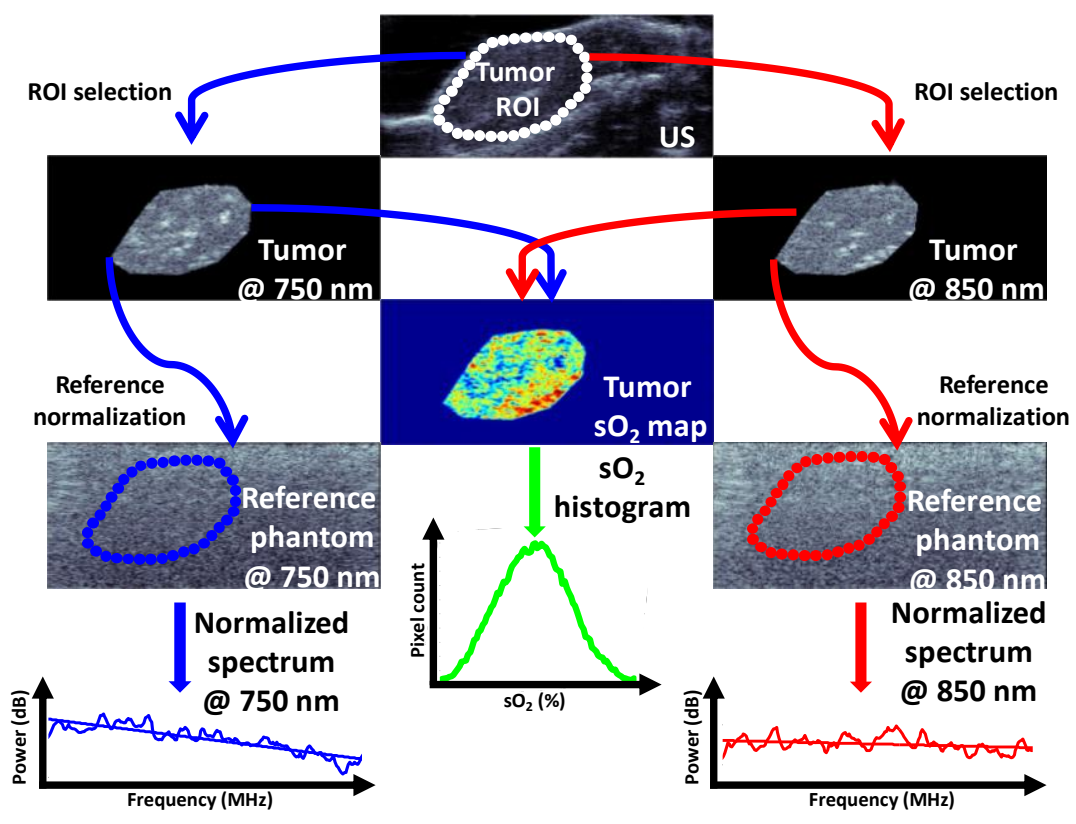

Figure 4-1: Schematic for generating tumor oxygenation maps and PA spectral parameters. The ROI segmented from the US images is applied to the co-registered PA images at 750 and $850 \mathrm{~nm}$ which are then used to generate a tumor $\mathrm{sO} 2$ map. Oxygenation histograms of every slice within the tumor provide a quantitative distribution of $\mathrm{sO} 2$ values from which the average mode is calculated. The ROI mask is also applied to the reference phantoms at both wavelengths. The frequency information of the PA phantoms is subtracted from the tumor RF spectra and the normalized power spectra are used to retrieve the spectral parameters. For the US normalized spectra, the US image and reference phantom is used to obtain the same parameters.

The US/PA datasets at every timepoint were considered to be functions of tumor spatial location, $(x, y, z)$ and optical wavelength of illumination, $\lambda$ for PA imaging. The $z$ direction refers to the transducer scanning direction over the entire tumor volume. In the temporal/spatial domain, the analysis region of interest (ROI) for the tumor was chosen by creating a spatial mask obtained by manual segmentation of the tumor for the US images:

$$
\begin{gathered}
u(x, y, z)_{\text {tumor }}=u_{0}(x, y, z) \times m(x, y, z) \\
p(x, y, z, \lambda)_{\text {tumor }}=p_{0}(x, y, z, \lambda) \times m(x, y, z)
\end{gathered}
$$

where, $u_{0}(x, y, z)$ and $p_{0}(x, y, z, \lambda)$ are the US and PA images obtained within the entire field of view of the transducer, respectively; $m(x, y, z)$ is the binary mask that contains the tumor ROI which was obtained from the manual segmentation. 


\section{CHAPTER 4}

In order to compute the oxygen saturation $\left(\mathrm{sO}_{2}\right)$ of the tumor, segmented PA images were normalized by the energy of the two laser wavelengths of illumination, 750 and $850 \mathrm{~nm}$. No fluence corrections were performed due to the complexity of accounting for the effect of wavelength dependent optical extinction on light propagation in heterogeneous tumor tissue. Here we directly use the PA amplitude ratio between $750 \mathrm{~nm}$ and $850 \mathrm{~nm}$ to relate to oxygen saturation and how these change as a function of treatment time, without compensation for local fluence variations. For this reason, changes in relative $\mathrm{sO}_{2}$ values were analyzed rather than absolute values. The effect of fluence correction on $\mathrm{sO}_{2}$ estimations has been previously reported, particularly its effect on depths greater than $5 \mathrm{~mm}$ [190], [263]. The PA signal amplitude, $p_{S A}(x, y, z, \lambda)$ was calculated

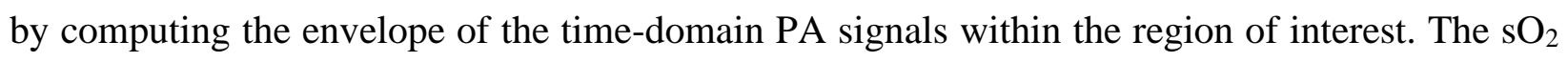
for each 2D slice was then calculated using the two-wavelength approach [223]:

$$
s O_{2}(x, y, z)=\frac{p_{S A}\left(x, y, z, \lambda_{2}\right) \times \varepsilon_{H b}\left(\lambda_{1}\right)-p_{S A}\left(x, y, z, \lambda_{1}\right) \times \varepsilon_{H b}\left(\lambda_{2}\right)}{p_{S A}\left(x, y, z, \lambda_{1}\right) \times \Delta \varepsilon\left(\lambda_{2}\right)-P A_{S A}\left(x, y, z, \lambda_{2}\right) \times \Delta \varepsilon\left(\lambda_{1}\right)}
$$

where, $H b$ refers to deoxygenated hemoglobin, $\varepsilon_{H b}(\lambda)$ is the molar extinction coefficient of deoxygenated hemoglobin at wavelength $\lambda$, and $\Delta \varepsilon(\lambda)$ is the difference between the extinction coefficient of oxygenated hemoglobin $(\mathrm{HbO})$ and its deoxygenated counterpart at $\lambda$. In this case, $\lambda_{1}$ and $\lambda_{2}$ correspond to 750 and $850 \mathrm{~nm}$, respectively. Once the $\mathrm{sO}_{2}$ value for every point in the images was computed, histograms of the distributions of $\mathrm{sO}_{2}$ values, $\mathrm{H}\left(\mathrm{sO}_{2}\right)$ were calculated. The average mode (i.e. most commonly occurring $\mathrm{sO}_{2}$ value) was retrieved for all segmented slices within the tumor and all mice at that particular timepoint and treatment group,

$$
\operatorname{Mode}\left[s \mathrm{O}_{2}\right]=\frac{1}{M S} \sum_{i=1}^{M} \sum_{j=1}^{S} \bmod \left(H_{i, j}\left(s \mathrm{O}_{2}\right)\right)
$$

where, $M$ and $S$ represent the number of mice and slices, respectively.

To compute the US and PA spectral parameters as a function of time post-treatment, the average, normalized power spectrum ( $\mathrm{PS}_{\text {norm}}$ ) was computed by removing the system dependencies through the use of the reference phantom technique developed for QUS [242]: 


\section{CHAPTER 4}

$$
P S_{\text {normUS }}(f, z)=\frac{1}{S} \sum_{i=1}^{S} \log _{10}\left(\frac{U\left(f, z_{i}\right)_{\text {tumor }}}{U\left(f, z_{i}\right)_{\text {ref }}}\right)^{2}
$$

where, $U(f, z)_{\text {tumor }}$ and $U(f, z)_{\text {ref }}$ are the average, $z-t h$ slice US power spectra for the tumor and US reference phantom in the axial dimension and averaged in the lateral dimension (at least 100 RF lines per segmentation, depending on the size of the tumor). Similarly, the average PA power spectra for the tumor and the PA reference phantom can be written as:

$$
P S_{\text {normPA }}(f, z, \lambda)=\frac{1}{S} \sum_{i=1}^{S} \log _{10}\left(\frac{P\left(f, z_{i}, \lambda\right)_{\text {tumor }}}{P\left(f, z_{i}, \lambda\right)_{\text {ref }}}\right)^{2}
$$

where, $P(f, z, \lambda)$ is the Fourier Transform of the PA signals for the tumor and reference.

The US and PA normalized spectra were fitted to a straight line within the $-6 \mathrm{~dB}$ bandwidth of the transducer (25-40 MHz in this case) in order to extract the spectral parameters [28], [29]. The spectral slope was extracted from:

$$
P S_{f i t}(f)=S S \times f+Y_{i n t}
$$

where, $P S_{f i t}$ is the result of performing linear regression on either $P S_{\text {normuS }}(f, z)$ or $P S_{\text {normPA }}(f, z, \lambda), S S$ is the spectral slope measured in $\mathrm{dB} / \mathrm{MHz}$ and $Y_{\text {int }}$ is the $\mathrm{y}$-intercept of the fit measured in $\mathrm{dB}$. An additional parameter, the midband fit (MBF), a measure of scattering strength, was assessed by measuring the power spectrum amplitude in the middle of the bandwidth used for the analysis of the signals. The goal was to extract QUS/QPA parameters that could be used to monitor the structural aspects of the HaT-DOX treatment, namely changes in the size and concentration of optical absorbers. 


\section{CHAPTER 4}

\subsection{Results and discussion}

\subsubsection{Treatment efficacy}

The progression of tumor size after the administration of the HaT-DOX and Saline treatments is shown in Figure 4-2. Each animal treated with HaT-DOX was classified as a responder if the tumor size showed a relative decrease of $>50 \%$ at study termination [264]. An overall reduction in tumor size was observed for all HaT-DOX treated animals except a single non-responder which exhibited a $100 \%$ increase in tumor size 21 days post-treatment. This non-responder mouse was treated in an identical fashion and was included in this data set as its oxygenation and QUS/QPA behavior was significantly different from HaT-DOX responders. As reported in our previous study for a larger subset of animals, a 90\% regression rate was observed 25 days after HaT-DOX treatment [150].

Thermosensitive liposomes eliminate the systemic toxicity associated with chemotherapeutic drugs such as DOX [204]. The effectiveness of the HaT-DOX treatment was evident when compared to the Saline control group, whose tumors increased $200 \%$ in size. For the first 10 days post-treatment, the behavior of the two groups was similar. Although not independently assessed, this was likely due to transient inflammation of the footpad upon DOX release [206]. As the inflammation subsided, rapid tumor regression was observed in the HaT-DOX group and by 28 days, normal footpad appearance and function (e.g. gripping) was restored. This is consistent with previous studies where the efficacy of HaT-DOX has been studied extensively [204], [208]. The variation in tumor size for the Saline group was significantly larger than for HaT-DOX. This could be a result of the random distribution of blood vessels in untreated tumors which leads to variable tumor growth rates [265] and arises as a result of the lack of DOX-induced vascular shutdown [266]. 


\section{CHAPTER 4}

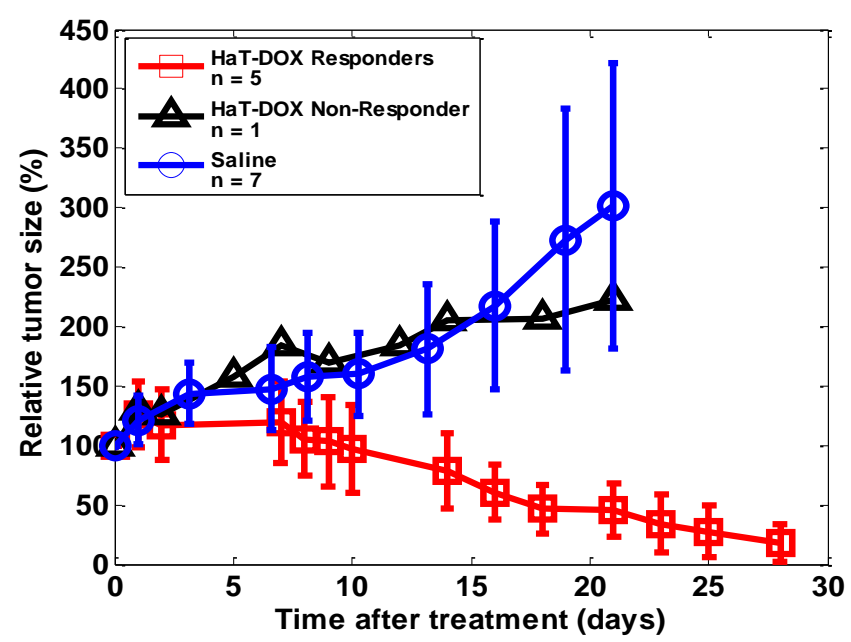

Figure 4-2: Average, relative tumor growth as a function of time post-treatment. Each point represents the average and standard deviation (error bars) of the relative tumor size for 6 HaTDOX treated mice and 7 Saline control mice. The HaT-DOX non-responder and the Saline control animals were euthanized at day 21.

\subsubsection{HaT-DOX tumor oxygenation and PA normalized power spectra}

In order to examine the changes in the oxygenation of the tumor vasculature after the administration of the HaT-DOX formulation, average $\mathrm{sO}_{2}$ histograms were plotted for the pretreatment and at the $2 \mathrm{~h} / 24 \mathrm{~h} / 7 \mathrm{~d}$ post-treatment imaging timepoints (Figure 4-3a). These animals all responded to the HaT-DOX treatment (as assessed by caliper measurements) and experienced a $2 \mathrm{~h}$ post-treatment, $22 \%$ drop in oxygenation (as assessed by the $\mathrm{sO}_{2}$ mode). This drop in oxygenation was present as early as 30 min post-treatment and was sustained for the first $24 \mathrm{~h}$. Tumor oxygenation histograms can be used to quantify changes in blood vessel oxygenation throughout the entire tumor [150]. The early changes in blood vessel oxygenation correlate with treatment response: responders' $\mathrm{sO}_{2}$ decreased 30 mins post-treatment.

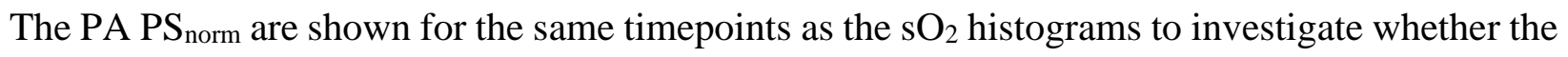
change in oxygenation is accompanied by a physical change in vascular structure (Figure 4-3b). At the middle of the transducer bandwidth (i.e. midband fit or MBF), an increase of $6.2 \mathrm{~dB}$ is observed for the $750 \mathrm{~nm}$ PS $_{\text {norm }}$ at the $2 \mathrm{~h}$ timepoint relative to pre-treatment; at $850 \mathrm{~nm}$ this increase was $2 \mathrm{~dB}$. These changes persisted for the first $5 \mathrm{~h}$ post-treatment. By $24 \mathrm{~h}$ post-treatment, the $\mathrm{PS}_{\text {norm }}$ 


\section{CHAPTER 4}

amplitude approached pre-treatment levels, and by $7 \mathrm{~d}$ post-treatment it decreased by $3.4 \mathrm{~dB}$ relative to the value at $2 \mathrm{~h}$ for $750 \mathrm{~nm}$.
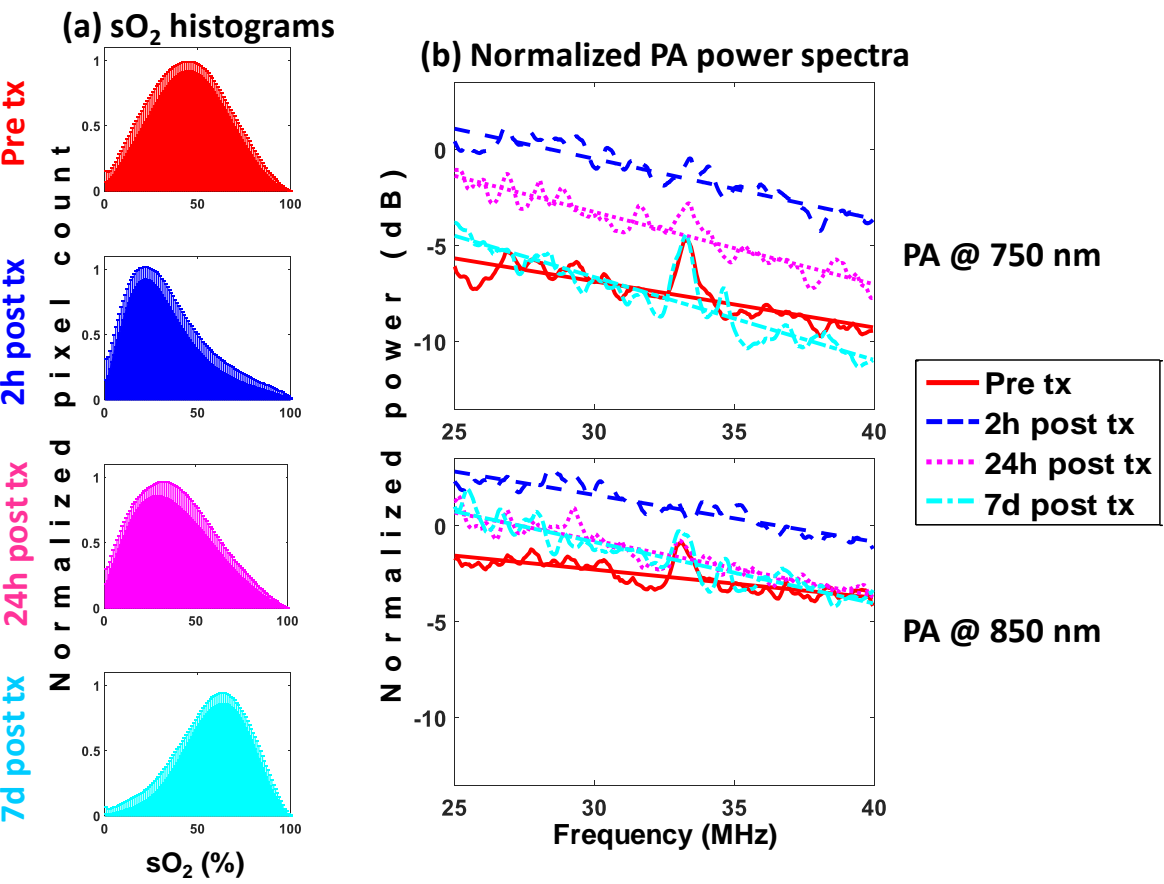

Figure 4-3: HaT-DOX-treated mice (a) tumor oxygenation histograms and the (b) normalized power spectra for PA images obtained at 750 (top) and $850 \mathrm{~nm}$ (bottom) for multiple imaging timepoints. The error bars on the histograms represent the standard deviation on the pixel count for each sO2 value obtained from 21 different histograms per timepoint per mouse. The power spectra are averages of at least $100 \mathrm{RF}$ lines per 21 tumor slices per timepoint ( 6 total) per mouse (5 total). All linear regressions had a Chi-squared value $\geq 0.9$. Treatment is abbreviated as tx.

Changes in the MBF are known to correlate to changes in the concentration of optical absorbers [187], [250], [267]. Differences in signal strength between the two wavelengths arise from the changes in vessel oxygenation. Treatment-induced vascular collapse leads to the hypothesis that oxygenation decreases at the early timepoints. Therefore, it is expected that the PA signal at 750 $\mathrm{nm}$ would be higher than for $850 \mathrm{~nm}$, as predicted from the optical absorption spectra of oxygenated and deoxygenated hemoglobin. By $7 \mathrm{~d}$ post-treatment, the $\mathrm{sO}_{2}$ of the blood vessels increased by over $20 \%$ compared to pre-treatment (Figure $4-3 \mathrm{a}$ ). This resulted in the $7 \mathrm{~d}, 750 \mathrm{~nm}$ $\mathrm{MBF}$ decrease suggesting that the MBF can differentiate between two different states of vessel oxygenation. 


\section{CHAPTER 4}

\subsubsection{Saline tumor oxygenation and PA normalized power spectra}

The $\mathrm{sO}_{2}$ histograms for the Saline-treated mice are shown in Figure 4-4a. Unlike their HaT-DOX counterparts, no significant changes in oxygenation were measured. The $\mathrm{sO}_{2}$ histograms at $2 \mathrm{~h}$ posttreatment appear wider than pre-treatment but the histogram mode did not change. This could be due to significant variability within the oxygenation and tumor size (Figure 4-2). Mice with a low pre-treatment $\mathrm{sO}_{2}$ did not undergo changes post-treatment, while others increased. At the $7 \mathrm{~d}$ timepoint, the average oxygenation of the tumor increased by $20 \%$ relative to oxygenation values pre-treatment.

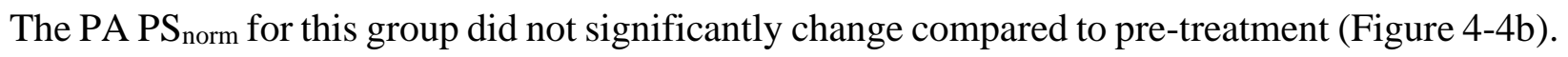
At 7d, the $750 \mathrm{~nm}$ MBF decreased by $3.2 \mathrm{~dB}$. The lack of changes in the early timepoints suggested that the Saline treatment did not induce the same vascular changes in the tumor as HaT-DOX. The decrease of the MBF at $7 \mathrm{~d}$ is correlated to a $20 \%$ increase in the $\mathrm{sO}_{2}$ of blood vessels thought to occur due to the recruitment of vessels required to maintain tumor growth [265].

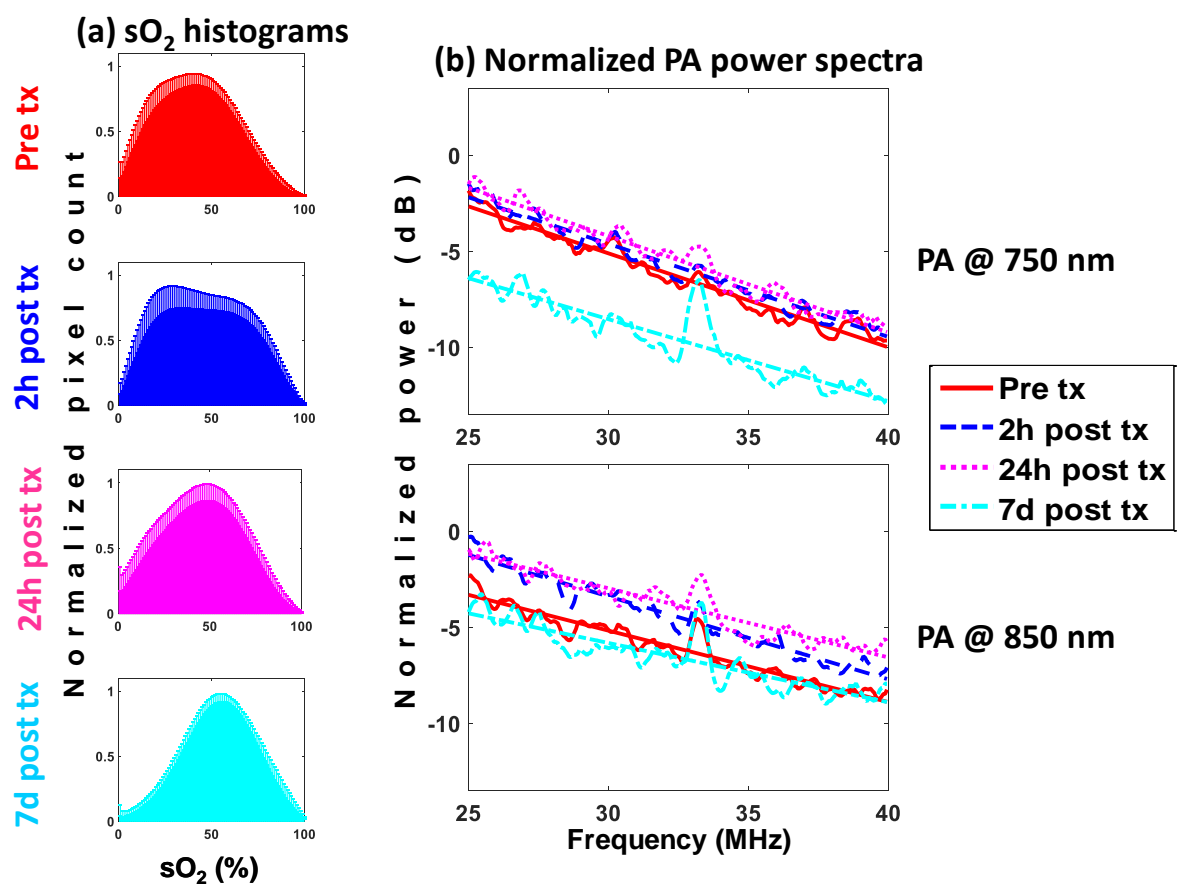

Figure 4-4: Saline-treated mice (a) tumor oxygenation histograms and the (b) normalized power spectra for PA images obtained at 750 (top) and $850 \mathrm{~nm}$ (bottom) for multiple imaging timepoints. The error bars on the histograms represent the standard deviation on the pixel count for each $\mathrm{sO} 2$ value obtained from 21 different histograms per timepoint per mouse. The power spectra are averages of at least $100 \mathrm{RF}$ lines per 21 tumor slices per timepoint ( 6 total) per mouse ( 7 total). All linear regressions had a Chi-squared value $\geq 0.9$. Treatment is abbreviated as $t x$. 


\section{CHAPTER 4}

\subsubsection{Correlation of QPA parameters with tumor oxygenation}

The $\mathrm{sO}_{2}$ mode was plotted against the PA spectral slope (SS) for HaT-DOX responders, the lone HaT-DOX non-responder and the Saline-treated mice (Figure 4-5). At each timepoint, the SS and $\mathrm{sO}_{2}$ mode is represented by the average value across all mice within a treatment group. A total of 21 planes were analyzed to examine the distribution of metrics across the entire tumor volume. The clustering of HaT-DOX responders by imaging timepoint was clear for both wavelengths (Figure 4-5a). The PA SS at 750 and $850 \mathrm{~nm}$ decreased by $45 \%$ and $73 \%$, respectively when the $\mathrm{sO}_{2}$ mode dropped at $30 \mathrm{~min}$ post-treatment. These changes in SS persist for the first $24 \mathrm{~h}$ posttreatment until the oxygenation of the tumors increased by $10 \%$ from $30 \mathrm{~min}$. During this interval, the SS further decreased by $8.5 \%$ and $11.5 \%$ for 750 and $850 \mathrm{~nm}$, respectively. By $7 \mathrm{~d}$ posttreatment, an increase in the average $\mathrm{sO}_{2}$ mode of $22 \%$ from pre-treatment was accompanied by a decrease of the SS (79\% for $750 \mathrm{~nm}$ and $113 \%$ for $850 \mathrm{~nm}$ ). PA estimates of tumor oxygen saturation reported here are also described in other reports. Mallidi and colleagues have used the VevoLAZR system to track the progression of photodynamic therapy and found tumor $\mathrm{sO}_{2}$ values to be around 40-60\% before and after the administration of vascular disrupting therapies [133]. In a more recent report, Rich and Seshandri reported good correlations between PA estimates of $\mathrm{sO}_{2}$ with oxygen-enhanced magnetic resonance imaging in head and neck xenograft tumors [136]. Additionally, $\mathrm{PA}$ estimates of $\mathrm{sO}_{2}$ reported here have also been previously correlated with hypoxia stains obtained from tumor sectioning and were found to adequately represent the tumor microenvironment [268]. 


\section{CHAPTER 4}

(a)

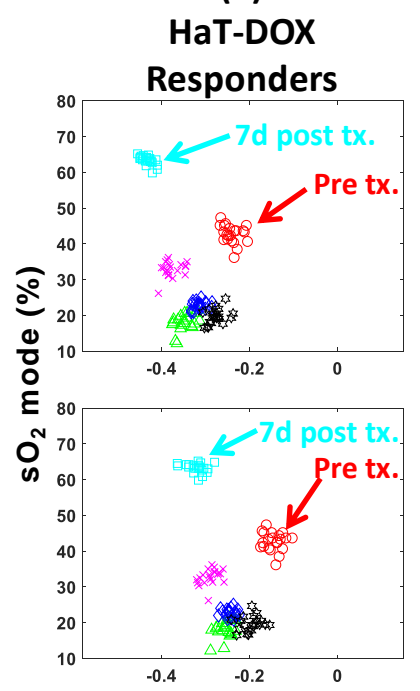

(b)

HaT-DOX Non-Responder
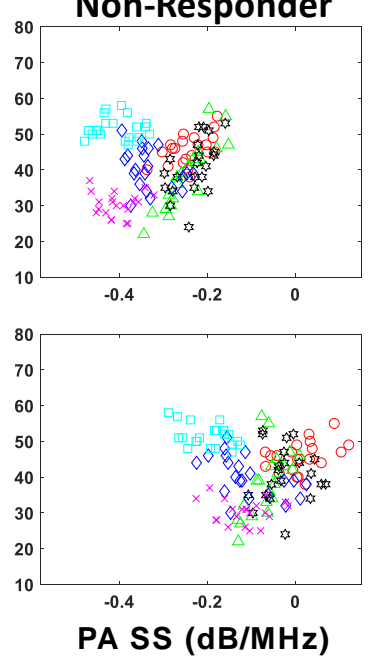

(c)

Saline

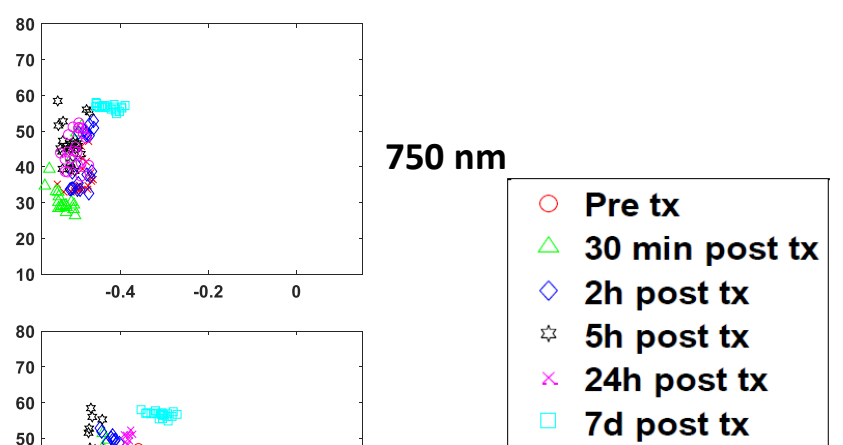

$850 \mathrm{~nm}$

Figure 4-5: Oxygen saturation ( $\mathrm{sO} 2$ ) mode versus the PA spectral slope (PA SS). The PA SS is computed for $750 \mathrm{~nm}$ (top row) and $850 \mathrm{~nm}$ (bottom row) and for HaT-DOX (a) responders $(\mathrm{n}=$ $5)$ and (b) non-responder $(n=1)$ and (c) Saline $(n=7)$. Each dot represents the average SS across at least 100 PSnorm within 21 tumor slices per timepoint, per mouse.

The HaT-DOX non-responder (Figure 4-5b) does not exhibit the same behavior as the HaT-DOX responders. Vessel oxygenation did not significantly drop 24 hours post-treatment and a higher variation in the distribution of SS values was observed throughout the imaging timepoints. Furthermore, there were no identifiable trends in the SS during treatment other than a $0.2 \mathrm{~dB} / \mathrm{MHz}$ difference in the PA SS for $850 \mathrm{~nm}$ compared to $750 \mathrm{~nm}$ observed at all imaging timepoints for this mouse.

The Saline SS exhibited a distinctive behavior, differentiating them from the HaT-DOX mice (Figure 4-5c). The $750 \mathrm{~nm}$ SS did not significantly change at the early timepoints as shown by the clustering around $-0.48 \mathrm{~dB} / \mathrm{MHz}$. This was also correlated with the lack of $\mathrm{sO}_{2}$ changes at early timepoints. The $850 \mathrm{~nm} \mathrm{SS}$ increased by $14 \%$ from pre-treatment when the $\mathrm{sO}_{2}$ increased $20 \%$ at the $7 \mathrm{~d}$ timepoint. For the $850 \mathrm{~nm}$ illumination, the SS increased slightly (3\%) at the $24 \mathrm{~h}$ timepoint before increasing by $16 \%$ at $7 \mathrm{~d}$ post-treatment. Overall, the $850 \mathrm{~nm} \mathrm{SS}$ is $\sim 0.1 \mathrm{~dB} / \mathrm{MHz}$ higher than $750 \mathrm{~nm}$ and lower than the HaT-DOX SS. The significance of these findings can be established by examining the correlation between the SS and the optical absorber (i.e. vascular morphology) [186], [187]. The lack of large changes in the SS for the Saline and HaT-DOX non- 


\section{CHAPTER 4}

responder implies that the tumor vasculature is responding differently to treatments. It is worth noting that the differences in the SS trends post-treatment are sufficient to identify the nonresponder and the control group.

\subsubsection{Histological examinations post-treatment}

Representative cross-sections of tumors extracted after the $2 \mathrm{~h}$ imaging timepoint are shown inFigure 4-6. A large amount of FITC-lectin leakage was observed in the of vicinity blood vessels (Figure 4-6a). Overall, 70\% of all vessels within the HaT-DOX-treated tumor were FITC-lectin positive compared to just $30 \%$ for the Saline treatment at $2 \mathrm{~h}$ (Figure 4-6b). Figure 4-6c shows the distribution of vessel size within the tumor measured $2 \mathrm{~h}$ post-treatment. The Saline group had a larger number of intact vessels post-treatment compared to the HaT-DOX group, except for vessels smaller than $25 \mu \mathrm{m}$.

The mechanism of action for treatments like HaT-DOX is the rapid ( $<20$ seconds), burst-release of DOX upon exposure to mild-hyperthermia [235], [269]. The formation of large DOX concentration gradients from the tumor vasculature to the tumor interstitial space leads to DOXinduced endothelial cell damage. The damaged endothelial cells become unable to support the circulating blood and its contents, eventually succumbing to hemorrhage, blood coagulation, pooling and localized vascular shutdown [212], [266]. This phenomenon was observed in Figs. 6a and $6 \mathrm{~b}$ for tumors treated with HaT-DOX.

The recently published study by our group examined the correlation between $\mathrm{sO}_{2}$ and vascular damage due to a single dose of HaT-DOX released during mild-hyperthermia [150]. At the 2 hour timepoint, the drop in $\mathrm{sO}_{2}$ was correlated with an increase in FITC-lectin leakage within the tumor interstitium. At 750/850 nm, the most dominant optical absorber is RBC' hemoglobin [223]. Given the significant amount of FITC in the vicinity of post-treatment blood vessels (Fig. 6a), treatment leads to an increased number of leaked RBCs in the interstitium, where they are no longer capable of circulating and unable to exchange pulmonary oxygen, and so eventually become deoxygenated. We hypothesize that this contributes to the post-treatment decrease in tumor oxygenation (Figure 4-3a). Furthermore, the hemorrhage of RBCs in the intersitium of tumors inadvertently forms an optical target with the same absorption properties as RBCs but larger effective size (i.e. RBCs are 


\section{CHAPTER 4}

no longer confined to the blood vessels). The formation of this "pool" of RBCs outside the vessels may be responsible for the changes in the spectral slope shown in Figure 4-5a. Previous experimental studies by our group and others have demonstrated that an increase in the effective optical absorber size causes a decrease in the PA SS [144], [176], [187], [251], [267]. Moreover, this trend was also confirmed in a simulation study which examined the effect of vascular hemorrhaging on the QPA SS parameter [270]. By 7 days post-treatment, both groups had sufficient time to recruit and generate new blood vessels. The effects of re-vascularization following cancer treatments have been previously studied using other modalities [26], [43], [74] and are consistent with the observed increase in tumor $\mathrm{sO}_{2}$ reported in the current study at 7 days.

The post-treatment changes in the PA SS at both wavelengths are expected to be dependent on the size of vessels inside the tumor post-treatment as compared to pre-treatment. Changes in the distribution of tumor vessels are shown in Figure 4-6c. This is consistent with the experimental results: the PA SS of the Saline group was more negative than for the HaT-DOX group (Figure 4-5c). Additionally, the sub-resolution sized vessels were found to be more abundant in the HaTDOX treated group. Although smaller than the system spatial resolution (axial $45 \mu \mathrm{m}$, lateral 90 $\mu \mathrm{m})$ the PA signal from collections of these vessels can contribute to the recorded RF PA signal and the SS. This is a phenomenon that is observed during studies of acoustic-resolution PA imaging where speckle dominates the images due to collections of unresolved sources of PA waves [176], [249], [271]. 


\section{CHAPTER 4}
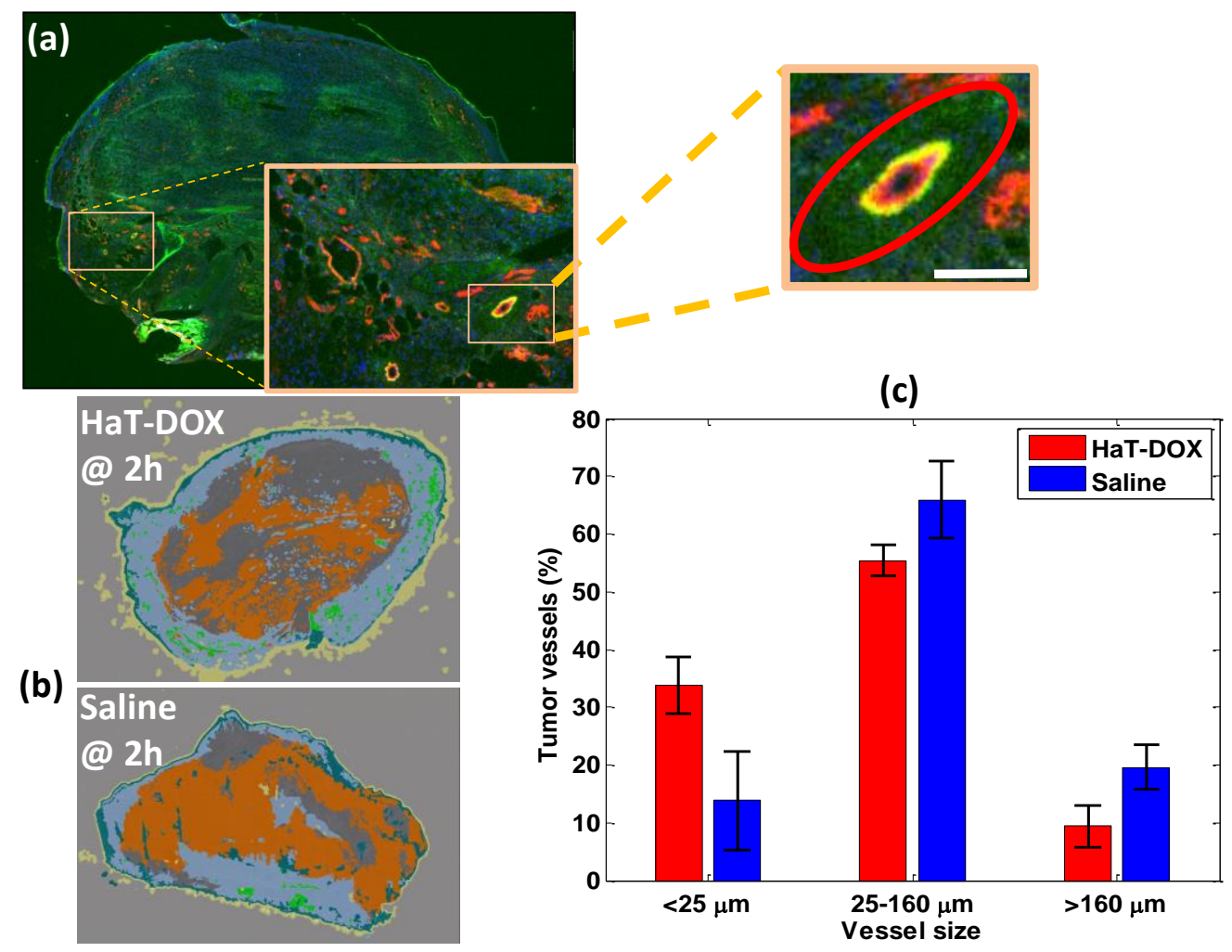

Figure 4-6: Tumor histology obtained $2 \mathrm{~h}$ post-treatment. (a) Representative images of HaT-DOXtreated tumor blood vessels ( $2 \mathrm{~h}$ post-treatment) stained with CD-31 (red and yellow) showing the leakage of FITC-lectin (green) in their vicinity (red ellipse). The width of the image is $10 \mathrm{~mm}$ and the scale-bar in the zoomed inset is $100 \mathrm{~mm}$. (b) Representative HaT-DOX and Saline tumors showing the proportion of FITC-lectin leakage (dark gray) outside of the tumor area (orange). Normal tissue is the teal color surrounding the tumor and the slide background is light gray. For more details on how these images were obtained refer to reference [43]. (c) Distribution of the size of tumor blood vessels for HaT-DOX and Saline measured at the $2 \mathrm{~h}$ timepoint.

\subsubsection{Quantitative ultrasound for detecting tumor cell death}

In order to compare the treatment monitoring capabilities of QPA with quantitative ultrasound (QUS), the SS from the US imaging of the tumors was computed for all the imaging timepoints and treatments (Figure 4-7). It is evident that QUS is unable to identify changes in the structure of the tumor as early as a few hours post-treatment in the same manner as the PA SS. The US SS does not significantly change in HaT-DOX treated tumors until the 7d timepoint despite the large drop in oxygenation (Figure 4-7a). At 7d, the US increased by nearly 35\%, which is indicative of a decrease in the size of the scatterers (i.e. tumor cells seen) due to the structural changes that the tumor has undergone. No noticeable trend was observed in the SS of the HaT-DOX non-responder (Figure 4-7b). The SS of the Saline control (Figure 4-7c) increased by $24 \%$ at $7 \mathrm{~d}$ compared to pre- 


\section{CHAPTER 4}

treatment. It is important to note that by $7 \mathrm{~d}$ there were no significant changes in the size of the tumors between all groups, as assessed through caliper measurements (Figure 4-2).

(a)

HaT-DoX

Responders

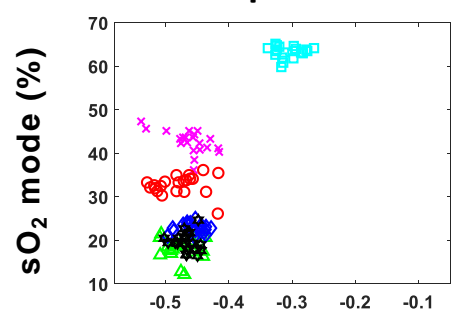

(b)

HaT-DOX Non-Responder

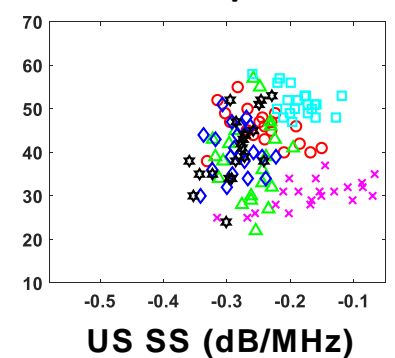

(c) Saline

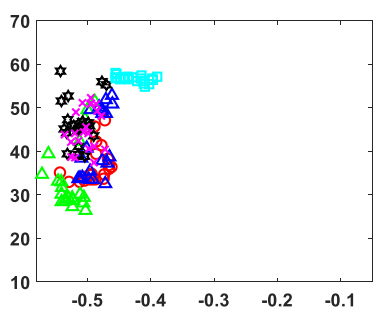

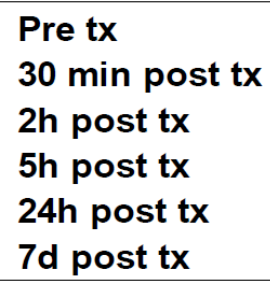

Figure 4-7: The correlation between US SS and sO2 mode for HaT-DOX (a) responders and (b) non-responder and (c) Saline. Each dot represents the average SS across at least 100 RF spectra within 21 tumor slices per timepoint per mouse.

The examination of the changes that the tumor cells undergo during and after treatment is another aspect of treatment monitoring. QUS has been successful in probing the structural changes that occur within tumor cells when they are exposed to changing environmental conditions [33], [34], [272]. Recently, the technique was used to evaluate the treatment response of breast and prostate cancer patients before conventional imaging modalities [24], [35], [232]. However, the technique is sensitive to localized changes in the structure of the tumor cells and cannot provide functional information on the tumor vasculature in the same manner as PA imaging. Additional complexity arises from the fact that inflammation of the tumors was present one week after treatment. This could complicate interpretation of results, as changes in the acoustic properties of the tumor microenvironment have been shown to affect QUS parameters such as the SS [273]. This renders the interpretation of changes in parameters more challenging than in QPA where the specific, oxygen dependent optical absorption of hemoglobin is known to dominate the PA signal generation. Therefore, there is a more direct link to the relation between the changes detected in the PA signals collected and how these can be interpreted based on the underlying biophysical changes in tissue structure that occur because of the treatment. The latter is an important consideration in using QPA for monitoring vascular-targeted therapies. 


\section{CHAPTER 4}

\subsection{Conclusions}

Our study demonstrates the potential of using PA imaging and QPA for monitoring treatmentinduced changes in the tumor vasculature. Changes in quantitative photoacoustics as early as 30 minutes post-treatment are strongly correlated with a significant drop in oxygenation observed at the same timepoints. These correlated values continue through to 5 hours where they are shown to correlate with changes to the vasculature as observed via histology. In fact, the spectral analysis

of the PA RF data provides evidence for the loss of oxygenation due to HaT-DOX-induced vascular destruction. Our results indicate that the frequency content of the PA data provides useful information related to the changes in the morphology of blood vessels during treatment. Comparisons with QUS suggest that the QPA technique is capable of identifying treatment responders as early as a few hours post-treatment for treatments that disrupt the vasculature. This study establishes the feasibility of using PA imaging and spectroscopy for treatment monitoring and should be used to assist with the translation of PA imaging to mainstream radiology.

\subsection{Acknowledgements}

Funding for this project was provided in part by the generous financial support of the Terry Fox Foundation, the Natural Sciences and Engineering Research Council of Canada (NSERC), the Canada Research Chairs Program, the Ontario Institute for Cancer Research and the Canadian Institutes for Health Research (CIHR). EH is supported by an NSERC Vanier Canada Graduate Scholarship. SDL is supported by the Prostate Cancer Foundation and CIHR Young Investigator Awards We would like also to acknowledge the technical support received by the STTARR facility at the University Health Network and Fujifilm-Visual Sonics. 


\section{Photoacoustic imaging biomarkers for monitoring biophysical changes during nanobubble-mediated radiation treatment}

\section{The contents of this chapter have submitted for publication in Photoacoustics.}

E Hysi, MN Fadhel*, Y Wang*, JA Sebastian, A Giles, GJ Czarnota, AE Exner and MC Kolios. "Photoacoustic imaging biomarkers for monitoring biophysical changes during nanobubblemediated radiation treatment" Photoacoustics 2020; under review.

*The authors contributed equally to this work.

\subsection{Introduction}

Despite the standardization of treatments such as surgery, chemotherapy and radiation therapy, overall cancer survival rates remain modest [274], [275]. A deeper understanding of cancer causation and progression has driven the development of molecularly targeted drugs. These have led to personalized, precision treatments that rely on individualized drug administration based on prognostic and predictive markers of response [7]. The ultimate hope is that such a personalized strategy in cancer therapy will replace the "one-size-fits-all" conventional approach, increasing overall survival rates [8]. This however remains a monumental task because the selection of the right treatment is a complex decision based on continuously changing molecular diagnostics and rapidly evolving biomedical literature [276].

Equally important to the developments of new drugs or experimental therapies is their assessment with inexpensive and readily available means, which can expedite their clinical translation [277]. A prime example of a successful biomarker-driven personalized cancer therapy is the development of trastuzumab for human epidermal growth factor 2 (HER2)-positive breast cancer [278]. However, the molecular heterogeneity of tumors can also lead to individualized and dynamic treatment-induced changes in tumor morphology and structure [9]. Unfortunately, current treatment efficacy assessment approaches might not be suitable for handling the complexities of personalized cancer treatments [9]. Studying the structural and functional markers of treatment response is thus imperative for providing fast and reliable feedback on treatment efficacy [276]. 


\section{CHAPTER 5}

Photoacoustic (PA) imaging is a relatively new modality that combines ultrasound (US) resolution with optical contrast. The optical absorption of pulsed laser light generates mechanical sound waves from the thermoelastic expansion of endogenous tissue chromophores [279]. In PA imaging, the strength of the signal depends on the tissue chromophore identity and concentration. As such, PA imaging has contributed to breast cancer diagnosis, mapping neuronal functional connectivity, quantifying pharmacokinetics of novel drugs and detecting the presence of malaria [101], [108], [148], [248].

While a lot of strides have been made in the diagnostic realm [104], [106], [247], [280], PA imaging applications in cancer treatment monitoring are still in their infancy. A handful of preclinical studies have illustrated the potential of PA imaging for the early monitoring of cancer therapies by mapping the tumor oxygenation changes post-treatment through measurements of oxygen saturation [133], [136], [150], [151], [258]. The clinical translation of PA imaging in cancer treatment monitoring warrants the development of non-invasive biomarkers whose detection and quantitative measurement is accurate, reproducible and feasible over time [7]. Just like for the US radiofrequency (RF) signals in quantitative ultrasound spectroscopy [29], [30], the frequencies of the PA signals carry information on the structure (size, shape and orientation) of the underlying absorber [124], [129], [176], [281]. In utilizing the frequency information of the PA RF signals, acoustic resolution PA imaging has the potential to probe non-resolvable optical absorbers and thus provide new PA biomarkers that can be linked to the success or failure of the therapy being evaluated.

In this study, we investigate for the first time the therapeutic application of ultrasonically activated nanobubbles (NBs) for enhancing radiation therapy. Ultra-stable NBs (100-300 nm diameter) are a new class of US contrast agents with contrast-enhancing capabilities comparable to conventional microbubbles (MBs, 1-10 $\mu \mathrm{m}$ diameter) [282]-[285]. The size range makes MBs ideal vascular disrupting agents. Under US stimulation, they can cause increased vascular permeability for amplifying tumoral drug release [286], inducing thrombolysis of blood clots [287] and open the blood brain barrier [288]. In contrast, NBs have been shown to remain echogenic at the clinical frequency range (3-12 MHz) [289] while being able to exit tumor vasculature due to the enhanced permeability and retention effect [66]. Unlike conventional MBs that remain intravascular, NBs 


\section{CHAPTER 5}

can in principle directly target tumoral cells, thus having the potential to become multifunctional theranostic agents. The prolonged in vivo stability has allowed NBs to be used to open the blood brain barrier [282], enhance contrast in ovarian cancer [290] and increase chemotherapy uptake [283].

The ultrasonic stimulation of conventional MBs has been shown to enhance radiation therapy [291]-[293]. This combination treatment synergistically damages the endothelial lining of tumor blood vessels in addition to causing radiation-induced damage of cancer cells in vivo. However, a limitation of any MB-based treatments remains their limited in vivo stability [294] which reduces their circulation time and usability [285]. NB-enhancement of radiation therapy has the potential to further maximize the tumoral cell death through their extravascular effect. Histopathological examinations post-treatment have provided insights into the biophysical mechanism of MB treatments [293]. The damage caused to the vascular endothelium through mechanical perturbations and the production of reactive oxygen species can lead to secondary tumor cell death and vascular collapse. Here we investigate whether PA imaging biomarkers can quantify the vascular damage induced by ultrasound excitations of NBs combined with radiation, and if these biomarkers can be used to monitor treatment efficacy.

\subsection{Materials and methods}

\subsubsection{Animal model and tumor growth}

Severe combined immuno-deficient (SCID) male mice (Charles River Laboratories International, Wilmington, MA, USA) were inoculated with $10^{6}$ human prostate cancer cells (PC3, American Type Culture Collection, Manassas, VA, USA) in order to grow xenograft tumors in the right, upper hind leg of each mouse $(\mathrm{n}=58)$. Tumors were grown to 8 to $10 \mathrm{~mm}$ in maximum diameter for approximately 4-6 weeks. Following treatments, all animals were euthanized by cervical dislocation under anesthesia after 24 hours in order to histologically assess the morphological changes to the tumor cells and blood vessels. The animal studies were approved by the Sunnybrook Research Institute Animal Ethics Committee and conformed to the Canadian Council on Animal Care guidelines. 


\section{CHAPTER 5}

\subsubsection{Treatment groups}

A total of 58 mice were divided into six treatment groups as summarized in Table 5-1.

Table 5-1: Experimental treatment groups for the MB and NB experiments.

\begin{tabular}{l||c}
\multicolumn{1}{c||}{ Treatment group (abbreviation) } & Number of animals \\
\hline \hline Untreated control (0Gy) & 14 \\
\hline Radiation only, 8 Gy, single fraction (8Gy) & 14 \\
\hline Microbubbles + Ultrasound (MB+US) & 8 \\
\hline Nanobubbles + Ultrasound (NB+US) & 5 \\
\hline Microbubbles + Ultrasound + Radiation (NB+US+8Gy) & 12 \\
\hline Nanobubbles + Ultrasound + Radiation (NB+US+8Gy) & 5 \\
\hline
\end{tabular}

\subsubsection{Ultrasound-activated microbubble and nanobubble treatments}

\subsubsection{Microbubble preparations and injections}

Commercially available Definity (Lantheus Medical Imaging, N. Billerica, MA, US) MBs ( 3 $\mu$ m mean diameter) were activated using the Lantheus Vialmix mechanical shaker device (45 seconds at $3000 \mathrm{rpm})$. An intravenous tail vein injection of a MB bolus $\left(1.2 \times 10^{10} \mathrm{MBs} / \mathrm{ml}\right)$ was administered to each mouse 5 minutes prior to the ultrasound activation.

\subsubsection{Nanobubble preparations and injections}

NBs were prepared using a previously established protocol [285]. The NBs consisted of a lipid shell and an octafluoropropane $\left(\mathrm{C}_{3} \mathrm{~F}_{8}\right.$, Electronic Fluorocarbons LLC, Hatfield, PA, USA) gas. The NB consisted of the following mixture of lipids (Avanti Polar Lipids, Inc.):

- 1,2-dipalmitoyl-sn-glycero-3-phosphoethanolamine

- 1,2-dibehenoyl-sn-glycero-3-phosphocholine

- 1,2-distearoyl-sn-glycero-3-phosphoethanolamine-N-[methoxy (polyethylene glycol)-2000]

- 1,2-dipalmitoyl-sn-glycero-3-phosphate

The lipid mixture with a 2:6:1:2 ratio was dissolved in propylene glycol and glycerol in Phosphate Buffered Saline (PBS) solution. The air present in the lipid-PBS solution was replaced by $\mathrm{C}_{3} \mathrm{~F}_{8}$ prior to mechanically agitating the lipid-gas solution for 45 seconds using the Lantheus Vialmix, generating a mixture of MBs and NBs. In order to isolate the NBs from the mixture foam and the MB solution, centrifugation was performed at $50 \mathrm{~g}$ for 5 minutes. The NB solution was retrieved 


\section{CHAPTER 5}

with the headspace vial inverted and by using a $21 \mathrm{G}$ needle withdrawing from a fixed distance of $5 \mathrm{~mm}$ from the bottom of the vial.

The size and concentration of the resulting NB solution were assessed using resonant mass measurement (Archimedes ${ }^{\circledR}$, Malvern Panalytical, Westborough, MA, USA) [284]. This device was equipped with a nanosensor which can measure particle sizes between 50 and $2000 \mathrm{~nm}$. The NB solution was diluted by a factor of $1000(\mathrm{v} / \mathrm{v})$ in PBS prior to measurements. A size distribution representative of the NB formulation used in this study is shown in Supplementary figure 5.A. The average diameter of the NB was measured to be $205 \pm 97 \mathrm{~nm}$. NBs were injected 5 minutes prior to ultrasound activation with a tail vain injection concentration of $1 \times 10^{11} \mathrm{NBs} / \mathrm{ml}$.

\subsubsection{Low frequency ultrasound activation}

Following the MB or NB bolus injections, $500 \mathrm{kHz}$ frequency US treatments were administered 5 minutes post-injection. Figure 5-1 shows a schematic of the treatment and imaging setups used to expose the MB and NB post-injection. The mice were secured onto a mounting platform with the lower half of the mouse and the tumor immersed in a degassed water bath kept at $37^{\circ} \mathrm{C}$ (Figure 5-1a). The platform was mounted coaxially to a therapeutic ultrasonic transducer such that the tumor was centered within the focus of the transducer through a micro-positioning system.

The low frequency ultrasonic activation of the MBs and NBs was generated using a $500 \mathrm{kHz}$ focused transducer (Valpey Fisher Inc., Hopkinton, MA, USA) [291]-[293]. The transducer had a diameter of $28.7 \mathrm{~mm}$, a focal depth of $85 \mathrm{~mm}$, peak negative pressure of $570 \mathrm{kPa}$, a $-6 \mathrm{~dB}$ beam width of $31 \mathrm{~mm}$ and a depth of field greater than $20 \mathrm{~mm}$. It was connected to a PC-controlled digital acquisition system (Agilent Technologies Inc., Monroe, NY, USA) which controls a waveform generator (Tektronix Inc., Beaverton, OR, USA), a power amplifier and receiver (Ritec Inc., Warwick, RI, USA) using the setup previously described [295].

Following tail vein injection, bubbles were allowed to circulate for 5 minutes before ultrasonic activation. The ultrasonic activation treatment was administered for a total time of 5 minutes using 16 cycle tone bursts of $500 \mathrm{kHz}$ frequency with a pulse repetition frequency of $3 \mathrm{kHz}$. This resulted in a duty cycle of $0.24 \%$ or $720 \mathrm{~ms}$ of beam-on treatment time. This low duty cycle was designed 


\section{CHAPTER 5}

to avoid any bioeffects potentially rising from ultrasound heating. It was previously estimated that for this system the transducer spatial peak temporal peak intensity was $22.4 \mathrm{~W} / \mathrm{cm}^{2}$ and the spatial peak temporal average intensity was $0.054 \mathrm{~W} / \mathrm{cm}^{2}$ [295]. The $-3 \mathrm{~dB}$ beam width was determined to be $15.6 \mathrm{~mm}$, ensuring that the entire tumor volume (diameter 8-10 $\mathrm{mm}$ ) is consistently exposed to the ultrasound pulse.

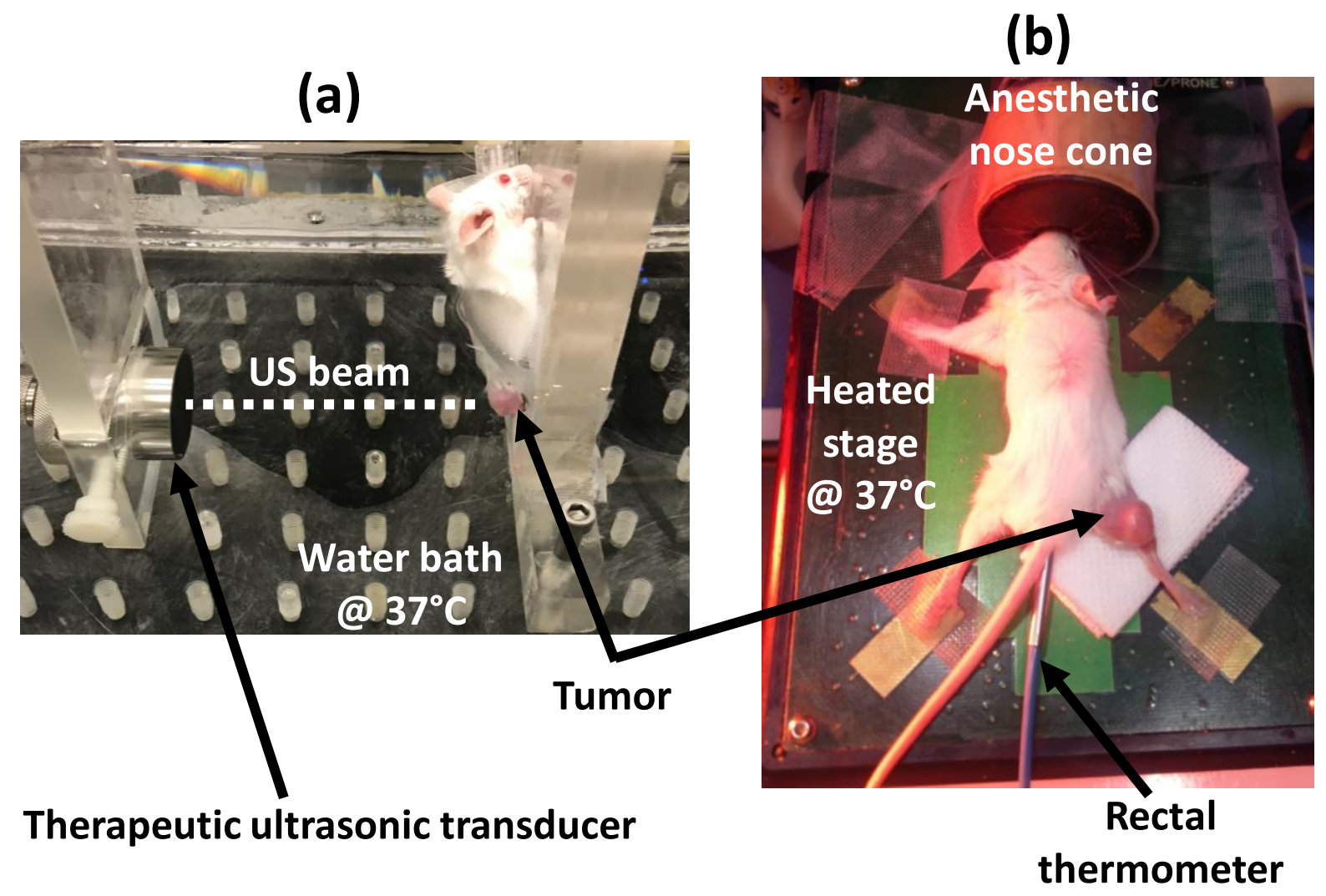

Figure 5-1: Ultrasonic treatment and PA imaging setups. (a) Ultrasonic bubble activation setup. The tumor is positioned at the focus of the therapeutic ultrasonic transducer. (b) PA imaging platform illustrating the prone imaging position of the mouse during imaging. The mice are kept at physiological temperature through a heated stage and a heating lamp.

\subsubsection{Radiation treatments}

Following the $\mathrm{MB}$ or $\mathrm{NB}$ treatments, a single dose of $8 \mathrm{~Gy}$ X-ray radiation was delivered through a $160 \mathrm{kVp}$ small animal irradiator (Faxitron Bioptics, LLC, Tucson, AZ, USA). The dose was delivered at a rate of $200 \mathrm{cGy} / \mathrm{min}$ (duration $=4$ minutes), three hours following ultrasound treatments to maximize the degree of tumor cellular death [293]. The mouse was shielded with a lead sheet and a circular cutout in the hind leg enabled the irradiation of only the tumor. 


\section{CHAPTER 5}

\subsubsection{Delayed tumor growth investigations}

Another cohort of mice $(n=19)$ bearing subcutaneous PC3 tumors were treated either with a single dose of 8 Gy $(n=5), N B+U S(n=5), N B+U S+8 G y(n=4)$ or were left untreated $(n=5)$. Instead of sacrificing at the 24-hour time point, the mice were followed longitudinally post-treatment for monitoring the growth delay effects of each treatment. The tumor size was measured via standard caliper measurements every 3-5 days for 30 days post-treatment.

\subsubsection{Photoacoustic imaging and biomarkers}

\subsubsection{Imaging setup}

Figure 5-1b shows the positioning setup for performing PA imaging on the hind leg PC3 tumors of SCID mice. The VevoLAZR small animal PA imaging system (Fujifilm-VisualSonics, Toronto, Canada) was used to perform three-dimensional (3D) volume of the tumors. A $21 \mathrm{MHz}$ linear array probe with 256 active transducer elements (LZ 250) was coupled to an Nd:YAG laser operating through an optical parametric oscillator in the 680-970 $\mathrm{nm}$ range [175]. The laser light (30 $\mathrm{mJ} /$ pulse, $20 \mathrm{~Hz}$ pulse repetition frequency, $6 \mathrm{~ns}$ pulse length) was delivered through a pair of optical fibers terminating in two rectangular strips located at a $30^{\circ}$ angle on both sides of the acoustic aperture. Co-registered US and PA images were acquired at a rate of 5 frames/second at $750 \mathrm{~nm}$ and $850 \mathrm{~nm}$. Each mouse was anesthetized using isoflurane (1.5\%) to immobilize them during imaging (Figure 5-1b). The mice were imaged in the prone position in order to fully expose their hind leg tumor. Their core temperature was kept physiological with the help of an external heating lamp and a heated platform. The center of the tumor was positioned at the focus of the US/PA transducer $(11 \mathrm{~mm})$ and clear ultrasonic gel was used at provide acoustic coupling. The probe was scanned from the proximal towards the distal end of the tumor in $80 \mu \mathrm{m}$ steps. At every step, a two-dimensional (2D), co-registered US and PA image was acquired for both imaging wavelengths at pre-treatment, 2 hours, and 24 hours post-treatment.

\subsubsection{Vessel oxygenation biomarkers}

Figure 5-2 shows a schematic overview of the PA biomarkers that were used in this work to examine the impact that ultrasound-simulated $\mathrm{MB}$ and $\mathrm{NB}$ treatments in combination with radiation have on the tumor viability and its blood vessels. Upon the administration of the MB or 


\section{CHAPTER 5}

$\mathrm{NB} /$ radiation treatments, two-wavelength $\left(\lambda_{1}=750 \mathrm{~nm}\right.$ and $\left.\lambda_{2}=850 \mathrm{~nm}\right)$ PA images were acquired at each scanning location in the tumor. The oxygen saturation $\left(\mathrm{sO}_{2}\right)$ and the total hemoglobin $(\mathrm{HbT})$ content present within the vessels were calculated by assuming that deoxy $(\mathrm{Hb})$ and oxy (HbO) hemoglobin are the dominant absorbers inside tumor blood vessels [223]. At every 2D tumor slice, the $\mathrm{sO}_{2}$ and $\mathrm{HbT}$ are calculated by:

$$
\begin{aligned}
& s O_{2}=\frac{[H b O]}{[H b O]+[H b]}=\frac{\mu_{a}^{\lambda_{2}} \varepsilon_{H b}^{\lambda_{1}}-\mu_{a}^{\lambda_{1}} \varepsilon_{H b}^{\lambda_{2}}}{\mu_{a}^{\lambda_{1}} \Delta \varepsilon_{H b}^{\lambda_{2}}-\mu_{a}^{\lambda_{2}} \Delta \varepsilon_{H b}^{\lambda_{1}}} \\
& H b T=[H b O]+[H b]=\frac{\mu_{a}^{\lambda_{1}} \Delta \varepsilon_{H b}^{\lambda_{2}}-\mu_{a}^{\lambda_{2}} \Delta \varepsilon_{H b}^{\lambda_{1}}}{\varepsilon_{H b}^{\lambda_{1}} \varepsilon_{H b O}^{\lambda_{2}}-\varepsilon_{H b}^{\lambda_{2}} \varepsilon_{H b O}^{\lambda_{1}}}
\end{aligned}
$$

where, $\mu_{a}$ is the absorption coefficient in units of $\mathrm{cm}^{-1} ; \varepsilon_{H b O}$ and $\varepsilon_{H b}$ are the known molar extinction coefficients (in units of $\mathrm{cm}^{-1} \mathrm{M}^{-1}$ ) of $\mathrm{HbO}$ and $\mathrm{Hb}$, respectively; $\Delta \varepsilon_{H b}=\varepsilon_{H b O}-\varepsilon_{H b}$; and $[\mathrm{HbO}]$ and $[\mathrm{Hb}]$ are the concentrations (in units of $\mathrm{M}$ ) of the two forms of hemoglobin, respectively. The optical absorption coefficients were estimated by the PA signal amplitude [190], [296]. The latter was estimated by calculating the envelope of the time-domain PA signal within the tumor region of interest. Using a histogram-based quantification approach developed by our group [150], [151], the $\mathrm{sO}_{2}$ and $\mathrm{HbT}$ values of each 2D slice within the tumor was calculated from the mode of the $\mathrm{sO}_{2}$ and $\mathrm{HbT}$ histograms, respectively. At every imaging timepoint, the average mode was computed throughout the entire tumor volume for every mouse and then averaged across all animals belonging to the same treatment group to examine inter-treatment variation in tumor oxygen saturation and total hemoglobin level. Additionally, the distribution of the $\mathrm{sO}_{2}$ and $\mathrm{HbT}$ across the tumor volume was also examined by plotting each $\mathrm{sO}_{2}$ or $\mathrm{HbT}$ mode value from $2 \mathrm{D}$ images as a single datapoint across multiple treatments/timepoints. Lastly, the differences relative to the pre-treatment timepoint for each mouse was calculated and the percent change from the control mice were computed. 


\section{CHAPTER 5}

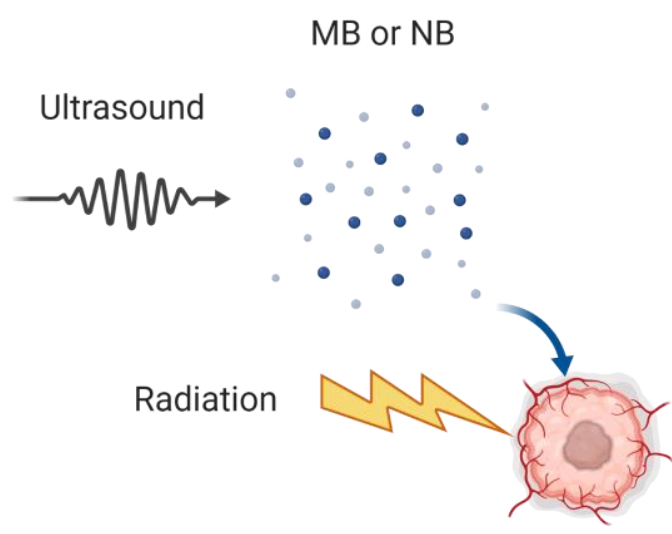

Tumor
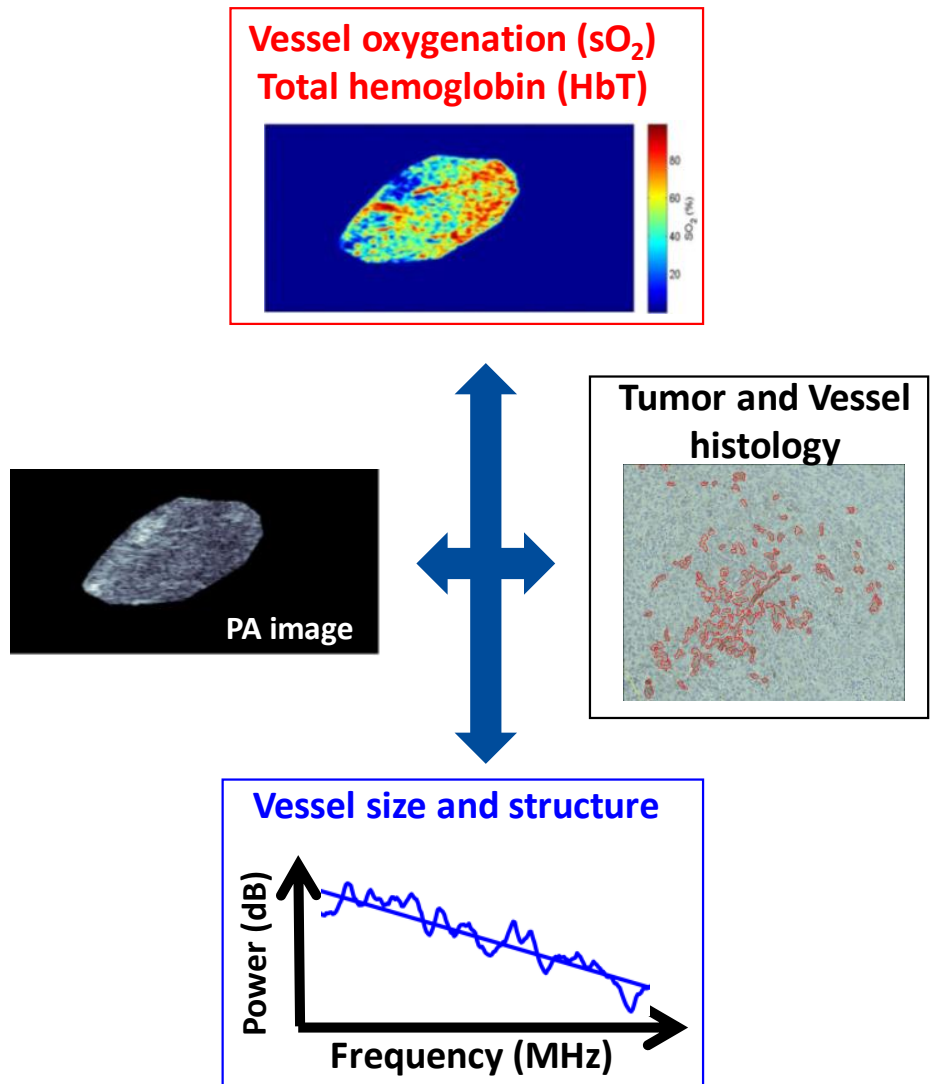

Figure 5-2: PA imaging biomarkers of treatment response. The effects of the combination of ultrasound-simulated $\mathrm{MB}$ or NB treatments with radiation can be probed using PA imaging biomarkers (functional and structural) and can be correlated with histological metrics of tumor cells and blood vessels. Treatment and tumor illustrations were created with Biorender.com. Abbreviations: $\mathrm{MB}=$ microbubble; $\mathrm{NB}=$ nanobubble; $\mathrm{sO} 2=$ oxygen saturation $; \mathrm{HbT}=$ total hemoglobin.

\subsubsection{Vessel size and structure biomarkers}

The time/spatial domain PA radiofrequency (RF) signals acquired at both wavelengths of illumination $p(x, y, z, \lambda)$ were used to compute the normalized PA power spectrum as a function of acoustic frequency $f$ [151], [297]. The wavelength-dependent, normalized power spectrum at every $z^{\text {th }} 2 \mathrm{D}$ scan of the tumor $P S_{\text {norm }}(f, z, \lambda)$ was calculated by:

$$
P S_{\text {norm }}(f, z, \lambda)=\frac{1}{S} \sum_{i=1}^{S} \log _{10}\left[\frac{P S\left(f, z_{i}, \lambda\right)_{\text {tumor }}}{P S\left(f, z_{i}, \lambda\right) \text { ref }}\right]^{2}
$$




\section{CHAPTER 5}

where, $P S(f, \mathrm{z}, \lambda)_{\text {tumor }}$ and $P S\left(f, z_{i}, \lambda\right)_{\text {ref }}$ are the power spectra for the tumor and reference phantom, respectively. They were computed by taking the Fast Fourier Transform of all the RF

lines comprising each 2D scan within the tumor and the reference phantom. The latter was a gelatin-based construction of black carbon spheres (diameter 1-12 $\mu \mathrm{m}$ ) [176] which was imaged at the end of each timepoint using the same imaging settings as the tumors to remove the system dependencies. The normalized power spectra were fitted to a straight line as shown in Figure 5-2 and the PA spectral slope (SS) was extracted from:

$$
P S_{f i t}(f)=S S \times f+Y_{\text {int }}
$$

where, $P S_{f i t}(f)$ is the linear fit obtained from performing linear regression on the $P S_{\text {norm }}(f, z, \lambda)$ in the 10-30 MHz - $6 \mathrm{~dB}$ range of the linear array probe and $Y_{\text {int }}$ is the y-intercept of the fit measured in $\mathrm{dB}$. As was done with the $\mathrm{sO}_{2}$ and $\mathrm{HbT}$ parameters, the average $\mathrm{SS}$ value was computed for every $2 \mathrm{D}$ scan within the tumor in addition to the percent change from the pre-treatment values and untreated control group. This was done for both wavelengths of illumination 750 and $850 \mathrm{~nm}$ to examine the oxygen-dependent trends in SS.

\subsubsection{Histological analyses and correlations with PA imaging}

All animals were sacrificed after the 24 hours post-treatment imaging timepoint. The excised tumors were fixed in 10\% paraformaldehyde (volume/volume in PBS) overnight and were subsequently embedded in paraffin. Representative sections were taken from the approximate center of each tumor and stained with standard hematoxylin and eosin (H\&E). The vasculature of tumors was stained through endothelial cluster of differentiation 31 (CD31) staining (Figure 5-3a - Figure 5-3c). The degree of apoptotic cell death was assessed by deoxynucleotidyltransferasemediated-dUNP-biotin nick end labeling (TUNEL) staining (Figure 5-3d and Figure 5-3e). H\&E, TUNEL and CD31 sections were sequentially acquired within $10 \mu \mathrm{m}$ of each other.

The slides were digitized using the ZEISS Axio Scan.Z1 (Carl Zeiss Canada, Ltd., Toronto, Canada) brightfield illumination microscope (20x/0.8 Plan-Apochromat objective) attached to a Hitachi 3-chip color camera (Hitachi Kokusai Electric Camera, Ltd., Woodbury, NY, USA). All quantification analysis was performed using the HALO® image analysis platform (Indica Labs, 


\section{CHAPTER 5}

Albuquerque, NM, USA). The tumor areas were manually segmented for each stain while excluding histological folding artifacts and non-tumor regions. Using the area quantification module, the CD31 (Figure 5-3b, tumor vascularity) and TUNEL (Figure 5-3e, degree of cell death) positive areas were calculated by separating the stain signal from the background signal. This was accomplished by training the HALO ${ }^{\circledR}$ software on the representative CD31, TUNEL or background intensity levels and automatically generating pseudo colored markups for all slides. In order to quantify the size of each CD31 positive 'object' (defined either as a cluster of endothelial cells or a vessel with the lumen intact, refer to Supplementary figure 5.B), the perimeter of all objects was automatically traced, and a histogram distribution was generated (Figure 5-3c). The mode of the histogram was used to compute the average vessel perimeter. The ratio of the area under the curve to the tumor area was used to compute the vascular density of each tumor in units of number of vessels/ $\mu \mathrm{m}^{2}$.

For each treatment group, the percentage of tumor vascularity, vascular density, average vessel size and tumor cell death were computed at 24 hours post-treatment as shown in Figure 5-3. These histological metrics were normalized by computing the percentage change relative to the untreated control group. The PA imaging biomarkers were correlated against the histological metrics of treatment response in order to assess the relation between the changes in the imaging biomarkers and the biophysical changes that tumors undergo post-treatment. Correlation plots were also generated between relevant histological metrics (vessel perimeter vs. vascular density) and imaging biomarkers ( $\mathrm{HbT}$ vs. $\mathrm{sO}_{2}$ ) for every treatment group. 


\section{CHAPTER 5}

(b)
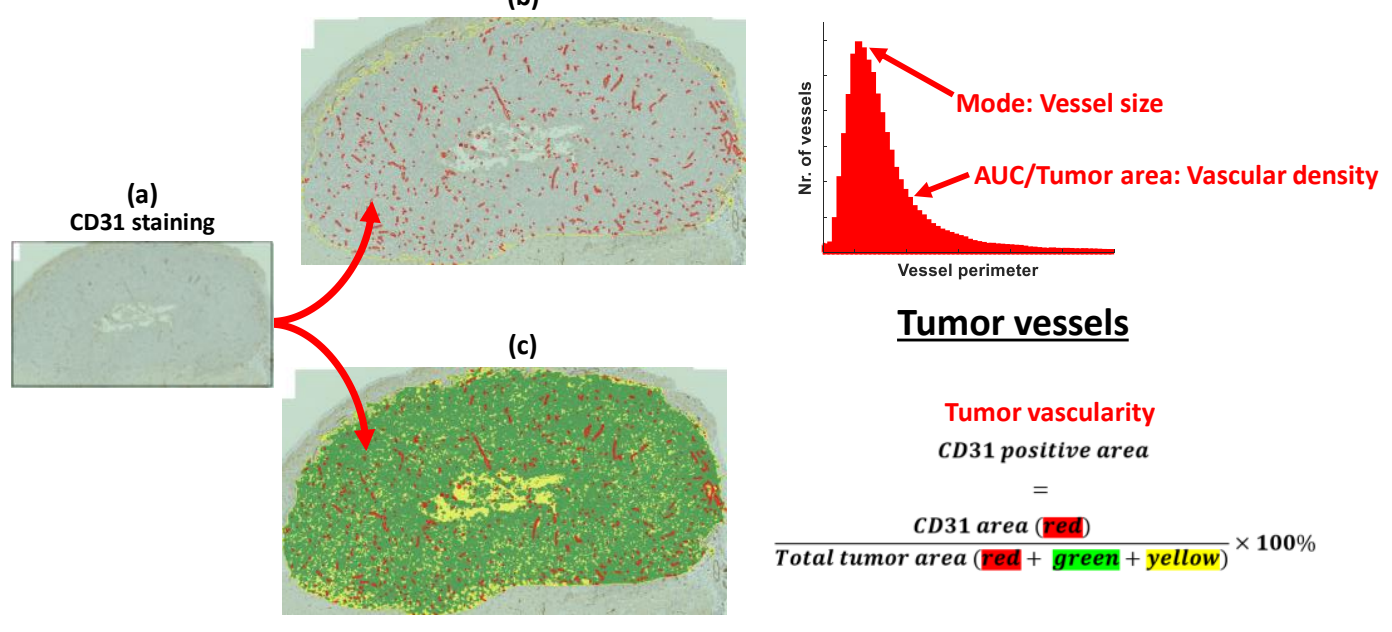

$\underline{\text { Tumor vessels }}$

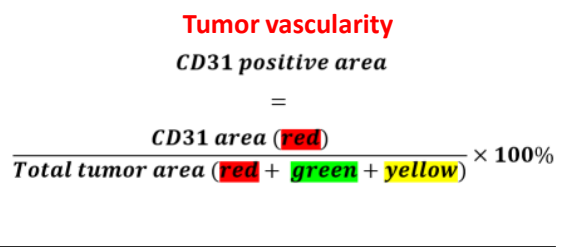

(e)
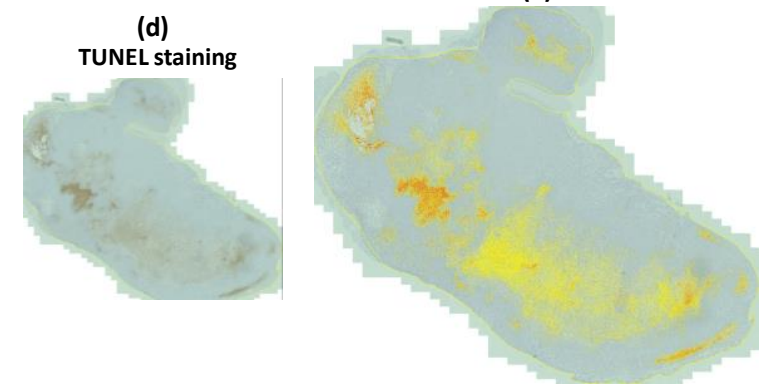

\section{$\underline{\text { Tumor cells }}$}

Degree of cell death

TUNEL positive area

$\frac{\text { TUNEL area }(\text { red }+ \text { yellow })}{\text { Total tumor area }(\text { red }+ \text { yellow }+ \text { grey })} \times 100 \%$

Figure 5-3: Histological metrics of tumor vessels and cells. (a) CD31 staining of tumor blood vessels can be used to estimate both the (b) vascularity of tumors by measuring the CD31 positive area and (c) the vascular density and vessels size by computing the total number and size of each vessel, respectively. The red pseudo color denotes the vessels and green and yellow denote the background and tissue-free areas, respectively. The cell-free areas are assessed by the presence of the hematoxylin nuclear counterstain. (d) TUNEL staining can be quantified by measuring the (e) TUNEL positive area in the tumor in order to estimate the extent of cell death. Red and yellow pseudo colors represent different intensities of TUNEL staining. Abbreviations: CD31 = cluster of differentiation 31; AUC = area under curve; TUNEL = Terminal deoxynucleotidyltransferasemediated-dUNP-biotin nick end labeling.

\subsubsection{Statistical analysis}

A two-way analysis of variance (ANOVA) test was utilized to determine if the changes in $\mathrm{sO}_{2}$, CD31 and TUNEL among treatment groups were statistically significant and whether the MB/NB treatments had an effect on the radiation treatment. A variable with a $p$ value of 0.05 was considered statistically significant and post hoc comparisons using Tukey's least significant differences identified $95 \%$ confidence intervals to determine the pairs which were significantly different [298]. All statistical analysis was performed using Matlab 2018b (The MathWorks, Inc., Natick, MA). 


\section{CHAPTER 5}

\subsection{Results and discussion}

\subsubsection{Quantification of micro/nanobubble treatment response}

Figure 5-4 summarizes the PA-based and histology-based biomarkers post-treatment. The $\mathrm{sO}_{2}$ at 2- and 24-hours post-treatment was compared against the untreated control mice (Figure 5-4a). All changes relative to the control mice are statistically significant at both timepoints $(p<0.05)$. A single fraction of $8 \mathrm{~Gy}$ increases the tumor oxygen saturation by as much as $10 \%$ at 2 hours posttreatment, with a subsequent drop in $\mathrm{sO}_{2}$ at the 24-hour timepoint that is still above pre-treatment levels $(p<0.05)$. The oxygen consumption is driven by the metabolic demands of tumor cells with blood flow regulating the oxygen supply [71]. Radiation-induced vascular changes will affect the tumor $\mathrm{sO}_{2}$ [136] and disrupt endothelial cells [70]. The increase in CD31 staining observed for the 8 Gy group (Figure 5-4b) is consistent with an $\mathrm{sO}_{2}$ increase (Figure 5-4a) and is supported by other studies of tumor neovascularization observed post-radiation treatments [299]-[301]. The acute inflammatory response which is triggered immediately post radiation-induced cellular damage (Figure 5-4c) may increase the blood flow to the tumor, increasing the oxyhemoglobin concentration within the tumor, thereby increasing the $\mathrm{sO}_{2}$.

Czarnota and colleagues have demonstrated that MB+US treatments can be used as radiationenhancing antivascular agents [291]-[293]. Endothelial cells lining the tumor blood vessels undergo apoptosis through the release of ceramide in response to radiation exposure [293]. In addition, the ultrasonic-mediated, inertial cavitation of MBs induces vascular damage to the tumors. These vascular effects can be measured through $\mathrm{PA} \mathrm{sO}_{2}$ estimates as shown in Figure 5-4a and the CD31 staining in Figure 5-4b. The decrease in CD31 staining is most likely related to the reduced vessel integrity, and thus blood flow to the tumor [292], leading to a decrease in the supply of oxygenated blood and thus, oxygenated hemoglobin. As the PA imaging biomarkers are sensitive to the oxygenation of the red blood cells, it can measure this treatment-induced vascular effect.

NBs have shown promise as contrast agents [285]. This study presents the first time nanobubbles are used as vascular disrupting agents. As shown in Figure 5-4a and Figure 5-4b, the NB+US treatments induce a vascular effect that is stronger than the MB+US treatments $(10.3 \%$ in tumor 


\section{CHAPTER 5}

$\mathrm{sO}_{2}$ and $10 \%$ drop in $\mathrm{CD} 31$ compared to $7.7 \%$ and $4 \%$ for $\mathrm{MBs}$, respectively). This suggests that the $\mathrm{NB}+\mathrm{US}$ exposure impacts the tumor vascularity in a manner similar to MBs, possibly inducing damage to blood vessels due to inertial cavitation in the vicinity of endothelial cells.

(a)
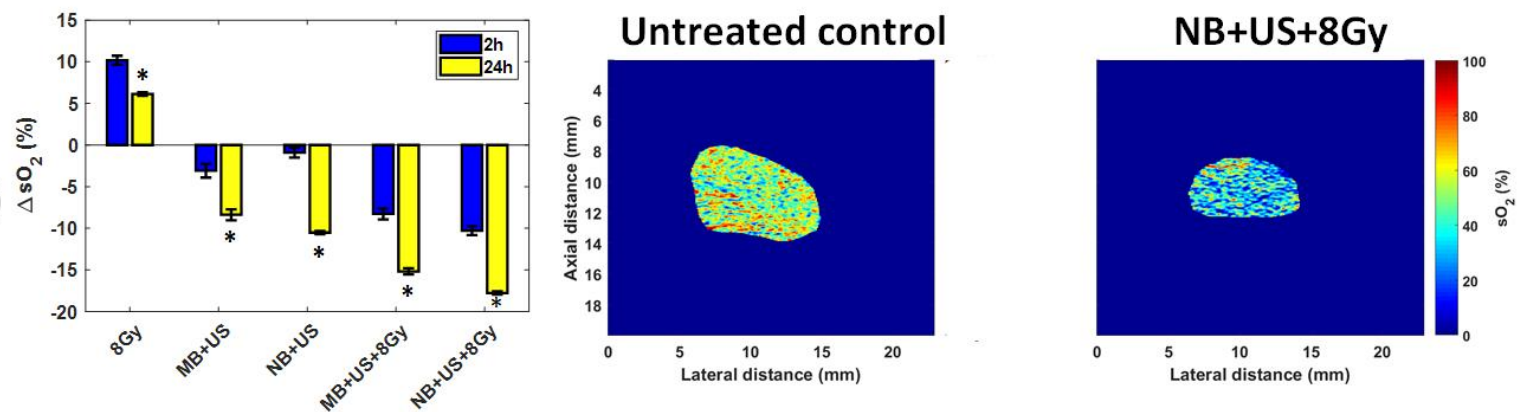

(b)
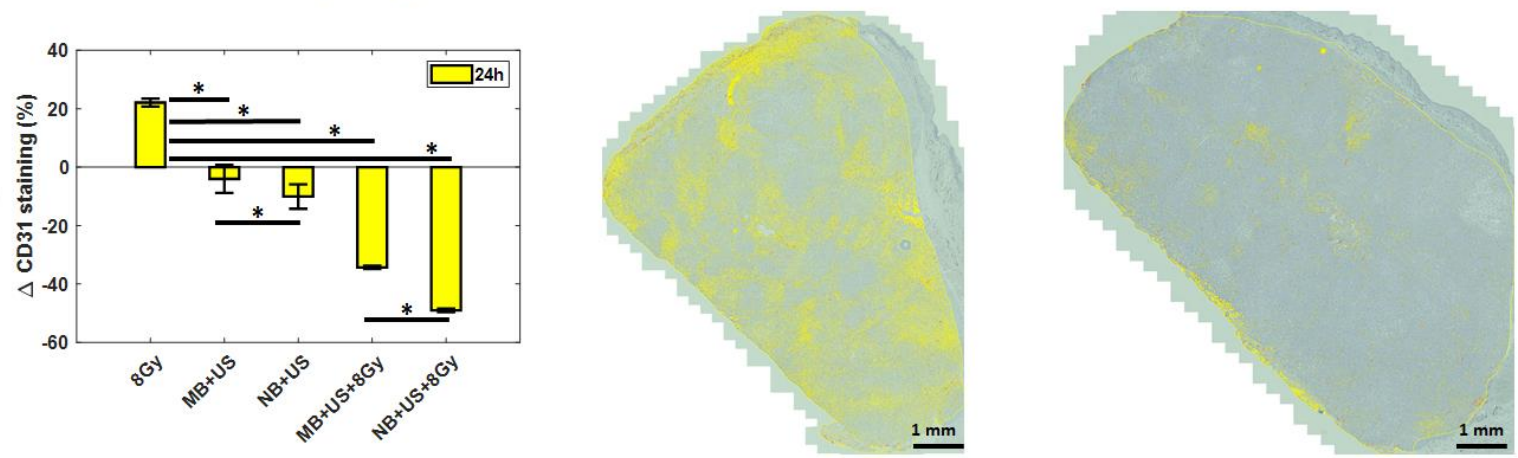

(c)
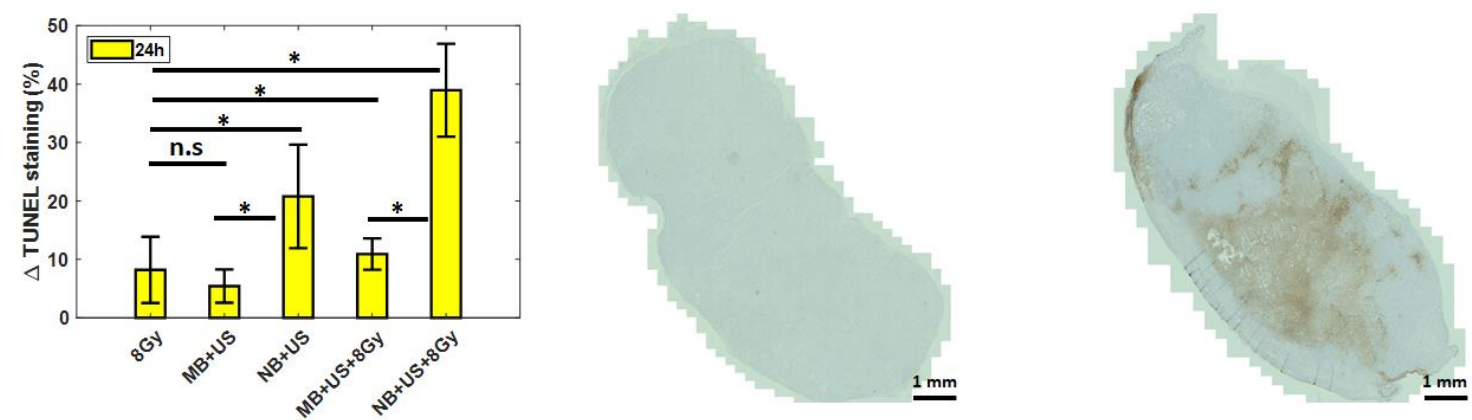

Figure 5-4: PA imaging and histological biomarkers of treatment response. Change in (a) PA estimations in tumor sO2, (b) CD31 positive area and (c) TUNEL positive area relative to the untreated control as a function of treatment and imaging timepoint ( $\mathrm{sO} 2$ only). The error bars denote the standard error of the mean change for all animals belonging in that treatment group. Statistical significance is doted by $*(\mathrm{p}<0.05)$ and n.s. denotes not significant. The sO2 comparisons denote statistical significance between the $2 \mathrm{~h}$ and $24 \mathrm{~h}$ imaging timepoints. For every biomarker, representative sO2 maps, CD31 and TUNEL histological images are shown for the untreated control and the $\mathrm{NB}+\mathrm{US}+8 \mathrm{~Gy}$ treatment. Axial and lateral distances in the $\mathrm{SO} 2$ maps are in relation to the imaging transducer.

The combination of radiation with the MB and NB treatments amplifies the decrease in tumor oxygenation (as assessed by $\mathrm{sO}_{2}$ ) and vascularity. The largest decrease in $\mathrm{sO}_{2}(18 \%)$ was observed 


\section{CHAPTER 5}

for the $\mathrm{NB}+\mathrm{US}+8 \mathrm{~Gy}$ treatment at 24 hours post-treatment (Figure 5-4a) while the tumor vascularity assessed by CD31 staining decreased by nearly $50 \%$ at the same timepoint (Figure $5-4 b)$. The tumor $\mathrm{sO}_{2}$ decreased as early as 2 hours post-exposure for all bubble treatments. The drop in $\mathrm{sO}_{2}$ for the $\mathrm{NB}+\mathrm{US}+8 \mathrm{~Gy}$ treatment was approximately $10 \%$ greater compared to the $\mathrm{NB}+\mathrm{US}$ treatment at both 2- and 24-hours post-treatment. These changes were also mirrored by changes in the CD31 staining (Figure 5-4b), all of which are significantly lower than the single 8 Gy fraction of radiation. These findings support the hypothesis that ultrasonically simulated bubbles impact the vasculature of tumors [291].

Histological analysis of tumors post-treatment offers insights into the mechanism of action for each treatment. Figure 5-4c shows the degree of cell death as a result of radiation, MB and NB treatments. As observed by the representative images of TUNEL staining for untreated control and $\mathrm{NB}+\mathrm{US}+8 \mathrm{~Gy}$ treatments, there is a notable increase in brown staining. Quantification of this apoptotic stain reveals more than 2-fold higher degree of cell death for the NB+US group compared to the MB+US counterpart $(p<0.05)$. This observation suggests that the NBs, for these ultrasound exposure parameters and bubble concentrations, are more effective at damaging the tumor cells than MBs, perhaps due to their ability to extravasate into the tumor interstitium [294]. When combined with radiation, the $\mathrm{NB}+\mathrm{US}+8 \mathrm{~Gy}$ treatment exhibited, on average, $40 \%$ more cell death compared to the untreated control 24 hours post-treatment. Apart from the MB+US treatment, all other treatments had a significantly higher degree of cellular death compared to the single fraction of radiation $(p<0.05)$. The vascular disruption from the cavitation of MBs and NBs leads to a drop in the tumor $\mathrm{sO}_{2}$ as measured by PA imaging. When accompanied by the radiation damage, this leads to an increase in tumor cell death.

\subsubsection{Changes in functional biomarkers during treatment}

The tumor vessel oxygenation has been previously showed as a reliable biomarker of cancer treatment response [133], [136], [150]. In order to further explore the biophysical mechanism of the MB and NB treatments, Figure 5-5 shows a direct comparison between PA biomarkers of tumor oxygenation $\left(\mathrm{sO}_{2}\right.$ and $\left.\mathrm{HbT}\right)$ and histologically measured biomarkers of tumor blood vessels (CD31) and cell death (TUNEL). Simultaneous $\mathrm{sO}_{2}$ and $\mathrm{HbT}$ measurements in the same imaging plane reveal an inverse relationship between the two (Figure 5-5a). As the oxygenation of tumors 


\section{CHAPTER 5}

decreases, there a significant increase in the concentration of total hemoglobin present in the tumor. A nearly $20 \%$ decrease in tumor $\mathrm{sO}_{2}(\mathrm{NB}+\mathrm{US}+8 \mathrm{~Gy})$ is accompanied by nearly $30 \%$ increase in HbT. Total hemoglobin has been shown to correlate with the overall blood volume in biological tissue [146], [223], [279], [302]. In the case of MB- and NB-enhanced radiation treatments, the disruption of the vasculature might lead to the hemorrhaging of red blood cells in the tumor interstitium. The decrease in CD31 staining when the tumor $\mathrm{sO}_{2}$ decreases (Figure 5-5b) and the HbT increases (Figure 5-5c) also supports this hypothesis. These results also suggest that there is a threshold of CD31 staining ( 20\%), beyond which the vascular damage induces an increase in $\mathrm{HbT}$, potentially due to hemorrhaging. This may be probed further through dose-dependent studies in both MB/NB concentration and radiation dose [292].

(b)

(a)

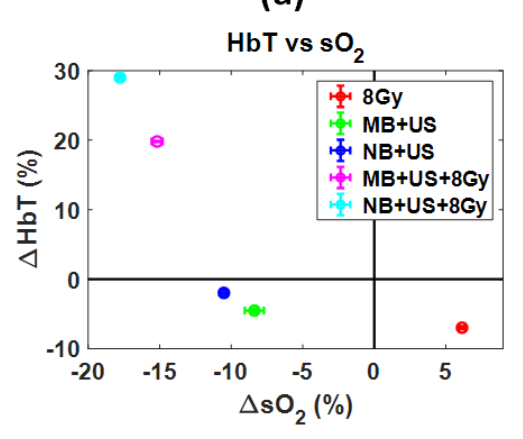

$\mathrm{CD} 31 \mathrm{vs} \mathrm{s}_{2}$

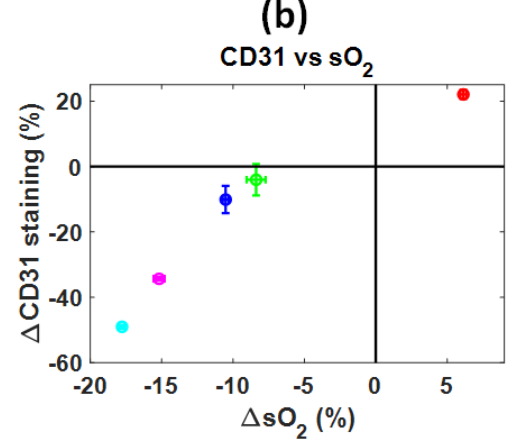

(d)

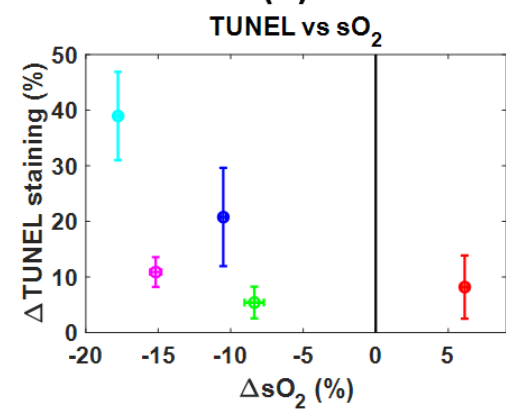

(c)

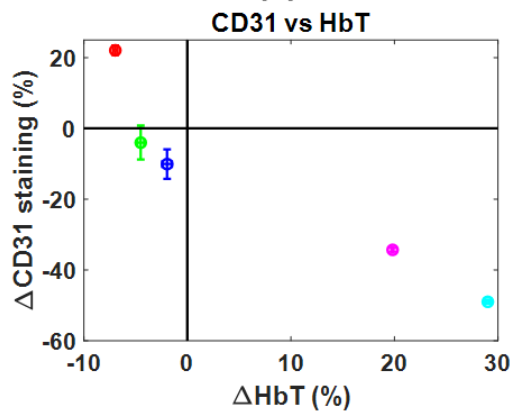

(e)

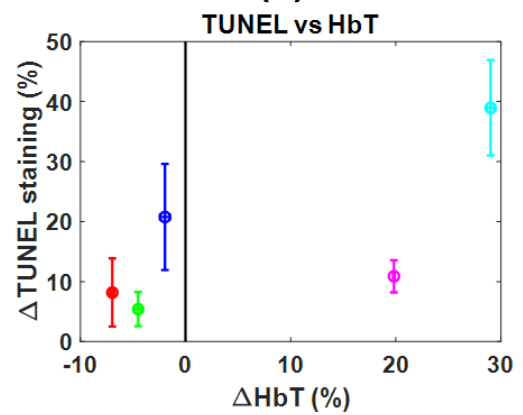

Figure 5-5: Impact of treatments on oxygenation metrics. Correlations between the changes in (a) $\mathrm{HbT}$ and sO2, (b) CD31 and sO2, (c) CD31 and HbT, (d) TUNEL and sO2, and (e) TUNEL and $\mathrm{sO} 2$. Each parameter change is measured at 24 hours post-treatment and is calculated relative to the untreated control, for all treatment groups. Error bars denote the standard error of the mean change for all animals belonging in that treatment group.

We then examined the relationship between tumor cell death (as assessed by TUNEL staining), the tumor $\mathrm{sO}_{2}$ (Figure 5-5d) and HbT (Figure 5-5e). The tumors which exhibited the largest degree of cellular death were also the ones with the largest drop in oxygenation and the largest increase 


\section{CHAPTER 5}

in total HbT. Much like the changes in tumor vascularity (as assessed by CD31 staining), the functional PA-measured biomarkers are correlated with the degree of cell death. NB cavitation can affect the integrity of the endothelial cells, modifying the tumor vascularity. Their extravasation into the tumor interstitium allows them to be in close proximity to the tumor cells. Ultrasonic caviation of gas filled bubbles has been shown to induce cellular death through sonoporation and related mechanisms [295], consistent with the hypothesis that NBs can act as anticancer agents. Figure 5-6 supports these findings by plotting the tumor size after NB-derived treatments. The $\mathrm{NB}+\mathrm{US}+8 \mathrm{~Gy}$ treatment yielded the largest growth delay, observable as early as 12 days posttreatment where a $26 \%$ decrease in tumor volume was recorded. The ultrasound-activated nanobubble treatments and the radiation alone treatment also delay the growth of the tumor in comparison to the untreated control. By day 30 post-treatment, the NB+US+8Gy had suppressed the tumor volume by more than $70 \%$. These observations are consistent with previously published results describing the growth delay effects of US+MB+8Gy treatments [292]. In order to examine the efficacy of NB-mediated radiation therapy in comparison to the MB equivalent treatment, rigorous comparisons must be conducted and are planned for future work. For instance, one must equalize the gas volume encapsulated within MBs and NBs in order to directly compare them [303], [304].

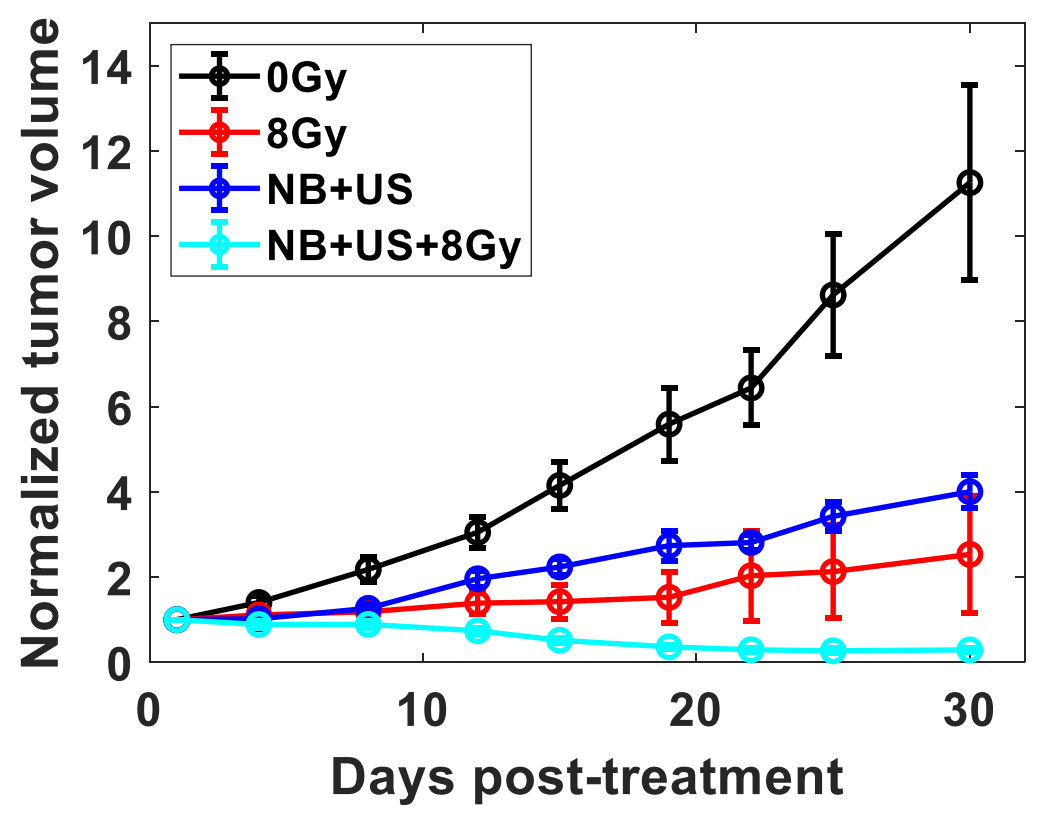

Figure 5-6: Tumor growth delay data for single nanobubble and radiation treatments. The tumor volumes were normalized to the size of the tumor on day 1 post-treatment, for each respective group. The error bars denote the standard error of the mean. 


\section{CHAPTER 5}

\subsubsection{Changes in structural biomarkers during treatments}

Our group has demonstrated that PA imaging biomarkers can be used to study tumor blood vessels [151], [281]. MB and NB treatments appear to impact the structural integrity of tumor vasculature [293]. Figure 5-7 summarizes the histologically measured structural vessel parameters (perimeter and density) as a function of the PA-measured biomarkers ( $\mathrm{sO}_{2}, \mathrm{HbT}$ and $\left.\mathrm{SS}\right)$. The vascular density after the MB and NB treatments decreases, while the vascular density after the 8 Gy radiation dose increases (Figure 5-7a). However, the perimeter of vessels (assessed by CD31 staining, Supplementary figure 5.B) is higher for MB treatments compared to NBs. The addition of radiation decreases vascular density for both the $\mathrm{MB}+\mathrm{US}+8 \mathrm{~Gy}$ and $\mathrm{NB}+\mathrm{US}+8 \mathrm{~Gy}$ treatments when compared to MB+US and NB+US, respectively.

The histologically measured changes in the tumor vascular network can be also probed through PA biomarkers. Specifically, the vascular density is correlated with the tumor $\mathrm{sO}_{2}$ (Figure 5-7b) and $\mathrm{HbT}$ (Figure 5-7c). The nearly $20 \%$ drop in tumor $\mathrm{sO}_{2}$ for the $\mathrm{NB}+\mathrm{XRT}+8 \mathrm{~Gy}$ group is likely due to the destruction of blood vessels. This is supported by an $80 \%$ drop in the vascular density (Figure 5-7b). Moreover, as blood vessels lose their integrity, hemorrhaging of the red blood cells creates interstitial blood pooling [135], [151]. This in turn causes the total hemoglobin in the tumor to increase by nearly $30 \%$ (Figure 5-7c) as blood flowing into the tumor leaks out and accumulates outside of damaged vessels.

Our group and others have demonstrated experimentally and through simulations that the PA spectral slope is sensitive to the size of the source of PA signals [281], [297], [305]. Here we examine how the SS may be used to characterize the changes in vessel size post radiation, MB and NB treatments. Figure 5-7d and Figure 5-7e summarize the changes in SS and vessel perimeter at $750 \mathrm{~nm}$ and $850 \mathrm{~nm}$ illuminations, respectively. Much like the histologic metrics shown in Figure 5-7a, the PA SS can also be used to differentiate between the effects MB and NB-based treatments. Supplementary figure 5.C plots the absolute values of the pre-and post-treatment SS values reflecting the changes observed in Figure 5-7.

The SS decreases at both illumination wavelengths for both the MB+US and NB+US groups. A decrease in the SS has been previously linked to an increase effective absorber size as was shown 


\section{CHAPTER 5}

through simulations and experiments in Chapter 2. In the context of vascular targeted treatments, we have shown that when tumors hemorrhage, the effective absorber size (i.e. pools of red blood cells) increases [151], [306]. The SS and vessel perimeter changed post-treatment further provide evidence that both the MB and NB treatments induce changes in the tumor vasculature. Using CD31 staining post-treatment, one can assess the size of the remaining vessels (i.e. endothelial cells which we hypothesize survived the MB/NB disruption). Figure 5-7d and Figure 5-7e show the changes in SS and vessel perimeter. The perimeter of the remaining vessels after the MB treatments is larger than the perimeter before treatment, whereas perimeter of the remaining vessels after the NB treatments is smaller than before the treatment. For both treatments, the vascular density is significantly reduced. Moreover, the SS drop for the tumors exposed to MB treatments is smaller than for the NB treatments. This SS difference between treatments suggests that the MBs and NBs affect different levels of the tumor vasculature, most likely due to the different size of the MBs $(3 \mu \mathrm{m})$ compared to NBs $(200 \mathrm{~nm})$.

(b)

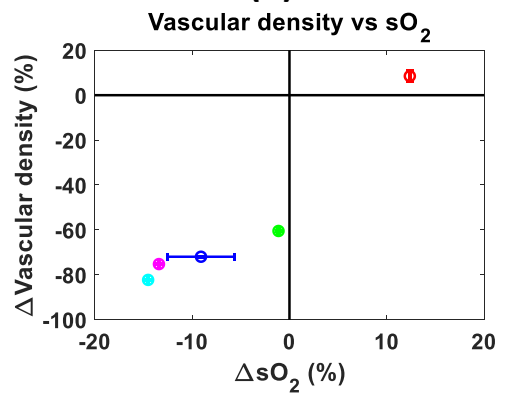

(d)

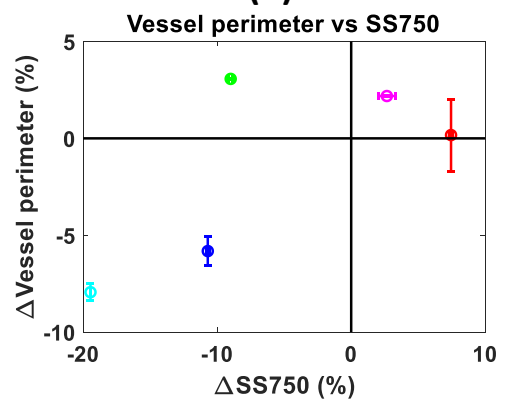

(c)

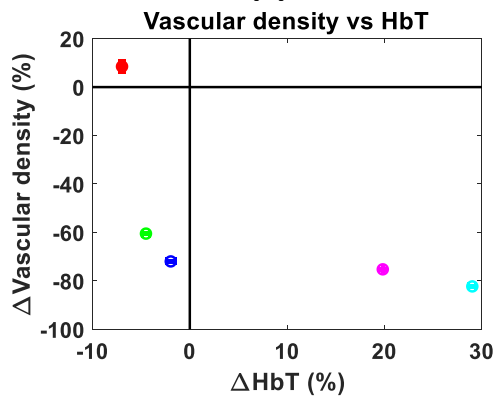

(e)

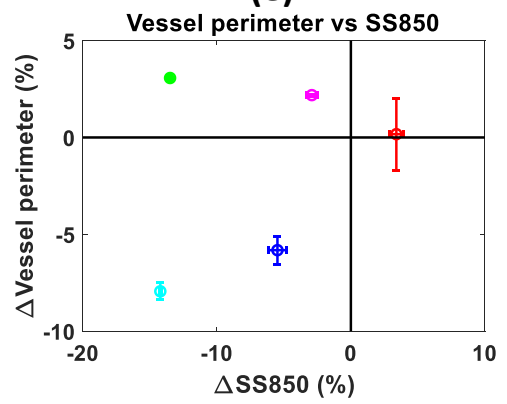

Figure 5-7: Impact of treatments on tumor vascularity. Correlations between the changes in (a) Vessel perimeter and Vascular density, (b) Vascular density and sO2, (c) Vascular density and $\mathrm{HbT}$, (d) Vascular density and SS at $750 \mathrm{~nm}$ and (e) Vascular density and SS at $850 \mathrm{~nm}$. Each parameter change is measured at 24 hours post-treatment and is calculated relative to the untreated control, for all treatment groups. Error bars denote the standard error of the mean change for all animals belonging in that treatment group. 


\section{CHAPTER 5}

Moreover, differences in the changes in the SS arise between the 750 and $850 \mathrm{~nm}$ illuminations for both NB and MB treatments. At $750 \mathrm{~nm}$, the decrease in spectral slope for the NB treatments is greater than at $850 \mathrm{~nm}$. For $750 \mathrm{~nm}$, the wavelength for which deoxygenated hemoglobin has a higher molar extinction coefficient [147], the decrease in spectral slope is greater. This might be an indication of larger pools of deoxygenated blood due to hemorrhaging. The decrease in both vessel perimeter and CD31 staining for NB treatments would support the hemorrhaging hypothesis, especially when considering the increase in HbT with the treatments with radiation.

Conversely, for the MB treatments, at $750 \mathrm{~nm}$ the decrease in spectral slope is less than at $850 \mathrm{~nm}$. At $850 \mathrm{~nm}$, oxygenated hemoglobin has a higher molar extinction coefficient and contributes more to the PA signal, and therefore red blood cells outside the hemorrhagic pool would contribute more to the signal. The increase in the vessel perimeter and decrease in CD31 staining for MB treatments would indicate for the MB treatments there is less hemorrhage compared to the NB treatments. A wavelength dependence in the SS has been observed when monitoring PA sources of different absorption properties such as fat deposits in the liver [297] or benign tumors in ovarian tissues [307]. In this work, the difference between the SS at both wavelengths can be attributed to the oxygen dependence of hemoglobin, the most abundant absorber inside red blood cells [147].

\subsection{Conclusions}

The goal of this work is to use histological metrics of cancer treatment response to understand biomarkers in acoustic resolution photoacoustic imaging and use these metrics to compare the cancer treatment responses to microbubble and nanobubble radiosensitization of the vasculature. This study demonstrates that PA biomarkers can be used to differentiate tumor treatment response to microbubble and nanobubble enhancements of radiation treatments. Biomarkers of tumor oxygenation and vascular structure are directly related to the changes that the blood vessels undergo during radiation-enhancements through bubble stimulation. The nanobubble-radiation combined treatments result in twice as much cellular death as nanobubbles alone and show an enhancement compared to the microbubble counterparts for the exposure parameters and bubble concentrations used in these experiments. These experiments offer a new insight into the use of functional and structural biomarkers of photoacoustic imaging for early cancer treatment monitoring. 


\section{CHAPTER 5}

\subsection{Acknowledgments}

This research was undertaken, in part, through grants provided by the Terry Fox Foundation through a Program Project Grant funded through the Hecht Foundation (GJC and MCK), National Institutes of Health (AAE and MCK) and the Natural Sciences and Engineering Research Council of Canada (MCK). EH held a Vanier Canada Graduate Scholarship during the conduct of this research. MF was supported through an NSERC Alexander Graham Bell Graduate Scholarship. The authors would like to acknowledge the assistance of Dr. Azza Al-Mahrouki, Dr. Lauren Wirtzfeld and Nikki Law. The Keenan Research Centre Core Facilities at St. Michael's Hospital and Dr. Caterina Di Ciano-Oliveira are acknowledged for assistance with histological analysis. The technical support provided by the Toronto office of Fujifilm-VisualSonics is also gratefully recognized. 


\subsection{Supplementary information}

\subsubsection{Supplementary figure 5.A}

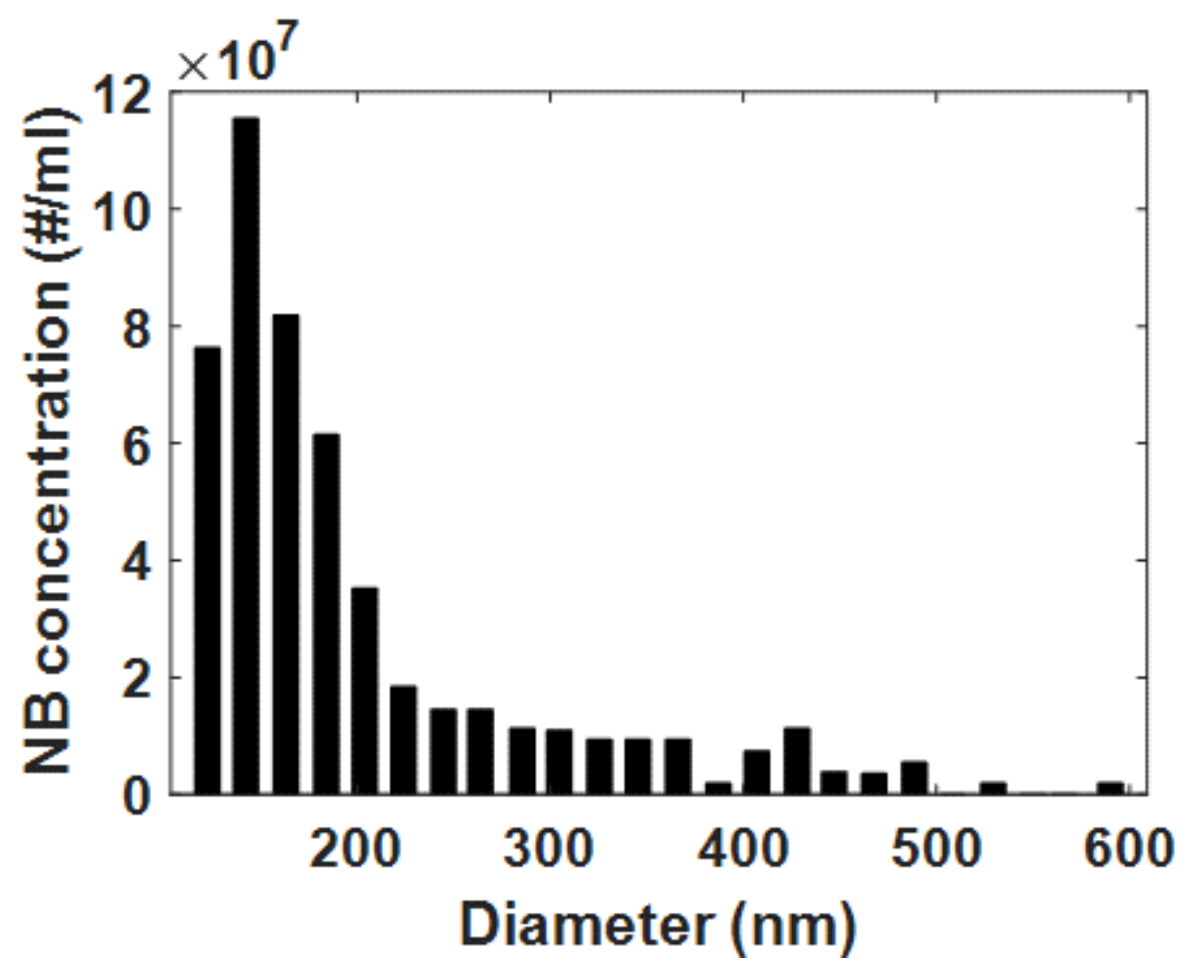

Size distribution of NBs as measured with the Archimedes ${ }^{\circledR}$ device (Malvern Panalytical, Westborough, MA, USA). 


\section{CHAPTER 5}

\subsubsection{Supplementary figure 5.B}

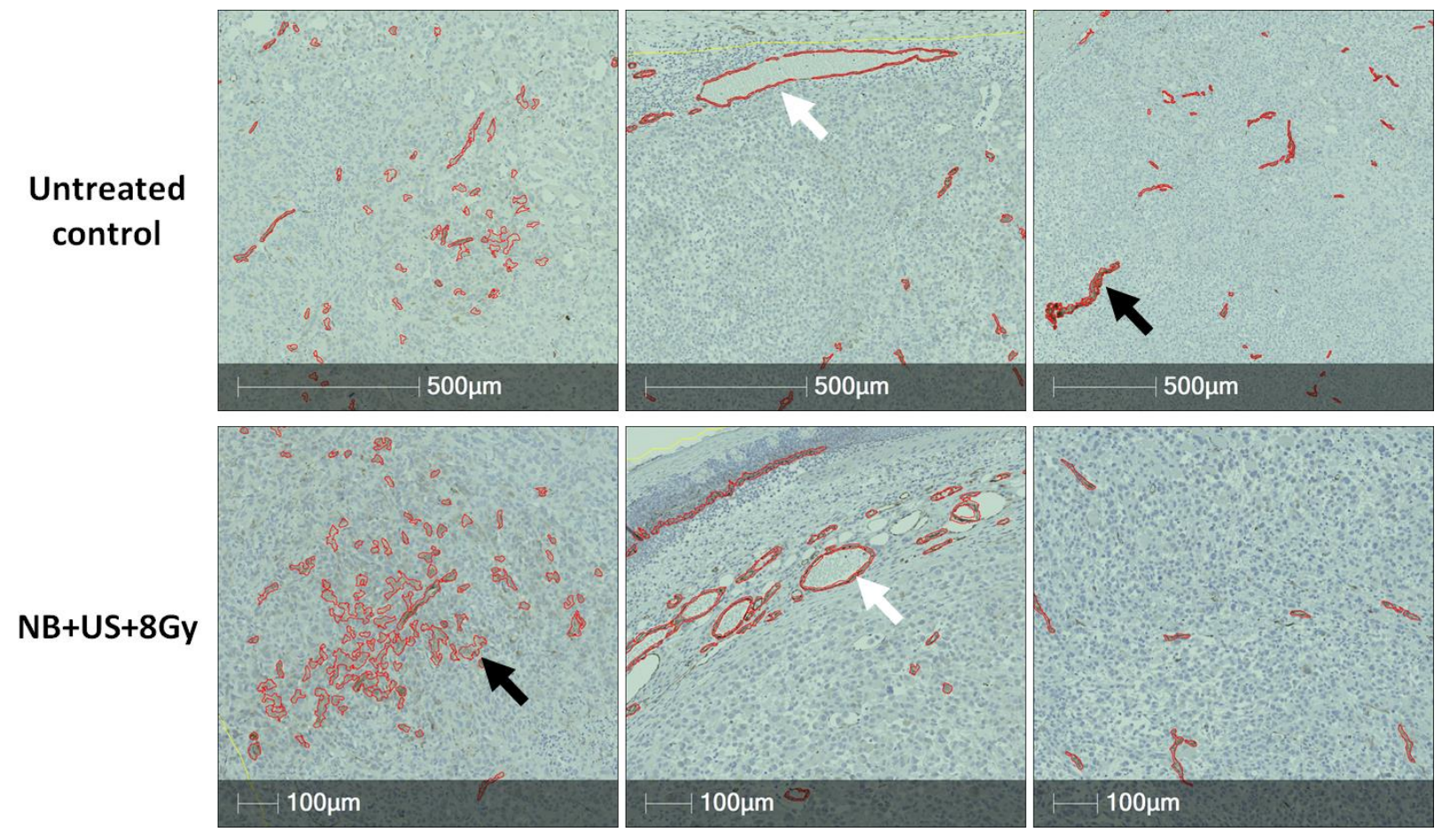

Representative CD31 images for three untreated control and three NB+US+8Gy mouse tumors. The red markups are generated by the HALO® image analysis platform and denote vascular 'objects' which consist of either endothelial cell clusters (black arrows) or vessels with intact lumens (white arrows). 


\subsubsection{Supplementary figure 5.C}

(a)
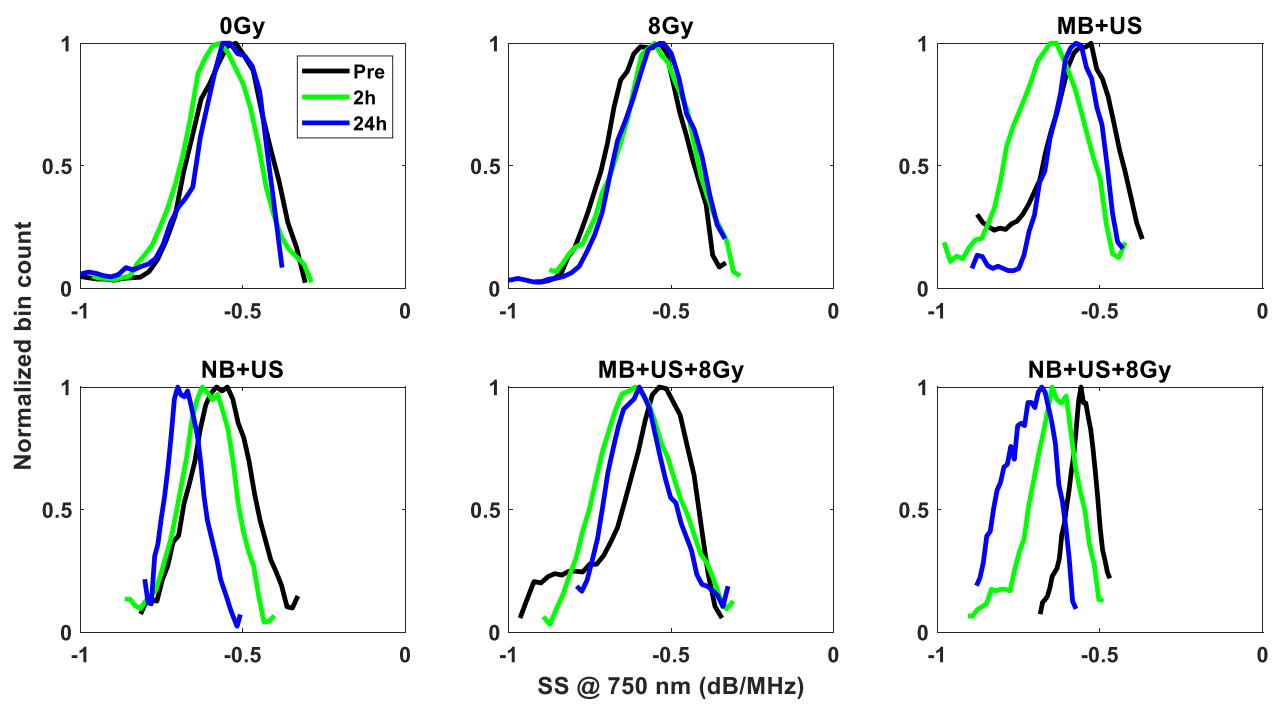

(b)
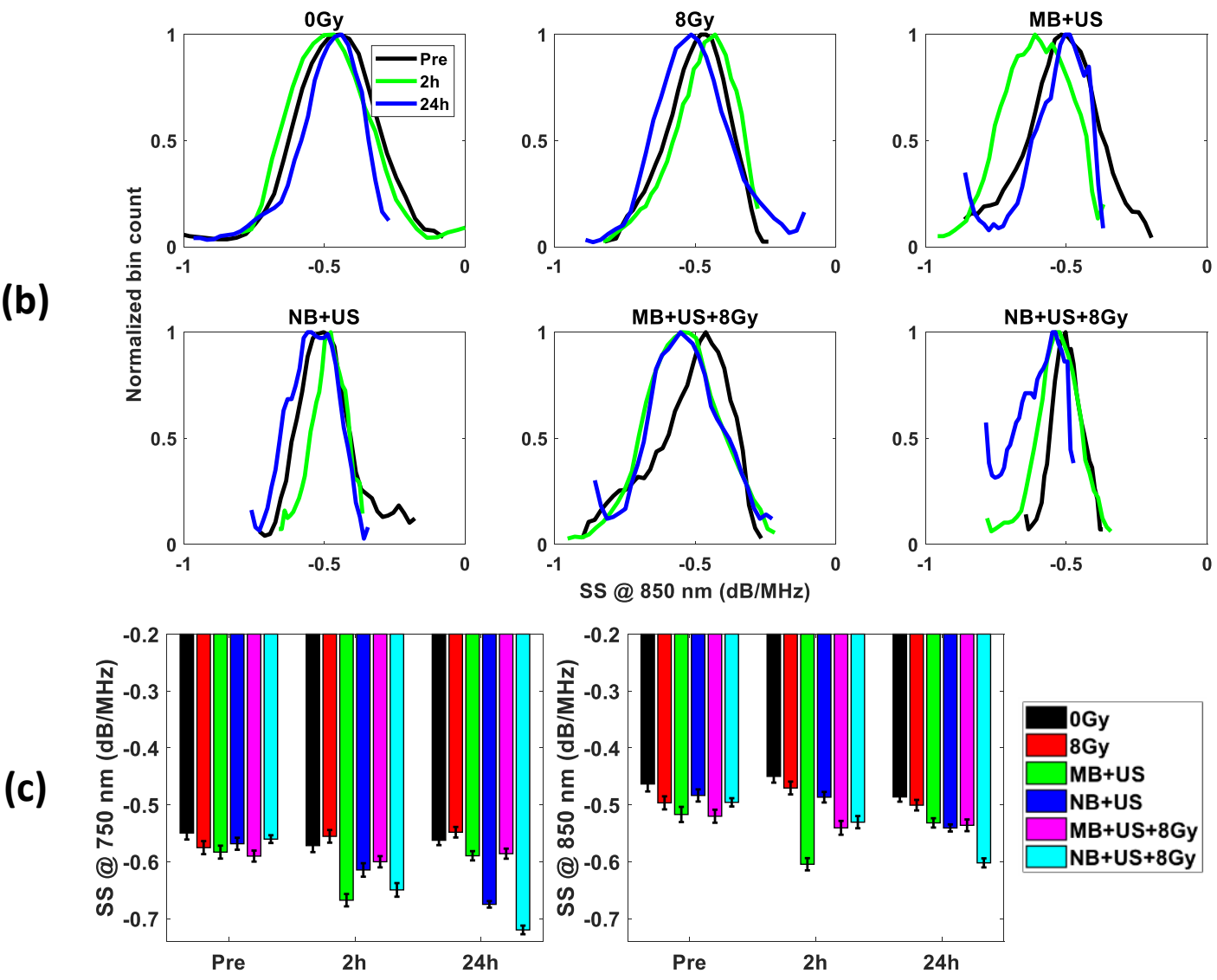

Histogram distributions of the PA SS across the entire tumor volume at (a) $750 \mathrm{~nm}$ and (b) 850 $\mathrm{nm}$ for the pre-treatment, $2 \mathrm{~h}$ and $24 \mathrm{~h}$ post-treatment imaging timepoints for all the mice belonging in that treatment group. The 0Gy represents the untreated control against which all the imaging parameters were normalized against. (c) Average PA SS values for all treatments. The error bars represent the standard error of the mean. 


\section{CHAPTER 6}

\section{Conclusions and future directions}

\subsection{Thesis summary and conclusions}

The work described in this dissertation demonstrates that PA imaging can be used to measure the treatment-induced, structural and functional changes in tumor vasculature. I present a mathematical formulation for the formation of speckle in acoustic resolution PA imaging, that form the foundation for the development of PA biomarkers of treatment response. These structural and functional biomarkers were tested through numerical simulations and in vitro experiments in tissue mimicking phantoms. Subsequently, they were extended to in vivo imaging of pre-clinical mouse breast and prostate tumors. It was shown that PA imaging can be used to monitor the progression of two vascular-targeting treatments through multiparametric quantification of vascular changes. The early (hours post-treatment administration) changes in tumor vessel oxygenation and vessel size can be used to monitor treatment response. This work offers insights towards the clinical translatability of PA imaging, particularly towards the individualized monitoring of vascular-targeted cancer therapies. Below I summarize the main findings and conclusions of each chapter comprising this thesis.

\subsubsection{Formation of PA speckle and the derivations of biomarkers}

1) The laser illumination and ultrasonic detection in acoustic resolution PA imaging permits conditions that allow for the formation of speckle. Speckle arises from the spatiotemporal superposition of the pressure waves from non-resolvable absorbers.

2) PA speckle was previously considered to be interfering noise that degrades image quality. For the first time, I demonstrate theoretically and experimentally that speckle encodes information related to non-resolvable absorbers.

3) Time and frequency domain analysis of RF signals from acoustic resolution PA imaging can be used to investigate changes in the size, shape, number density and spacing between absorbers. In numerical and physical phantoms, the envelope statistics, radiofrequency spectroscopy and cepstral analysis techniques can probe PA absorber concentrations, sizes and periodicities, respectively.

4) These analysis methods form the basis for the development of biomarkers of acoustic resolution PA imaging, to be later used for cancer treatment monitoring. 


\section{CHAPTER 6}

5) Fully developed speckle also arises from PA imaging of the tumor vasculature. This was shown through in silico, fractal-based models of tumor blood vessels and in vivo imaging of mouse breast cancer tumors. The frequency content of the PA signals revealed that a decrease in the spectral slope can quantify the increase in average tumor vessel size, linked to tumor growth. Moreover, the cepstral peaks reveal that the average spacing between cylindrical vessels decreases as tumors grow. This is consistent with the morphological changes tumor blood vessels undergo as a result of angiogenic processes.

\subsubsection{Tumor $\mathrm{SO}_{2}$ changes early after HaT-DOX cancer treatment}

1) The burst-release of doxorubicin encapsulated within HaT-DOX is an effective cancer targeting strategy. It caused a significant hemorrhaging, blood coagulation and vascular shut down of the tumor vasculature. This was due to increased tumoral drug uptake compared to tumors treated with DOX alone or comparable lyso-lipid temperature sensitive liposomes.

2) The acoustic resolution PA images of breast cancer tumors contain speckle, which in turn was observed at multiple optical wavelengths. As such, it encoded functional information about the oxygenation of the tumors. The developed, histogram-based, tumor vessel oxygenation metrics could capture the distributions of $\mathrm{PA} \mathrm{sO} 2$ estimations across the entire tumor volume. This could be used to track the longitudinal oxygenation changes.

3) A drop in the $\mathrm{PA} \mathrm{sO}_{2}$ biomarker is thought to be related to the hemorrhaging of red blood cells in the tumor interstitium. Moreover, the degree of response based on the 2 hours drop in tumor $\mathrm{sO}_{2}$ could also be quantified, with HaT-DOX showing the largest decrease.

4) FITC-lection perfusion levels in the tumor interstitum and the window chamber setup reveal significant bleeding within treated tumors. The vascular damage of HaT-DOX treated tumors at 2 hours post-treatment is significantly higher than the saline-treated control. The vascular damage correlates well with the early drop in $\mathrm{sO}_{2}$, which in turn correlates with the long-term regression of tumors. This demonstrates the usefulness of the $\mathrm{sO}_{2}$ biomarker.

5) A threshold in the tumor $\mathrm{sO}_{2}$ change post-treatment can offer a reliable means of predicting whether a tumor responds to treatment. 


\section{CHAPTER 6}

\subsubsection{Oxygenation and frequency biomarkers can differentiate treatment response}

1) Frequency-domain analysis of PA RF signals is sensitive to sub-resolution changes in tumor vasculature. This enhances the capabilities of PA imaging beyond the resolution limit dictated by the transducer bandwidth.

2) In treatments that disrupt the tumor vasculature such as HaT-DOX, the SS biomarker is the most robust of the frequency analysis variables for monitoring the progression of treatment. It can be used to detect treatment-induced hemorrhaging, which in turn increases the overall effective PA absorber size.

3) The functional $\left(\mathrm{sO}_{2}\right)$ and structural (SS) biomarkers are correlated to one another through the first 5 hours post-treatment. This provided further evidence for the loss of oxygenation due to HaT-DOX-induced vascular destruction.

4) The frequency content of the PA data can be related to the morphological changes that tumor blood vessels undergo during treatment.

5) The combination of $\mathrm{sO}_{2}$ and $\mathrm{SS}$ can identify treatment responders in as early as a few hours post-treatment.

\subsubsection{The biophysical interpretation of PA biomarkers}

1) PA biomarkers of treatment response can be used to elucidate the treatment-induced biophysical tissue changes. They can also be correlated to the gold standard histology stains.

2) Nanobubble-mediated radiation therapy appears to impact the vasculature of tumors and induce cellular death. These changes are observed in PA imaging biomarkers and quantified through gold standard histological examinations.

3) The tumor $\mathrm{sO}_{2}$ biomarker can be used to monitor:

a. The acute inflammatory response triggered from the radiation-induced cellular damage. This is assessed by measuring oxyhemoglobin concentration within the tumor.

b. The vascular damage most likely resulting from the ultrasonic simulations of microbubbles and nanobubbles inside the tumor blood vessels.

c. The enhancement of the vascular damage when radiation is combined with bubble treatments. 


\section{CHAPTER 6}

4) The SS biomarker can be used to monitor:

a. The changes in aggregate vessel size post radiation and bubble treatments.

b. Hemorrhaging as a result of vascular disruption.

c. The oxygenation level of hemoglobin following extravasation of red blood cells into the tumor interstitum post hemorrhaging.

5) There is evidence that the microbubble and nanobubble treatments affect different levels of the tumor vasculature as a result of the order of magnitude differences in their sizes.

\subsection{Significance and impact}

I demonstrated in this dissertation that PA imaging biomarkers derived from the time and frequency domain analysis of RF signals can quantify structural and functional vascular changes during cancer treatments (Figure 6-1). Most importantly, these changes can be used to monitor cancer therapies and potentially predict outcome by relying on early measurements post-treatment. Outside of this work, the potential of PA for cancer treatment monitoring remains largely unexplored. Moreover, I have contributed towards the development of a series of PA biomarkers that enhance the capabilities of PA imaging beyond the resolution limit of the imaging system. In acoustic resolution PA imaging, it is not always possible to visualize structures within the illumination field of view. This creates speckle patterns which are generally thought to be detrimental to the imaging resolution.

In this thesis, I have developed PA imaging analysis methods that extend beyond the conventional approach for dealing with speckle, which is typically its suppression through imaging filters. The theoretical and experimental work discussed in this thesis establish that PA speckle encodes information about the underlying tissue microstructure. This information can be extracted by relying on RF signal analysis in the time and frequency domains. The imaging biomarkers derived from such techniques were used to probe the mechanisms of two separate vascular targeted treatments, namely thermosensitive liposomes and nanobubble-mediated radiation therapy. I showed how treatment induced biophysical changes to the vasculature are linked to the PA imaging biomarkers for both treatments. Even though the action of each treatment is fundamentally different (heat-activation vs. ultrasound excitation), the PA biomarkers derived are markers of vascular damage and could also be used to predict the outcome of each treatment. This is a new 


\section{CHAPTER 6}

contribution to the body of literature and most importantly, it has implications in the management of cancer therapies. Being able to provide oncologists with quantifiable metrics which have a meaningful biophysical interpretation can offer valuable insights into identifying the most efficacious treatments. As standard treatment monitoring relies on anatomical assessments which could take months to manifest, the identification of responders early could improve the overall survival. Additionally, this strategy can also spare patients unnecessary side effects of ineffective treatments.

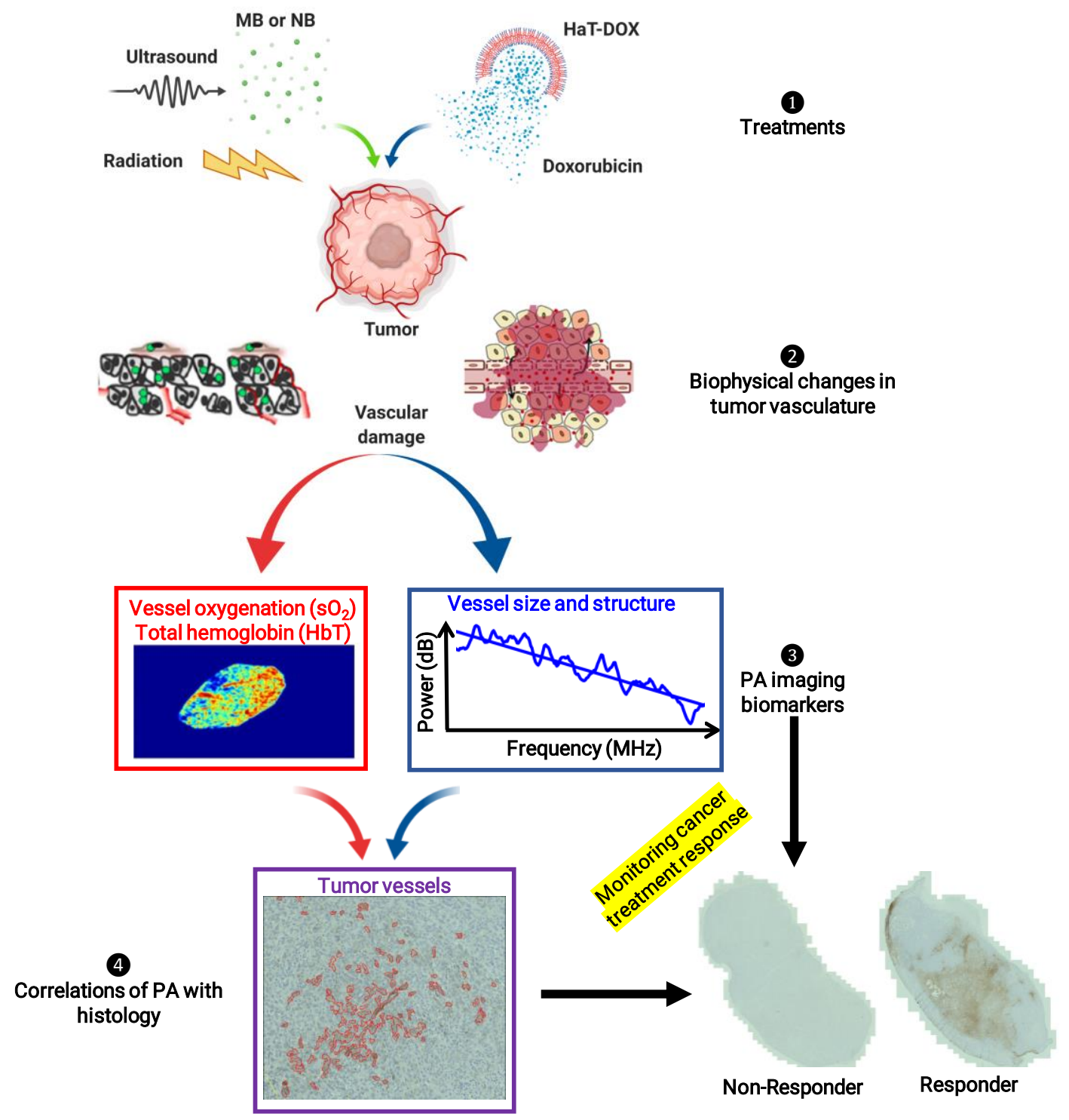

Figure 6-1: Schematic highlighting the work in this thesis. The development of PA imaging treatment biomarkers that induce vascular damages enable monitoring of cancer treatment response. Histological assessments of vasculature and tumor cell death enable the identification of treatment responders. Images were adapted from references [150], [151] and [293]. Illustrations were created with Biorender.com. 


\section{CHAPTER 6}

\subsection{Limitations}

The studies presented in this dissertation improve our understanding of how PA imaging can be used for non-invasive cancer treatment monitoring by quantifying the early changes in the tumor vasculature. As this is one of the first of its kind studies, it has some limitations, which form the basis of potential future extensions of this work. PA imaging depends on the tissue optical properties which impact the propagation of photons [308]. The PA image amplitude depends on the absorption coefficient spectrum and the local light fluence spectrum. The accuracy of the quantification of endogenous chromophores or tagged molecular markers is subject to debate [309]. One must correct for the effect of the tissue optical properties and model the optical scattering and absorption of tissue.

The accuracy of $\mathrm{sO}_{2}$ quantification depends on the choice of illumination wavelength required to probe the oxygen-dependence of the $\mathrm{HbO}$ and $\mathrm{Hb}$ absorption spectra. Regardless of the wavelength selection, endogenous absorbers and scatterers in the surrounding illuminated region (ex. water, lipids or $\mathrm{HbO} / \mathrm{Hb}$ in nearby microvasculature) will change the optical fluence. This effect is known as spectral coloring and is well documented in PA imaging [190]. Challenges remain with performing fluence correction in vivo because a priori information on the spatial distribution of the tissue optical properties are rarely available. A few approaches (including recent attempts by our group) have been proposed to compensate for fluence effects in vivo in order to obtain accurate $\mathrm{sO}_{2}$ estimations [310]-[312]. However, the practical implementation of these approaches remains complex because of the need for estimating the non-uniform light distributions within the tissue, often requiring several approximations [308]. For these reasons, fluence corrections were not applied for the estimations of the $\mathrm{sO}_{2}$ in this work. As such, the computed values of the $\mathrm{sO}_{2}$ used throughout this thesis are not meant to be considered as absolute values and only the relative changes to pre-treatment values are utilized.

As this technology translates towards larger animal models (ex. rabbits) and mainstream radiology, fluence compensation approaches become more important. Specifically, when imaging larger depths of field, illumination schemes involving ballistic photons are utilized as shown in Figure 1-4c). As such, fluence compensation is warranted due to the strong dependence of the light distribution patterns on different optical wavelengths for ballistic photons [190]. Another 


\section{CHAPTER 6}

improvement on the accuracy of the treatment response quantification is the correction in the spectral slope estimations for acoustic attenuation. Due to the one-way travel of acoustic waves in PA imaging, the impact of ultrasonic attenuation is smaller than that in conventional ultrasound imaging [142]. The attenuation compensation could also improve the signal to noise ratio of PA images, thus enabling the development of efficient image reconstruction approaches [313].

Both treatment types utilized in this thesis (HaT-DOX and MB/NB) are non-conventional cancer treatments tested in murine preclinical models of cancer. A commercially available variant of HaTDOX, ThermoDox ${ }^{\circledR}$ (Celsion Corporation, New Jersey, USA) is currently on Phase III clinical trials for hepatocellular carcinoma [314]. The combination of ultrasonically-simulated MB and radiation currently remains in the preclinical stage [315], with plans to transition the treatment in trials of locally advanced breast cancer. The significant vascular changes that accompany these treatments permit the use of PA imaging since the modality is sensitive to the hemoglobin present in red blood cells. These treatments are currently not used in routine clinical practice but are specifically designed to impact the tumor vasculature.

One must test whether PA can detect vascular changes from clinical treatments that are designed to target cancer cells or other components (ex. chemotherapy, radiation therapy, immunotherapy, hormone therapy, bone marrow transplants, targeted drug therapy). PA imaging feasibility must be established in monitoring conventional treatments or combinations with experimental therapies. This is especially important in larger animal models of cancer such as rabbits or pigs where the presence of an immune system will have an impact on outcome. Moreover, although the sex of the animals was matched to the cancer cell line (PC3 prostate cancer cell line in male mice and EMT6 breast cancer cell line in female mice), the age of the mice at the time of tumor inoculation could be better chosen. The mice were inoculated at a 5-6 weeks old which corresponds to the puberty phase of the animal's development [316]. To increase the clinical predictability of such mouse models on human diseases, tumors that grow in adult mice might more accurately model the tumor microenvironment of cancer patients [317], [318].

In this thesis, acoustic resolution imaging was performed at two frequencies $21 \mathrm{MHz}$ and $40 \mathrm{MHz}$. This high frequency imaging permits the interrogation of the tumor microvasculature on the order 


\section{CHAPTER 6}

of the transducer spatial resolution $(45-130 \mu \mathrm{m})$. However, the frequency also limits the field of view which is $23 \mathrm{~mm}$ wide $\times 30 \mathrm{~mm}$ depth at $21 \mathrm{MHz}$ and $14 \mathrm{~mm}$ wide $\times 15 \mathrm{~mm}$ depth at $40 \mathrm{MHz}$. This restricts the ability of the technique to image non-superficial tumors such as the subcutaneous tumors used in this thesis. The use of lower acoustic frequencies will permit imaging of tumors in a clinical setting as has been recently shown for breast cancers [106], [108]. In addition, improvements of the penetration depth is also dependent on the optical illumination schemes [93]. The impact of other types of illumination configurations on the quantification of vascular changes in tumors remains to be investigated. This is particularly important for those geometries where PA speckle is suppressed as is the case in tomographic or optical resolution PA systems [245].

Lastly, a limitation of this dissertation lies in the histological analysis performed. Only representative slices were retrieved from the tumor volume, staining for several different types of histological markers. The slices were taken from the largest cross section of the tumor, in the same direction as the axial imaging plane. This potentially biases the histological analysis as it does not account for the intra-tumoral variations in treatment response. As PA imaging was performed in 3D, it might be possible to carry out one-to-one spatial correlations with the stains if whole-mount histopathology is acquired. This would strengthen PA imaging's role as a modality that can be used to study the treatment-induced biophysical changes inside tumors.

\subsection{Future directions}

The directions presented here offer further extensions of this work with the goal of advancing the clinical translation of PA imaging by developing robust biomarkers of treatment response.

\subsubsection{Blood flow and metabolic oxygen consumption measurements}

The microcirculation plays a crucial role in supplying nutrients and removing waste products during tumor growth through angiogenesis. It also provides a pathway for cancer cells to metastasize to distant organs or tissues [319]. Moreover, the efficacy of radiotherapy treatments depends on the local oxygen concentration which is ultimately governed by the local blood flow. In chemotherapy or other types of systemic therapies, the flow of blood is an important determinant for the delivery of the therapeutic payload. In hyperthermia treatments, the blood perfusion rates impact the temperature distribution inside tumors. The rapid proliferation of tumor cells prevents 


\section{CHAPTER 6}

the modulation of blood flow, changing the oxygen consumption. As a result, hypoxia becomes a common feature of tumors, correlating with poor prognosis [47], [61]. For these reasons, blood flow and by extension, the metabolic rate of oxygen consumption are important indicators of tumor viability and its response to various therapies.

Blood flow is conventionally assessed through Doppler ultrasound with applications in the diagnosis of vascular diseases and tumors [320]. Based on the Doppler effect, the flow of red blood cells can be characterized by measuring the frequency change that is produced when the scatterer moves through the ultrasonic beam. With multiple variants, namely continuous wave, pulsed wave, color and power Doppler, the approach is routinely used clinically to diagnose multiple cardiovascular flow conditions such as deep vein thrombosis or carotid artery stenosis [320]. However, Doppler ultrasound remains primarily limited to measurements of blood flow in large arteries and veins since the backscatter from microvessels is generally weaker. Additionally, microvessels exhibit slower flow speeds, making it difficult to distinguish their movement from that of surrounding tissue [321].

Photoacoustic flowmetry and velocimetry techniques have the potential to overcome the limitations of conventional Doppler by relying on the strong optical absorption of red blood cells compared to the neighboring tissues [322]. PA measurements are made in a similar manner to conventional pulse-echo Doppler ultrasound, namely by recovering the Doppler frequency, phase, or time shift which is encoded in the PA waves emitted by flowing red blood cells [97], [323]. The biggest advantage of PA Doppler is the fact that unlike Doppler ultrasound, the acoustic signal recorded is directly emitted by red blood cells rather than being weakly scattered by them [324]. PA Doppler can therefore be used in its own right to study flow in tumor blood vessels. As the tortuous nature of tumor blood microvasculature leads to highly variable and chaotic blood flow patterns [59], [76], [135], [319], it inhibits therapeutic response. Moreover, as PA imaging is sensitive to the oxygen-dependence of the hemoglobin inside red blood cells, it is possible to estimate the metabolic rate of oxygen consumption inside tumors. The latter is defined as the total amount of oxygen delivered to tissue per unit time minus the amount of oxygen leaving the same region of interest per unit time [325]. This estimation depends on the $\mathrm{sO}_{2}$, mean flow speed and the cross-sectional area of blood vessels. All of these parameters that can be assessed by PA 


\section{CHAPTER 6}

imaging as shown in Figure 6-3 for photoacoustic microscopy applications. It would be advantageous to longitudinally map tumoral flow patterns along with the oxygen consumption. The development of these biomarkers for acoustic resolution PA imaging is still in its infancy [321] and tumor applications of blood flow remain unexplored. In conjunction with the structural and functional biomarkers of tumor vasculature developed in this thesis, one would be able to obtain a full snapshot of the tumor microenvironment, thus providing a multifaceted view of the progression of cancer treatments.

(a)

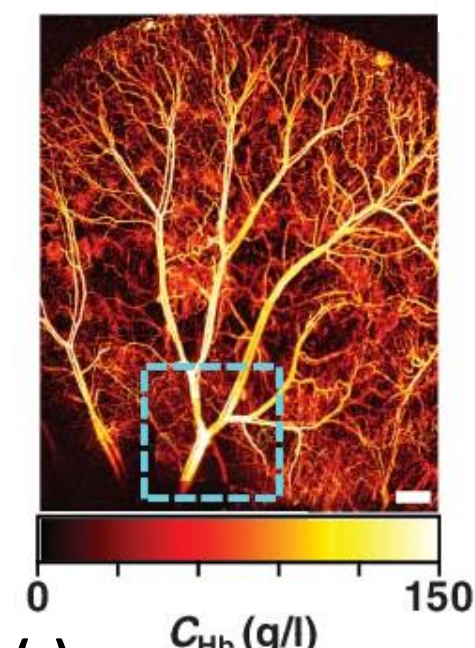

(c)

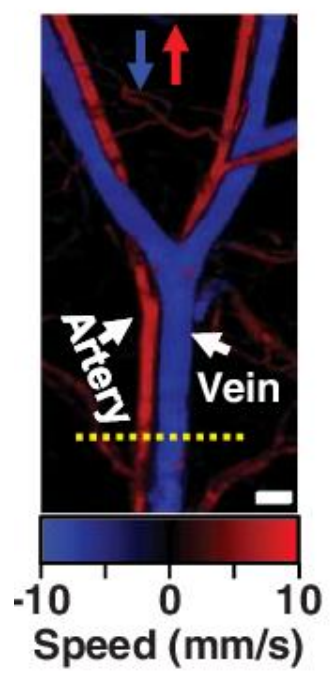

(b)

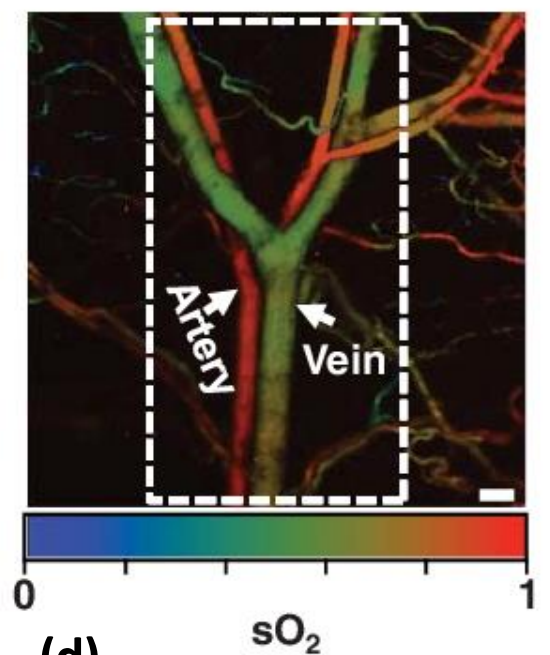

(d)

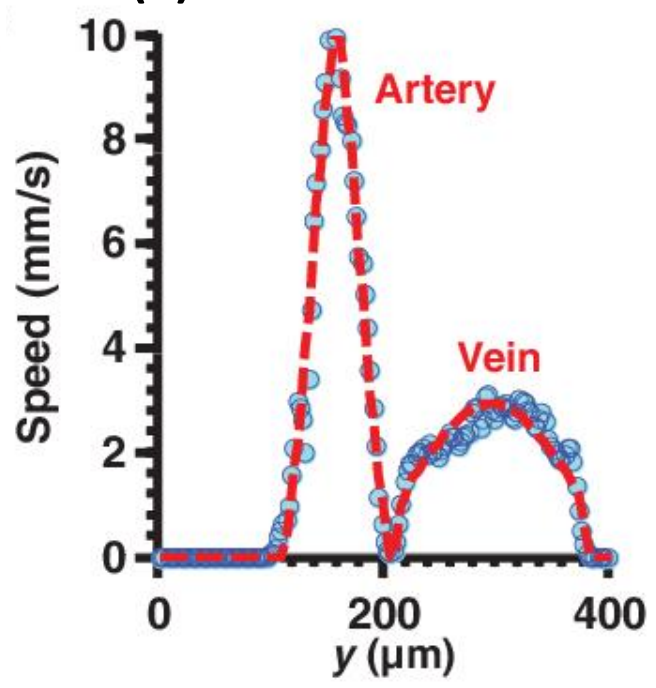

Figure 6-2: (a) Photoacoustic microscopy image of total hemoglobin concentration $\left(\mathrm{C}_{\mathrm{Hb}}\right)$ from the skin of a mouse. Scale bar denotes $500 \mu \mathrm{m}$. (b) $\mathrm{sO}_{2}$ map of the area indicated by the dashed box in the hemoglobin image. (c) PA flow map of dashed box area shown in (b). The pseudo colors indicate the direction of blood flow relative to the scanning direction. Scale bar denotes $125 \mu \mathrm{m}$. (d) Profile of blood flow speed across the dashed line in (c) showing the parabolic flow profiles expected from arteries and veins. Adapted from reference [326]. 


\section{CHAPTER 6}

\subsubsection{Imaging the extracellular matrix of tumors}

The tumor microenvironment contains a complex collection of cancer cells (malignant or nonmalignant), blood and lymphatic vessels which are all embedded in a fibrous material known as the extracellular matrix [54]. The matrix consists of interlocked meshes of water, minerals, proteoglycans and fibrous proteins, all of which are secreted by the very same cells that reside within the scaffold. The most abundant matrix protein polymers are collagens. Along other matrix constituents, collagen provides both structural support to the cellular components of the tumor and plays a significant biochemical supporting role [327]. Figure 6-3 summarizes the wide-ranging influences of collagen in cancer cell behavior. These effects arise since the collagen scaffolds compress blood and lymphatic vessels, causing a solid stress to many parts of the tumor. This stress, in turn, can reduce or even halt blood flow. The latter is caused from the lack of lymphatic drainage, leading to interstitial fluid buildup [179]. The increased interstitial fluid pressure negatively impairs drug delivery and compromises the ability of blood vessels to distribute oxygen and tumor-fighting immune cells. This also fosters hypoxic conditions which in turn can spur malignant and even normal cells to suppress the activity of immune anti-cancer regulators [328]. Moreover, the hypoxic conditions of the tumor increase the invasive tendencies of cancer cells to travel away from the primary tumor, leading to metastasis. Depletion of the extracellular matrix has been shown to improve survival rates by decompressing blood vessels [329], [330]. As a result, the solid stress in tumors decreases, thus increasing vascular perfusion, which in turn potentiates chemotherapy and reduces hypoxia [328].

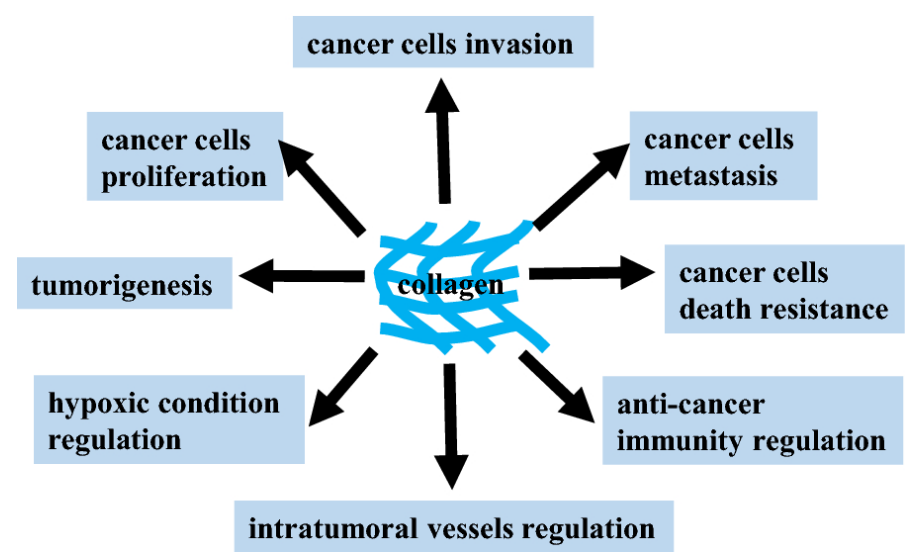

Figure 6-3: The contributions of collagen to the biological activities of cancer cells. Adapted from reference [331]. 


\section{CHAPTER 6}

Collagen is also an endogenous optical chromophore and has been previously quantified in liver disease [332], intestinal fibrosis [333] or muscular dystrophy [334] using PA imaging. These studies all take advantage of the increased optical absorption of collagen in wavelengths higher than $1000 \mathrm{~nm}$. However, water also efficiently absorbs light at the same wavelengths. This limits the penetration depth and the practical utility of the approach given the high-water content within tissue and the common use of water-based ultrasound coupling gels. Unrelated to the work of my dissertation, I have developed an approach that quantifies the spatial distribution and concentration of collagen at shorter wavelengths, where light can penetrate more efficiently inside tissue. This technique is applied to the quantification of the fibrosis burden of kidney transplants and it is currently under review in the Journal of Clinical Investigations Insights. As shown in Figure 6-4, this quantification approach can accurately unmix the concentration of collagen in the presence of blood, as is the case with tumors. Additionally, this algorithm can quantify the concentration of collagen in mouse kidneys when various degrees of fibrosis are induced (Figure 6-4d).

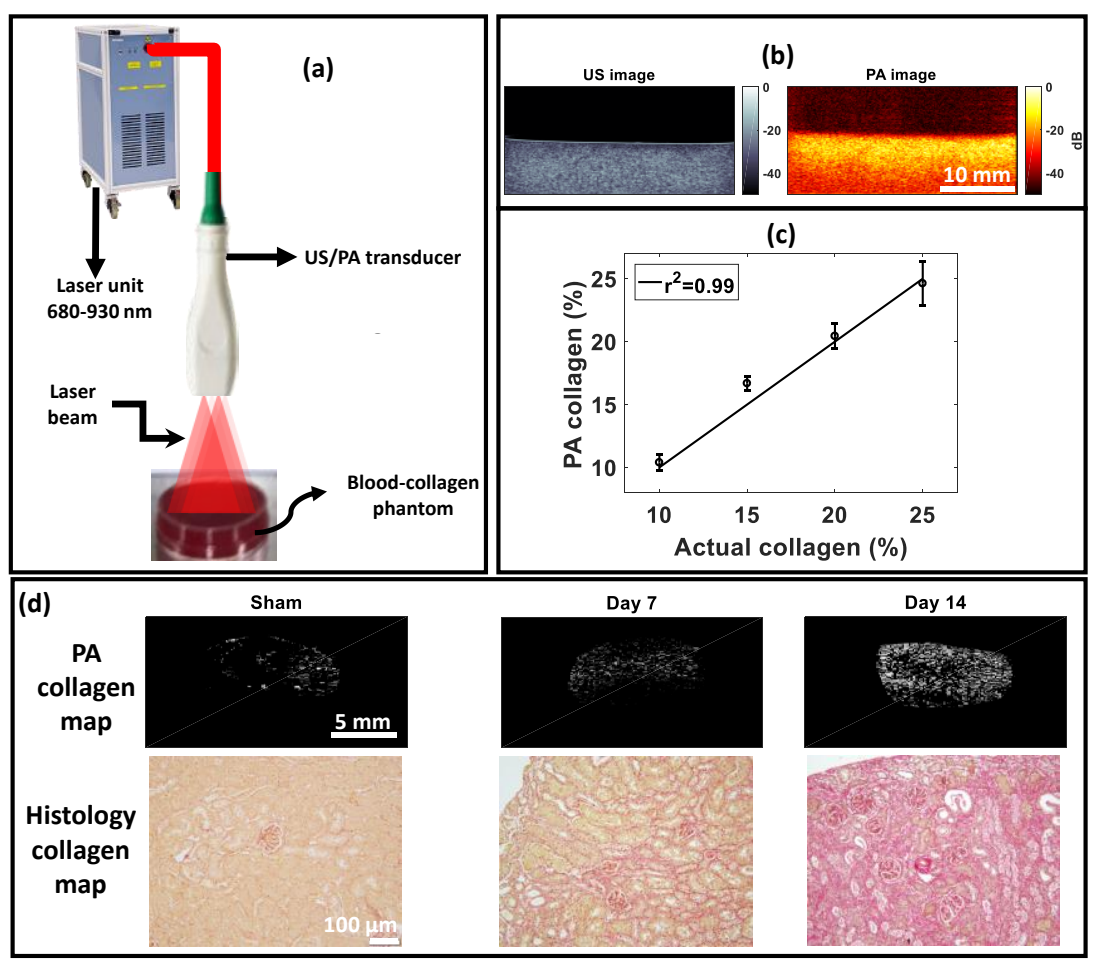

Figure 6-4: (a) PA imaging setup for blood collagen phantom gels. (b) Representative co-registered US and PA images (at $850 \mathrm{~nm}$ ) of a blood collagen phantom. (c) Validation of the unique spectral unmixing algorithm to quantify collagen. Error bars represent the standard deviation of the mean, with 60 measurements per phantom. The $r^{2}$ denotes the goodness of the linear fit. (d) Representative PA and histology collagen maps of mouse kidneys with progressively higher degrees of fibrosis at day 7 and day 14 compare to sham. 


\section{CHAPTER 6}

These results suggest that collagen can be quantified using PA imaging at clinically relevant optical illumination wavelengths $(680-930 \mathrm{~nm})$. This can be done non-invasively using a portable PA transducer (Figure 6-4a), thus facilitating the clinical translation of the approach. Given the role that collagen plays in the development of cancer and the attempts at managing hypoxia by alleviating the collagen-induced tumor solid stress [54], it might be possible to monitor tumor collagen content using PA imaging. This would provide a new cancer treatment PA biomarker that can be used to monitor the changes in extracellular matrix, expanding the vascular capabilities of this modality.

\subsubsection{Strengthening the predictive power of PA biomarkers}

This thesis shows the theoretical and experimental development of PA biomarkers that can be used for cancer treatment monitoring. In most preclinical and clinical PA imaging systems available, US and PA data are detected by the same transducer and therefore, inherently co-registered [82], [93], [279]. This permits simultaneous acquisitions of both anatomical and functional information which is available from US and PA, respectively. In the context of cancer treatment monitoring, Czarnota and colleagues have demonstrated that US imaging biomarkers can detect the response of primary tumors early after the start of neoadjuvant chemotherapy [24], [169], [232]. This approach can provide response classification as early as one week post-treatment with $78 \%$ accuracy when QUS, texture and molecular features of the tumor are combined [335]. The predictive accuracy reaches a maximum value of $86 \%$ at 4 weeks after the initiation of treatment. Moreover, Taddayyon et al explored the potential of using pre-treatment textural features of QUS parametric images of breast tumors and their margin zones to predict chemotherapy responsiveness [336], [337]. Figure 6-5 shows how textural analysis of the tumor core and margins before treatment can be used differentiate treatment responder from non-responders by quantifying the infiltration of the primary tumor into the surrounding normal tissue. 


\section{CHAPTER 6}

(a)

(b)

(c)

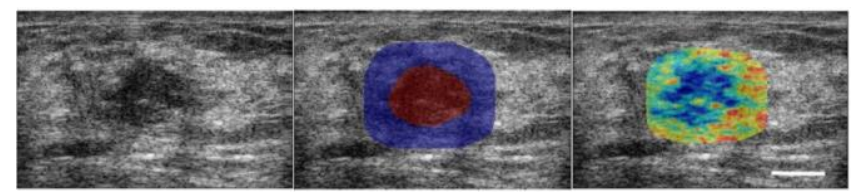

(d)

(e)

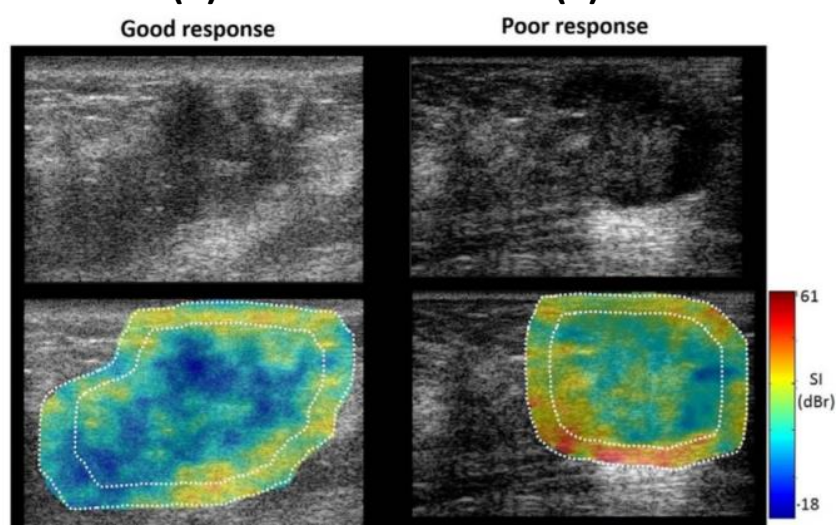

Figure 6-5: (a) Representative US B-mode of a locally advanced breast cancer tumor and (b) its corresponding ROI for the tumor core (red) and a $5 \mathrm{~mm}$ margin thickness (blue). (c) The corresponding spectral intercept (SI) parametric image which can differentiate (d) good vs. (e) poor treatment response. Scale bar denotes $1 \mathrm{~cm}$. Adapted from reference [336].

These encouraging results have the potential to transform cancer treatment monitoring. PA imaging can contribute further to this quest, especially given the fact that it shares the presence of image speckle with the ultrasonic counterpart. This opens the possibility for performing textural analysis on multiparametric images of PA biomarkers, both on the functional (oxygenation, hemoglobin, oxy and deoxy) and structural (frequency-based parameters such as spectral slope, midband fit, intercept, cepstral spacing) types derived in Chapter 2. Moreover, since these parameters were shown to change early post-treatment, it might be beneficial to combine this analysis with the US structural biomarkers at those early timepoints. In particular, the tumor rimcore textural analysis in QUS allowed for a deeper understanding of the tumor microenvironment and the changes it undergoes through treatment. PA can contribute further information about the level of hypoxia (by estimating the $\mathrm{sO}_{2}$ ), extracellular stiffness (by estimating the collagen matrix) or the degree of vascular damage (as shown in this thesis). Performing this analysis before the treatment commences can potentially lead to substantial savings in the cost and resources required to deliver cancer therapies which turn out to be ineffective. The proposed work offers a tool for oncologists to quantify the clinical decision making in hopes of improving cancer treatment outcomes. 


\title{
APPENDIX
}

\section{A.1 Permissions to reproduce copyrighted material}

\section{A.1.1 Chapters 2 and 4 permissions from Elsevier}

\author{
1/3/2020 Gmail - Re: Request to use article content in a PhD thesis [200102-006839] \\ Reply-To: Permissions Helpdesk <permissionshelpdesk@elsevier.com> \\ To: eno.hysi@ryerson.ca
}

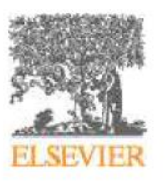

Dear Eno Hysi,

Thank you for your query.

Please note that, as one of the authors of this article, you retain the right to reuse it in your thesis/dissertation. You do not require formal permission to do so. You are permitted to post this Elsevier article online if it is embedded within your thesis. You are also permitted to post your Author Accepted Manuscript online.

However posting of the final published article is prohibited.

"As per our Sharing Policy, authors are permitted to post the Accepted version of their article on their institutional repository - as long as it is for internal institutional use only.

It can only be shared publicly on that site once the journal-specific embargo period has lapsed. For a list of embargo periods please see: Embargo List.

You are not permitted to post the Published Journal Article (PJA) on the repository."

Please feel free to contact me if you have any queries.

Regards,

Kaveri

Permissions Helpdesk

ELSEVIER |Operations

From: Administrator

Date: 02/01/2020 05.37 AM

Dear Customer

Thanks you for contacting Elsevier's Permissions Helpdesk.

This is an automated acknowledgement to confirm we have received your query. Ticket number 200102-006839 has been opened on your behalf and we aim to respond within seven business days.

Regards,

Permissions Helpdesk 


\section{A.1.2 Chapter 3 permission from PLOS}

Licenses and Copyright

The following policy applies to all PLOS journals, unless otherwise noted.

\section{Reuse of PLOS Article Content}

PLOS applies the Creative Commons Attribution (CC BY) license to articles and other works we publish. If you submit your pap publication by PLOS, you agree to have the CC BY license applied to your work. Under this Open Access license, you as the at that anyone can reuse your article in whole or part for any purpose, for free, even for commercial purposes. Anyone may copy, $c$ or reuse the content as long as the author and original source are properly cited. This facilitates freedom in re-use and also ens PLOS content can be mined without barriers for the needs of research.

\section{Content Owned by Someone Else}

If you have written permission to do so, yes. If your manuscript contains content such as photos, images, figures, tables, audio $f$ videos, etc., that you or your co-authors do not own, we will require you to provide us with proof that the owner of that content (a given you written permission to use it, and (b) has approved of the CC BY license being applied to their content. We provide a fs can use to ask for and obtain permission from the owner. Download the form (PDF).

(1) If you do not have owner permission, we will ask you to remove that content and/or replace it with other content that you own or have such permissio

Don't assume that you can use any content you find on the Internet, or that the content is fair game just because it isn't clear wh owner is or what license applies. It's up to you to ascertain what rights you have-if any-to use that content.

\section{Using Article Content Previously Published in Another Journal}

Many authors assume that if they previously published a paper through another publisher, they own the rights to that content an freely use that content in their PLOS paper, but that's not necessarily the case - it depends on the license that covers the other Some publishers allow free and unrestricted re-use of article content they own, such as under the CC BY license. Other publish licenses that allow re-use only if the same license is applied by the person or publisher re-using the content.

If the paper was published under a CC BY license or another license that allows free and unrestricted use, you may use the con your PLOS paper provided that you give proper attribution, as explained above.

If the content was published under a more restrictive license, you must ascertain what rights you have under that license. At a $n$ review the license to make sure you can use the content. Contact that publisher if you have any questions about the license terr staff cannot give you legal advice about your rights to use third-party content. If the license does not permit you to use the conte paper that will be covered by an unrestricted license, you must obtain written permission from the publisher to use the content ir PLOS paper. Please do not include any content in your PLOS paper which you do not have rights to use, and always give props attribution.

\section{Acceptable Licenses for Data Repositories}

If any relevant accompanying data is submitted to repositories with stated licensing policies, the policies should not be more res than CC BY.

\section{Removal of Content Used Without Clear Rights}

PLOS reserves the right to remove any photos, captures, images, figures, tables, illustrations, audio and video files, and the like paper, whether before or after publication, if we have reason to believe that the content was included in your paper without perm from the owner of the content.

\section{Guidelines for Trademarks}

Ensure that any reference to a trademark (such as a brand name) is used as an adjective, and not a noun or verb. The tradema be immediately followed by the generic term for the object that it modifies. Note that because a trademark cannot be used as a । cannot be presented in the possessive or plural form. Please see the following example for reference:

INCORRECT: The stimuli were presented on 12 MacBook Pros $₫$.

CORRECT: The stimuli were presented on 12 Macbook Pro® computers.

Giving Proper Attribution for Use of Content 
When citing a PLOS research article, use the "Vancouver style", as outlined in our Submission Guidelines. For example:

Kaltenbach LS et al. (2007) Huntingtin Interacting Proteins Are Genetic Modifiers of Neurodegeneration. PLOS Genet 3(5): e82. do: 10.1371 /journal.pgen.0030082.

When citing non-article content from a PLOS website (e.g., blog content), provide a link to the content, and cite the title and autr that content.

For examples of proper attribution to other types of content, see websites such as Open.Michigan.

Give Feedback

\begin{tabular}{|c|c|c|c|c|}
\hline \multicolumn{5}{|c|}{ How helpful was the information on this page? } \\
\hline Not at all helpful & Not very helpful & Helpful & Very helpful & Extremely helpful \\
\hline 0 & 0 & 0 & 0 & 0 \\
\hline \multicolumn{5}{|c|}{ Send feedback } \\
\hline
\end{tabular}




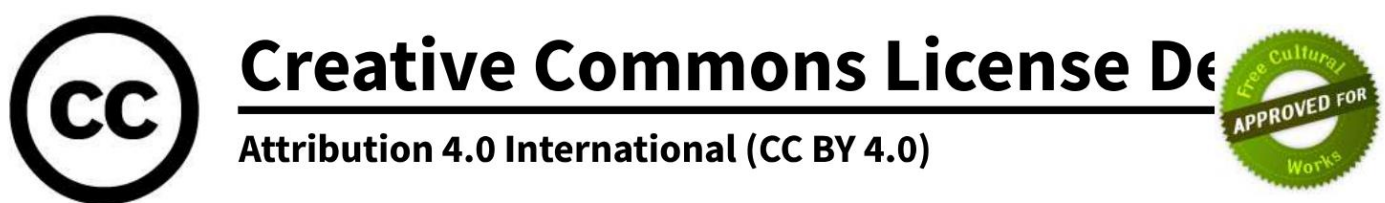

This is a human-readable summary of (and not a substitute for) the license.

\section{You are free to:}

Share - copy and redistribute the material in any medium or format

Adapt - remix, transform, and build upon the material

for any purpose, even commercially.

The licensor cannot revoke these freedoms as long as you follow the license terms.

\section{Under the following terms:}

Attribution - You must give appropriate credit, provide a link to the license, and indicate if changes were made. You may do so in any reasonable manner, but not in any way that suggests the licensor endorses you or your use.

No additional restrictions - You may not apply legal terms or technological measures that legally restrict others from doing anything the license permits.

\section{Notices:}

You do not have to comply with the license for elements of the material in the public domain or where your use is permitted by an applicable exception or limitation.

No warranties are given. The license may not give you all of the permissions necessary for your intended use. For example, other rights such as publicity, privacy, or moral rights may limit how you use the material. 


\section{REFERENCES}

[1] S. B. Edge and C. C. Compton, "The American Joint Committee on Cancer: the 7th Edition of the AJCC Cancer Staging Manual and the Future of TNM," Ann. Surg. Oncol., vol. 17, no. 6, pp. 1471-1474, Jun. 2010, doi: 10.1245/s10434-010-0985-4.

[2] D. Cross and J. K. Burmester, "Gene therapy for cancer treatment: past, present and future," Clin. Med. Res., vol. 4, no. 3, pp. 218-227, 2006.

[3] "Breast cancer statistics - Canadian Cancer Society," www.cancer.ca. http://www.cancer.ca/en/cancer-information/cancer-type/breast/statistics/?region=on (accessed Apr. 27, 2016).

[4] V. B. Shahinian, Y.-F. Kuo, J. L. Freeman, and J. S. Goodwin, "Risk of fracture after androgen deprivation for prostate cancer," N. Engl. J. Med., vol. 352, no. 2, pp. 154-164, Jan. 2005, doi: 10.1056/NEJMoa041943.

[5] B. D. Smith, G. L. Smith, A. Hurria, G. N. Hortobagyi, and T. A. Buchholz, "Future of Cancer Incidence in the United States: Burdens Upon an Aging, Changing Nation," J. Clin. Oncol., vol. 27 , no. 17, pp. 2758-2765, Jun. 2009, doi: 10.1200/JCO.2008.20.8983.

[6] J. H. Thrall, "Personalized Medicine1," Radiology, vol. 231, no. 3, pp. 613-616, Jun. 2004, doi: $10.1148 /$ radiol.2313040323.

[7] J. J. Smith, A. G. Sorensen, and J. H. Thrall, "Biomarkers in Imaging: Realizing Radiology's Future," Radiology, vol. 227, no. 3, pp. 633-638, Jun. 2003, doi: 10.1148/radiol.2273020518.

[8] "Precision Medicine Initiative," National Institutes of Health (NIH), 2015. https://www.nih.gov/precision-medicine-initiative-cohort-program (accessed Apr. 27, 2016).

[9] R. K. Jain, "Normalizing Tumor Microenvironment to Treat Cancer: Bench to Bedside to Biomarkers," J. Clin. Oncol., vol. 31, no. 17, pp. 2205-2218, Jun. 2013, doi: 10.1200/JCO.2012.46.3653.

[10] A. Sadeghi-Naini et al., "Imaging innovations for cancer therapy response monitoring," Imaging Med., vol. 4, no. 3, pp. 311-327, Jun. 2012, doi: 10.2217/iim.12.23.

[11] P. Therasse et al., "New guidelines to evaluate the response to treatment in solid tumors. European Organization for Research and Treatment of Cancer, National Cancer Institute of the United States, National Cancer Institute of Canada," J. Natl. Cancer Inst., vol. 92, no. 3, pp. 205-216, Feb. 2000.

[12] S. Litière, S. Collette, E. G. E. de Vries, L. Seymour, and J. Bogaerts, "RECIST — learning from the past to build the future," Nat. Rev. Clin. Oncol., vol. 14, no. 3, pp. 187-192, Mar. 2017, doi: 10.1038/nrclinonc.2016.195.

[13] K. Brindle, "New approaches for imaging tumour responses to treatment," Nat. Rev. Cancer, vol. 8, no. 2, pp. 94-107, Feb. 2008, doi: 10.1038/nrc2289.

[14] L. C. Michaelis and M. J. Ratain, "Measuring response in a post-RECIST world: from black and white to shades of grey," Nat. Rev. Cancer, vol. 6, no. 5, pp. 409-414, May 2006, doi: $10.1038 / \mathrm{nrc} 1883$.

[15] G. K. von Schulthess, H. C. Steinert, and T. F. Hany, "Integrated PET/CT: current applications and future directions," Radiology, vol. 238, no. 2, pp. 405-422, Feb. 2006, doi: 10.1148/radiol.2382041977.

[16] S. Stroobants et al., "18FDG-Positron emission tomography for the early prediction of response in advanced soft tissue sarcoma treated with imatinib mesylate (Glivec)," Eur. J. Cancer Oxf. Engl. 1990, vol. 39, no. 14, pp. 2012-2020, Sep. 2003. 
[17] C. Bailey, K. L. Desmond, G. J. Czarnota, and G. J. Stanisz, "Quantitative magnetization transfer studies of apoptotic cell death," Magn. Reson. Med., vol. 66, no. 1, pp. 264-269, Jul. 2011, doi: $10.1002 / \mathrm{mrm} .22820$.

[18] M. O. Leach et al., "The assessment of antiangiogenic and antivascular therapies in earlystage clinical trials using magnetic resonance imaging: issues and recommendations," $\mathrm{Br} . \mathrm{J}$. Cancer, vol. 92, no. 9, pp. 1599-1610, May 2005, doi: 10.1038/sj.bjc.6602550.

[19] M. Lamuraglia et al., "Clinical relevance of contrast-enhanced ultrasound in monitoring antiangiogenic therapy of cancer: current status and perspectives," Crit. Rev. Oncol. Hematol., vol. 73, no. 3, pp. 202-212, Mar. 2010, doi: 10.1016/j.critrevonc.2009.06.001.

[20] "Biomarkers and surrogate endpoints: Preferred definitions and conceptual framework," Clin. Pharmacol. Ther., vol. 69, no. 3, pp. 89-95, 2001, doi: 10.1067/mcp.2001.113989.

[21] FDA-NIH Biomarker Working Group, BEST (Biomarkers, EndpointS, and other Tools) Resource. Silver Spring (MD): Food and Drug Administration (US), 2016.

[22] P. Rolan, "The contribution of clinical pharmacology surrogates and models to drug development--a critical appraisal," Br. J. Clin. Pharmacol., vol. 44, no. 3, pp. 219-225, Sep. 1997, doi: 10.1046/j.1365-2125.1997.t01-1-00583.x.

[23] J. P. B. O'Connor et al., "Imaging biomarker roadmap for cancer studies," Nat. Rev. Clin. Oncol., vol. 14, no. 3, pp. 169-186, Mar. 2017, doi: 10.1038/nrclinonc.2016.162.

[24] A. Sadeghi-Naini et al., "Quantitative ultrasound evaluation of tumor cell death response in locally advanced breast cancer patients receiving chemotherapy," Clin. Cancer Res. Off. J. Am. Assoc. Cancer Res., vol. 19, no. 8, pp. 2163-2174, Apr. 2013, doi: 10.1158/10780432.CCR-12-2965.

[25] R. J. Hicks, "The role of PET in monitoring therapy," Cancer Imaging, vol. 5, no. 1, pp. 5157, Jun. 2005, doi: 10.1102/1470-7330.2005.0006.

[26] A. Cerussi et al., "Predicting response to breast cancer neoadjuvant chemotherapy using diffuse optical spectroscopy," Proc. Natl. Acad. Sci. U. S. A., vol. 104, no. 10, pp. 40144019, Mar. 2007, doi: 10.1073/pnas.0611058104.

[27] F. Lizzi, L. Katz, L. St. Louis, and D. J. Coleman, "Applications of spectral analysis in medical ultrasonography," Ultrasonics, vol. 14, no. 2, pp. 77-80, Mar. 1976, doi: 10.1016/0041-624X(76)90103-7.

[28] F. L. Lizzi, M. Greenebaum, E. J. Feleppa, M. Elbaum, and D. J. Coleman, "Theoretical framework for spectrum analysis in ultrasonic tissue characterization," J. Acoust. Soc. Am., vol. 73, no. 4, pp. 1366-1373, Apr. 1983.

[29] F. L. Lizzi, M. Ostromogilsky, E. J. Feleppa, M. C. Rorke, and M. M. Yaremko, "Relationship of Ultrasonic Spectral Parameters to Features of Tissue Microstructure," IEEE Trans. Ultrason. Ferroelectr. Freq. Control, vol. 34, no. 3, pp. 319-329, May 1987, doi: 10.1109/T-UFFC.1987.26950.

[30] J. Mamou and M. L. Oelze, Eds., Quantitative Ultrasound in Soft Tissues. Dordrecht: Springer Netherlands, 2013.

[31] L. A. Wirtzfeld et al., "Cross-imaging platform comparison of ultrasonic backscatter coefficient measurements of live rat tumors," J. Ultrasound Med. Off. J. Am. Inst. Ultrasound Med., vol. 29, no. 7, pp. 1117-1123, Jul. 2010.

[32] G. J. Czarnota et al., "Ultrasonic biomicroscopy of viable, dead and apoptotic cells," Ultrasound Med. Biol., vol. 23, no. 6, pp. 961-965, 1997. 
[33] G. J. Czarnota et al., "Ultrasound imaging of apoptosis: high-resolution non-invasive monitoring of programmed cell death in vitro, in situ and in vivo," Br. J. Cancer, vol. 81, no. 3, pp. 520-527, Oct. 1999, doi: 10.1038/sj.bjc.6690724.

[34] M. C. Kolios, G. J. Czarnota, M. Lee, J. W. Hunt, and M. D. Sherar, "Ultrasonic spectral parameter characterization of apoptosis," Ultrasound Med. Biol., vol. 28, no. 5, pp. 589-597, May 2002.

[35] A. Sadeghi-Naini et al., "Quantitative ultrasound spectroscopic imaging for characterization of disease extent in prostate cancer patients," Transl. Oncol., vol. 8, no. 1, pp. 25-34, Feb. 2015, doi: 10.1016/j.tranon.2014.11.005.

[36] R. Weissleder and U. Mahmood, "Molecular imaging," Radiology, vol. 219, no. 2, pp. 316333, May 2001, doi: 10.1148/radiology.219.2.r01ma19316.

[37] F. D. S. E. Melo, L. Vermeulen, E. Fessler, and J. P. Medema, "Cancer heterogeneity-a multifaceted view," EMBO Rep., vol. 14, no. 8, pp. 686-695, Aug. 2013, doi: 10.1038/embor.2013.92.

[38] V. Ntziachristos and B. Chance, "Probing physiology and molecular function using optical imaging: applications to breast cancer," Breast Cancer Res. BCR, vol. 3, no. 1, pp. 41-46, 2001.

[39] D. R. Leff et al., "Diffuse optical imaging of the healthy and diseased breast: a systematic review," Breast Cancer Res. Treat., vol. 108, no. 1, pp. 9-22, Mar. 2008, doi: 10.1007/s10549-007-9582-z.

[40] T. Vo-Dinh, Biomedical Photonics Handbook. CRC Press, 2003.

[41] D. A. Boas, A. M. Dale, and M. A. Franceschini, "Diffuse optical imaging of brain activation: approaches to optimizing image sensitivity, resolution, and accuracy," NeuroImage, vol. 23 Suppl 1, pp. S275-288, 2004, doi: 10.1016/j.neuroimage.2004.07.011.

[42] H. Soliman et al., "Functional imaging using diffuse optical spectroscopy of neoadjuvant chemotherapy response in women with locally advanced breast cancer," Clin. Cancer Res. Off. J. Am. Assoc. Cancer Res., vol. 16, no. 9, pp. 2605-2614, May 2010, doi: 10.1158/10780432.CCR-09-1510.

[43] D. Roblyer et al., "Optical imaging of breast cancer oxyhemoglobin flare correlates with neoadjuvant chemotherapy response one day after starting treatment," Proc. Natl. Acad. Sci. U. S. A., vol. 108, no. 35, pp. 14626-14631, Aug. 2011, doi: 10.1073/pnas.1013103108.

[44] W. T. Tran et al., "Multiparametric monitoring of chemotherapy treatment response in locally advanced breast cancer using quantitative ultrasound and diffuse optical spectroscopy," Oncotarget, Mar. 2016, doi: 10.18632/oncotarget.7844.

[45] J. P. Culver, V. Ntziachristos, M. J. Holboke, and A. G. Yodh, "Optimization of optode arrangements for diffuse optical tomography: A singular-value analysis," Opt. Lett., vol. 26, no. 10, pp. 701-703, May 2001, doi: 10.1364/OL.26.000701.

[46] R. K. Wang and V. V. Tuchin, Advanced Biophotonics: Tissue Optical Sectioning. Taylor \& Francis, 2016.

[47] P. Vaupel and M. Hockel, "Blood supply, oxygenation status and metabolic micromilieu of breast cancers: characterization and therapeutic relevance," Int. J. Oncol., vol. 17, no. 5, pp. 869-879, Nov. 2000, doi: 10.3892/ijo.17.5.869.

[48] H. J. Feldmann, "Oxygenation of human tumors - implications for combined therapy," Lung Cancer, vol. 33, pp. S77-S83, Oct. 2001, doi: 10.1016/S0169-5002(01)00306-3.

[49] J. Folkman, “Tumor Angiogenesis Factor," Cancer Res., vol. 34, no. 8, pp. 2109-2113, Aug. 1974. 
[50] J. Folkman, "Fighting cancer by attacking its blood supply," Sci. Am., vol. 275, no. 3, pp. 150-154, Sep. 1996.

[51] P. Carmeliet and R. K. Jain, "Angiogenesis in cancer and other diseases," Nature, vol. 407, no. 6801, pp. 249-257, Sep. 2000, doi: 10.1038/35025220.

[52] B. J. Vakoc et al., "Three-dimensional microscopy of the tumor microenvironment in vivo using optical frequency domain imaging," Nat. Med., vol. 15, no. 10, pp. 1219-1223, Oct. 2009, doi: 10.1038/nm.1971.

[53] R. K. Jain, "Barriers to drug delivery in solid tumors," Sci. Am., vol. 271, no. 1, pp. 58-65, Jul. 1994.

[54] R. K. Jain, “An Indirect Way to Tame Cancer,” Sci. Am., vol. 310, no. 2, pp. 46-53, 2014.

[55] W. H. AlMalki, I. Shahid, A. Y. Mehdi, and M. H. Hafeez, "Assessment methods for angiogenesis and current approaches for its quantification," Indian J. Pharmacol., vol. 46, no. 3, pp. 251-256, 2014, doi: 10.4103/0253-7613.132152.

[56] R. K. Jain, “Taming vessels to treat cancer," Sci. Am., vol. 298, no. 1, pp. 56-63, Jan. 2008, doi: 10.1038/scientificamerican0108-56.

[57] M. Molls, P. Stadler, A. Becker, H. J. Feldmann, and J. Dunst, "Relevance of oxygen in radiation oncology. Mechanisms of action, correlation to low hemoglobin levels," Strahlenther. Onkol. Organ Dtsch. Rontgengesellschaft Al, vol. 174 Suppl 4, pp. 13-16, Dec. 1998.

[58] J.-T. Chi et al., "Gene Expression Programs in Response to Hypoxia: Cell Type Specificity and Prognostic Significance in Human Cancers," PLOS Med., vol. 3, no. 3, p. e47, Jan. 2006, doi: 10.1371/journal.pmed.0030047.

[59] B. A. Teicher, "Physiologic mechanisms of therapeutic resistance. Blood flow and hypoxia," Hematol. Oncol. Clin. North Am., vol. 9, no. 2, pp. 475-506, Apr. 1995.

[60] P. Okunieff, B. Fenton, and Y. Chen, "Past, present, and future of oxygen in cancer research," Adv. Exp. Med. Biol., vol. 566, pp. 213-222, 2005, doi: 10.1007/0-387-26206-7_29.

[61] M. Höckel and P. Vaupel, "Biological consequences of tumor hypoxia," Semin. Oncol., vol. 28, no. 2 Suppl 8, pp. 36-41, Apr. 2001.

[62] M. R. Horsman, L. S. Mortensen, J. B. Petersen, M. Busk, and J. Overgaard, "Imaging hypoxia to improve radiotherapy outcome," Nat. Rev. Clin. Oncol., vol. 9, no. 12, pp. 674687, Dec. 2012, doi: 10.1038/nrclinonc.2012.171.

[63] R. K. Jain and P. F. Carmeliet, "Vessels of death or life," Sci. Am., vol. 285, no. 6, pp. 3845, Dec. 2001.

[64] J. Folkman, "Fundamental concepts of the angiogenic process," Curr. Mol. Med., vol. 3, no. 7, pp. 643-651, 2003.

[65] V. P. Chauhan et al., "Normalization of tumour blood vessels improves the delivery of nanomedicines in a size-dependent manner," Nat. Nanotechnol., vol. 7, no. 6, pp. 383-388, Apr. 2012, doi: 10.1038/nnano.2012.45.

[66] R. K. Jain, "Delivery of molecular and cellular medicine to solid tumors," Adv. Drug Deliv. Rev., vol. 64, pp. 353-365, Dec. 2012, doi: 10.1016/j.addr.2012.09.011.

[67] A. Rapisarda and G. Melillo, "Overcoming disappointing results with antiangiogenic therapy by targeting hypoxia," Nat. Rev. Clin. Oncol., vol. 9, no. 7, pp. 378-390, Jul. 2012, doi: 10.1038/nrclinonc.2012.64.

[68] G. Jiménez-Valerio et al., "Resistance to Antiangiogenic Therapies by Metabolic Symbiosis in Renal Cell Carcinoma PDX Models and Patients," Cell Rep., vol. 15, no. 6, pp. 11341143, 10 2016, doi: 10.1016/j.celrep.2016.04.015. 
[69] G. Bergers and D. Hanahan, "Modes of resistance to anti-angiogenic therapy," Nat. Rev. Cancer, vol. 8, no. 8, pp. 592-603, Aug. 2008, doi: 10.1038/nrc2442.

[70] Y. Liu et al., "Carbon Ion Radiation Inhibits Glioma and Endothelial Cell Migration Induced by Secreted VEGF," PLOS ONE, vol. 9, no. 6, p. e98448, Jun. 2014, doi: 10.1371/journal.pone.0098448.

[71] H. J. Park, R. J. Griffin, S. Hui, S. H. Levitt, and C. W. Song, "Radiation-induced vascular damage in tumors: implications of vascular damage in ablative hypofractionated radiotherapy (SBRT and SRS)," Radiat. Res., vol. 177, no. 3, pp. 311-327, Mar. 2012, doi: $10.1667 / \mathrm{rr} 2773.1$.

[72] A. Sams, "Histological changes in the larger blood vessels of the hind limb of the mouse after X-irradiation," Int. J. Radiat. Biol. Relat. Stud. Phys. Chem. Med., vol. 9, pp. 165-174, 1965, doi: 10.1080/09553006514550211.

[73] M. Garcia-Barros et al., "Tumor response to radiotherapy regulated by endothelial cell apoptosis," Science, vol. 300, no. 5622, pp. 1155-1159, May 2003, doi: 10.1126/science.1082504.

[74] D. B. Jakubowski et al., "Monitoring neoadjuvant chemotherapy in breast cancer using quantitative diffuse optical spectroscopy: a case study," J. Biomed. Opt., vol. 9, no. 1, pp. 230-238, Feb. 2004, doi: 10.1117/1.1629681.

[75] S. Ueda et al., "Baseline Tumor Oxygen Saturation Correlates with a Pathologic Complete Response in Breast Cancer Patients Undergoing Neoadjuvant Chemotherapy," Cancer Res., vol. 72, no. 17, pp. 4318-4328, Sep. 2012, doi: 10.1158/0008-5472.CAN-12-0056.

[76] H. S. Yazdi et al., "Mapping breast cancer blood flow index, composition, and metabolism in a human subject using combined diffuse optical spectroscopic imaging and diffuse correlation spectroscopy," J. Biomed. Opt., vol. 22, no. 4, p. 045003, Apr. 2017, doi: 10.1117/1.JBO.22.4.045003.

[77] C. Sessa, A. Guibal, G. D. Conte, and C. Rüegg, "Biomarkers of angiogenesis for the development of antiangiogenic therapies in oncology: tools or decorations?," Nat. Clin. Pract. Oncol., vol. 5, no. 7, pp. 378-391, Jul. 2008, doi: 10.1038/ncponc1150.

[78] A. G. Bell, "LXVIII. Upon the production of sound by radiant energy," Lond. Edinb. Dublin Philos. Mag. J. Sci., vol. 11, no. 71, pp. 510-528, Jan. 1881, doi: 10.1080/14786448108627053.

[79] A. Rosencwaig, Photoacoustics and Photoacoustic Spectroscopy, Volume 57 ed. edition. New York: John Wiley \& Sons Inc, 1981.

[80] S. Hu and L. V. Wang, "Optical-Resolution Photoacoustic Microscopy: Auscultation of Biological Systems at the Cellular Level," Biophys. J., vol. 105, no. 4, pp. 841-847, Aug. 2013, doi: 10.1016/j.bpj.2013.07.017.

[81] W. Choi, E.-Y. Park, S. Jeon, and C. Kim, "Clinical photoacoustic imaging platforms," Biomed. Eng. Lett., vol. 8, no. 2, pp. 139-155, Apr. 2018, doi: 10.1007/s13534-018-0062-7.

[82] A. Karlas et al., "Cardiovascular optoacoustics: From mice to men - A review," Photoacoustics, vol. 14, pp. 19-30, Jun. 2019, doi: 10.1016/j.pacs.2019.03.001.

[83] L. V. Wang and S. Hu, "Photoacoustic tomography: in vivo imaging from organelles to organs," Science, vol. 335, no. 6075, pp. 1458-1462, Mar. 2012, doi: 10.1126/science. 1216210.

[84] S. Fox, Human Physiology, 13 edition. New York, NY: McGraw-Hill Education, 2012. 
[85] S. Hu and L. V. Wang, "Photoacoustic imaging and characterization of the microvasculature," J. Biomed. Opt., vol. 15, no. 1, p. 011101, Feb. 2010, doi: 10.1117/1.3281673.

[86] A. B. E. Attia et al., "A review of clinical photoacoustic imaging: Current and future trends," Photoacoustics, vol. 16, p. 100144, Dec. 2019, doi: 10.1016/j.pacs.2019.100144.

[87] P. Beard, "Biomedical photoacoustic imaging," Interface Focus, vol. 1, no. 4, pp. 602-631, Aug. 2011, doi: 10.1098/rsfs.2011.0028.

[88] R. A. Kruger, R. B. Lam, D. R. Reinecke, S. P. Del Rio, and R. P. Doyle, "Photoacoustic angiography of the breast," Med. Phys., vol. 37, no. 11, pp. 6096-6100, Nov. 2010, doi: 10.1118/1.3497677.

[89] C. Kim, K. H. Song, F. Gao, and L. V. Wang, "Sentinel lymph nodes and lymphatic vessels: noninvasive dual-modality in vivo mapping by using indocyanine green in rats--volumetric spectroscopic photoacoustic imaging and planar fluorescence imaging," Radiology, vol. 255, no. 2, pp. 442-450, May 2010, doi: 10.1148/radiol.10090281.

[90] A. Danielli et al., "Label-free photoacoustic nanoscopy," J. Biomed. Opt., vol. 19, no. 8, p. 086006, Aug. 2014, doi: 10.1117/1.JBO.19.8.086006.

[91] S. Park, C. Lee, J. Kim, and C. Kim, "Acoustic resolution photoacoustic microscopy," Biomed. Eng. Lett., vol. 4, no. 3, pp. 213-222, Sep. 2014, doi: 10.1007/s13534-014-0153-z.

[92] L. G. Montilla, R. Olafsson, D. R. Bauer, and R. S. Witte, "Real-time photoacoustic and ultrasound imaging: a simple solution for clinical ultrasound systems with linear arrays," Phys. Med. Biol., vol. 58, no. 1, pp. N1-12, Jan. 2013, doi: 10.1088/0031-9155/58/1/N1.

[93] I. Steinberg, D. M. Huland, O. Vermesh, H. E. Frostig, W. S. Tummers, and S. S. Gambhir, "Photoacoustic clinical imaging," Photoacoustics, vol. 14, pp. 77-98, Jun. 2019, doi: 10.1016/j.pacs.2019.05.001.

[94] T. Vu, D. Razansky, and J. Yao, "Listening to tissues with new light: recent technological advances in photoacoustic imaging," J. Opt., 2019, doi: 10.1088/2040-8986/ab3b1a.

[95] E. Hysi, E. M. Strohm, and M. C. Kolios, "Probing Different Biological Length Scales Using Photoacoustics: From 1 To 1000 MHz," in Handbook of Photonics for Biomedical Engineering, A. H.-P. Ho, D. Kim, and M. G. Somekh, Eds. Springer Netherlands, 2014, pp. $1-18$.

[96] H. F. Zhang, K. Maslov, M. Sivaramakrishnan, G. Stoica, and L. V. Wang, "Imaging of hemoglobin oxygen saturation variations in single vessels in vivo using photoacoustic microscopy," Appl. Phys. Lett., vol. 90, no. 5, p. 053901, 2007, doi: 10.1063/1.2435697.

[97] L. Wang, K. Maslov, and L. V. Wang, "Single-cell label-free photoacoustic flowoxigraphy in vivo," Proc. Natl. Acad. Sci., vol. 110, no. 15, pp. 5759-5764, Apr. 2013, doi: 10.1073/pnas.1215578110.

[98] T. T. W. Wong et al., "Fast label-free multilayered histology-like imaging of human breast cancer by photoacoustic microscopy," Sci. Adv., vol. 3, no. 5, p. e1602168, May 2017, doi: 10.1126/sciadv.1602168.

[99] L. Li et al., "Single-impulse panoramic photoacoustic computed tomography of small-animal whole-body dynamics at high spatiotemporal resolution," Nat. Biomed. Eng., vol. 1, no. 5, pp. 1-11, May 2017, doi: 10.1038/s41551-017-0071.

[100] X. L. Deán-Ben, T. F. Fehm, S. J. Ford, S. Gottschalk, and D. Razansky, "Spiral volumetric optoacoustic tomography visualizes multi-scale dynamics in mice," Light Sci. Appl., vol. 6, no. 4, pp. e16247-e16247, Apr. 2017, doi: 10.1038/1sa.2016.247. 
[101] M. Nasiriavanaki, J. Xia, H. Wan, A. Q. Bauer, J. P. Culver, and L. V. Wang, "Highresolution photoacoustic tomography of resting-state functional connectivity in the mouse brain," Proc. Natl. Acad. Sci., vol. 111, no. 1, pp. 21-26, Jan. 2014, doi: 10.1073/pnas.1311868111.

[102] J. Brunker, J. Yao, J. Laufer, and S. E. Bohndiek, "Photoacoustic imaging using genetically encoded reporters: a review," J. Biomed. Opt., vol. 22, no. 7, 01 2017, doi: 10.1117/1.JBO.22.7.070901.

[103] J. Jo, C. H. Lee, R. Kopelman, and X. Wang, "In vivo quantitative imaging of tumor pH by nanosonophore assisted multispectral photoacoustic imaging," Nat. Commun., vol. 8, no. 1, p. 471, 07 2017, doi: 10.1038/s41467-017-00598-1.

[104] M. Heijblom et al., "Visualizing breast cancer using the Twente photoacoustic mammoscope: what do we learn from twelve new patient measurements?," Opt. Express, vol. 20, no. 11, pp. 11582-11597, 2012.

[105] T. Kitai et al., "Photoacoustic mammography: initial clinical results," Breast Cancer Tokyo Jpn., vol. 21, no. 2, pp. 146-153, Mar. 2014, doi: 10.1007/s12282-012-0363-0.

[106] E. I. Neuschler et al., "A Pivotal Study of Optoacoustic Imaging to Diagnose Benign and Malignant Breast Masses: A New Evaluation Tool for Radiologists," Radiology, p. 172228, Nov. 2017, doi: 10.1148/radiol.2017172228.

[107] E. I. Neuschler et al., "Downgrading and Upgrading Gray-Scale Ultrasound BI-RADS Categories of Benign and Malignant Masses With Optoacoustics: A Pilot Study," AJR Am. J. Roentgenol., vol. 211, no. 3, pp. 689-700, 2018, doi: 10.2214/AJR.17.18436.

[108] L. Lin et al., "Single-breath-hold photoacoustic computed tomography of the breast," Nat. Commun., vol. 9, no. 1, p. 2352, 15 2018, doi: 10.1038/s41467-018-04576-z.

[109] B. E. Dogan et al., "Optoacoustic Imaging and Gray-Scale US Features of Breast Cancers: Correlation with Molecular Subtypes," Radiology, vol. 292, no. 3, pp. 564-572, Sep. 2019, doi: 10.1148/radiol.2019182071.

[110] T. Jin, H. Guo, H. Jiang, B. Ke, and L. Xi, "Portable optical resolution photoacoustic microscopy (pORPAM) for human oral imaging," Opt. Lett., vol. 42, no. 21, pp. 4434-4437, Nov. 2017, doi: 10.1364/OL.42.004434.

[111] B. Zabihian et al., "In vivo dual-modality photoacoustic and optical coherence tomography imaging of human dermatological pathologies," Biomed. Opt. Express, vol. 6, no. 9, pp. 3163-3178, Sep. 2015, doi: 10.1364/BOE.6.003163.

[112] M. Yang et al., "Photoacoustic/ultrasound dual imaging of human thyroid cancers: an initial clinical study," Biomed. Opt. Express, vol. 8, no. 7, pp. 3449-3457, Jul. 2017, doi: 10.1364/BOE.8.003449.

[113] F. Knieling et al., "Multispectral Optoacoustic Tomography for Assessment of Crohn's Disease Activity," N. Engl. J. Med., vol. 376, no. 13, pp. 1292-1294, 2017.

[114] I. Ivankovic, E. Merčep, C.-G. Schmedt, X. L. Deán-Ben, and D. Razansky, "Real-time Volumetric Assessment of the Human Carotid Artery: Handheld Multispectral Optoacoustic Tomography," Radiology, vol. 291, no. 1, pp. 45-50, Feb. 2019, doi: 10.1148/radiol.2019181325.

[115] I. Stoffels et al., "Metastatic status of sentinel lymph nodes in melanoma determined noninvasively with multispectral optoacoustic imaging," Sci. Transl. Med., vol. 7, no. 317, pp. 317ra199-317ra199, Dec. 2015, doi: 10.1126/scitranslmed.aad1278.

[116] M. A. A. Caballero, A. Rosenthal, A. Buehler, D. Razansky, and V. Ntziachristos, "Optoacoustic determination of spatio- temporal responses of ultrasound sensors," IEEE 
Trans. Ultrason. Ferroelectr. Freq. Control, vol. 60, no. 6, pp. 1234-1244, Jun. 2013, doi: 10.1109/TUFFC.2013.2687.

[117] C. Lutzweiler and D. Razansky, "Optoacoustic Imaging and Tomography: Reconstruction Approaches and Outstanding Challenges in Image Performance and Quantification," Sensors, vol. 13 , no. 6, pp. 7345-7384, Jun. 2013, doi: 10.3390/s130607345.

[118] M. Xu and L. V. Wang, "Universal back-projection algorithm for photoacoustic computed tomography," Phys. Rev. E Stat. Nonlin. Soft Matter Phys., vol. 71, no. 1 Pt 2, p. 016706, Jan. 2005, doi: 10.1103/PhysRevE.71.016706.

[119] G. Paltauf, J. A. Viator, S. A. Prahl, and S. L. Jacques, "Iterative reconstruction algorithm for optoacoustic imaging," J. Acoust. Soc. Am., vol. 112, no. 4, pp. 1536-1544, Oct. 2002, doi: 10.1121/1.1501898.

[120] D. Razansky, A. Buehler, and V. Ntziachristos, "Volumetric real-time multispectral optoacoustic tomography of biomarkers," Nat. Protoc., vol. 6, no. 8, pp. 1121-1129, Jul. 2011, doi: 10.1038/nprot.2011.351.

[121] M.-L. Li, H. E. Zhang, K. Maslov, G. Stoica, and L. V. Wang, "Improved in vivo photoacoustic microscopy based on a virtual-detector concept," Opt. Lett., vol. 31, no. 4, pp. 474-476, Feb. 2006, doi: 10.1364/ol.31.000474.

[122] P. Burgholzer, C. Hofer, G. Paltauf, M. Haltmeier, and O. Scherzer, "Thermoacoustic tomography with integrating area and line detectors," IEEE Trans. Ultrason. Ferroelectr. Freq. Control, vol. 52, no. 9, pp. 1577-1583, Sep. 2005, doi: 10.1109/tuffc.2005.1516030.

[123] Y. Xu, L. V. Wang, G. Ambartsoumian, and P. Kuchment, "Reconstructions in limitedview thermoacoustic tomography," Med. Phys., vol. 31, no. 4, pp. 724-733, Apr. 2004, doi: 10.1118/1.1644531.

[124] G. J. Diebold, M. I. Khan, and S. M. Park, "Photoacoustic 'signatures' of particulate matter: optical production of acoustic monopole radiation," Science, vol. 250, no. 4977, pp. 101104, Oct. 1990, doi: 10.1126/science.250.4977.101.

[125] E. M. Strohm, I. Gorelikov, N. Matsuura, and M. C. Kolios, "Acoustic and photoacoustic characterization of micron-sized perfluorocarbon emulsions," J. Biomed. Opt., vol. 17, no. 9, pp. 96016-96011, Sep. 2012, doi: 10.1117/1.JBO.17.9.096016.

[126] R. K. Saha, S. Karmakar, E. Hysi, M. Roy, and M. C. Kolios, "Validity of a theoretical model to examine blood oxygenation dependent optoacoustics," J. Biomed. Opt., vol. 17, no. 5, pp. 0550021-05500210, 2012.

[127] X. L. Dean-Ben and D. Razansky, "On the link between the speckle free nature of optoacoustics and visibility of structures in limited-view tomography," Photoacoustics, vol. 4, no. 4, pp. 133-140, Dec. 2016, doi: 10.1016/j.pacs.2016.10.001.

[128] E. M. Strohm, E. S. L. Berndl, and M. C. Kolios, "Probing Red Blood Cell Morphology Using High-Frequency Photoacoustics,” Biophys. J., vol. 105, no. 1, pp. 59-67, Jul. 2013, doi: 10.1016/j.bpj.2013.05.037.

[129] E. M. Strohm, I. Gorelikov, N. Matsuura, and M. C. Kolios, "Modeling photoacoustic spectral features of micron-sized particles," Phys. Med. Biol., vol. 59, no. 19, pp. 5795-5810, Oct. 2014, doi: 10.1088/0031-9155/59/19/5795.

[130] E. M. Strohm and M. C. Kolios, "Classification of blood cells and tumor cells using labelfree ultrasound and photoacoustics," Cytom. Part J. Int. Soc. Anal. Cytol., vol. 87, no. 8, pp. 741-749, Aug. 2015, doi: 10.1002/cyto.a.22698. 
[131] M. J. Moore, E. M. Strohm, and M. C. Kolios, “Assessment of the Nucleus-to-Cytoplasmic Ratio in MCF-7 Cells Using Ultra-high Frequency Ultrasound and Photoacoustics," Int. J. Thermophys., vol. 37, no. 12, p. 118, Dec. 2016, doi: 10.1007/s10765-016-2129-y.

[132] M. J. Moore et al., "Photoacoustic F-Mode imaging for scale specific contrast in biological systems," Commun. Phys., vol. 2, no. 1, pp. 1-10, Mar. 2019, doi: 10.1038/s42005-019-0131y.

[133] S. Mallidi, K. Watanabe, D. Timerman, D. Schoenfeld, and T. Hasan, "Prediction of Tumor Recurrence and Therapy Monitoring Using Ultrasound-Guided Photoacoustic Imaging," Theranostics, vol. 5, no. 3, pp. 289-301, 2015, doi: 10.7150/thno.10155.

[134] S. E. Bohndiek, L. S. Sasportas, S. Machtaler, J. V. Jokerst, S. Hori, and S. S. Gambhir, "Photoacoustic Tomography Detects Early Vessel Regression and Normalization During Ovarian Tumor Response to the Antiangiogenic Therapy Trebananib," J. Nucl. Med. Off. Publ. Soc. Nucl. Med., vol. 56, no. 12, pp. 1942-1947, Dec. 2015, doi: 10.2967/jnumed.115.160002.

[135] A. Bar-Zion, M. Yin, D. Adam, and F. S. Foster, "Functional Flow Patterns and Static Blood Pooling in Tumors Revealed by Combined Contrast-Enhanced Ultrasound and Photoacoustic Imaging," Cancer Res., vol. 76, no. 15, pp. 4320-4331, Aug. 2016, doi: 10.1158/0008-5472.CAN-16-0376.

[136] L. J. Rich and M. Seshadri, "Photoacoustic monitoring of tumor and normal tissue response to radiation," Sci. Rep., vol. 6, p. 21237, Feb. 2016, doi: 10.1038/srep21237.

[137] Laurie. J. Rich, A. Miller, A. K. Singh, and M. Seshadri, "Photoacoustic Imaging as an Early Biomarker of Radio Therapeutic Efficacy in Head and Neck Cancer," Theranostics, vol. 8, no. 8, pp. 2064-2078, 2018, doi: 10.7150/thno.21708.

[138] S. P. Johnson, O. Ogunlade, M. F. Lythgoe, P. Beard, and R. B. Pedley, "Longitudinal photoacoustic imaging of the pharmacodynamic effect of vascular targeted therapy on tumors," Clin. Cancer Res., Jan. 2019, doi: 10.1158/1078-0432.CCR-19-0360.

[139] J. Zalev and M. C. Kolios, "Detecting abnormal vasculature from photoacoustic signals using wavelet-packet features," in Proc. SPIE, 2011, vol. 7899, pp. 78992M-78992M-15, doi: $10.1117 / 12.873911$.

[140] T. L. Szabo, Diagnostic Ultrasound Imaging: Inside Out, 2 edition. Amsterdam; Boston: Academic Press, 2013.

[141] D. R. Foster, M. Arditi, F. S. Foster, M. S. Patterson, and J. W. Hunt, "Computer Simulations of Speckle in B-Scan Images," Ultrason. Imaging, vol. 5, no. 4, pp. 308-330, Oct. 1983, doi: 10.1177/016173468300500403.

[142] R. S. C. Cobbold, Foundations of Biomedical Ultrasound, 1 edition. Oxford ; New York: Oxford University Press, 2006.

[143] G. Diebold, T. Sun, and M. I. Khan, "Photoacoustic monopole radiation in one, two, and three dimensions," Phys. Rev. Lett., vol. 67, no. 24, pp. 3384-3387, Dec. 1991.

[144] G. Xu et al., "High resolution Physio-chemical Tissue Analysis: Towards Non-invasive In Vivo Biopsy," Sci. Rep., vol. 6, p. 16937, Feb. 2016, doi: 10.1038/srep16937.

[145] T.-H. Bok, E. Hysi, and M. C. Kolios, "In vitro photoacoustic spectroscopy of pulsatile blood flow: probing the interrelationship between red blood cell aggregation and oxygen saturation," J. Biophotonics, Feb. 2018, doi: 10.1002/jbio.201700300.

[146] E. Amidi et al., "Classification of human ovarian cancer using functional, spectral, and imaging features obtained from in vivo photoacoustic imaging," Biomed. Opt. Express, vol. 10, no. 5, pp. 2303-2317, May 2019, doi: 10.1364/BOE.10.002303. 
[147] "S. Prahl, Tabulated data from various sources, http://omlc.ogi.edu/ spectra."

[148] A. Taruttis, S. Morscher, N. C. Burton, D. Razansky, and V. Ntziachristos, "Fast Multispectral Optoacoustic Tomography (MSOT) for Dynamic Imaging of Pharmacokinetics and Biodistribution in Multiple Organs," PLOS ONE, vol. 7, no. 1, p. e30491, Jan. 2012, doi: 10.1371/journal.pone.0030491.

[149] J. Kim et al., "Multispectral ex vivo photoacoustic imaging of cutaneous melanoma for better selection of the excision margin," Br. J. Dermatol., vol. 179, no. 3, pp. 780-782, Sep. 2018, doi: 10.1111/bjd.16677.

[150] J. P. May, E. Hysi, L. A. Wirtzfeld, E. Undzys, S.-D. Li, and M. C. Kolios, "Photoacoustic Imaging of Cancer Treatment Response: Early Detection of Therapeutic Effect from Thermosensitive Liposomes," PLOS ONE, vol. 11, no. 10, p. e0165345, Oct. 2016, doi: 10.1371/journal.pone.0165345.

[151] E. Hysi, L. A. Wirtzfeld, J. P. May, E. Undzys, S.-D. Li, and M. C. Kolios, "Photoacoustic signal characterization of cancer treatment response: Correlation with changes in tumor oxygenation," Photoacoustics, vol. 5, pp. 25-35, Mar. 2017, doi: 10.1016/j.pacs.2017.03.003.

[152] B. Xie et al., "Optoacoustic Detection of Early Therapy-Induced Tumor Cell Death Using a Targeted Imaging Agent," Clin. Cancer Res. Off. J. Am. Assoc. Cancer Res., vol. 23, no. 22, pp. 6893-6903, Nov. 2017, doi: 10.1158/1078-0432.CCR-17-1029.

[153] V. Neuschmelting et al., "WST11 Vascular Targeted Photodynamic Therapy Effect Monitoring by Multispectral Optoacoustic Tomography (MSOT) in Mice," Theranostics, vol. 8, no. 3, pp. 723-734, 2018, doi: 10.7150/thno.20386.

[154] J. C. Dainty, Laser Speckle and Related Phenomena. Springer Science \& Business Media, 2013.

[155] C. B. Burckhardt, "Speckle in ultrasound B-mode scans," IEEE Trans. Sonics Ultrason., vol. 25, no. 1, pp. 1-6, 1978.

[156] J. M. Thijssen and B. J. Oosterveld, "Texture in B-Mode Echograms: A Simulation Study of the Effects of Diffraction and of Scatterer Density on Gray Scale Statistics," in Acoustical Imaging, vol. 14, A. J. Berkhout, J. Ridder, and L. F. van der Wal, Eds. Boston, MA: Springer US, 1985, pp. 481-485.

[157] S. W. Flax, G. H. Glover, and N. J. Pelc, "Textural variations in B-mode ultrasonography: a stochastic model," Ultrason. Imaging, vol. 3, no. 3, pp. 235-257, 1981.

[158] R. F. Wagner, M. F. Insana, and S. W. Smith, "Fundamental correlation lengths of coherent speckle in medical ultrasonic images," IEEE Trans. Ultrason. Ferroelectr. Freq. Control, vol. 35, no. 1, pp. 34-44, 1988, doi: 10.1109/58.4145.

[159] M. J. Moore, S. El-Rass, Y. Xiao, Y. Wang, X.-Y. Wen, and M. C. Kolios, "Simultaneous ultra-high frequency photoacoustic microscopy and photoacoustic radiometry of zebrafish larvae in vivo," Photoacoustics, vol. 12, pp. 14-21, Sep. 2018, doi: 10.1016/j.pacs.2018.08.004.

[160] J. Yao and L. V. Wang, "Sensitivity of photoacoustic microscopy," Photoacoustics, vol. 2, no. 2, pp. 87-101, Jun. 2014, doi: 10.1016/j.pacs.2014.04.002.

[161] Z. Guo, L. Li, and L. V. Wang, "On the speckle-free nature of photoacoustic tomography," Med. Phys., vol. 36, no. 9, pp. 4084-4088, Sep. 2009, doi: 10.1118/1.3187231.

[162] Z. Guo, Z. Xu, and L. V. Wang, "Dependence of photoacoustic speckles on boundary roughness," J. Biomed. Opt., vol. 17, no. 4, p. 046009, Apr. 2012, doi: 10.1117/1.JBO.17.4.046009. 
[163] M. Mehrmohammadi et al., "In vivo Pulsed Magneto-motive Ultrasound Imaging Using High-performance Magnetoactive Contrast Nanoagents," Nanoscale, vol. 5, no. 22, pp. 11179-11186, Nov. 2013, doi: 10.1039/c3nr03669c.

[164] S. Karmakar, E. Hysi, M. C. Kolios, and R. K. Saha, "Realistic photoacoustic image simulations of collections of solid spheres using linear array transducer," presented at the Photons Plus Ultrasound: Imaging and Sensing 2015, Mar. 2015, vol. 9323, p. 932339, doi: 10.1117/12.2080794.

[165] T.-H. Bok, E. Hysi, and M. C. Kolios, "Simultaneous assessment of red blood cell aggregation and oxygen saturation under pulsatile flow using high-frequency photoacoustics," Biomed. Opt. Express, vol. 7, no. 7, pp. 2769-2780, Jun. 2016, doi: 10.1364/BOE.7.002769.

[166] T.-H. Bok, E. Hysi, and M. C. Kolios, "Preliminary Photoacoustic Imaging of the Human Radial Artery for Simultaneous Assessment of Red Blood Cell Aggregation and Oxygen Saturation in Vivo," Ultrason. Symp. IUS 2017 IEEE Int., pp. 1-4, 2017.

[167] J. Mamou et al., "Three-Dimensional High-Frequency Backscatter and Envelope Quantification of Cancerous Human Lymph Nodes," Ultrasound Med. Biol., vol. 37, no. 3, pp. 345-357, Mar. 2011, doi: 10.1016/j.ultrasmedbio.2010.11.020.

[168] R. K. Saha, E. Franceschini, and G. Cloutier, "Assessment of accuracy of the structurefactor-size-estimator method in determining red blood cell aggregate size from ultrasound spectral backscatter coefficient," J. Acoust. Soc. Am., vol. 129, no. 4, pp. 2269-2277, 2011.

[169] A. Sadeghi-Naini et al., "Chemotherapy-Response Monitoring of Breast Cancer Patients Using Quantitative Ultrasound-Based Intra-Tumour Heterogeneities," Sci. Rep., vol. 7, no. 1, p. 10352, Dec. 2017, doi: 10.1038/s41598-017-09678-0.

[170] L. V. Wang, "Tutorial on Photoacoustic Microscopy and Computed Tomography," IEEE J. Sel. Top. Quantum Electron., vol. 14, no. 1, pp. 171-179, Jan. 2008, doi: 10.1109/JSTQE.2007.913398.

[171] S. Umchid, "Directivity pattern measurement of ultrasound transducers," Int. J. Appl. Biomed. Eng. IJABME, vol. 2, pp. 39-43, 2009.

[172] P. M. Shankar, "A general statistical model for ultrasonic backscattering from tissues," IEEE Trans. Ultrason. Ferroelectr. Freq. Control, vol. 47, no. 3, pp. 727-736, 2000.

[173] D. R. Foster, M. Arditi, F. S. Foster, M. S. Patterson, and J. W. Hunt, "Computer Simulations of Speckle in B-Scan Images," Ultrason. Imaging, vol. 5, no. 4, pp. 308-330, Oct. 1983, doi: 10.1177/016173468300500403.

[174] K. A. Wear, R. F. Wagner, M. F. Insana, and T. J. Hall, “Application of autoregressive spectral analysis to cepstral estimation of mean scatterer spacing," IEEE Trans. Ultrason. Ferroelectr. Freq. Control, vol. 40, no. 1, pp. 50-58, Jan. 1993, doi: 10.1109/58.184998.

[175] A. Needles et al., "Development and initial application of a fully integrated photoacoustic micro-ultrasound system," IEEE Trans. Ultrason. Ferroelectr. Freq. Control, vol. 60, no. 5, pp. 888-897, May 2013, doi: 10.1109/TUFFC.2013.2646.

[176] E. Hysi, D. Dopsa, and M. C. Kolios, "Photoacoustic tissue characterization using envelope statistics and ultrasonic spectral parameters," in Proc. SPIE, 2014, vol. 8943, pp. 89432E89432E-9, doi: 10.1117/12.2038624.

[177] J. W. Baish and R. K. Jain, "Fractals and cancer," Cancer Res., vol. 60, no. 14, pp. 36833688, Jul. 2000. 
[178] R. Karshafian, P. N. Burns, and M. R. Henkelman, "Transit time kinetics in ordered and disordered vascular trees," Phys. Med. Biol., vol. 48, no. 19, p. 3225, 2003, doi: 10.1088/0031-9155/48/19/009.

[179] R. K. Jain, J. D. Martin, and T. Stylianopoulos, "The Role of Mechanical Forces in Tumor Growth and Therapy," Annu. Rev. Biomed. Eng., vol. 16, no. 1, pp. 321-346, Jul. 2014, doi: 10.1146/annurev-bioeng-071813-105259.

[180] L. von Baumgarten et al., "Bevacizumab Has Differential and Dose-Dependent Effects on Glioma Blood Vessels and Tumor Cells," Clin. Cancer Res., vol. 17, no. 19, pp. 6192-6205, Oct. 2011, doi: 10.1158/1078-0432.CCR-10-1868.

[181] T. Mathivet et al., "Dynamic stroma reorganization drives blood vessel dysmorphia during glioma growth," EMBO Mol. Med., vol. 9, no. 12, pp. 1629-1645, Dec. 2017, doi: 10.15252/emmm.201607445.

[182] R. C. Molthen, P. M. Shankar, and J. M. Reid, "Characterization of ultrasonic B-scans using non-rayleigh statistics," Ultrasound Med. Biol., vol. 21, no. 2, pp. 161-170, Jan. 1995, doi: 10.1016/S0301-5629(94)00105-7.

[183] P.-H. Tsui et al., "Ultrasonic Nakagami Imaging: A Strategy to Visualize the Scatterer Properties of Benign and Malignant Breast Tumors," Ultrasound Med. Biol., vol. 36, no. 2, pp. 209-217, Feb. 2010, doi: 10.1016/j.ultrasmedbio.2009.10.006.

[184] T. J. Allen and P. C. Beard, "Optimising the detection parameters for deep-tissue photoacoustic imaging," Feb. 2012, p. 82230P, doi: 10.1117/12.908813.

[185] T. Feng et al., "Characterizing cellular morphology by photoacoustic spectrum analysis with an ultra-broadband optical ultrasonic detector," Opt. Express, vol. 24, no. 17, pp. 1985319862, Aug. 2016.

[186] G. Xu, J. B. Fowlkes, C. Tao, X. Liu, and X. Wang, "Photoacoustic spectrum analysis for microstructure characterization in biological tissue: analytical model," Ultrasound Med. Biol., vol. 41, no. 5, pp. 1473-1480, May 2015, doi: 10.1016/j.ultrasmedbio.2015.01.010.

[187] E. Hysi, R. K. Saha, and M. C. Kolios, "Photoacoustic ultrasound spectroscopy for assessing red blood cell aggregation and oxygenation," J. Biomed. Opt., vol. 17, no. 12, pp. 125006-125006, 2012.

[188] S. Wang, C. Tao, Y. Yang, X. Wang, and X. Liu, "Theoretical and experimental study of spectral characteristics of the photoacoustic signal from stochastically distributed particles," IEEE Trans. Ultrason. Ferroelectr. Freq. Control, vol. 62, no. 7, pp. 1245-1255, 2015, doi: 10.1109/TUFFC.2014.006806.

[189] Z. Zhou, L. Sheng, S. Wu, C. Yang, and Y. Zeng, "Ultrasonic evaluation of microwaveinduced thermal lesions based on wavelet analysis of mean scatterer spacing," Ultrasonics, vol. 53, no. 7, pp. 1325-1331, Sep. 2013, doi: 10.1016/j.ultras.2013.03.018.

[190] B. Cox, J. G. Laufer, S. R. Arridge, and P. C. Beard, "Quantitative spectroscopic photoacoustic imaging: a review," J. Biomed. Opt., vol. 17, no. 6, p. 061202, Jun. 2012, doi: 10.1117/1.JBO.17.6.061202.

[191] B. E. Treeby, "Acoustic attenuation compensation in photoacoustic tomography using time-variant filtering," J. Biomed. Opt., vol. 18, no. 3, pp. 036008-036008, 2013.

[192] K. J. Cash, C. Li, J. Xia, L. V. Wang, and H. A. Clark, "Optical Drug Monitoring: Photoacoustic Imaging of Nanosensors to Monitor Therapeutic Lithium in Vivo," ACS Nano, vol. 9, no. 2, pp. 1692-1698, Feb. 2015, doi: 10.1021/nn5064858. 
[193] J. Aguirre et al., "Precision assessment of label-free psoriasis biomarkers with ultrabroadband optoacoustic mesoscopy," Nat. Biomed. Eng., vol. 1, no. 5, p. 0068, May 2017, doi: 10.1038/s41551-017-0068.

[194] J. R. Less, T. C. Skalak, E. M. Sevick, and R. K. Jain, "Microvascular architecture in a mammary carcinoma: branching patterns and vessel dimensions," Cancer Res., vol. 51, no. 1, pp. 265-273, Jan. 1991.

[195] G. P. Luke, J. N. Myers, S. Y. Emelianov, and K. V. Sokolov, "Sentinel lymph node biopsy revisited: ultrasound-guided photoacoustic detection of micrometastases using molecularly targeted plasmonic nanosensors," Cancer Res., vol. 74, no. 19, pp. 5397-5408, Oct. 2014, doi: 10.1158/0008-5472.CAN-14-0796.

[196] J. Ophir et al., "Elastography: ultrasonic estimation and imaging of the elastic properties of tissues," Proc. Inst. Mech. Eng. [H], vol. 213, no. 3, pp. 203-233, 1999, doi: 10.1243/0954411991534933.

[197] B. Zhao, L. H. Schwartz, and S. M. Larson, "Imaging Surrogates of Tumor Response to Therapy: Anatomic and Functional Biomarkers," J. Nucl. Med., vol. 50, no. 2, pp. 239-249, Feb. 2009, doi: 10.2967/jnumed.108.056655.

[198] H. Grüll and S. Langereis, "Hyperthermia-triggered drug delivery from temperaturesensitive liposomes using MRI-guided high intensity focused ultrasound," J. Control. Release Off. J. Control. Release Soc., vol. 161, no. 2, pp. 317-327, Jul. 2012, doi: 10.1016/j.jconrel.2012.04.041.

[199] A. H. Negussie et al., "Formulation and characterisation of magnetic resonance imageable thermally sensitive liposomes for use with magnetic resonance-guided high intensity focused ultrasound," Int. J. Hyperth. Off. J. Eur. Soc. Hyperthermic Oncol. North Am. Hyperth. Group, vol. 27, no. 2, pp. 140-155, 2011, doi: 10.3109/02656736.2010.528140.

[200] M. de Smet, E. Heijman, S. Langereis, N. M. Hijnen, and H. Grüll, "Magnetic resonance imaging of high intensity focused ultrasound mediated drug delivery from temperaturesensitive liposomes: an in vivo proof-of-concept study," J. Control. Release Off. J. Control. Release Soc., vol. 150, no. 1, pp. 102-110, Feb. 2011, doi: 10.1016/j.jconrel.2010.10.036.

[201] T. Ta, E. Bartolak-Suki, E.-J. Park, K. Karrobi, N. J. McDannold, and T. M. Porter, "Localized delivery of doxorubicin in vivo from polymer-modified thermosensitive liposomes with MR-guided focused ultrasound-mediated heating," J. Control. Release Off. J. Control. Release Soc., vol. 194, pp. 71-81, Nov. 2014, doi: 10.1016/j.jconrel.2014.08.013.

[202] J. H. Thrall, "Personalized medicine," Radiology, vol. 231, no. 3, pp. 613-616, Jun. 2004, doi: 10.1148/radiol.2313040323.

[203] B. Kneidl, M. Peller, G. Winter, L. H. Lindner, and M. Hossann, "Thermosensitive liposomal drug delivery systems: state of the art review," Int. J. Nanomedicine, vol. 9, pp. 4387-4398, 2014, doi: 10.2147/IJN.S49297.

[204] J. P. May and S.-D. Li, "Hyperthermia-induced drug targeting," Expert Opin. Drug Deliv., vol. 10, no. 4, pp. 511-527, Apr. 2013, doi: 10.1517/17425247.2013.758631.

[205] C. D. Landon, J.-Y. Park, D. Needham, and M. W. Dewhirst, "Nanoscale Drug Delivery and Hyperthermia: The Materials Design and Preclinical and Clinical Testing of Low Temperature-Sensitive Liposomes Used in Combination with Mild Hyperthermia in the Treatment of Local Cancer," Open Nanomedicine J., vol. 3, pp. 38-64, Jan. 2011, doi: 10.2174/1875933501103010038. 
[206] D. Needham, G. Anyarambhatla, G. Kong, and M. W. Dewhirst, "A new temperaturesensitive liposome for use with mild hyperthermia: characterization and testing in a human tumor xenograft model," Cancer Res., vol. 60, no. 5, pp. 1197-1201, Mar. 2000.

[207] T. Tagami, M. J. Ernsting, and S.-D. Li, "Optimization of a novel and improved thermosensitive liposome formulated with DPPC and a Brij surfactant using a robust in vitro system," J. Controlled Release, vol. 154, no. 3, pp. 290-297, Sep. 2011, doi: 10.1016/j.jconrel.2011.05.020.

[208] T. Tagami, M. J. Ernsting, and S.-D. Li, "Efficient tumor regression by a single and low dose treatment with a novel and enhanced formulation of thermosensitive liposomal doxorubicin," J. Controlled Release, vol. 152, no. 2, pp. 303-309, Jun. 2011, doi: 10.1016/j.jconrel.2011.02.009.

[209] T. Tagami et al., "MRI monitoring of intratumoral drug delivery and prediction of the therapeutic effect with a multifunctional thermosensitive liposome," Biomaterials, vol. 32, no. 27, pp. 6570-6578, Sep. 2011, doi: 10.1016/j.biomaterials.2011.05.029.

[210] T. Tagami, J. P. May, M. J. Ernsting, and S.-D. Li, “A thermosensitive liposome prepared with a $\mathrm{Cu} 2+$ gradient demonstrates improved pharmacokinetics, drug delivery and antitumor efficacy," J. Controlled Release, vol. 161, no. 1, pp. 142-149, Jul. 2012, doi: 10.1016/j.jconrel.2012.03.023.

[211] A. M. Ponce, Z. Vujaskovic, F. Yuan, D. Needham, and M. W. Dewhirst, "Hyperthermia mediated liposomal drug delivery," Int. J. Hyperth. Off. J. Eur. Soc. Hyperthermic Oncol. North Am. Hyperth. Group, vol. 22, no. 3, pp. 205-213, May 2006, doi: 10.1080/02656730600582956.

[212] Q. Chen, S. Tong, M. W. Dewhirst, and F. Yuan, "Targeting tumor microvessels using doxorubicin encapsulated in a novel thermosensitive liposome," Mol. Cancer Ther., vol. 3, no. 10, pp. 1311-1317, 2004.

[213] C. K. Kim, S. Y. Park, B. K. Park, W. Park, and S. J. Huh, "Blood oxygenation leveldependent MR imaging as a predictor of therapeutic response to concurrent chemoradiotherapy in cervical cancer: a preliminary experience," Eur. Radiol., vol. 24, no. 7, pp. 1514-1520, Jul. 2014, doi: 10.1007/s00330-014-3167-0.

[214] J. P. B. O'Connor et al., "Preliminary Study of Oxygen-Enhanced Longitudinal Relaxation in MRI: A Potential Novel Biomarker of Oxygenation Changes in Solid Tumors," Int. J. Radiat. Oncol., vol. 75, no. 4, pp. 1209-1215, Nov. 2009, doi: 10.1016/j.ijrobp.2008.12.040.

[215] I. V. Linnik et al., "Noninvasive tumor hypoxia measurement using magnetic resonance imaging in murine U87 glioma xenografts and in patients with glioblastoma," Magn. Reson. Med., vol. 71, no. 5, pp. 1854-1862, May 2014, doi: 10.1002/mrm.24826.

[216] C. Li and L. V. Wang, "Photoacoustic tomography and sensing in biomedicine," Phys. Med. Biol., vol. 54, no. 19, pp. R59-R97, Oct. 2009, doi: 10.1088/0031-9155/54/19/R01.

[217] S. Y. Emelianov, P.-C. Li, and M. O'Donnell, "Photoacoustics for molecular imaging and therapy," Phys. Today, vol. 62, no. 8, p. 34, 2009.

[218] S. Mallidi, G. P. Luke, and S. Emelianov, "Photoacoustic imaging in cancer detection, diagnosis, and treatment guidance," Trends Biotechnol., vol. 29, no. 5, pp. 213-221, May 2011, doi: 10.1016/j.tibtech.2011.01.006.

[219] L. J. Rich and M. Seshadri, "Photoacoustic imaging of vascular hemodynamics: validation with blood oxygenation level-dependent MR imaging," Radiology, vol. 275, no. 1, pp. 110118, Apr. 2015, doi: 10.1148/radiol.14140654. 
[220] K. E. Wilson, S. V. Bachawal, L. Tian, and J. K. Willmann, "Multiparametric spectroscopic photoacoustic imaging of breast cancer development in a transgenic mouse model," Theranostics, vol. 4, no. 11, pp. 1062-1071, 2014, doi: 10.7150/thno.9922.

[221] M. Gerling et al., "Real-Time Assessment of Tissue Hypoxia In Vivo with Combined Photoacoustics and High-Frequency Ultrasound," Theranostics, vol. 4, no. 6, pp. 604-613, 2014, doi: 10.7150/thno.7996.

[222] R. O. Esenaliev, I. V. Larina, K. V. Larin, D. J. Deyo, M. Motamedi, and D. S. Prough, "Optoacoustic technique for noninvasive monitoring of blood oxygenation: a feasibility study," Appl. Opt., vol. 41, no. 22, pp. 4722-4731, 2002.

[223] X. Wang, X. Xie, G. Ku, L. V. Wang, and G. Stoica, "Noninvasive imaging of hemoglobin concentration and oxygenation in the rat brain using high-resolution photoacoustic tomography," J. Biomed. Opt., vol. 11, no. 2, p. 024015, 2006, doi: 10.1117/1.2192804.

[224] H. Maeda and Y. Matsumura, "EPR effect based drug design and clinical outlook for enhanced cancer chemotherapy," Adv. Drug Deliv. Rev., vol. 63, no. 3, pp. 129-130, Mar. 2011, doi: 10.1016/j.addr.2010.05.001.

[225] S. Park, E. Kim, W. Y. Kim, C. Kang, and J. S. Kim, "Biotin-guided anticancer drug delivery with acidity-triggered drug release," Chem. Commun., vol. 51, no. 45, pp. 93439345, May 2015, doi: 10.1039/C5CC03003J.

[226] T. L. Andresen, D. H. Thompson, and T. Kaasgaard, "Enzyme-triggered nanomedicine: Drug release strategies in cancer therapy (Invited Review)," Mol. Membr. Biol., vol. 27, no. 7, pp. 353-363, Oct. 2010, doi: 10.3109/09687688.2010.515950.

[227] I. M. Hafez and P. R. Cullis, "Tunable pH-sensitive liposomes," Methods Enzymol., vol. 387, pp. 113-134, 2004, doi: 10.1016/S0076-6879(04)87007-1.

[228] R. S. Punglia, M. Morrow, E. P. Winer, and J. R. Harris, "Local Therapy and Survival in Breast Cancer," N. Engl. J. Med., vol. 356, no. 23, pp. 2399-2405, Jun. 2007, doi: 10.1056/NEJMra065241.

[229] C. Furth et al., "Early and late therapy response assessment with [18F]fluorodeoxyglucose positron emission tomography in pediatric Hodgkin's lymphoma: analysis of a prospective multicenter trial," J. Clin. Oncol. Off. J. Am. Soc. Clin. Oncol., vol. 27, no. 26, pp. 43854391, Sep. 2009, doi: 10.1200/JCO.2008.19.7814.

[230] N. G. Mikhaeel, M. Hutchings, P. A. Fields, M. J. O’Doherty, and A. R. Timothy, "FDGPET after two to three cycles of chemotherapy predicts progression-free and overall survival in high-grade non-Hodgkin lymphoma," Ann. Oncol. Off. J. Eur. Soc. Med. Oncol. ESMO, vol. 16, no. 9, pp. 1514-1523, Sep. 2005, doi: 10.1093/annonc/mdi272.

[231] T. Terasawa et al., "Fluorine-18-fluorodeoxyglucose positron emission tomography for interim response assessment of advanced-stage Hodgkin's lymphoma and diffuse large Bcell lymphoma: a systematic review," J. Clin. Oncol. Off. J. Am. Soc. Clin. Oncol., vol. 27, no. 11, pp. 1906-1914, Apr. 2009, doi: 10.1200/JCO.2008.16.0861.

[232] A. Sadeghi-Naini et al., "Early prediction of therapy responses and outcomes in breast cancer patients using quantitative ultrasound spectral texture," Oncotarget, vol. 5, no. 11, pp. 3497-3511, May 2014.

[233] M. R. Bernsen, K. Kooiman, M. Segbers, F. W. B. van Leeuwen, and M. de Jong, "Biomarkers in preclinical cancer imaging," Eur. J. Nucl. Med. Mol. Imaging, vol. 42, no. 4, pp. 579-596, Apr. 2015, doi: 10.1007/s00259-014-2980-7.

[234] J. A. Ludwig and J. N. Weinstein, "Biomarkers in cancer staging, prognosis and treatment selection," Nat. Rev. Cancer, vol. 5, no. 11, pp. 845-856, Nov. 2005, doi: 10.1038/nrc1739. 
[235] A. A. Manzoor et al., "Overcoming limitations in nanoparticle drug delivery: triggered, intravascular release to improve drug penetration into tumors," Cancer Res., vol. 72, no. 21, pp. 5566-5575, Nov. 2012, doi: 10.1158/0008-5472.CAN-12-1683.

[236] T.-J. Yoon and Y.-S. Cho, "Recent advances in photoacoustic endoscopy," World J. Gastrointest. Endosc., vol. 5, no. 11, pp. 534-539, Nov. 2013, doi: 10.4253/wjge.v5.i11.534.

[237] S. R. Beanes, C. Dang, C. Soo, and K. Ting, "Skin repair and scar formation: the central role of TGF-beta," Expert Rev. Mol. Med., vol. 5, no. 8, pp. 1-22, Mar. 2003, doi: 10.1017/S1462399403005817.

[238] T. Tirkes, M. A. Hollar, M. Tann, M. D. Kohli, F. Akisik, and K. Sandrasegaran, "Response criteria in oncologic imaging: review of traditional and new criteria," Radiogr. Rev. Publ. Radiol. Soc. N. Am. Inc, vol. 33, no. 5, pp. 1323-1341, Oct. 2013, doi: 10.1148/rg.335125214.

[239] M. A. Hamburg and F. S. Collins, "The Path to Personalized Medicine," N. Engl. J. Med., vol. 363, no. 4, pp. 301-4, Jul. 2010, doi: http://dx.doi.org/10.1056/NEJMp1006304.

[240] C. P. Adams and V. V. Brantner, "Estimating The Cost Of New Drug Development: Is It Really \$802 Million?," Health Aff. (Millwood), vol. 25, no. 2, pp. 420-428, Mar. 2006, doi: 10.1377/hlthaff.25.2.420.

[241] D. Gonzalez de Castro, P. A. Clarke, B. Al-Lazikani, and P. Workman, "Personalized Cancer Medicine: Molecular Diagnostics, Predictive biomarkers, and Drug Resistance," Clin. Pharmacol. Ther., vol. 93, no. 3, pp. 252-259, Mar. 2013, doi: 10.1038/clpt.2012.237.

[242] H. Tadayyon et al., "Quantitative ultrasound assessment of breast tumor response to chemotherapy using a multi-parameter approach," Oncotarget, Apr. 2016, doi: 10.18632/oncotarget.8862.

[243] L. Sannachi et al., "Non-invasive evaluation of breast cancer response to chemotherapy using quantitative ultrasonic backscatter parameters," Med. Image Anal., vol. 20, no. 1, pp. 224-236, Feb. 2015, doi: 10.1016/j.media.2014.11.009.

[244] R. Choe et al., "Diffuse optical tomography of breast cancer during neoadjuvant chemotherapy: a case study with comparison to MRI," Med. Phys., vol. 32, no. 4, pp. 11281139, Apr. 2005, doi: 10.1118/1.1869612.

[245] L. V. Wang and S. Hu, "Photoacoustic Tomography: In Vivo Imaging from Organelles to Organs," Science, vol. 335, no. 6075, pp. 1458-1462, Mar. 2012, doi: 10.1126/science. 1216210 .

[246] Y. Jin, C. Jia, S.-W. Huang, M. O’Donnell, and X. Gao, "Multifunctional nanoparticles as coupled contrast agents," Nat. Commun., vol. 1, no. 4, pp. 1-8, Jul. 2010, doi: 10.1038/ncomms1042.

[247] M. Heijblom et al., "Photoacoustic image patterns of breast carcinoma and comparisons with Magnetic Resonance Imaging and vascular stained histopathology," Sci. Rep., vol. 5, p. 11778, 2015, doi: 10.1038/srep11778.

[248] C. Cai et al., "In vivo photoacoustic flow cytometry for early malaria diagnosis," Cytom. Part J. Int. Soc. Anal. Cytol., Apr. 2016, doi: 10.1002/cyto.a.22854.

[249] G. Xu et al., "The Functional Pitch of an Organ: Quantification of Tissue Texture with Photoacoustic Spectrum Analysis," Radiology, vol. 271, no. 1, pp. 248-254, Jan. 2014, doi: 10.1148/radiol.13130777.

[250] R. E. Kumon, C. X. Deng, and X. Wang, "Frequency-Domain Analysis of Photoacoustic Imaging Data From Prostate Adenocarcinoma Tumors in a Murine Model," Ultrasound Med. Biol., vol. 37, no. 5, pp. 834-839, May 2011, doi: 10.1016/j.ultrasmedbio.2011.01.012. 
[251] E. Hysi, R. K. Saha, and M. C. Kolios, "On the use of photoacoustics to detect red blood cell aggregation," Biomed. Opt. Express, vol. 3, no. 9, pp. 2326-2338, 2012.

[252] X. Wang et al., "Photoacoustic physio-chemical analysis of liver conditions in animal and human subjects," in Proc. SPIE, 2016, vol. 9708, pp. 97081K-97081K-6, doi: 10.1117/12.2213001.

[253] Q. Li et al., "Photoacoustic spectrum analysis for microstructure characterization using ultra-broad bandwidth optical ultrasonic detector," 2016, vol. 9708, pp. 970812-970812-5, doi: $10.1117 / 12.2211979$.

[254] G. Xu et al., "Photoacoustic and ultrasound dual-modality imaging of human peripheral joints," J. Biomed. Opt., vol. 18, no. 1, pp. 010502-010502, 2013, doi: 10.1117/1.JBO.18.1.010502.

[255] R. K. Saha and M. C. Kolios, "A simulation study on photoacoustic signals from red blood cells," J. Acoust. Soc. Am., vol. 129, no. 5, pp. 2935-2943, May 2011, doi: 10.1121/1.3570946.

[256] R. K. Saha, "A simulation study on the quantitative assessment of tissue microstructure with photoacoustics," IEEE Trans. Ultrason. Ferroelectr. Freq. Control, vol. 62, no. 5, pp. 881-895, May 2015, doi: 10.1109/TUFFC.2015.006993.

[257] M. N. Fadhel, E. Hysi, J. Zalev, and M. C. Kolios, "Photoacoustic simulation of microvessel bleeding: spectral analysis and its implication for monitoring vascular-targeted treatments," in Proc. SPIE, 2016, vol. 9708, pp. 97081B-97081B-10, doi: 10.1117/12.2211774.

[258] P. Shao, D. W. Chapman, R. B. Moore, and R. J. Zemp, "Monitoring photodynamic therapy with photoacoustic microscopy," J. Biomed. Opt., vol. 20, no. 10, p. 106012, Oct. 2015, doi: 10.1117/1.JBO.20.10.106012.

[259] D. Needham and M. W. Dewhirst, "The development and testing of a new temperaturesensitive drug delivery system for the treatment of solid tumors," Adv. Drug Deliv. Rev., vol. 53, no. 3, pp. 285-305, Dec. 2001, doi: 10.1016/S0169-409X(01)00233-2.

[260] K. Nam et al., "Cross-imaging system comparison of backscatter coefficient estimates from a tissue-mimicking material," J. Acoust. Soc. Am., vol. 132, no. 3, pp. 1319-1324, Sep. 2012, doi: 10.1121/1.4742725.

[261] E. Madsen, G. Frank, M. McCormick, M. Deaner, and T. Stiles, "Anechoic sphere phantoms for estimating 3-D resolution of very-high-frequency ultrasound scanners," IEEE Trans. Ultrason. Ferroelectr. Freq. Control, vol. 57, no. 10, pp. 2284-2292, Oct. 2010, doi: 10.1109/TUFFC.2010.1689.

[262] E. L. Madsen, M. E. Deaner, and J. Mehi, "Properties of phantom tissuelike polymethylpentene in the frequency range 20-70 MHZ," Ultrasound Med. Biol., vol. 37, no. 8, pp. 1327-1339, Aug. 2011, doi: 10.1016/j.ultrasmedbio.2011.05.023.

[263] L. Wang, K. Maslov, W. Xing, A. Garcia-Uribe, and L. V. Wang, "Video-rate functional photoacoustic microscopy at depths," J. Biomed. Opt., vol. 17, no. 10, pp. 1060071-1060075, 2012, doi: 10.1117/1.JBO.17.10.106007.

[264] E. A. Eisenhauer et al., "New response evaluation criteria in solid tumours: revised RECIST guideline (version 1.1)," Eur. J. Cancer Oxf. Engl. 1990, vol. 45, no. 2, pp. 228247, Jan. 2009, doi: 10.1016/j.ejca.2008.10.026.

[265] R. K. Jain, "Molecular regulation of vessel maturation," Nat. Med., vol. 9, no. 6, pp. 685693, Jun. 2003, doi: 10.1038/nm0603-685. 
[266] Q. Chen, A. Krol, A. Wright, D. Needham, M. W. Dewhirst, and F. Yuan, "Tumor microvascular permeability is a key determinant for antivascular effects of doxorubicin encapsulated in a temperature sensitive liposome," Int. J. Hyperthermia, vol. 24, no. 6, pp. 475-482, Jan. 2008, doi: 10.1080/02656730701854767.

[267] G. Xu, I. A. Dar, C. Tao, X. Liu, C. X. Deng, and X. Wang, "Photoacoustic spectrum analysis for microstructure characterization in biological tissue: A feasibility study," Appl. Phys. Lett., vol. 101, no. 22, p. 221102, Nov. 2012, doi: 10.1063/1.4768703.

[268] M. Gerling et al., "Real-time assessment of tissue hypoxia in vivo with combined photoacoustics and high-frequency ultrasound," Theranostics, vol. 4, no. 6, pp. 604-613, 2014, doi: 10.7150/thno.7996.

[269] L. Li et al., "Mild hyperthermia triggered doxorubicin release from optimized stealth thermosensitive liposomes improves intratumoral drug delivery and efficacy," J. Control. Release Off. J. Control. Release Soc., vol. 168, no. 2, pp. 142-150, Jun. 2013, doi: 10.1016/j.jconrel.2013.03.011.

[270] M. N. Fadhel, E. Hysi, J. Zalev, and M. C. Kolios, "Photoacoustic simulation of microvessel bleeding: spectral analysis and its implication for monitoring vascular-targeted treatments," Mar. 2016, p. 97081B, doi: 10.1117/12.2211774.

[271] T. N. Erpelding et al., "Sentinel Lymph Nodes in the Rat: Noninvasive Photoacoustic and US Imaging with a Clinical US System 1,” Radiology, vol. 256, no. 1, pp. 102-110, 2010.

[272] R. M. Vlad, N. M. Alajez, A. Giles, M. C. Kolios, and G. J. Czarnota, "Quantitative ultrasound characterization of cancer radiotherapy effects in vitro," Int. J. Radiat. Oncol. Biol. Phys., vol. 72, no. 4, pp. 1236-1243, Nov. 2008, doi: 10.1016/j.ijrobp.2008.07.027.

[273] M. L. Oelze, W. D. O’Brien, J. P. Blue, and J. F. Zachary, "Differentiation and characterization of rat mammary fibroadenomas and 4T1 mouse carcinomas using quantitative ultrasound imaging," IEEE Trans. Med. Imaging, vol. 23, no. 6, pp. 764-771, Jun. 2004.

[274] M. B. Amin et al., "The Eighth Edition AJCC Cancer Staging Manual: Continuing to build a bridge from a population-based to a more "personalized' approach to cancer staging," $C A$. Cancer J. Clin., vol. 67, no. 2, pp. 93-99, Mar. 2017, doi: 10.3322/caac.21388.

[275] D. Cross and J. K. Burmester, "Gene therapy for cancer treatment: past, present and future," Clin. Med. Res., vol. 4, no. 3, pp. 218-227, Sep. 2006, doi: 10.3121/cmr.4.3.218.

[276] E. I. Dumbrava and F. Meric-Bernstam, "Personalized cancer therapy-leveraging a knowledge base for clinical decision-making," Cold Spring Harb. Mol. Case Stud., vol. 4, no. 2, Apr. 2018, doi: 10.1101/mcs.a001578.

[277] M. Verma, "Personalized Medicine and Cancer," J. Pers. Med., vol. 2, no. 1, pp. 1-14, Jan. 2012, doi: 10.3390/jpm2010001.

[278] C. L. Vogel et al., "Efficacy and safety of trastuzumab as a single agent in first-line treatment of HER2-overexpressing metastatic breast cancer," J. Clin. Oncol. Off. J. Am. Soc. Clin. Oncol., vol. 20, no. 3, pp. 719-726, Feb. 2002, doi: 10.1200/JCO.2002.20.3.719.

[279] T. Vu, D. Razansky, and J. Yao, "Listening to tissues with new light: recent technological advances in photoacoustic imaging," J. Opt., vol. 21, no. 10, p. 103001, Sep. 2019, doi: 10.1088/2040-8986/ab3b1a.

[280] S. Manohar and M. Dantuma, "Current and future trends in photoacoustic breast imaging," Photoacoustics, vol. 16, p. 100134, Dec. 2019, doi: 10.1016/j.pacs.2019.04.004. 
[281] E. Hysi, M. N. Fadhel, M. J. Moore, J. Zalev, E. M. Strohm, and M. C. Kolios, "Insights into photoacoustic speckle and applications in tumor characterization," Photoacoustics, vol. 14, pp. 37-48, Jun. 2019, doi: 10.1016/j.pacs.2019.02.002.

[282] C. Bing et al., "Characterization of different bubble formulations for blood-brain barrier opening using a focused ultrasound system with acoustic feedback control," Sci. Rep., vol. 8, no. 1, pp. 1-12, May 2018, doi: 10.1038/s41598-018-26330-7.

[283] P. Nittayacharn, H.-X. Yuan, C. Hernandez, P. Bielecki, H. Zhou, and A. A. Exner, "Enhancing Tumor Drug Distribution With Ultrasound-Triggered Nanobubbles," J. Pharm. Sci., vol. 108, no. 9, pp. 3091-3098, Sep. 2019, doi: 10.1016/j.xphs.2019.05.004.

[284] C. Hernandez et al., "Sink or float? Characterization of shell-stabilized bulk nanobubbles using a resonant mass measurement technique," Nanoscale, vol. 11, no. 3, pp. 851-855, Jan. 2019, doi: 10.1039/c8nr08763f.

[285] A. de Leon et al., "Contrast enhanced ultrasound imaging by nature-inspired ultrastable echogenic nanobubbles," Nanoscale, vol. 11, no. 33, pp. 15647-15658, Sep. 2019, doi: 10.1039/c9nr04828f.

[286] X. Liang, Y. Xu, C. Gao, Y. Zhou, N. Zhang, and Z. Dai, "Ultrasound contrast agent microbubbles with ultrahigh loading capacity of camptothecin and floxuridine for enhancing tumor accumulation and combined chemotherapeutic efficacy," NPG Asia Mater., vol. 10, no. 8, pp. 761-774, Aug. 2018, doi: 10.1038/s41427-018-0066-x.

[287] C. Holland, "Ultrasound Contrast Agents Accelerate Sonothrombolysis," Ultrasound Med. Biol., vol. 41, no. 4, p. S94, Apr. 2015, doi: 10.1016/j.ultrasmedbio.2014.12.377.

[288] K. Hynynen, N. McDannold, N. Vykhodtseva, and F. A. Jolesz, "Noninvasive MR imaging-guided focal opening of the blood-brain barrier in rabbits," Radiology, vol. 220, no. 3, pp. 640-646, Sep. 2001, doi: 10.1148/radiol.2202001804.

[289] A. J. Sojahrood, L. Nieves, C. Hernandez, A. Exner, and M. C. Kolios, "Theoretical and experimental investigation of the nonlinear dynamics of nanobubbles excited at clinically relevant ultrasound frequencies and pressures: The role of lipid shell buckling," in 2017 IEEE International Ultrasonics Symposium (IUS), Sep. 2017, pp. 1-1, doi: 10.1109/ULTSYM.2017.8091934.

[290] Y. Gao et al., "Ultrasound molecular imaging of ovarian cancer with CA-125 targeted nanobubble contrast agents," Nanomedicine Nanotechnol. Biol. Med., vol. 13, no. 7, pp. 2159-2168, Oct. 2017, doi: 10.1016/j.nano.2017.06.001.

[291] J. Lee, R. Karshafian, N. Papanicolau, A. Giles, M. C. Kolios, and G. J. Czarnota, "Quantitative ultrasound for the monitoring of novel microbubble and ultrasound radiosensitization," Ultrasound Med. Biol., vol. 38, no. 7, pp. 1212-1221, Jul. 2012, doi: 10.1016/j.ultrasmedbio.2012.01.028.

[292] G. J. Czarnota et al., "Tumor radiation response enhancement by acoustical stimulation of the vasculature," Proc. Natl. Acad. Sci. U. S. A., vol. 109, no. 30, pp. E2033-E2041, Jul. 2012, doi: 10.1073/pnas.1200053109.

[293] A. A. Al-Mahrouki, S. Iradji, W. T. Tran, and G. J. Czarnota, "Cellular characterization of ultrasound-stimulated microbubble radiation enhancement in a prostate cancer xenograft model," Dis. Model. Mech., vol. 7, no. 3, pp. 363-372, Mar. 2014, doi: 10.1242/dmm.012922.

[294] H. Wu, E. C. Abenojar, R. Perera, A. C. De Leon, T. An, and A. A. Exner, "Time-intensitycurve Analysis and Tumor Extravasation of Nanobubble Ultrasound Contrast Agents," Ultrasound Med. Biol., vol. 45, no. 9, pp. 2502-2514, Sep. 2019, doi: 10.1016/j.ultrasmedbio.2019.05.025. 
[295] R. Karshafian, P. D. Bevan, R. Williams, S. Samac, and P. N. Burns, "Sonoporation by ultrasound-activated microbubble contrast agents: effect of acoustic exposure parameters on cell membrane permeability and cell viability," Ultrasound Med. Biol., vol. 35, no. 5, pp. 847-860, May 2009, doi: 10.1016/j.ultrasmedbio.2008.10.013.

[296] L. Wang, K. Maslov, W. Xing, A. Garcia-Uribe, and L. V. Wang, "Video-rate functional photoacoustic microscopy at depths," J. Biomed. Opt., vol. 17, no. 10, pp. 1060071-1060075, 2012, doi: 10.1117/1.JBO.17.10.106007.

[297] G. Xu et al., "The Functional Pitch of an Organ: Quantification of Tissue Texture with Photoacoustic Spectrum Analysis," Radiology, vol. 271, no. 1, pp. 248-254, Apr. 2014, doi: 10.1148/radiol.13130777.

[298] G. A. F. Seber and A. J. Lee, Linear Regression Analysis. John Wiley \& Sons, 2012.

[299] O. V. Solesvik, E. K. Rofstad, and T. Brustad, "Vascular changes in a human malignant melanoma xenograft following single-dose irradiation," Radiat. Res., vol. 98, no. 1, pp. 115128, Apr. 1984.

[300] M. H. M. Janssen et al., "Tumor perfusion increases during hypofractionated short-course radiotherapy in rectal cancer: sequential perfusion-CT findings," Radiother. Oncol. J. Eur. Soc. Ther. Radiol. Oncol., vol. 94, no. 2, pp. 156-160, Feb. 2010, doi: 10.1016/j.radonc.2009.12.013.

[301] A. G. Orlova et al., "Diffuse optical spectroscopy assessment of rodent tumor model oxygen state after single-dose irradiation," Biomed. Phys. Eng. Express, vol. 5, no. 3, p. 035010, Mar. 2019, doi: 10.1088/2057-1976/ab0b19.

[302] G. Diot et al., "Multispectral Optoacoustic Tomography (MSOT) of Human Breast Cancer," Clin. Cancer Res. Off. J. Am. Assoc. Cancer Res., vol. 23, no. 22, pp. 6912-6922, Nov. 2017, doi: 10.1158/1078-0432.CCR-16-3200.

[303] B. Cheng, C. Bing, Y. Xi, B. Shah, A. A. Exner, and R. Chopra, "Influence of Nanobubble Concentration on Blood-Brain Barrier Opening Using Focused Ultrasound Under Real-Time Acoustic Feedback Control," Ultrasound Med. Biol., vol. 45, no. 8, pp. 2174-2187, Aug. 2019, doi: 10.1016/j.ultrasmedbio.2019.03.016.

[304] E. C. Abenojar et al., "Theoretical and Experimental Gas Volume Quantification of Microand Nanobubble Ultrasound Contrast Agents," Pharmaceutics, vol. 12, no. 3, Mar. 2020, doi: 10.3390/pharmaceutics12030208.

[305] G. Yang et al., "Co-registered photoacoustic and ultrasound imaging of human colorectal cancer," J. Biomed. Opt., vol. 24, no. 12, pp. 1-13, Nov. 2019, doi: 10.1117/1.JBO.24.12.121913.

[306] M. N. Fadhel, E. Hysi, J. Zalev, and M. C. Kolios, "Photoacoustic simulations of microvascular bleeding: spectral analysis and its application for monitoring vascular-targeted treatments," J. Biomed. Opt., vol. 24, no. 11, pp. 1-8, 2019, doi: 10.1117/1.JBO.24.11.116001.

[307] X. Leng et al., "Feasibility of co-registered ultrasound and acoustic-resolution photoacoustic imaging of human colorectal cancer," Biomed. Opt. Express, vol. 9, no. 11, pp. 5159-5172, Nov. 2018, doi: 10.1364/BOE.9.005159.

[308] B. T. Cox, S. R. Arridge, and P. C. Beard, "Estimating chromophore distributions from multiwavelength photoacoustic images," J. Opt. Soc. Am. A Opt. Image Sci. Vis., vol. 26, no. 2, pp. 443-455, Feb. 2009, doi: 10.1364/josaa.26.000443. 
[309] R. Hochuli, L. An, P. C. Beard, and B. T. Cox, "Estimating blood oxygenation from photoacoustic images: can a simple linear spectroscopic inversion ever work?," J. Biomed. Opt., vol. 24, no. 12, pp. 1-13, 2019, doi: 10.1117/1.JBO.24.12.121914.

[310] L. An, T. Saratoon, M. Fonseca, R. Ellwood, and B. Cox, "Statistical independence in nonlinear model-based inversion for quantitative photoacoustic tomography," Biomed. Opt. Express, vol. 8, no. 11, pp. 5297-5310, Nov. 2017, doi: 10.1364/BOE.8.005297.

[311] B. T. Cox, J. G. Laufer, and P. C. Beard, "Quantitative Photoacoustic Image Reconstruction using Fluence Dependent Chromophores," Biomed. Opt. Express, vol. 1, no. 1, pp. 201-208, Jul. 2010, doi: 10.1364/BOE.1.000201.

[312] S. Tzoumas et al., "Eigenspectra optoacoustic tomography achieves quantitative blood oxygenation imaging deep in tissues," Nat. Commun., vol. 7, p. 12121, 30 2016, doi: $10.1038 /$ ncomms 12121 .

[313] G. Paltauf, P. R. Torke, and R. Nuster, "Modeling photoacoustic imaging with a scanning focused detector using Monte Carlo simulation of energy deposition," J. Biomed. Opt., vol. 23, no. 12, pp. 1-11, 2018, doi: 10.1117/1.JBO.23.12.121607.

[314] R. Lencioni and D. Cioni, "RFA plus lyso-thermosensitive liposomal doxorubicin: in search of the optimal approach to cure intermediate-size hepatocellular carcinoma," Hepatic Oncol., vol. 3, no. 3, pp. 193-200, Aug. 2016, doi: 10.2217/hep-2016-0005.

[315] H. C. Kim, A. Al-Mahrouki, A. Gorjizadeh, A. Sadeghi-Naini, R. Karshafian, and G. J. Czarnota, "Quantitative Ultrasound Characterization of Tumor Cell Death: UltrasoundStimulated Microbubbles for Radiation Enhancement," PLOS ONE, vol. 9, no. 7, Jul. 2014, doi: 10.1371/journal.pone.0102343.

[316] S. Dutta and P. Sengupta, "Men and mice: Relating their ages," Life Sci., vol. 152, pp. 244 248, May 2016, doi: 10.1016/j.lfs.2015.10.025.

[317] M. N. Bouchlaka et al., "Aging predisposes to acute inflammatory induced pathology after tumor immunotherapy," J. Exp. Med., vol. 210, no. 11, pp. 2223-2237, Oct. 2013, doi: 10.1084/jem.20131219.

[318] C. H. Kugel et al., "Age Correlates with Response to Anti-PD1, Reflecting Age-Related Differences in Intratumoral Effector and Regulatory T-Cell Populations," Clin. Cancer Res. Off. J. Am. Assoc. Cancer Res., vol. 24, no. 21, pp. 5347-5356, 01 2018, doi: 10.1158/10780432.CCR-18-1116.

[319] R. K. Jain, "Determinants of tumor blood flow: a review," Cancer Res., vol. 48, no. 10, pp. 2641-2658, 1988.

[320] M. A. Pozniak and P. L. Allan, Clinical Doppler Ultrasound: Expert Consult, 3 edition. Elsevier Canada, 2013.

[321] J. Brunker and P. Beard, "Acoustic resolution photoacoustic Doppler velocimetry in bloodmimicking fluids," Sci. Rep., vol. 6, p. 20902, 2016, doi: 10.1038/srep20902.

[322] P. J. van den Berg, K. Daoudi, and W. Steenbergen, "Review of photoacoustic flow imaging: its current state and its promises," Photoacoustics, vol. 3, no. 3, pp. 89-99, Sep. 2015, doi: 10.1016/j.pacs.2015.08.001.

[323] J. Brunker and P. Beard, "Pulsed photoacoustic Doppler flowmetry using time-domain cross-correlation: accuracy, resolution and scalability," J. Acoust. Soc. Am., vol. 132, no. 3, pp. 1780-1791, Sep. 2012, doi: 10.1121/1.4739458.

[324] J. Brunker and P. Beard, "Pulsed photoacoustic Doppler flow measurements in bloodmimicking phantoms," Feb. 2011, pp. 78991K-78991K-10, doi: 10.1117/12.874469. 
[325] Y. Jiang and R. Zemp, "Estimation of cerebral metabolic rate of oxygen consumption using combined multiwavelength photoacoustic microscopy and Doppler microultrasound," $J$. Biomed. Opt., vol. 23, no. 1, p. 016009, Jan. 2018, doi: 10.1117/1.JBO.23.1.016009.

[326] J. Yao, K. I. Maslov, Y. Zhang, Y. Xia, and L. V. Wang, "Label-free oxygen-metabolic photoacoustic microscopy in vivo," J. Biomed. Opt., vol. 16, no. 7, Jul. 2011, doi: 10.1117/1.3594786.

[327] C. Walker, E. Mojares, and A. del Río Hernández, "Role of Extracellular Matrix in Development and Cancer Progression," Int. J. Mol. Sci., vol. 19, no. 10, Oct. 2018, doi: 10.3390/ijms 19103028 .

[328] V. P. Chauhan et al., "Angiotensin inhibition enhances drug delivery and potentiates chemotherapy by decompressing tumour blood vessels," Nat. Commun., vol. 4, Oct. 2013, doi: $10.1038 /$ ncomms 3516 .

[329] B. Diop-Frimpong, V. P. Chauhan, S. Krane, Y. Boucher, and R. K. Jain, "Losartan inhibits collagen I synthesis and improves the distribution and efficacy of nanotherapeutics in tumors," Proc. Natl. Acad. Sci. U. S. A., vol. 108, no. 7, pp. 2909-2914, Feb. 2011, doi: 10.1073/pnas.1018892108.

[330] J. D. Martin, D. Fukumura, D. G. Duda, Y. Boucher, and R. K. Jain, "Reengineering the Tumor Microenvironment to Alleviate Hypoxia and Overcome Cancer Heterogeneity," Cold Spring Harb. Perspect. Med., vol. 6, no. 12, Dec. 2016, doi: 10.1101/cshperspect.a027094.

[331] S. Xu et al., "The role of collagen in cancer: from bench to bedside," J. Transl. Med., vol. 17, no. 1, p. 309, Sep. 2019, doi: 10.1186/s12967-019-2058-1.

[332] P. J. van den Berg, R. Bansal, K. Daoudi, W. Steenbergen, and J. Prakash, "Preclinical detection of liver fibrosis using dual-modality photoacoustic/ultrasound system," Biomed. Opt. Express, vol. 7, no. 12, pp. 5081-5091, Nov. 2016, doi: 10.1364/BOE.7.005081.

[333] Y. Zhu et al., "Identifying intestinal fibrosis and inflammation by spectroscopic photoacoustic imaging: an animal study in vivo," Biomed. Opt. Express, vol. 9, no. 4, pp. 1590-1600, Mar. 2018, doi: 10.1364/BOE.9.001590.

[334] A. P. Regensburger et al., "Detection of collagens by multispectral optoacoustic tomography as an imaging biomarker for Duchenne muscular dystrophy," Nat. Med., vol. 25, pp. 1905-1915, Dec. 2019, doi: 10.1038/s41591-019-0669-y.

[335] L. Sannachi et al., "Response monitoring of breast cancer patients receiving neoadjuvant chemotherapy using quantitative ultrasound, texture, and molecular features," PLoS ONE, vol. 13, no. 1, Jan. 2018, doi: 10.1371/journal.pone.0189634.

[336] H. Tadayyon et al., "A priori Prediction of Neoadjuvant Chemotherapy Response and Survival in Breast Cancer Patients using Quantitative Ultrasound," Sci. Rep., vol. 7, no. 1, pp. 1-11, Apr. 2017, doi: 10.1038/srep45733.

[337] H. Tadayyon et al., "A priori prediction of breast tumour response to chemotherapy using quantitative ultrasound imaging and artificial neural networks," Oncotarget, vol. 10, no. 39, pp. 3910-3923, Jun. 2019, doi: 10.18632/oncotarget.26996. 